DOE/ID-10511

May 1995

RE

ОСТ 2 U 1995

OSTI

\title{
Validation of the
}

Transportation Computer Codes HIGHWAY, INTERLINE, RADTRAN 4, and RISKIND

\section{Idaho National Engineering Laboratory}

U.S. Department of Energy $\cdot /$ daho Operations Office

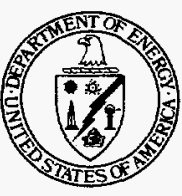

MASTER 


\section{DISCLAIMER}

Portions of this document may be illegible in electronic image products. Images are produced from the best available original document. 


\section{Validation of the Transportation Computer Codes HIGHWAY, INTERLINE, RADTRAN 4, and RISKIND}

Steven J. Maheras

Howard K. Pippen

May 1995

Science Applications International Corporation

545 Shoup Avenue Idaho Falls, Idaho 83402

Prepared for the U.S. Department of Energy

EIS Project Office

Under DOE Idaho Operations Office

Contract No. DE-AC07-92ID-13208

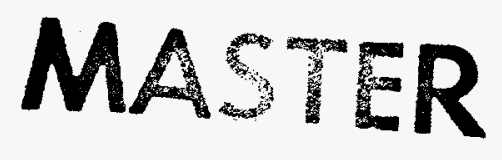




\section{ABSTRACT}

The computer codes HIGHWAY, INTERLINE, RADTRAN 4, and RISKIND were used to estimate radiation doses from the transportation of radioactive material in the Department of Energy Programmatic Spent Nuclear Fuel Management and Idaho National Engineering Laboratory Environmental Restoration and Waste Management Programs Environmental Impact Statement.

HIGHWAY and INTERLINE were used to estimate transportation routes for truck and rail shipments, respectively. RADTRAN 4 was used to estimate collective doses from incident-free transportation and the risk (probability $\times$ consequence) from transportation accidents. RISKNND was used to estimate incident-free radiation doses for maximally exposed individuals and the consequences from reasonably foreseeable transportation accidents. The purpose of this analysis is to validate the estimates made by these computer codes; critiques of the conceptual models used in RADTRAN 4 are also discussed. Validation is defined as "the test and evaluation of the completed software to ensure compliance with software requirements." In this analysis, validation means that the differences between the estimates generated by these codes and independent observations are small (i.e., within the acceptance criterion established for the validation analysis). In some cases, the independent observations used in the validation were measurements; in other cases, the independent observations used in the validation analysis were generated using hand calculations. The results of the validation analyses performed for HIGHWAY, INTERLINE, RADTRAN 4, and RISKIND show that the differences between the estimates generated using the computer codes and independent observations were small. Based on the acceptance criterion established for the validation analyses, the codes yielded acceptable results; in all cases the estimates met the requirements for successful validation.

\section{DISCLAIMER}

\footnotetext{
This report was prepared as an account of work sponsored by an agency of the United States Government. Neither the United States Government nor any agency thereof, nor any of their employees, makes any warranty, express or implied, or assumes any legal liability or responsibility for the accuracy, completeness, or usefulness of any information, apparatus, product, or process disclosed, or represents that its use would not infringe privately owned rights. Reference herein to any specific commercial product, process, or service by trade name, trademark, manufacturer, or otherwise does not necessarily constitute or imply its endorsement, recommendation, or favoring by the United States Government or any agency thereof. The views United States Government or any agency thereof.
} 



\section{CONTENTS}

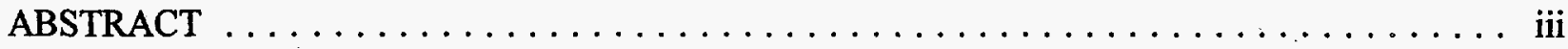

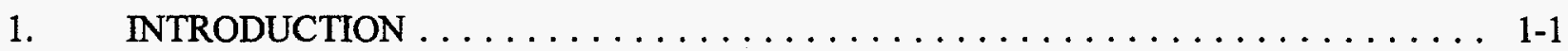

2. VALIDATION OF THE HIGHWAY AND INTERLINE RADIOACTIVE MATERIALS TRANSPORTATION ROUTING COMPUTER CODES $\ldots \ldots \ldots \ldots$ 2-1

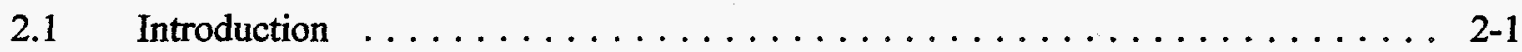

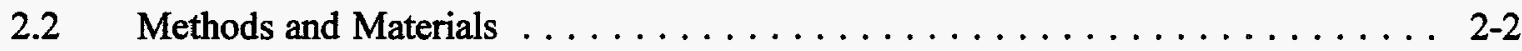

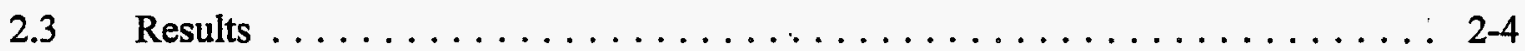

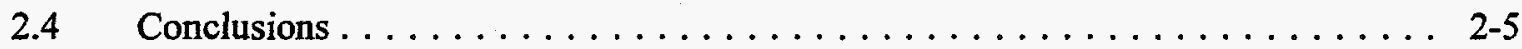

3. VALIDATION OF THE INCIDENT-FREE UNIT RISK FACTORS CALCULATED USING THE RADTRAN 4 COMPUTER CODE $\ldots \ldots \ldots \ldots \ldots \ldots \ldots \ldots \ldots .1$

$3.1 \quad$ Validation Analysis $\ldots \ldots \ldots \ldots \ldots \ldots \ldots \ldots \ldots \ldots \ldots \ldots \ldots$

Results of Validation Analysis $\ldots \ldots \ldots \ldots \ldots \ldots \ldots \ldots \ldots \ldots \ldots \ldots$

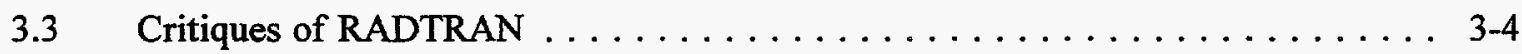

3.4 Detailed Hand Calculations for Incident-Free Truck Unit Risk Factors . . . . . 3-7

3.4.1 List of Symbols - Truck $\ldots \ldots \ldots \ldots \ldots \ldots \ldots \ldots \ldots \ldots$ 3-7

3.4 .2 Crew Dose - Truck . . . . . . . . . . . . . . . . . 3-9

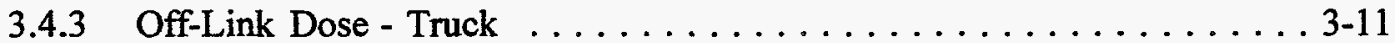

3.4.4 On-Link Dose - Truck . . . . . . . . . . . . . . . . . . . . 3-14

3.4.5 Dose at Stops - Truck . . . . . . . . . . . . . . . . 3-26

3.5 Detailed Hand Calculations for Incident-Free Rail Unit Risk Factors $\ldots \ldots \ldots$ 3-28

3.5.1 List of Symbols - Truck . . . . . . . . . . . . . . . 3-28

3.5.2 Linear Rural, Suburban, and Urban Crew Dose - Rail . . . . . . . . . . 3-30

3.5.3 Nonlinear Rural, Suburban, and Urban Crew Dose - Rail . . . . . . . . . . 3-30

3.5.4 Off-Link Dose - Rail . . . . . . . . . . . . . . . . . . . . 3-31

3.5.5 On-Link Dose - Rail . . . . . . . . . . . . . . . . 3-33

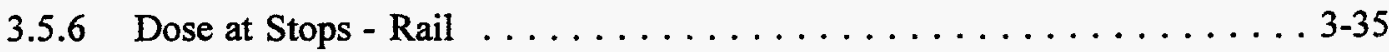

4. VALIDATION OF THE ACCIDENT RISKS CALCULATED USING THE

RADTRAN 4 COMPUTER CODE $\ldots \ldots \ldots \ldots \ldots \ldots \ldots \ldots \ldots \ldots \ldots \ldots \ldots$

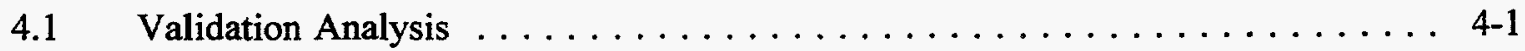

4.2 Results of Validation Analysis $\ldots \ldots \ldots \ldots \ldots \ldots \ldots \ldots \ldots \ldots$ 
$4.3 \quad$ Critiques of RADTRAN $\ldots \ldots \ldots \ldots \ldots \ldots \ldots \ldots \ldots \ldots \ldots$

4.4 Detailed Hand Calculations for Accident Risks $\ldots \ldots \ldots \ldots \ldots \ldots \ldots \ldots$. . . . .

4.4.1 List of Symbols $\ldots \ldots \ldots \ldots \ldots \ldots \ldots \ldots \ldots \ldots \ldots \ldots$

4.4 .2 Groundshine Pathway . . . . . . . . . . . . . . . 4-9

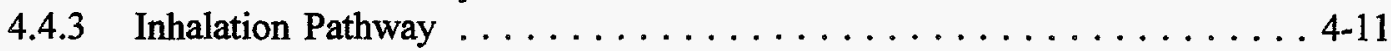

4.4 .4 Resuspension Pathway . . . . . . . . . . . . . . . . 4-12

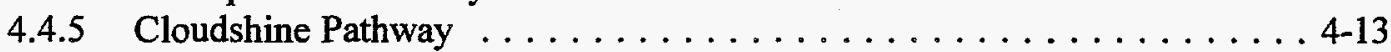

4.4 .6 Ingestion Pathway $\ldots \ldots \ldots \ldots \ldots \ldots \ldots \ldots \ldots \ldots$ 4-14

5. VALIDATION OF THE INCIDENT-FREE RADIATION DOSES CALCULATED USING THE RISKIND COMPUTER CODE . . . . . . . . . . . . . . 5-1

$5.1 \quad$ Validation Analysis $\ldots \ldots \ldots \ldots \ldots \ldots \ldots \ldots \ldots \ldots \ldots \ldots \ldots$

5.2 Results of Validation Analysis $\ldots \ldots \ldots \ldots \ldots \ldots \ldots \ldots \ldots \ldots \ldots$

6. VALIDATION OF THE ACCIDENT RADIATION DOSES CALCULATED USING THE RISKIND COMPUTER CODE . . . . . . . . . . . . . . . 6-1

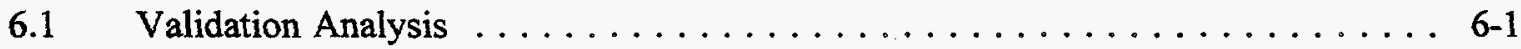

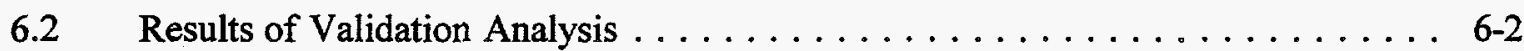

6.3 Detailed Hand Calculations for the Transportation Accident Scenario . . . . . . . 6-3

6.3 .1 List of Symbols ... . . . . . . . . . . . . . . . . 6 6-3

6.3.2 Atmospheric Concentration at Receptor ................ 6. 6.5

6.3.3 Short-Term Radiation Doses ...................... 6-6

6.3.4 Long-Term Radiation Doses . . . . . . . . . . . . . . . . . 6-8

7. REFERENCES .......................... $7-1$

APPENDIX A-RADTRAN 4 OUTPUT FOR INCIDENT-FREE UNIT RISK FACTORS . . . . A-1

APPENDIX B-RADTRAN 4 OUTPUT FOR ACCIDENT RISKS $\ldots \ldots \ldots \ldots \ldots \ldots$. . . B

APPENDIX C-RISKIND OUTPUT FOR INCIDENT-FREE MAXIMALLY EXPOSED

INDIVIDUAL SCENARIOS $\ldots \ldots \ldots \ldots \ldots \ldots \ldots \ldots \ldots \ldots \ldots \ldots$

APPENDIX D-RISKIND OUTPUT FOR TRANSPORTATION ACCIDENT SCENARIO . . . D-1

\section{TABLES}

2-1. Centralization at the Hanford Site cumulative shipment-miles for truck shipments . . . . 2-6

2-2. Centralization at the Savannah River Site cumulative shipment-miles for truck shipments . . . . . . . . . . . . . . . . . . . . . . . . . . 2-7 
2-3. Centralization at the Idaho National Engineering Laboratory cumulative shipment-miles

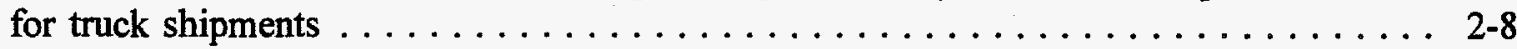

2-4. Centralization at the Oak Ridge Reservation cumulative shipment-miles for truck shipments . . . . . . . . . . . . . . . . . . .

2-5. Centralization at the Nevada Test Site cumulative shipment-miles for truck shipments . . 2-10

2-6. Centralization at the Hanford Site cumulative shipment-miles for train shipments $\ldots \ldots$ 2-11

2-7. Centralization at the Savannah River Site cumulative shipment-miles for train

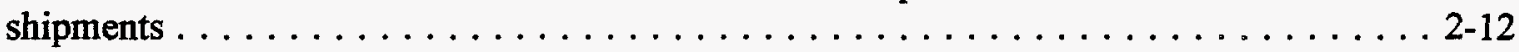

2-8. Centralization at the Idaho National Engineering Laboratory cumulative shipment-miles

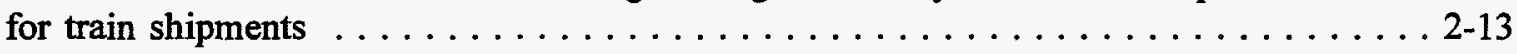

2-9. Centralization at the Oak Ridge Reservation cumulative shipment-miles for train shipments . . . . . . . . . . . . . . . . . . . . . . . 2-14

2-10. Centralization at the Nevada Test Site cumulative shipment-miles for train shipments $\ldots 2-15$

2-11. Comparison of HIGHWAY mileage data with Map-n-Go mileage data $\ldots \ldots \ldots \ldots$. . . . .

2-12. Comparison of INTERLINE mileage data with mileage data from the Railroad Information Service . . . . . . . . . . . . . . . . . . . . . . . 2-17

3-1. Unit risk factors calculated using the RADTRAN 4 computer code $\ldots \ldots \ldots \ldots$. . . 3-2

3-2. Hand-calculated unit risk factors $\ldots \ldots \ldots \ldots \ldots \ldots \ldots \ldots \ldots \ldots \ldots \ldots$

3-3. Percent differences between RADTRAN 4- and hand-calculated unit risk factors ..... 3-3

4-1. RADTRAN 4-calculated and hand-calculated accident risks for case one . . . . . . 4-4

4-2. Conditional probabilities, release fractions, aerosolized fractions, and respirable fractions for case two $\ldots \ldots \ldots \ldots \ldots \ldots \ldots \ldots \ldots \ldots \ldots \ldots \ldots \ldots$

4-3. Radiation doses by pathway and severity class for case two $\ldots \ldots \ldots \ldots \ldots$

4-4. RADTRAN 4-calculated and hand-calculated accident risks for case two . . . . . . . 4-5

5-1. RISKIND-calculated and hand-calculated incident-free radiation doses . . . . . . . . 5-4

6-1. RISKIND-calculated and hand-calculated radiation doses $\ldots \ldots \ldots \ldots \ldots \ldots$. $\ldots \ldots$ 



\section{Validation of the Transportation Computer Codes HIGHWAY, INTERLINE, RADTRAN 4, and RISKIND}

\section{INTRODUCTION}

The computer codes HIGHWAY, INTERLINE, RADTRAN 4, and RISKIND were used to estimate radiation doses from the transportation of radioactive material in DOE (1995). HIGHWAY and INTERLINE were used to estimate transportation routes for truck and rail shipments, respectively. RADTRAN 4 was used to estimate collective doses from incident-free transportation and the risk (probability $\times$ consequence) from transportation accidents. RISKIND was used to estimate incident-free radiation doses for maximally exposed individuals and the consequences from reasonably foreseeable transportation accidents. The purpose of this analysis is to validate the estimates made by these computer codes. Validation was defined as "the test and evaluation of the completed software to ensure compliance with software requirements" (ASME 1989). In the context of this analysis, compliance with software requirements means that the differences between the estimates generated by these codes and independent observations are small (i.e., within the acceptance criterion established for the validation analysis). In some cases, the independent observations used in the validation were measurements; in other cases, the independent observations used in the validation analysis were generated using hand calculations.

Chapter 2 contains the validation analysis for the HIGHWAY and INTERLINE computer codes. Chapter 3 contains the validation analysis for the incident-free unit risk factors calculated using the RADTRAN 4 computer code. Chapter 4 contains the validation analysis for the accident risks calculated using the RADTRAN 4 computer code. Chapter 5 contains the validation analysis for the incident-free radiation doses calculated using the RISKIND computer code. Chapter 6 contains the validation analysis for the accident radiation doses calculated using the RISKIND computer code. 


\section{VALIDATION OF THE HIGHWAY AND INTERLINE RADIOACTIVE MATERIALS TRANSPORTATION ROUTING COMPUTER CODES}

\subsection{Introduction}

The HIGHWAY and INTERLINE computer codes (Johnson et al. 1993a, 1993b) were used to determine the transportation routes for radioactive material shipments in DOE (1995). The purpose of this analysis is to validate the predictions made by HIGHWAY and INTERLINE. The definition of validation used in this analysis was "the test and evaluation of the completed software to ensure compliance with software requirements" (ASME 1989). In the context of this analysis, compliance with software requirements means that the origin to destination distances (predictions) generated by the HIGHWAY and INTERLINE computer codes were compared to origin to destination distances generated using independent data bases (observations); if the differences between the predictions and observations are small (i.e., within the acceptance criterion established for the validation analysis), then the predictions are valid.

The HIGHWAY computer code was used to estimate truck routes and origin to destination distances. HIGHWAY contains a computerized road atlas of the United States that includes the interstate highway system, most U.S. highways, and many state, county and local highways. The HIGHWAY computer code selects transportation routes by minimizing the distance and driving time between an origin and destination. In addition, HIGHWAY can be used to select routes that comply with U.S. Department of Transportation regulations for the routing of radioactive material shipments. HIGHWAY also contains an option to calculate population densities along the route using block group-level data from the 1990 U.S. Census. In the validation analysis, the predictions consisted of origin to destination distances generated using HIGHWAY; the independent observations used for comparison were obtained from the Map-n-Go computer code (Delorme 1994).

The INTERLINE computer code was used to estimate rail routes and origin to destination distances. INTERLINE contains a computerized road atlas of the United States and includes all rail lines with the exception of industrial spurs. The INTERLINE computer code selects transportation routes by preferentially routing shipments along mainlines, while minimizing interchanges between railroads. As with HIGHWAY, INTERLINE also contains an option to calculate population densities along the route using block group-level data from the 1990 U.S. Census. In the validation analysis, 
the predictions consisted of origin to destination distances generated using INTERLINE; the independent observations used for comparison were obtained from the Railroad Information Service (Eggleston 1995).

\subsection{Methods and Materials}

In DOE (1995), approximately 300 truck routes (generated using HIGHWAY) and 300 rail routes (generated using INTERLINE) were evaluated. This large number of routes made it impractical to validate all the routes. In addition, the results presented in DOE (1995) indicate that the five Centralization Alternatives (Centralization at the Hanford Site, the Savannah River Site, the Idaho National Engineering Laboratory, the Oak Ridge Reservation, and the Nevada Test Site) yielded the largest transportation doses and risks for shipments of spent nuclear fuel. Based on these two considerations, a risk-based screening approach was used to select the routes evaluated in the validation analysis.

The risk-based screening approach was based on shipment-miles, the product of the number of shipments from a particular origin to a particular destination and the distance between the origin and destination. Shipment-miles provide an estimate of the total distance traveled by the shipments and are proportional to transportation dose and risk. For each of the five Centralization Alternatives, the shipment-miles were calculated for each origin and destination (see Tables 2-1 through 2-10). Within each Centralization Alternative, the cumulative fraction of the total shipment-miles was calculated for each origin and destination. Routes that contributed greater than 80 cumulative percent of the total shipment-miles for a Centralization Alternative were evaluated in the validation analysis.

For each of the truck routes selected for validation using the risk-based screening approach, the Map-n-Go computer code, an independent highway routing computer code from Delorme Mapping Company (Delorme 1994), was used to calculate the distance from the origin of the route to the destination of the route. For each route, origins and destinations were matched with those used by the HIGHWAY computer code and the routes were selected and distances were calculated independently using the Map-n-Go computer code. In some cases, the Map-n-Go computer code did not contain a node (i.e., an origin or destination) that matched the HIGHWAY node; for example, nodes on U.S. Department of Energy sites. In these cases, HIGHWAY was rerun using a nearby node that was also contained in Map-n-Go data base. Options in Map-n-Go were selected to match the route that would 
be required for a radioactive material shipment; for example, options were selected to favor travel on limited access roads, toll roads, and national highways, and to avoid travel on forest roads and ferries. The distances generated by the Map-n-Go computer code were compared to the distances generated using the HIGHWAY computer code (see Table 2-11).

An alternative computer code for generating rail routes was not available. Instead, the Railroad Information Service was used to generate independent estimates of the rail routes selected for validation using the risk-based screening approach. The Railroad Information Service, located in Georgetown, Texas, is a rail mapping company that owns proprietary rail line data bases and is currently developing rail routing and scheduling software (Eggleston 1995). The Railroad Information Service was provided with node descriptions generated by the INTERLINE computer code for each of the rail routes selected for evaluation in the validation analysis. As with the HIGHWAY computer code, sometimes the Railroad Information Service data base did not contain a node that matched the INTERLINE node (e.g., nodes on U.S. Department of Energy sites). In these cases, INTERLINE was rerun using a nearby node that was also contained in Railroad Information data base. The Railroad Information Service reported the distance results as generated from its independent databases; these distances were compared to the distances generated using the INTERLINE computer code (see Table 2-12).

In order to evaluate the predictions made by HIGHWAY and INTERLINE, a quantitative metric that measures the difference between the predictions and observations was necessary. Many quantitative metrics currently exist, such as the maximum difference, the maximum relative difference, and the relative root mean square error (Baca and Magnuson 1990). In this validation analysis, three metrics were calculated: (1) the difference between the predictions and the observations, (2) the absolute value of the percent difference between the predictions and the observations, and (3) the relative root mean square error (RRMSE) of the predictions and the observations. The absolute difference and the absolute value of the percent difference were calculated in order to provide data on the differences for individual routes. The RRMSE was calculated in order to measure the overall difference between the predictions and observations and was the metric chosen for the acceptance criterion in the validation analysis. The metrics were calculated using the following equations:

Absolute Difference $=$ Predicted distance - Observed distance 


$$
\text { Percent Difference }=\frac{\text { Predicted distance }- \text { Observed distance }}{\text { Predicted distance }} \times 100
$$

$$
\text { RRMSE }=\sqrt{\frac{\sum_{i=1}^{k}\left(\frac{P_{i}-O_{i}}{P_{i}}\right)^{2}}{k}}
$$

where

$$
\begin{array}{lll}
\mathrm{P}_{\mathrm{i}} & = & \text { the predicted distance for route } \mathrm{i} \\
\mathrm{O}_{\mathrm{i}} & = & \text { the observed distance for route } \mathrm{i} \\
\mathrm{k} & = & \text { the number of routes evaluated in the validation analysis. }
\end{array}
$$

The acceptance criterion established for this validation analysis was based on values of the RRMSE (Baca and Magnuson 1990):

$$
\begin{aligned}
& \text { - } \\
& \text { Excellent }- \text { RRMSE } \leq 0.05 \\
& \text { Acceptable }-0.05<\text { RRMSE } \leq 0.10 \\
& \text { Unacceptable - RRMSE }>0.10
\end{aligned}
$$

\subsection{Results}

Table 2-11 presents the results of the validation analysis for the HIGHWAY computer code. The average difference between the predictions and the observations was 21.8 kilometers (13.5 miles). Qualitatively, HIGHWAY did not consistently underestimate or overestimate. This suggests the absence of systematic bias in the predictions made by HIGHWAY. The absolute value of the percent difference between the predictions and the observations was 2.0 percent. A percent difference of 2 percent in the results is not considered to be significant relative to the overall uncertainty in dose assessments, which can have geometric standard deviations that approach 3 (Maheras et al. 1994). The value of the RRMSE was 0.025. Based on the acceptance criterion established for the validation analysis, HIGHWAY yields excellent results. 
Table 2-12 presents the results of the validation analysis for the INTERLINE computer code. The average difference between the predictions and the observations was -13.9 kilometers ( -8.6 miles). The absolute value of the percent difference between the predictions and the observations was 0.60 percent. A percent difference of 0.60 percent in the results is not considered to be significant relative to the overall uncertainty in dose assessments, which can have geometric standard deviations that approach 3 (Maheras et al. 1994). The value of the RRMSE was 0.0074. Based on the acceptance criterion established for the validation analysis, INTERLINE yields excellent results.

\subsection{Conclusions}

A validation analysis was conducted for transportation routes predicted by the HMGHWAY and INTERLINE computer codes. Using a risk-based screening approach based on shipment-miles, the distances predicted by HIGHWAY and INTERLINE were compared to independent observations from the Delorme Mapping Company and the Railroad Information Service. Using the acceptance criterion established for the validation analysis, both HIGHWAY and INTERLINE yielded excellent results.

This demonstrates that HIGHWAY and INTERLINE, within their domain of applicability, adequately represent the systems that they were intended to describe; consequently, the predictions made by HIGHWAY and INTERLINE are considered validated. 
Table 2-1. Centralization at the Hanford Site cumulative shipment-miles for truck shipments. ${ }^{\mathrm{a}}$

\begin{tabular}{|c|c|c|c|c|c|}
\hline & Route & Miles & Shipments & Shipment-miles & $\begin{array}{r}\text { Percent of Cumulative } \\
\text { Shipment-miles }\end{array}$ \\
\hline Hanford Site & Savannah River Site & 2727.0 & 864 & $2.36 \mathrm{E}+06$ & 26.965 \\
\hline Hanford Site & Hampton Roads, VA & 2903.0 & 541 & $1.57 \mathrm{E}+06$ & 44.939 \\
\hline Hanford Site & Idaho National Engineering Laboratory & 599.0 & 1918 & $1.15 \mathrm{E}+06$ & 58.088 \\
\hline Hanford Site & Alexandria Bay, NY & 2768.0 & 236 & $6.53 \mathrm{E}+05$ & 65.564 \\
\hline Hanford Site & Gaithersburg, MD & 2732.0 & 188 & $5.14 \mathrm{E}+05$ & 71.442 \\
\hline Hanford Site & Oak Ridge Reservation & 2464.0 & 120 & $2.96 \mathrm{E}+05$ & 74.826 \\
\hline Hanford Site & Fort St. Vrain Nuclear Generating Station & 1108.0 & 247 & $2.74 \mathrm{E}+05$ & 77.958 \\
\hline Hanford Site & University of Missouri - Columbia & 1870.0 & 145 & $2.71 \mathrm{E}+05$ & 81.061 \\
\hline Hanford Site & West Valley Demonstration Project & 2556.0 & 83 & $2.12 \mathrm{E}+05$ & 83.489 \\
\hline Hanford Site & Massachusetts Institute of Technology & 2986.0 & 69 & $2.06 \mathrm{E}+05$ & 85.847 \\
\hline Hanford Site & Brookhaven National Laboratory & 2853.0 & 71 & $2.03 E+05$ & 88.165 \\
\hline Hanford Site & Oakland, $\mathrm{CA}$ & 875.0 & 231 & $2.02 \mathrm{E}+05$ & 90.479 \\
\hline Hanford Site & University of Michigan & 2227.0 & 72 & $1.60 \mathrm{E}+05$ & 92.314 \\
\hline Hanford Site & Rhode Island Nuclear Science Center & 2965.0 & 32 & $9.49 \mathrm{E}+04$ & 93.400 \\
\hline Hanford Site & Georgia Institute of Technology & 2550.0 & 19 & $4.85 E+04$ & 93.954 \\
\hline Hanford Site & Rensselaer Polytechnic Institute & 2819.0 & 16 & $4.51 \mathrm{E}+04$ & 94.470 \\
\hline Hanford Site & Sandia National Laboratories-Albuquerque & 1584.0 & 27 & $4.28 \mathrm{E}+04$ & 94.960 \\
\hline Hanford Site & Texas A\&M University & 2212.0 & 15 & $3.32 \mathrm{E}+04$ & 95.340 \\
\hline Hanford Site & University of Virginia & 2757.0 & 12 & $3.31 \mathrm{E}+04$ & 95.718 \\
\hline Hanford Site & Cornell University & 2730.0 & 12 & $3.28 \mathrm{E}+04$ & 96.093 \\
\hline Hanford Site & State University of New York - Buffalo & 2534.0 & 12 & $3.04 \mathrm{E}+04$ & 96.441 \\
\hline Hanford Site & Los Alamos National Laboratory & 1560.0 & 17 & $2.65 \mathrm{E}+04$ & 96.745 \\
\hline Hanford Site & University of Illinois & 2033.0 & 13 & $2.64 \mathrm{E}+04$ & 97.047 \\
\hline Hanford Site & North Carolina State University & 2862.0 & 8 & $2.29 \mathrm{E}+04$ & 97.309 \\
\hline Hanford Site & Argonne National Laboratory - East & 1998.0 & 11 & $2.20 \mathrm{E}+04$ & 97.561 \\
\hline Hanford Site & University of Florida & 2894.0 & 7 & $2.03 E+04$ & 97.793 \\
\hline Hanford Site & University of Wisconsin & 1943.0 & 9 & $1.75 \mathrm{E}+04$ & 97.993 \\
\hline Hanford Site & Pennsylvania State University & 2578.0 & 6 & $1.55 \mathrm{E}+04$ & 98.170 \\
\hline Hanford Site & University of Missouri - Rolla & 2082.0 & 7 & $1.46 \mathrm{E}+04$ & 98.336 \\
\hline Hanford Site & University of Maryland & 2753.0 & 5 & $1.38 \mathrm{E}+04$ & 98.494 \\
\hline Hanford Site & University of Lowell & 2991.0 & 4 & $1.20 \mathrm{E}+04$ & 98.631 \\
\hline Hanford Site & Worcester Polytechnic Institute & 2948.0 & 4 & $1.18 \mathrm{E}+04$ & 98.766 \\
\hline Hanford Site & Kansas State University & 1624.0 & 7 & $1.14 \mathrm{E}+04$ & 98.896 \\
\hline Hanford Site & San Diego, CA & 1352.0 & 8 & $1.08 \mathrm{E}+04$ & 99.020 \\
\hline Hanford Site & University of Texas & 2216.0 & 4 & $8.86 \mathrm{E}+03$ & 99.121 \\
\hline Hanford Site & Purdue University & 2111.0 & 4 & $8.44 \mathrm{E}+03$ & 99.218 \\
\hline Hanford Site & Manhattan College & 2786.0 & 3 & $8.36 \mathrm{E}+03$ & 99.314 \\
\hline Hanford Site & Ohio State University & 2342.0 & 3 & $7.03 E+03$ & 99.394 \\
\hline Hanford Site & Midland, MI & 2318.0 & 3 & $6.95 \mathrm{E}+03$ & 99.474 \\
\hline Hanford Site & Iowa State University & 1703.0 & 4 & $6.81 \mathrm{E}+03$ & 99.551 \\
\hline Hanford Site & Denver, $\mathrm{CO}$ & 1133.0 & 6 & $6.80 \mathrm{E}+03$ & 99.629 \\
\hline Hanford Site & Babcock \& Wilcox & 2738.0 & 2 & $5.48 \mathrm{E}+03$ & 99.692 \\
\hline Hanford Site & University of Arizona & 1699.0 & 3 & $5.10 \mathrm{E}+03$ & 99.750 \\
\hline Hanford Site & University of California - Irvine & 1270.0 & 3 & $3.81 \mathrm{E}+03$ & 99.794 \\
\hline Hanford Site & Pleasanton, CA & 881.0 & 4 & $3.52 \mathrm{E}+03$ & 99.834 \\
\hline Hanford Site & Washington State University & 361.0 & 8 & $2.89 \mathrm{E}+03$ & 99.867 \\
\hline Hanford Site & San Ramon,CA & 874.0 & 3 & $2.62 \mathrm{E}+03$ & 99.897 \\
\hline Hanford Site & McClellan AFB, CA & 830.0 & 3 & $2.49 \mathrm{E}+03$ & 99.926 \\
\hline Hanford Site & Oregon State University & 324.0 & 6 & $1.94 \mathrm{E}+03$ & 99.948 \\
\hline Hanford Site & University of Utah & 643.0 & 3 & $1.93 \mathrm{E}+03$ & 99.970 \\
\hline Hanford Site & University of New Mexico & 1593.0 & 1 & $1.59 \mathrm{E} \div 03$ & 99.988 \\
\hline Hanford Site & Idaho State University & 546.0 & 1 & $5.46 \mathrm{E}+02$ & 99.995 \\
\hline Hanford Site & Reed College & 236.0 & 2 & $4.72 \mathrm{E}+02$ & 100.000 \\
\hline Total & & & 5102 & $8.74 E+06$ & \\
\hline
\end{tabular}

a. To convert from miles to kilometers, multiply by 1.6 . 
Table 2-2. Centralization at the Savannah River Site cumulative shipment-miles for truck shipments. ${ }^{a}$

\begin{tabular}{|c|c|c|c|c|c|}
\hline & Route & Miles & Shipments & Shipment-miles & $\begin{array}{r}\text { Percent of Cumulative } \\
\text { Shipment-miles }\end{array}$ \\
\hline Savannah River Site & Hanford Site & 2727.0 & 1716 & $4.68 \mathrm{E}+06$ & 40.219 \\
\hline Savannah River Site & Idaho National Engineering Laboratory & 2311.0 & 1918 & $4.43 E+06$ & 78.314 \\
\hline Savannah River Site & Seattle, WA & 2900.0 & 231 & $6.70 \mathrm{E}+05$ & 84.072 \\
\hline Savannah River Site & Fort St. Vrain Nuclear Generating Station & 1636.0 & 247 & $4.04 \mathrm{E}+05$ & 87.545 \\
\hline Savannah River Site & Hampton Roads, VA & 505.0 & 541 & $2.73 \mathrm{E}+05$ & 89.893 \\
\hline Savannah River Site & Alexandria Bay, $\mathrm{NY}$ & 1012.0 & 236 & $2.39 \mathrm{E}+05$ & 91.946 \\
\hline Savannah River Site & University of Missouri - Columbia & 858.0 & 145 & $1.24 \mathrm{E}+05$ & 93.015 \\
\hline Savannah River Site & Gaithersburg, $\mathrm{MD}$ & 597.0 & 188 & $1.12 \mathrm{E}+05$ & 93.979 \\
\hline Savannah River Site & West Valley Demonstration Project & 883.0 & 83 & $7.33 \mathrm{E}+04$ & 94.609 \\
\hline Savannah River Site & Massachusetts Institute of Technology & 1040.0 & 69 & $7.18 \mathrm{E}+04$ & 95.226 \\
\hline Savannah River Site & University of Michigan & 903.0 & 72 & $6.50 \mathrm{E}+04$ & 95.785 \\
\hline Savannah River Site & Brookhaven National Laboratory & 897.0 & 71 & $6.37 \mathrm{E}+04$ & 96.332 \\
\hline Savannah River Site & Oak Ridge Reservation & 379.0 & 120 & $4.55 \mathrm{E}+04$ & 96.723 \\
\hline Savannah River Site & Sandia National Laboratories-Albuquerque & 1644.0 & 27 & $4.44 \mathrm{E}+04$ & 97.105 \\
\hline Savannah River Site & Rhode Island Nuclear Science Center & 1009.0 & 32 & $3.23 \mathrm{E}+04$ & 97.382 \\
\hline Savannah River Site & Los Alamos National Laboratory & 1742.0 & 17 & $2.96 \mathrm{E}+04$ & 97.637 \\
\hline Savannah River Site & Washington State University & 2699.0 & 8 & $2.16 \mathrm{E}+04$ & 97.822 \\
\hline Savannah River Site & San Diego, $\mathrm{CA}$ & 2345.0 & 8 & $1.88 \mathrm{E}+04$ & 97.983 \\
\hline Savannah River Site & Oregon State University & 2937.0 & 6 & $1.76 \mathrm{E}+04$ & 98.135 \\
\hline Savannah River Site & Texas A\&M University & 1099.0 & 15 & $1.65 \mathrm{E}+04$ & 98.277 \\
\hline Savannah River Site & Rensselaer Polytechnic Institute & 955.0 & 16 & $1.53 \mathrm{E}+04$ & 98.408 \\
\hline Savannah River Site & State University of New York - Buffalo & 1001.0 & 12 & $1.20 \mathrm{E}+04$ & 98.511 \\
\hline Savannah River Site & Pleasanton, $\mathrm{CA}$ & 2768.0 & 4 & $1.11 E+04$ & 98.606 \\
\hline Savannah River Site & Cornell University & 896.0 & 12 & $1.08 \mathrm{E}+04$ & 98.699 \\
\hline Savannah River Site & University of Illinois & 803.0 & 13 & $1.04 \mathrm{E}+04$ & 98.788 \\
\hline Savannah River Site & Argonne National Laboratory - East & 892.0 & 11 & $9.81 \mathrm{E}+03$ & 98.873 \\
\hline Savannah River Site & Denver, $\mathrm{CO}$ & 1613.0 & 6 & $9.68 \mathrm{E}+03$ & 98.956 \\
\hline Savannah River Site & University of Wisconsin & 1038.0 & 9 & $9.34 \mathrm{E}+03$ & 99.036 \\
\hline Savannah River Site & McClellan AFB, CA & 2780.0 & 3 & $8.34 \mathrm{E}+03$ & 99.108 \\
\hline Savannah River Site & San Ramon, CA & 2775.0 & 3 & $8.33 E+03$ & 99.179 \\
\hline Savannah River Site & Kansas State University & 1121.0 & 7 & $7.85 \mathrm{E}+03$ & 99.247 \\
\hline Savannah River Site & University of California - Irvine & 2406.0 & 3 & $7.22 \mathrm{E}+03$ & 99.309 \\
\hline Savannah River Site & University of Utah & 2127.0 & 3 & $6.38 \mathrm{E}+03$ & 99.364 \\
\hline Savannah River Site & University of Missouri - Rolla & 835.0 & 7 & $5.85 \mathrm{E}+03$ & 99.414 \\
\hline Savannah River Site & University of Arizona & 1926.0 & 3 & $5.78 \mathrm{E}+03$ & 99.464 \\
\hline Savannah River Site & University of Virginia & 478.0 & 12 & $5.74 \mathrm{E}+03$ & 99.513 \\
\hline Savannah River Site & Reed College & 2849.0 & 2 & $5.70 \mathrm{E}+03$ & 99.562 \\
\hline Savannah River Site & Pennsylvania State University & 849.0 & 6 & $5.09 \mathrm{E}+03$ & 99.606 \\
\hline Savannah River Site & lowa State University & 1175.0 & 4 & $4.70 \mathrm{E}+03$ & 99.646 \\
\hline Savannah River Site & University of Texas & 1169.0 & 4 & $4.68 \mathrm{E}+03$ & 99.686 \\
\hline Savannah River Site & University of Lowell & 1045.0 & 4 & $4.18 \mathrm{E}+03$ & 99.722 \\
\hline Savannah River Site & Worcester Polytechnic Institute & 1002.0 & 4 & $4.01 \mathrm{E}+03$ & 99.757 \\
\hline Savannah River Site & Georgia Institute of Technology & 197.0 & 19 & $3.74 E+03$ & 99.789 \\
\hline Savannah River Site & University of Florida & 496.0 & 7 & $3.47 \mathrm{E}+03$ & 99.819 \\
\hline Savannah River Site & Midland, MI & 1036.0 & 3 & $3.11 E+03$ & 99.845 \\
\hline Savannah River Site & Purdue University & 768.0 & 4 & $3.07 \mathrm{E}+03$ & 99.872 \\
\hline Savannah River Site & University of Maryland & 589.0 & 5 & $2.95 \mathrm{E}+03$ & 99.897 \\
\hline Savannah River Site & North Carolina State University & 318.0 & 8 & $2.54 \mathrm{E}+03$ & 99.919 \\
\hline Savannah River Site & Manhattan College & 830.0 & 3 & $2.49 \mathrm{E}+03$ & 99.940 \\
\hline Savannah River Site & Idaho State University & 2248.0 & 1 & $2.25 \mathrm{E}+03$ & 99.960 \\
\hline Savannah River Site & Ohio State University & 708.0 & 3 & $2.12 \mathrm{E}+03$ & 99.978 \\
\hline Savannah River Site & University of New Mexico & 1653.0 & 1 & $1.65 \mathrm{E}+03$ & 99.992 \\
\hline Savannah River Site & Babcock \& Wilcox & 455.0 & 2 & $9.10 \mathrm{E}+02$ & 100.000 \\
\hline Total & & & 5954 & $1.16 \mathrm{E}+07$ & \\
\hline
\end{tabular}

a. To convert from miles to kilometers, multiply by 1.6 . 
Table 2-3. Centralization at the Idaho National Engineering Laboratory cumulative shipment-miles for truck shipments.

\begin{tabular}{|c|c|c|c|c|c|}
\hline & Route & Miles & Shipments & Shipment-miles & $\begin{array}{l}\text { Percent of Cumulative } \\
\text { Shipment-miles }\end{array}$ \\
\hline INEL & Savannah River Site & 2311.0 & 864 & $2.00 \mathrm{E}+06$ & 26.882 \\
\hline INEL & Hampton Roads, VA & 2487.0 & 541 & $1.35 \mathrm{E}+06$ & 44.996 \\
\hline INEL & Hanford Site & 599.0 & 1716 & $1.03 E+06$ & 58.835 \\
\hline INEL & Alexandria Bay, $\mathrm{NY}$ & 2352.0 & 236 & $5.55 \mathrm{E}+05$ & 66.307 \\
\hline INEL & Gaithersburg, $\mathrm{MD}$ & 2316.0 & 188 & $4.35 \mathrm{E}+05$ & 72.169 \\
\hline NNEL & Oak Ridge Reservation & 2048.0 & 120 & $2.46 \mathrm{E}+05$ & 75.478 \\
\hline INEL & Oakland, $\mathrm{CA}$ & 963.0 & 231 & $2.22 \mathrm{E} \div 05$ & 78.473 \\
\hline INEL & University of Missouri - Columbia & 1454.0 & 145 & $2.11 E+05$ & 81.311 \\
\hline INEL & West Valley Demonstration Project & 2140.0 & 83 & $1.78 \mathrm{E}+05$ & 83.703 \\
\hline INEL & Massachusetts Institute of Technology & 2570.0 & 69 & $1.77 \mathrm{E}+05$ & 86.090 \\
\hline INEL & Brookhaven National Laboratory & 2437.0 & 71 & $1.73 \mathrm{E}+05$ & 88.420 \\
\hline INEL & Fort St. Vrain Nuclear Generating Station & 692.0 & 247 & $1.71 \mathrm{E}+05$ & 90.721 \\
\hline INEL & University of Michigan & 1811.0 & 72 & $1.30 \mathrm{E}+05$ & 92.476 \\
\hline INEL & Rhode Island Nuclear Science Center & 2549.0 & 32 & $8.16 \mathrm{E}+04$ & 93.574 \\
\hline INEL & Georgia Institute of Technology & 2134.0 & 19 & $4.05 \mathrm{E}+04$ & 94.120 \\
\hline INEL & Rensselaer Polytechnic Institute & 2403.0 & 16 & $3.84 \mathrm{E}+04$ & 94.638 \\
\hline INEL & Sandia National Laboratories-Albuquerque & 1168.0 & 27 & $3.15 E+04$ & 95.063 \\
\hline INEL & University of Virginia & 2341.0 & 12 & $2.81 E+04$ & 95.441 \\
\hline INEL & Comell University & 2314.0 & 12 & $2.78 \mathrm{E}+04$ & 95.815 \\
\hline INEL & Texas A\&M University & 1796.0 & 15 & $2.69 \mathrm{E}+04$ & 96.177 \\
\hline INEL & State University of New York - Buffalo & 2118.0 & 12 & $2.54 \mathrm{E}+04$ & 96.519 \\
\hline INEL & University of Illinois & 1617.0 & 13 & $2.10 \mathrm{E}+04$ & 96.802 \\
\hline INEL & North Carolina State University & 2446.0 & 8 & $1.96 E+04$ & 97.066 \\
\hline INEL & Los Alamos National Laboratory & 1144.0 & 17 & $1.94 \mathrm{E}+04$ & 97.328 \\
\hline INEL & Argonne National Laboratory - East & 1582.0 & 11 & $1.74 \mathrm{E}+04$ & 97.562 \\
\hline INEL & University of Florida & 2478.0 & 7 & $1.73 E+04$ & 97.796 \\
\hline INEL & University of Wisconsin & 1612.0 & 9 & $1.45 \mathrm{E}+04$ & 97.991 \\
\hline INEL & Pennsylvania State University & 2162.0 & 6 & $1.30 \mathrm{E}+04$ & 98.166 \\
\hline INEL & University of Maryland & 2337.0 & 5 & $1.17 \mathrm{E}+04$ & 98.323 \\
\hline INEL & University of Missouri - Rolla & 1666.0 & 7 & $1.17 \mathrm{E}+04$ & 98.480 \\
\hline INEL & University of Lowell & 2575.0 & 4 & $1.03 \mathrm{E}+04$ & 98.619 \\
\hline INEL & Worcester Polytechnic Institute & 2532.0 & 4 & $1.01 \mathrm{E}+04$ & 98.755 \\
\hline INEL & Kansas State University & 1208.0 & 7 & $8.46 \mathrm{E}+03$ & 98.869 \\
\hline INEL & San Diego, CA & 976.0 & 8 & $7.81 \mathrm{E}+03$ & 98.974 \\
\hline NEL & University of Texas & 1800.0 & 4 & $7.20 \mathrm{E}+03$ & 99.071 \\
\hline INEL & Manhattan College & 2370.0 & 3 & $7.11 \mathrm{E}+03$ & 99.167 \\
\hline INEL & Purdue University & 1695.0 & 4 & $6.78 \mathrm{E}+03$ & 99.258 \\
\hline INEL & Ohio State University & 1926.0 & 3 & $5.78 \mathrm{E} \div 03$ & 99.336 \\
\hline INEL & Midland, MI & 1902.0 & 3 & $5.71 \mathrm{E}+03$ & 99.412 \\
\hline INEL & Washington State University & 652.0 & 8 & $5.22 \mathrm{E}+03$ & 99.483 \\
\hline INEL & Iowa State University & 1287.0 & 4 & $5.15 E+03$ & 99.552 \\
\hline INEL & Oregon State University & 809.0 & 6 & $4.85 \mathrm{E}+03$ & 99.617 \\
\hline INEL & Babcock \& Wilcox & 2322.0 & 2 & $4.64 \mathrm{E}+03$ & 99.680 \\
\hline INEL & Denver, $\mathrm{CO}$ & 717.0 & 6 & $4.30 \mathrm{E}+03$ & 99.738 \\
\hline INEL & University of Arizona & 1301.0 & 3 & $3.90 \mathrm{E}+03$ & 99.790 \\
\hline INEL & Pleasanton, $\mathrm{CA}$ & 969.0 & 4 & $3.88 \mathrm{E}+03$ & 99.842 \\
\hline INEL & San Ramon, CA & 962.0 & 3 & $2.89 \mathrm{E}+03$ & 99.881 \\
\hline INEL & University of California - Irvine & 942.0 & 3 & $2.83 \mathrm{E}+03$ & 99.919 \\
\hline INEL & McClellan AFB, CA & 875.0 & 3 & $2.63 \mathrm{E}+03$ & 99.955 \\
\hline INEL & Reed College & 721.0 & 2 & $1.44 \mathrm{E}+03$ & 99.974 \\
\hline INEL & University of New Mexico & 1177.0 & 1 & $1.18 \mathrm{E}+03$ & 99.990 \\
\hline INEL & University of Utah & 227.0 & 3 & $6.81 \mathrm{E}+02$ & 99.999 \\
\hline INEL & Idaho State University & 65.0 & 1 & $6.50 \mathrm{E}+01$ & 100.000 \\
\hline Total & & & 4900 & $7.43 \mathrm{E}+06$ & \\
\hline
\end{tabular}

a. To convert from miles to kilometers, multiply by 1.6 . 
Table 2-4. Centralization at the Oak Ridge Reservation cumulative shipment-miles for truck shipments.

\begin{tabular}{|c|c|c|c|c|}
\hline Route & Miles & Shipments & Shipment-miles & $\begin{array}{l}\text { Percent of Cumulative } \\
\text { Shipment-miles }\end{array}$ \\
\hline Oak Ridge Reservation & 2464.0 & 1716 & $4.23 \mathrm{E}+06$ & 39.538 \\
\hline Idaho National Engineering Laboratory & 2048.0 & 1918 & $3.93 \mathrm{E}+06$ & 76.270 \\
\hline Oak Ridge Reservation & 2636.0 & 231 & $6.09 \mathrm{E}+05$ & 81.964 \\
\hline Fort St. Vrain Nuclear Generating Station & 1372.0 & 247 & $3.39 \mathrm{E}+05$ & 85.133 \\
\hline Oak Ridge Reservation & 379.0 & 864 & $3.27 \mathrm{E}+05$ & 88.195 \\
\hline Oak Ridge Reservation & 548.0 & 541 & $2.96 \mathrm{E}+05$ & 90.967 \\
\hline Oak Ridge Reservation & 927.0 & 236 & $2.19 \mathrm{E}+05$ & 93.013 \\
\hline Oak Ridge Reservation & 536.0 & 188 & $1.01 \mathrm{E}+05$ & 93.955 \\
\hline University of Missouri - Columbia & 594.0 & 145 & $8.61 E+04$ & 94.761 \\
\hline Massachusetts Institute of Technology & 965.0 & 69 & $6.66 \mathrm{E}+04$ & 95.384 \\
\hline West Valley Demonstration Project & 766.0 & 83 & $6.36 \mathrm{E}+04$ & 95.978 \\
\hline Brookhaven National Laboratory & 821.0 & 71 & $5.83 E+04$ & 96.523 \\
\hline University of Michigan & 595.0 & 72 & $4.28 \mathrm{E}+04$ & 96.924 \\
\hline Sandia National Laboratories-Albuquerque & 1382.0 & 27 & $3.73 \mathrm{E}+04$ & 97.273 \\
\hline Rhode Island Nuclear Science Center & 933.0 & 32 & $2.99 \mathrm{E}+04$ & 97.552 \\
\hline Los Alamos National Laboratory & 1480.0 & 17 & $2.52 \mathrm{E}+04$ & 97.787 \\
\hline Washington State University & 2435.0 & 8 & $1.95 \mathrm{E}+04$ & 97.969 \\
\hline Oak Ridge Reservation & 2193.0 & 8 & $1.75 \mathrm{E}+04$ & 98.133 \\
\hline Oregon State University & 2674.0 & 6 & $1.60 \mathrm{E}+04$ & 98.283 \\
\hline Texas A\&M University & 1004.0 & 15 & $1.51 \mathrm{E}+04$ & 98.424 \\
\hline Rensselaer Polytechnic Institute & 879.0 & 16 & $1.41 E+04$ & 98.556 \\
\hline Oak Ridge Reservation & 2532.0 & 4 & $1.01 E+04$ & 98.650 \\
\hline Oak Ridge Reservation & 821.0 & 12 & $9.85 \mathrm{E}+03$ & 98.743 \\
\hline State University of New York - Buffalo & 744.0 & 12 & $8.93 E+03$ & 98.826 \\
\hline Oak Ridge Reservation & 1340.0 & 6 & $8.04 E+03$ & 98.901 \\
\hline Oak Ridge Reservation & 2538.0 & 3 & $7.61 E+03$ & 98.972 \\
\hline Oak Ridge Reservation & 2517.0 & 3 & $7.55 \mathrm{E}+03$ & 99.043 \\
\hline Oak Ridge Reservation & 516.0 & 13 & $6.71 \mathrm{E}+03$ & 99.106 \\
\hline University of California - Irvine & 2209.0 & 3 & $6.63 \mathrm{E}+03$ & 99.168 \\
\hline University of Wisconsin & 730.0 & 9 & $6.57 \mathrm{E}+03$ & 99.229 \\
\hline Argonne National Laboratory - East & 584.0 & 11 & $6.42 \mathrm{E}+03$ & 99.289 \\
\hline Kansas State University & 857.0 & 7 & $6.00 \mathrm{E}+03$ & 99.345 \\
\hline Oak Ridge Reservation & 1864.0 & 3 & $5.59 \mathrm{E}+03$ & 99.398 \\
\hline Oak Ridge Reservation & 1782.0 & 3 & $5.35 \mathrm{E}+03$ & 99.448 \\
\hline Oak Ridge Reservation & 2585.0 & 2 & $5.17 \mathrm{E}+03$ & 99.496 \\
\hline Oak Ridge Reservation & 402.0 & 12 & $4.82 \mathrm{E}+03$ & 99.541 \\
\hline Pennsylvania State University & 774.0 & 6 & $4.64 \mathrm{E}+03$ & 99.585 \\
\hline Oak Ridge Reservation & 1026.0 & 4 & $4.10 \mathrm{E}+03$ & 99.623 \\
\hline University of Missouri - Rolla & 571.0 & 7 & $4.00 \mathrm{E}+03$ & 99.660 \\
\hline University of Lowell & 970.0 & 4 & $3.88 \mathrm{E}+03$ & 99.697 \\
\hline Georgia Institute of Technology & 202.0 & 19 & $3.84 \mathrm{E}+03$ & 99.732 \\
\hline University of Florida & 546.0 & 7 & $3.82 \mathrm{E}+03$ & 99.768 \\
\hline Oak Ridge Reservation Worcester Polytechnic Institute & 927.0 & 4 & $3.71 \mathrm{E} \div 03$ & 99.803 \\
\hline Iowa State University & 900.0 & 4 & $3.60 \mathrm{E}+03$ & 99.837 \\
\hline North Carolina State University & 408.0 & 8 & $3.26 \mathrm{E}+03$ & 99.867 \\
\hline Oak Ridge Reservation University of Maryland & 537.0 & 5 & $2.69 \mathrm{E} \div 03$ & 99.892 \\
\hline Oak Ridge Reservation & 754.0 & 3 & $2.26 \mathrm{E} \div 03$ & 99.913 \\
\hline Oak Ridge Reservation Midiand, MI & 719.0 & 3 & $2.16 \mathrm{E}+03$ & 99.933 \\
\hline Oak Ridge Reservation Idaho State University & 1985.0 & 1 & $1.99 \mathrm{E}+03$ & 99.952 \\
\hline Oak Ridge Reservation & 460.0 & 4 & $1.84 \mathrm{E}+03$ & 99.969 \\
\hline Oak Ridge Reservation University of New Mexico & 1391.0 & 1 & $1.39 \mathrm{E}+03$ & 99.982 \\
\hline Oak Ridge Reservation Ohio State University & 400.0 & 3 & $1.20 \mathrm{E}+03$ & 99.993 \\
\hline Oak Ridge Reservation Babcock \& Wilcox & 350.0 & 2 & $7.00 \mathrm{E}+02$ & 100.000 \\
\hline Total & & 6698 & $1.07 \mathrm{E}+07$ & \\
\hline
\end{tabular}


Table 2-5. Centralization at the Nevada Test Site cumulative shipment-miles for truck shipments. ${ }^{\text {a }}$

\begin{tabular}{|c|c|c|c|c|c|}
\hline & Route & Miles & Shipments & Shipment-miles & $\begin{array}{l}\text { Percent of Cumulative } \\
\text { Shipment-miles }\end{array}$ \\
\hline Nevada Test Site & Savannah River Site & 2414.0 & 864 & $2.09 \mathrm{E}+06$ & 20.469 \\
\hline Nevada Test Site & Hanford Site & 1128.0 & 1716 & $1.94 E+06$ & 39.466 \\
\hline Nevada Test Site & Hampton Roads, VA & 2590.0 & 541 & $1.40 \mathrm{E}+06$ & 53.217 \\
\hline Nevada Test Site & Idaho National Engineering Laboratory & 712.0 & 1918 & $1.37 \mathrm{E}+06$ & 66.620 \\
\hline Nevada Test Site & Alexandria Bay, NY & 2619.0 & 236 & $6.18 \mathrm{E}+05$ & 72.685 \\
\hline Nevada Test Site & Gaithersburg, MD & 2488.0 & 188 & $4.68 \mathrm{E}+05$ & 77.276 \\
\hline Nevada Test Site & Seattle, WA & 1322.0 & 231 & $3.05 \mathrm{E}+05$ & 80.273 \\
\hline Nevada Test Site & Oak Ridge Reservation & 2151.0 & 120 & $2.58 \mathrm{E}+05$ & 82.806 \\
\hline Nevada Test Site & University of Missouri - Columbia & 1557.0 & 145 & $2.26 \mathrm{E}+05$ & 85.022 \\
\hline Nevada Test Site & Fort St. Vrain Nuclear Generating Station & 852.0 & 247 & $2.10 \mathrm{E}+05$ & 87.087 \\
\hline Nevada Test Site & West Valley Demonstration Project & 2373.0 & 83 & $1.97 \mathrm{E}+05$ & 89.020 \\
\hline Nevada Test Site & Massachusetts Institute of Technology & 2802.0 & 69 & $1.93 \mathrm{E}+05$ & 90.918 \\
\hline Nevada Test Site & Brookhaven National Laboratory & 2670.0 & 71 & $1.90 \mathrm{E}+05$ & 92.778 \\
\hline Nevada Test Site & University of Michigan & 2044.0 & 72 & $1.47 \mathrm{E}+05$ & 94.222 \\
\hline Nevada Test Site & Rhode Island Nuclear Science Center & 2782.0 & 32 & $8.90 \mathrm{E}+04$ & 95.096 \\
\hline Nevada Test Site & Georgia Institute of Technology & 2238.0 & 19 & $4.25 \mathrm{E}+04$ & 95.513 \\
\hline Nevada Test Site & Rensselaer Polytechnic Institute & 2636.0 & 16 & $4.22 \mathrm{E}+04$ & 95.927 \\
\hline Nevada Test Site & Cornell University & 2547.0 & 12 & $3.06 \mathrm{E}+04$ & 96.227 \\
\hline Nevada Test Site & University of Virginia & 2444.0 & 12 & $2.93 \mathrm{E}+04$ & 96.515 \\
\hline Nevada Test Site & State University of New York - Buffalo & 2350.0 & 12 & $2.82 E+04$ & 96.792 \\
\hline Nevada Test Site & Texas A\&M University & 1852.0 & 15 & $2.78 \mathrm{E}+04$ & 97.065 \\
\hline Nevada Test Site & Sandia National Laboratories-Albuquerque & 909.0 & 27 & $2.45 \mathrm{E}+04$ & 97.305 \\
\hline Nevada Test Site & University of Illinois & 1850.0 & 13 & $2.41 E+04$ & 97.541 \\
\hline Nevada Test Site & North Carolina State University & 2549.0 & 8 & $2.04 \mathrm{E}+04$ & 97.742 \\
\hline Nevada Test Site & Argonne National Laboratory - East & 1815.0 & 11 & $2.00 \mathrm{E}+04$ & 97.937 \\
\hline Nevada Test Site & University of Florida & 2582.0 & 7 & $1.81 E+04$ & 98.115 \\
\hline Nevada Test Site & Los Alamos National Laboratory & 997.0 & 17 & $1.69 \mathrm{E}+04$ & 98.281 \\
\hline Nevada Test Site & University of Wisconsin & 1857.0 & 9 & $1.67 \mathrm{E}+04$ & 98.445 \\
\hline Nevada Test Site & Pennsylvania State University & 2395.0 & 6 & $1.44 \mathrm{E}+04$ & 98.586 \\
\hline Nevada Test Site & University of Maryland & 2509.0 & 5 & $1.25 \mathrm{E}+04$ & 98.709 \\
\hline Nevada Test Site & University of Missouri - Rolla & 1769.0 & 7 & $1.24 \mathrm{E}+04$ & 98.831 \\
\hline Nevada Test Site & University of Lowell & 2808.0 & 4 & $1.12 \mathrm{E}+04$ & 98.941 \\
\hline Nevada Test Site & Worcester Polytechnic Institute & 2765.0 & 4 & $1.11 \mathrm{E}+04$ & 99.050 \\
\hline Nevada Test Site & Washington State University & 1286.0 & 8 & $1.03 \mathrm{E}+04$ & 99.151 \\
\hline Nevada Test Site & Kansas State University & 1312.0 & 7 & $9.18 \mathrm{E}+03$ & 99.241 \\
\hline Nevada Test Site & Manhattan College & 2603.0 & 3 & $7.81 \mathrm{E}+03$ & 99.317 \\
\hline Nevada Test Site & Purdue University & 1928.0 & 4 & $7.71 \mathrm{E}+03$ & 99.393 \\
\hline Nevada Test Site & Oregon State University & 1245.0 & 6 & $7.47 \mathrm{E}+03$ & 99.466 \\
\hline Nevada Test Site & University of Texas & 1662.0 & 4 & $6.65 \mathrm{E}+03$ & 99.532 \\
\hline Nevada Test Site & Midland, MI & 2135.0 & 3 & $6.41 \mathrm{E}+03$ & 99.595 \\
\hline Nevada Test Site & Ohio State University & 2098.0 & 3 & $6.29 \mathrm{E}+03$ & 99.656 \\
\hline Nevada Test Site & Iowa State University & 1520.0 & 4 & $6.08 \mathrm{E}+03$ & 99.716 \\
\hline Nevada Test Site & Babcock \& Wilcox & 2491.0 & 2 & $4.98 \mathrm{E}+03$ & 99.765 \\
\hline Nevada Test Site & Denver, $\mathrm{CO}$ & 819.0 & 6 & $4.91 \mathrm{E}+03$ & 99.813 \\
\hline Nevada Test Site & San Diego, CA & 398.0 & 8 & $3.18 \mathrm{E}+03$ & 99.844 \\
\hline Nevada Test Site & Pleasanton, CA & 697.0 & 4 & $2.79 E+03$ & 99.872 \\
\hline Nevada Test Site & Reed College & 1250.0 & 2 & $2.50 \mathrm{E}+03$ & 99.896 \\
\hline Nevada Test Site & McClellan AFB, CA & 735.0 & 3 & $2.21 \mathrm{E}+03$ & 99.918 \\
\hline Nevada Test Site & University of Arizona & 723.0 & 3 & $2.17 \mathrm{E}+03$ & 99.939 \\
\hline Nevada Test Site & San Ramon, CA & 694.0 & 3 & $2.08 \mathrm{E}+03$ & 99.960 \\
\hline Nevada Test Site & University of Utah & 487.0 & 3 & $1.46 \mathrm{E}+03$ & 99.974 \\
\hline Nevada Test Site & University of California - Irvine & 364.0 & 3 & $1.09 \mathrm{E}+03$ & 99.985 \\
\hline Nevada Test Site & University of New Mexico & 918.0 & 1 & $9.18 \mathrm{E}+02$ & 99.994 \\
\hline Nevada Test Site & Idaho State University & 649.0 & 1 & $6.49 \mathrm{E}+02$ & 100.000 \\
\hline Total & & & 6818 & $1.02 \mathrm{E}+07$ & \\
\hline
\end{tabular}

a. To convert from miles to kilometers, multiply by 1.6 . 
Table 2-6. Centralization at the Hanford Site cumulative shipment-miles for train shipments.

\begin{tabular}{|c|c|c|c|c|c|}
\hline & Route & Miles & Shipments & Shipment-miles & $\begin{array}{l}\text { Percent of Cumulative } \\
\text { Shipment-miles }\end{array}$ \\
\hline Hanford Site & Military Ocean Terminal Sunny Point, NC & 3203.0 & 541 & $1.73 \mathrm{E}+06$ & 31.974 \\
\hline Hanford Site & Gouvenour, NY & 2878.0 & 236 & $6.79 \mathrm{E}+05$ & 44.506 \\
\hline Hanford Site & Gaithersburg, MD & 2881.0 & 188 & $5.42 \mathrm{E}+05$ & 54.500 \\
\hline Hanford Site & Savannah River Site & 2953.0 & 173 & $5.11 \mathrm{E}+05$ & 63.926 \\
\hline Hanford Site & University of Missouri - Columbia & 1948.0 & 145 & $2.82 \mathrm{E}+05$ & 69.138 \\
\hline Hanford Site & Idaho National Engineering Laboratory & 658.0 & 366 & $2.41 \mathrm{E}+05$ & 73.582 \\
\hline Hanford Site & Oakland, $\mathrm{CA}$ & 986.0 & 231 & $2.28 \mathrm{E}+05$ & 77.785 \\
\hline Hanford Site & Massachusetts Institute of Technology & 3105.0 & 69 & $2.14 \mathrm{E}+05$ & 81.738 \\
\hline Hanford Site & University of Michigan & 2369.0 & 72 & $1.71 \mathrm{E}+05$ & 84.885 \\
\hline Hanford Site & Rhode Island Nuclear Science Center & 3166.0 & 32 & $1.01 E+05$ & 86.754 \\
\hline Hanford Site & Oak Ridge Reservation & 2601.0 & 26 & $6.76 \mathrm{E}+04$ & 88.002 \\
\hline Hanford Site & Georgia Institute of Technology & 2732.0 & 19 & $5.19 \mathrm{E}+04$ & 88.960 \\
\hline Hanford Site & Rensselaer Polytechnic Institute & 2934.0 & 16 & $4.69 \mathrm{E}+04$ & 89.826 \\
\hline Hanford Site & Texas A\&M University & 2954.0 & 15 & $4.43 \mathrm{E}+04$ & 90.644 \\
\hline Hanford Site & Brookhaven National Laboratory & 3153.0 & 14 & $4.41 \mathrm{E}+04$ & 91.458 \\
\hline Hanford Site & Fort St. Vrain Nuclear Generating Station & 1218.0 & 35 & $4.26 \mathrm{E}+04$ & 92.245 \\
\hline Hanford Site & University of Virginia & 2902.0 & 12 & $3.48 \mathrm{E}+04$ & 92.887 \\
\hline Hanford Site & Cornell University & 2842.0 & 12 & $3.41 \mathrm{E}+04$ & 93.517 \\
\hline Hanford Site & State University of New York - Buffalo & 2637.0 & 12 & $3.16 \mathrm{E}+04$ & 94.101 \\
\hline Hanford Site & University of Illinois & 2158.0 & 13 & $2.81 \mathrm{E}+04$ & 94.618 \\
\hline Hanford Site & North Carolina State University & 3172.0 & 8 & $2.54 \mathrm{E}+04$ & 95.087 \\
\hline Hanford Site & University of Florida & 3138.0 & 7 & $2.20 \mathrm{E}+04$ & 95.492 \\
\hline Hanford Site & University of Wisconsin & 2210.0 & 9 & $1.99 \mathrm{E}+04$ & 95.859 \\
\hline Hanford Site & Pennsylvania State University & 2760.0 & 6 & $1.66 \mathrm{E}+04$ & 96.164 \\
\hline Hanford Site & University of Missouri - Rolla & 2246.0 & 7 & $1.57 \mathrm{E}+04$ & 96.454 \\
\hline Hanford Site & University of Maryland & 2900.0 & 5 & $1.45 \mathrm{E}+04$ & 96.722 \\
\hline Hanford Site & San Diego, CA & 1622.0 & 8 & $1.30 \mathrm{E}+04$ & 96.961 \\
\hline Hanford Site & University of Lowell & 3095.0 & 4 & $1.24 \mathrm{E}+04$ & 97.190 \\
\hline Hanford Site & Worcester Polytechnic Institute & 3089.0 & 4 & $1.24 \mathrm{E}+04$ & 97.418 \\
\hline Hanford Site & Kansas State University & 1743.0 & 7 & $1.22 \mathrm{E}+04$ & 97.643 \\
\hline Hanford Site & Sandia National Laboratories-Albuquerque & 1793.0 & 6 & $1.08 \mathrm{E}+04$ & 97.842 \\
\hline Hanford Site & West Valley Demonstration Project & 2654.0 & 4 & $1.06 \mathrm{E}+04$ & 98.037 \\
\hline Hanford Site & University of Texas & 2473.0 & 4 & $9.89 \mathrm{E}+03$ & 98.220 \\
\hline Hanford Site & Purdue University & 2359.0 & 4 & $9.44 \mathrm{E}+03$ & 98.394 \\
\hline Hanford Site & Manhattan College & 3070.0 & 3 & $9.21 \mathrm{E}+03$ & 98.564 \\
\hline Hanford Site & Denver, $\mathrm{CO}$ & 1254.0 & 6 & $7.52 \mathrm{E}+03$ & 98.703 \\
\hline Hanford Site & Midland, MI & 2507.0 & 3 & $7.52 \mathrm{E}+03$ & 98.842 \\
\hline Hanford Site & Ohio State University & 2482.0 & 3 & $7.45 \mathrm{E}+03$ & 98.979 \\
\hline Hanford Site & Iowa State University & 1788.0 & 4 & $7.15 \mathrm{E}+03$ & 99.111 \\
\hline Hanford Site & Los Alamos National Laboratory & 1725.0 & 4 & $6.90 \mathrm{E}+03$ & 99.238 \\
\hline Hanford Site & Argonne National Laboratory - East & 2200.0 & 3 & $6.60 \mathrm{E}+03$ & 99.360 \\
\hline Hanford Site & Babcock \& Wilcox & 2879.0 & 2 & $5.76 \mathrm{E}+03$ & 99.466 \\
\hline Hanford Site & University of Arizona & 1804.0 & 3 & $5.41 \mathrm{E}+03$ & 99.566 \\
\hline Hanford Site & University of California - Irvine & 1528.0 & 3 & $4.58 \mathrm{E}+03$ & 99.651 \\
\hline Hanford Site & Pleasanton, $\mathrm{CA}$ & 1002.0 & 4 & $4.01 \mathrm{E}+03$ & 99.725 \\
\hline Hanford Site & San Ramon, CA & 1002.0 & 3 & $3.01 \mathrm{E}+03$ & 99.780 \\
\hline Hanford Site & McClellan AFB, CA & 890.0 & 3 & $2.67 \mathrm{E}+03$ & 99.829 \\
\hline Hanford Site & University of Utah & 774.0 & 3 & $2.32 \mathrm{E}+03$ & 99.872 \\
\hline Hanford Site & Oregon State University & 340.0 & 6 & $2.04 \mathrm{E}+03$ & 99.910 \\
\hline Hanford Site & Washington State University & 251.0 & 8 & $2.01 E+03$ & 99.947 \\
\hline Hanford Site & University of New Mexico & 1796.0 & 1 & $1.80 \mathrm{E}+03$ & 99.980 \\
\hline Hanford Site & Idaho State University & 602.0 & 1 & $6.02 \mathrm{E}+02$ & 99.991 \\
\hline Hanford Site & Reed College & 239.0 & 2 & $4.78 \mathrm{E}+02$ & 100.000 \\
\hline Total & & & 2375 & $5.42 \mathrm{E}+06$ & \\
\hline
\end{tabular}

a. To convert from miles to kilometers, multiply by 1.6 . 
Table 2-7. Centralization at the Savannah River Site cumulative shipment-miles for train shipments. ${ }^{\mathrm{a}}$

\begin{tabular}{|c|c|c|c|c|c|}
\hline & Route & Miles & Shipments & Shipment-miles & $\begin{array}{r}\text { Percent of Cumulative } \\
\text { Shipment-miles }\end{array}$ \\
\hline Savannah River Site & Hanford Site & 2953.0 & 646 & $1.91 \mathrm{E}+06$ & 38.105 \\
\hline Savannah River Site & Idaho National Engineering Laboratory & 2407.0 & 366 & $8.81 E+05$ & 55.702 \\
\hline Savannah River Site & Oakland, CA & 3192.0 & 231 & $7.37 \mathrm{E}+05$ & 70.431 \\
\hline Savannah River Site & Gouvenour, NY & 1281.0 & 236 & $3.02 \mathrm{E}+05$ & 76.470 \\
\hline Savannah River Site & Hampton Roads, VA & 529.0 & 541 & $2.86 \mathrm{E}+05$ & 82.186 \\
\hline Savannah River Site & University of Missouri - Columbia & 1011.0 & 145 & $1.47 \mathrm{E}+05$ & 85.115 \\
\hline Savannah River Site & Gaithersburg, MD & 659.0 & 188 & $1.24 \mathrm{E}+05$ & 87.589 \\
\hline Savannah River Site & Massachusetts Institute of Technology & 1223.0 & 69 & $8.44 E+04$ & 89.275 \\
\hline Savannah River Site & University of Michigan & 913.0 & 72 & $6.57 \mathrm{E}+04$ & 90.588 \\
\hline Savannah River Site & Fort St. Vrain Nuclear Generating Station & 1853.0 & 35 & $6.49 \mathrm{E}+04$ & 91.883 \\
\hline Savannah River Site & Rhode Island Nuclear Science Center & 1252.0 & 32 & $4.01 E+04$ & 92.684 \\
\hline Savannah River Site & San Diego, CA & 3274.0 & 8 & $2.62 \mathrm{E}+04$ & 93.207 \\
\hline Savannah River Site & Washington State University & 2864.0 & 8 & $2.29 \mathrm{E}+04$ & 93.665 \\
\hline Savannah River Site & Oregon State University & 3381.0 & 6 & $2.03 E+04$ & 94.070 \\
\hline Savannah River Site & Texas A\&M University & 1194.0 & 15 & $1.79 \mathrm{E}+04$ & 94.428 \\
\hline Savannah River Site & Brookhaven National Laboratory & 1239.0 & 14 & $1.73 \mathrm{E}+04$ & 94.774 \\
\hline Savannah River Site & Rensselaer Polytechnic Institute & 1044.0 & 16 & $1.67 \mathrm{E}+04$ & 95.108 \\
\hline Savannah River Site & Sandia National Laboratories-Albuquerque & 2315.0 & 6 & $1.39 \mathrm{E}+04$ & 95.385 \\
\hline Savannah River Site & University of Illinois & 1028.0 & 13 & $1.34 \mathrm{E}+04$ & 95.652 \\
\hline Savannah River Site & Cornell University & 1098.0 & 12 & $1.32 \mathrm{E}+04$ & 95.915 \\
\hline Savannah River Site & Denver, $\mathrm{CO}$ & 2125.0 & 6 & $1.28 \mathrm{E}+04$ & 96.170 \\
\hline Savannah River Site & Pleasanton, $\mathrm{CA}$ & 3170.0 & 4 & $1.27 \mathrm{E}+04$ & 96.423 \\
\hline Savannah River Site & State University of New York - Buffalo & 1051.0 & 12 & $1.26 \mathrm{E} \div 04$ & 96.675 \\
\hline Savannah River Site & Oak Ridge Reservation & 417.0 & 26 & $1.08 \mathrm{E}+04$ & 96.892 \\
\hline Savannah River Site & University of Wisconsin & 1092.0 & 9 & $9.83 \mathrm{E}+03$ & 97.088 \\
\hline Savannah River Site & University of California - Irvine & 3180.0 & 3 & $9.54 \mathrm{E}+03$ & 97.279 \\
\hline Savannah River Site & San Ramon, CA & 3170.0 & 3 & $9.51 E+03$ & 97.469 \\
\hline Savannah River Site & McClellan AFB, CA & 3160.0 & 3 & $9.48 E+03$ & 97.658 \\
\hline Savannah River Site & Los Alamos National Laboratory & 2252.0 & 4 & $9.01 E+03$ & 97.838 \\
\hline Savannah River Site & Kansas State University & 1274.0 & 7 & $8.92 \mathrm{E}+03$ & 98.016 \\
\hline Savannah River Site & University of Virginia & 637.0 & 12 & $7.64 \mathrm{E}+03$ & 98.169 \\
\hline Savannah River Site & University of Utah & 2378.0 & 3 & $7.13 E+03$ & 98.311 \\
\hline Savannah River Site & University of Missouri - Rolla & 966.0 & 7 & $6.76 \mathrm{E}+03$ & 98.446 \\
\hline Savannah River Site & University of Arizona & 2245.0 & 3 & $6.74 \mathrm{E}+03$ & 98.581 \\
\hline Savannah River Site & Reed College & 3154.0 & 2 & $6.31 E+03$ & 98.707 \\
\hline Savannah River Site & Pennsylvania State University & 963.0 & 6 & $5.78 \mathrm{E}+03$ & 98.822 \\
\hline Savannah River Site & University of Texas & 1314,0 & 4 & $5.26 \mathrm{E}+03$ & 98.927 \\
\hline Savannah River Site & Iowa State University & 1281.0 & 4 & $5.12 E+03$ & 99.030 \\
\hline Savannah River Site & University of Lowell & 1239.0 & 4 & $4.96 \mathrm{E}+03$ & 99.129 \\
\hline Savannah River Site & West Valley Demonstration Project & 1217.0 & 4 & $4.87 \mathrm{E}+03$ & 99.226 \\
\hline Savannah River Site & Worcester Polytechnic Institute & 1176.0 & 4 & $4.70 \mathrm{E}+03$ & 99.320 \\
\hline Savannah River Site & Georgia Instinite of Technology & 221.0 & 19 & $4.20 \mathrm{E}+03$ & 99.404 \\
\hline Savannah River Site & Purdue University & 903.0 & 4 & $3.61 \mathrm{E}+03$ & 99.476 \\
\hline Savannah River Site & Manhattan College & 1156.0 & 3 & $3.47 \mathrm{E}+03$ & 99.545 \\
\hline Savannah River Site & University of Maryland & 669.0 & 5 & $3.35 \mathrm{E}+03$ & 99.612 \\
\hline Savannah River Site & North Carolina State University & 385.0 & 8 & $3.08 \mathrm{E}+03$ & 99.673 \\
\hline Savannah River Site & Midland, MI & 996.0 & 3 & $2.99 \mathrm{E}+03$ & 99.733 \\
\hline Savannah River Site & Argonne National Laboratory - East & 976.0 & 3 & $2.93 \mathrm{E}+03$ & 99.792 \\
\hline Savannah River Site & Idaho State University & 2323,0 & 1 & $2.32 E+03$ & 99.838 \\
\hline Savannah River Site & University of New Mexico & 2315.0 & 1 & $2.32 \mathrm{E}+03$ & 99.884 \\
\hline Savannah River Site & University of Florida & 328.0 & 7 & $2.30 \mathrm{E}+03$ & 99.930 \\
\hline Savannah River Site & Ohio State University & 726.0 & 3 & $2.18 \mathrm{E}+03$ & 99.974 \\
\hline Savannah River Site & Babcock \& Wilcox & 661.0 & 2 & $1.32 \mathrm{E}+03$ & 100.000 \\
\hline Total & & & 2848 & $5.01 E+06$ & \\
\hline
\end{tabular}

a. To convert from miles to kilometers, multiply by 1.6 . 
Table 2-8. Centralization at the Idaho National Engineering Laboratory cumulative shipment-miles for train shipments. ${ }^{2}$

\begin{tabular}{|c|c|c|c|c|}
\hline Route & Miles & Shipments & Shipment-miles & $\begin{array}{r}\text { Percent of Cumulative } \\
\text { Shipment-miles }\end{array}$ \\
\hline Military Ocean Terminal Sunny Point, NC & 2657.0 & 541 & $1.44 \mathrm{E}+06$ & 30.744 \\
\hline Gouvenour, NY & 2332.0 & 236 & $5.50 \mathrm{E}+05$ & 42.516 \\
\hline Gaithersburg, MD & 2335.0 & 188 & $4.39 \mathrm{E}+05$ & 51.905 \\
\hline Hanford Site & 658.0 & 646 & $4.25 \mathrm{E}+05$ & 60.996 \\
\hline Savannah River Site & 2407.0 & 173 & $4.16 \mathrm{E}+05$ & 69.902 \\
\hline NEL Oakland, CA & 1102.0 & 231 & $2.55 \mathrm{E}+05$ & 75.347 \\
\hline University of Missouri - Columbia & 1402.0 & 145 & $2.03 E+05$ & 79.695 \\
\hline Massachusetts Institute of Technology & 2559.0 & 69 & $1.77 \mathrm{E}+05$ & 83.472 \\
\hline INEL University of Michigan & 1823.0 & 72 & $1.31 \mathrm{E}+05$ & 86.279 \\
\hline Rhode Island Nuclear Science Center & 2620.0 & 32 & $8.38 \mathrm{E}+04$ & 88.072 \\
\hline INEL . Oak Ridge Reservation & 2055.0 & 26 & $5.34 \mathrm{E}+04$ & 89.215 \\
\hline INEL Georgia Institute Technology & 2186.0 & 19 & $4.15 E+04$ & 90.103 \\
\hline Rensselaer Polytechnic Institute & 2388.0 & 16 & $3.82 \mathrm{E}+04$ & 90.921 \\
\hline INEL Brookhaven National Laboratory & 2607.0 & 14 & $3.65 \mathrm{E}+04$ & 91.701 \\
\hline INEL $\quad$ Texas A\&M University & 1920.0 & 15 & $2.88 \mathrm{E}+04$ & 92.317 \\
\hline University of Virginia & 2357.0 & 12 & $2.83 \mathrm{E}+04$ & 92.922 \\
\hline INEL Cornell University & 2296.0 & 12 & $2.76 \mathrm{E}+04$ & 93.511 \\
\hline State University of New York - Buffalo & 2091.0 & 12 & $2.51 \mathrm{E}+04$ & 94.048 \\
\hline INEL Fort St. Vrain Nuclear Generating Station & 672.0 & 35 & $2.35 \mathrm{E}+04$ & 94.551 \\
\hline INEL North Carolina State University & 2626.0 & 8 & $2.10 \mathrm{E}+04$ & 95.001 \\
\hline University of Illinois & 1612.0 & 13 & $2.10 \mathrm{E}+04$ & 95.449 \\
\hline University of Florida & 2592.0 & 7 & $1.81 \mathrm{E}+04$ & 95.837 \\
\hline University of Wisconsin & 1664.0 & 9 & $1.50 \mathrm{E}+04$ & 96.157 \\
\hline Pennsylvania State University & 2214.0 & 6 & $1.33 \mathrm{E}+04$ & 96.441 \\
\hline INEL University of Maryland & 2354.0 & 5 & $1.18 \mathrm{E}+04$ & 96.693 \\
\hline INEL University of Missouri - Rolla & 1619.0 & 7 & $1.13 \mathrm{E}+04$ & 96.935 \\
\hline INEL University of Lowell & 2549.0 & 4 & $1.02 \mathrm{E}+04$ & 97.153 \\
\hline INEL Worcester Polytechnic Institute & 2544.0 & 4 & $1.02 \mathrm{E}+04$ & 97.371 \\
\hline San Diego, CA & 1076.0 & 8 & $8.61 \mathrm{E}+03$ & 97.555 \\
\hline INEL West Valley Demonstration Project & 2108.0 & 4 & $8.43 \mathrm{E}+03$ & 97.736 \\
\hline INEL Kansas State University & 1197.0 & 7 & $8.38 \mathrm{E}+03$ & 97.915 \\
\hline University of Texas & 1927.0 & 4 & $7.71 E+03$ & 98.080 \\
\hline INEL Manhattan College & 2524.0 & 3 & $7.57 E+03$ & 98.242 \\
\hline Sandia National Laboratories-Albuquerque & 1247.0 & 6 & $7.48 \mathrm{E}+03$ & 98.402 \\
\hline INEL $\quad$ Purdue University & 1813.0 & 4 & $7.25 \mathrm{E}+03$ & 98.557 \\
\hline INEL Washington State University & 876.0 & 8 & $7.01 E+03$ & 98.707 \\
\hline Midland, MI & 1961.0 & 3 & $5.88 \mathrm{E}+03$ & 98.832 \\
\hline INEL $\quad$ Ohio State University & 1936.0 & 3 & $5.81 \mathrm{E}+03$ & 98.957 \\
\hline INEL Oregon State University & 878.0 & 6 & $5.27 \mathrm{E}+03$ & 99.069 \\
\hline INEL $\quad$ Iowa State University & 1242.0 & 4 & $4.97 \mathrm{E}+03$ & 99.176 \\
\hline INEL Argonne National Laboratory - East & 1655.0 & 3 & $4.97 \mathrm{E}+03$ & 99.282 \\
\hline INEL Los Alamos National Laboratory & 1179.0 & 4 & $4.72 \mathrm{E}+03$ & 99.383 \\
\hline INEL $\quad$ Babcock \& Wilcox & 2333.0 & 2 & $4.67 \mathrm{E}+03$ & 99.482 \\
\hline INEL $\quad$ Denver, $\mathrm{CO}$ & 708.0 & 6 & $4.25 \mathrm{E}+03$ & 99.573 \\
\hline INEL University of Arizona & 1376.0 & 3 & $4.13 E+03$ & 99.662 \\
\hline INEL $\quad$ Pleasanton, CA & 965.0 & 4 & $3.86 \mathrm{E}+03$ & 99.744 \\
\hline INEL University of California - Irvine & 982.0 & 3 & $2.95 \mathrm{E}+03$ & 99.807 \\
\hline INEL $\quad$ San Ramon, CA & 965.0 & 3 & $2.90 \mathrm{E}+03$ & 99.869 \\
\hline INEL McClellan AFB, CA & 853.0 & 3 & $2.56 \mathrm{E}+03$ & 99.924 \\
\hline INEL $\quad$ Reed College & 785.0 & 2 & $1.57 \mathrm{E}+03$ & 99.957 \\
\hline INEL University of New Mexico & 1250.0 & 1 & $1.25 \mathrm{E}+03$ & 99.984 \\
\hline INEL University of Utah & 228.0 & 3 & $6.84 \mathrm{E}+02$ & 99.999 \\
\hline INEL Idaho State University & 56.0 & 1 & $5.60 \mathrm{E}+01$ & 100.000 \\
\hline Total & & 2655 & $4.68 \mathrm{E}+06$ & \\
\hline
\end{tabular}


Table 2-9. Centralization at the Oak Ridge Reservation cumulative shipment-miles for train shipments.

\begin{tabular}{|c|c|c|c|c|c|}
\hline & Route & Miles & Shipments & Shipment-miles & $\begin{array}{r}\text { Percent of Cumulative } \\
\text { Shipment-miles }\end{array}$ \\
\hline Oak Ridge Reservation & Hanford Site & 2601.0 & 646 & $1.68 \mathrm{E}+06$ & 37.080 \\
\hline Oak Ridge Reservation & Idaho National Engineering Laboratory & 2055.0 & 366 & $7.52 \mathrm{E}+05$ & 53.678 \\
\hline Oak Ridge Reservation & Portland, OR & 2827.0 & 231 & $6.53 \mathrm{E}+05$ & 68.089 \\
\hline Oak Ridge Reservation & Hampton Roads, VA & 689.0 & 541 & $3.73 \mathrm{E}+05$ & 76.315 \\
\hline Oak Ridge Reservation & Gouvenour, NY & 972.0 & 236 & $2.29 \mathrm{E}+05$ & 81.378 \\
\hline Oak Ridge Reservation & Gaithersburg, MD & 819.0 & 188 & $1.54 \mathrm{E}+05$ & 84.775 \\
\hline Oak Ridge Reservation & University of Missouri - Columbia & 695.0 & 145 & $1.01 \mathrm{E}+05$ & 86.999 \\
\hline Oak Ridge Reservation & Massachusetts Institute of Technology & 1199.0 & 69 & $8.27 \mathrm{E}+04$ & 88.825 \\
\hline Oak Ridge Reservation & Savannah River Site & 417.0 & 173 & $7.21 \mathrm{E}+04$ & 90.417 \\
\hline Oak Ridge Reservation & Fort St. Vrain Nuclear Generating Station & 1526.0 & 35 & $5.34 \mathrm{E}+04$ & 91.596 \\
\hline Oak Ridge Reservation & University of Michigan & 591.0 & 72 & $4.26 \mathrm{E}+04$ & 92.535 \\
\hline Oak Ridge Reservation & Rhode Island Nuclear Science Center & 1259.0 & 32 & $4.03 E+04$ & 93.424 \\
\hline Oak Ridge Reservation & San Diego, $\mathrm{CA}$ & 2709.0 & 8 & $2.17 \mathrm{E}+04$ & 93.902 \\
\hline Oak Ridge Reservation & Washington State University & 2536.0 & 8 & $2.03 E+04$ & 94.350 \\
\hline Oak Ridge Reservation & Oregon State University & 3055.0 & 6 & $1.83 \mathrm{E}+04$ & 94.754 \\
\hline Oak Ridge Reservation & Rensselaer Polytechnic Institute & 1028.0 & 16 & $1.64 \mathrm{E}+04$ & 95.117 \\
\hline Oak Ridge Reservation & Brookhaven National Laboratory & 1152.0 & 14 & $1.61 \mathrm{E}+04$ & 95.473 \\
\hline Oak Ridge Reservation & Texas A\&M University & 1013.0 & 15 & $1.52 \mathrm{E}+04$ & 95.809 \\
\hline Oak Ridge Reservation & Pleasanton, CA & 3029.0 & 4 & $1.21 \mathrm{E}+04$ & 96.076 \\
\hline Oak Ridge Reservation & Cornell University & 935.0 & 12 & $1.12 E+04$ & 96.324 \\
\hline Oak Ridge Reservation & Sandia National Laboratory-Albuquerque & 1749.0 & 6 & $1.05 \mathrm{E}+04$ & 96.555 \\
\hline Oak Ridge Reservation & Denver, $\mathrm{CO}$ & 1560.0 & 6 & $9.36 \mathrm{E}+03$ & 96.762 \\
\hline Oak Ridge Reservation & San Ramon, CA & 3029.0 & 3 & $9.09 \mathrm{E}+03$ & 96.962 \\
\hline Oak Ridge Reservation & State University of New York - Buffalo & 731.0 & 12 & $8.77 E+03$ & 97.156 \\
\hline Oak Ridge Reservation & McClellan AFB, CA & 2747.0 & 3 & $8.24 \mathrm{E}+03$ & 97.338 \\
\hline Oak Ridge Reservation & University of California - Irvine & 2615.0 & 3 & $7.85 \mathrm{E}+03$ & 97.511 \\
\hline Oak Ridge Reservation & University of Illinois & 592.0 & 13 & $7.70 \mathrm{E}+03$ & 97.681 \\
\hline Oak Ridge Reservation & University of Wisconsin & 765.0 & 9 & $6.89 \mathrm{E}+03$ & 97.833 \\
\hline Oak Ridge Reservation & Los Alamos National Laboratory & 1686.0 & 4 & $6.74 E+03$ & 97.981 \\
\hline Oak Ridge Reservation & Kansas State University & 948.0 & 7 & $6.64 E+03$ & 98.128 \\
\hline Oak Ridge Reservation & University of Arizona & 2103.0 & 3 & $6.31 E+03$ & 98.267 \\
\hline Oak Ridge Reservation & University of Utah & 2051.0 & 3 & $6.15 E+03$ & 98.403 \\
\hline Oak Ridge Reservation & Reed College & 2827.0 & 2 & $5.65 \mathrm{E}+03$ & 98.528 \\
\hline Oak Ridge Reservation & University of Virginia & 451.0 & 12 & $5.41 E+03$ & 98.647 \\
\hline Oak Ridge Reservation & Pennsylvania State University & 822.0 & 6 & $4.93 E+03$ & 98.756 \\
\hline Oak Ridge Reservation & University of Lowell & 1189.0 & 4 & $4.76 \mathrm{E}+03$ & 98.861 \\
\hline Oak Ridge Reservation & Worcester Polytechnic Institute & 1183.0 & 4 & $4.73 \mathrm{E}+03$ & 98.965 \\
\hline Oak Ridge Reservation & University of Missouri - Rolla & 640.0 & 7 & $4.48 \mathrm{E}+03$ & 99.064 \\
\hline Oak Ridge Reservation & University of Florida & 634.0 & 7 & $4.44 \mathrm{E}+03$ & 99.162 \\
\hline Oak Ridge Reservation & Georgia Institute of Technology & 228.0 & 19 & 4.33E+03 & 99.258 \\
\hline Oak Ridge Reservation & University of Texas & 1045.0 & 4 & $4.18 \mathrm{E}+03$ & 99.350 \\
\hline Oak Ridge Reservation & North Carolina State University & 511.0 & 8 & $4.09 \mathrm{E}+03$ & 99.440 \\
\hline Oak Ridge Reservation & Iowa State University & 954.0 & 4 & $3.82 \mathrm{E}+03$ & 99.524 \\
\hline Oak Ridge Reservation & West Valley Demonstration Project & 889.0 & 4 & $3.56 \mathrm{E}+03$ & 99.603 \\
\hline Oak Ridge Reservation & Manhattan College & 1164.0 & 3 & $3.49 \mathrm{E}+03$ & 99.680 \\
\hline Oak Ridge Reservation & University of Maryland & 582.0 & 5 & $2.91 \mathrm{E}+03$ & 99.744 \\
\hline Oak Ridge Reservation & Idaho State University & 1996.0 & 1 & $2.00 \mathrm{E}+03$ & 99.788 \\
\hline Oak Ridge Reservation & Purdue University & 495.0 & 4 & $1.98 \mathrm{E}+03$ & 99.832 \\
\hline Oak Ridge Reservation & Argonne National Laboratory - East & 648.0 & 3 & $1.94 \mathrm{E}+03$ & 99.875 \\
\hline Oak Ridge Reservation & Midland, MI & 645.0 & 3 & $1.94 \mathrm{E}+03$ & 99.917 \\
\hline Oak Ridge Reservation & University of New Mexico & 1749.0 & 1 & $1.75 \mathrm{E}+03$ & 99.956 \\
\hline Oak Ridge Reservation & Ohio State University & 406.0 & 3 & $1.22 \mathrm{E}+03$ & 99.983 \\
\hline Oak Ridge Reservation & Babcock \& Wilcox & 386.0 & 2 & $7.72 \mathrm{E}+02$ & 100.000 \\
\hline Total & & & 2995 & $4.53 \mathrm{E}+06$ & \\
\hline
\end{tabular}


Table 2-10. Centralization at the Nevada Test Site cumulative shipment-miles for train shipments. ${ }^{2}$

\begin{tabular}{|c|c|c|c|c|c|}
\hline & Route & Miles & Shipments & Shipment-miles & $\begin{array}{l}\text { Percent of Cumulative } \\
\text { Shipment-miles }\end{array}$ \\
\hline Nevada Test Site & Military Ocean Terminal Sunny Point, NC & 3089.0 & 541 & $1.67 \mathrm{E}+06$ & 26.834 \\
\hline Nevada Test Site & Hanford Site & 1302.0 & 646 & $8.41 \mathrm{E}+05$ & 40.340 \\
\hline Nevada Test Site & Gouvenour, NY & 2763.0 & 236 & $6.52 \mathrm{E}+05$ & 50.810 \\
\hline Nevada Test Site & Gaithersburg, MD & 2767.0 & 188 & $5.20 \mathrm{E}+05$ & 59.163 \\
\hline Nevada Test Site & Savannah River Site & 2839.0 & 173 & $4.91 \mathrm{E}+05$ & 67.050 \\
\hline Nevada Test Site & Seattle, WA & 1620.0 & 231 & $3.74 \mathrm{E}+05$ & 73.059 \\
\hline Nevada Test Site & Idaho National Engineering Laboratory & 756.0 & 366 & $2.77 \mathrm{E}+05$ & 77.502 \\
\hline Nevada Test Site & University of Missouri - Columbia & 1833.0 & 145 & $2.66 \mathrm{E}+05$ & 81.770 \\
\hline Nevada Test Site & Massachusetts Institute of Technology & 2990.0 & 69 & $2.06 \mathrm{E}+05$ & 85.082 \\
\hline Nevada Test Site & University of Michigan & 2255.0 & 72 & $1.62 E+05$ & 87.689 \\
\hline Nevada Test Site & Rhode Island Nuclear Science Center & 3051.0 & 32 & $9.76 \mathrm{E}+04$ & 89.257 \\
\hline Nevada Test Site & Oak Ridge Reservation & 2487.0 & 26 & $6.47 \mathrm{E}+04$ & 90.295 \\
\hline Nevada Test Site & Georgia Institute of Technology & 2618.0 & 19 & $4.97 \mathrm{E}+04$ & 91.094 \\
\hline Nevada Test Site & Rensselaer Polytechnic Institute & 2820.0 & 16 & $4.51 E+04$ & 91.819 \\
\hline Nevada Test Site & Brookhaven National Laboratory & 3039.0 & 14 & $4.25 \mathrm{E}+04$ & 92.502 \\
\hline Nevada Test Site & Fort St. Vrain Nuclear Generating Station & 1104.0 & 35 & $3.86 \mathrm{E}+04$ & 93.122 \\
\hline Nevada Test Site & University of Virginia & 2788.0 & 12 & $3.35 \mathrm{E}+04$ & 93.660 \\
\hline Nevada Test Site & Cornell University & 2727.0 & 12 & $3.27 \mathrm{E}+04$ & 94.185 \\
\hline Nevada Test Site & State University of New York - Buffalo & 2522.0 & 12 & $3.03 E+04$ & 94.671 \\
\hline Nevada Test Site & Texas A\&M University & 1967.0 & 15 & $2.95 \mathrm{E}+04$ & 95.145 \\
\hline Nevada Test Site & University of Illinois & 2044.0 & 13 & $2.66 \mathrm{E}+04$ & 95.571 \\
\hline Nevada Test Site & North Carolina State University & 3058.0 & 8 & $2.45 \mathrm{E}+04$ & 95.964 \\
\hline Nevada Test Site & University of Florida & 3024.0 & 7 & $2.12 \mathrm{E}+04$ & 96.304 \\
\hline Nevada Test Site & University of Wisconsin & 2096.0 & 9 & $1.89 \mathrm{E}+04$ & 96.607 \\
\hline Nevada Test Site & Pennsylvania State University & 2646.0 & 6 & $1.59 \mathrm{E}+04$ & 96.862 \\
\hline Nevada Test Site & University of Missouri - Rolla & 2050.0 & 7 & $1.44 \mathrm{E}+04$ & 97.092 \\
\hline Nevada Test Site & University of Maryland & 2786.0 & 5 & $1.39 \mathrm{E}+04$ & 97.316 \\
\hline Nevada Test Site & Washington State University & 1520.0 & 8 & $1.22 \mathrm{E}+04$ & 97.511 \\
\hline Nevada Test Site & University of Lowell & 2980.0 & 4 & $1.19 \mathrm{E}+04$ & 97.703 \\
\hline Nevada Test Site & Worcester Polytechnic Institute & 2975.0 & 4 & $1.19 \mathrm{E}+04$ & 97.894 \\
\hline Nevada Test Site & Kansas State University & 1628.0 & 7 & $1.14 \mathrm{E}+04$ & 98.077 \\
\hline Nevada Test Site & West Valley Demonstration Project & 2554.0 & 4 & $1.02 \mathrm{E}+04$ & 98.241 \\
\hline Nevada Test Site & University of Texas & 2358.0 & 4 & $9.43 \mathrm{E}+03$ & 98.392 \\
\hline Nevada Test Site & Purdue University & 2245.0 & 4 & $8.98 \mathrm{E}+03$ & 98.536 \\
\hline Nevada Test Site & Manhattan College & 2956.0 & 3 & $8.87 \mathrm{E}+03$ & 98.679 \\
\hline Nevada Test Site & Oregon State University & 1400.0 & 6 & $8.40 \mathrm{E}+03$ & 98.814 \\
\hline Nevada Test Site & Midland, MI & 2392.0 & 3 & $7.18 \mathrm{E}+03$ & 98.929 \\
\hline Nevada Test Site & Ohio State University & 2367.0 & 3 & $7.10 \mathrm{E}+03$ & 99.043 \\
\hline Nevada Test Site & Argonne National Laboratory - East & 2348.0 & 3 & $7.04 \mathrm{E}+03$ & 99.156 \\
\hline Nevada Test Site & Denver, $\mathrm{CO}$ & 1140.0 & 6 & $6.84 \mathrm{E}+03$ & 99.266 \\
\hline Nevada Test Site & Iowa State University & 1674.0 & 4 & $6.70 \mathrm{E}+03$ & 99.373 \\
\hline Nevada Test Site & Sandia National Laboratory-Albuquerque & 1065.0 & 6 & $6.39 \mathrm{E}+03$ & 99.476 \\
\hline Nevada Test Site & Babcock \& Wilcox & 2765.0 & 2 & $5.53 \mathrm{E}+03$ & 99.565 \\
\hline Nevada Test Site & Los Alamos National Laboratory & 1169.0 & 4 & $4.68 \mathrm{E}+03$ & 99.640 \\
\hline Nevada Test Site & San Diego, $\mathrm{CA}$ & 518.0 & 8 & $4.14 \mathrm{E}+03$ & 99.706 \\
\hline Nevada Test Site & Pleasanton, $\mathrm{CA}$ & 838.0 & 4 & $3.35 \mathrm{E}+03$ & 99.760 \\
\hline Nevada Test Site & Reed College & 1429.0 & 2 & $2.86 \mathrm{E}+03$ & 99.806 \\
\hline Nevada Test Site & San Ramon, CA & 838.0 & 3 & $2.51 \mathrm{E}+03$ & 99.847 \\
\hline Nevada Test Site & McClellan AFB, CA & 827.0 & 3 & $2.48 \mathrm{E}+03$ & 99.886 \\
\hline Nevada Test Site & University of Arizona & 818.0 & 3 & $2.45 \mathrm{E}+03$ & 99.926 \\
\hline Nevada Test Site & University of Utah & 528.0 & 3 & $1.58 \mathrm{E}+03$ & 99.951 \\
\hline Nevada Test Site & University of Califormia - Irvine & 424.0 & 3 & $1.27 \mathrm{E}+03$ & 99.972 \\
\hline Nevada Test Site & University of New Mexico & 1065.0 & 1 & $1.07 \mathrm{E}+03$ & 99.989 \\
\hline Nevada Test Site & Idaho State University & 700.0 & 1 & $7.00 \mathrm{E}+02$ & 100.00 \\
\hline Total & & & 3021 & $6.23 E+06$ & \\
\hline
\end{tabular}


Table 2-11. Comparison of HIGHWAY mileage data with Map-n-Go mileage data.

\begin{tabular}{|c|c|c|c|c|c|c|}
\hline Centralization Alternative & $\begin{array}{r}\text { Origin } \\
\end{array}$ & $\begin{array}{r}\text { (P) } \\
\text { HIGHWAY Distance } \\
\text { (miles) }\end{array}$ & $\begin{array}{r}(0) \\
\text { Map-n-Go Distance } \\
\text { (miles) }\end{array}$ & Difference & $\begin{array}{r}\text { Absolute } \\
\text { Value of } \\
\text { Percent } \\
\text { Difference } \\
\end{array}$ & {$[(\mathrm{P}-\mathrm{O}) / \mathrm{P}]^{2}$} \\
\hline Hanford Site & Savannah River Site & 2727.0 & 2735.0 & -8 & 0.29 & $8.61 \mathrm{E}-06$ \\
\hline Hanford Site & Hampton Roads, VA & 2903.0 & 2942.0 & -39 & 1.34 & $1.80 \mathrm{E}-04$ \\
\hline Hanford Site & INEL & 599.0 & 623.0 & -24 & 4.01 & $1.61 \mathrm{E}-03$ \\
\hline Hanford Site & Alexandria Bay, NY & 2768.0 & 2655.0 & 113 & 4.08 & $1.67 \mathrm{E}-03$ \\
\hline Hanford Site & Gaithersburg, MD & 2732.0 & 2728.0 & 4 & 0.15 & $2.14 \mathrm{E}-06$ \\
\hline Hanford Site & Oak Ridge Reservation & 2464.0 & 2484.0 & -20 & 0.81 & $6.59 \mathrm{E}-05$ \\
\hline Hanford Site & Fort St. Vrain Nuclear Generating Station & 1108.0 & 1141.0 & -33 & 2.98 & $8.87 \mathrm{E}-04$ \\
\hline Hanford Site & University of Missouri - Columbia & 1870.0 & 1880.0 & -10 & 0.53 & $2.86 \mathrm{E}-05$ \\
\hline Savannah River Site & Hanford Site & 2727.0 & 2735.0 & -8 & 0.29 & 8.61E-06 \\
\hline Savannah River Site & INEL & 2311.0 & 2267.0 & 44 & 1.90 & $3.62 \mathrm{E}-04$ \\
\hline Savannah River Site & Seattle, WA & 2900.0 & 2876.0 & 24 & 0.83 & $6.85 \mathrm{E}-05$ \\
\hline Idaho National Engineering Laboratory & Savannah River Site & 2311.0 & 2267.0 & 44 & 1.90 & $3.62 \mathrm{E}-04$ \\
\hline Idaho National Engineering Laboratory & Hampton Roads, VA & 2487.0 & 2509.0 & -22 & 0.88 & 7.83E-05 \\
\hline Idaho National Engineering Laboratory & Hanford Site & 599.0 & 623.0 & -24 & 4.01 & $1.61 \mathrm{E}-03$ \\
\hline Idaho National Engineering Laboratory & Alexandria Bay, NY & 2352.0 & 2232.0 & 120 & 5.10 & $2.60 \mathrm{E}-03$ \\
\hline Idaho National Engineering Laboratory & Gaithersburg, MD & 2316.0 & 2283.0 & 33 & 1.42 & $2.03 \mathrm{E}-04$ \\
\hline Idaho National Engineering Laboratory & Oak Ridge Reservation & 2048.0 & 2004.0 & 44 & 2.15 & $4.62 \mathrm{E}-04$ \\
\hline Idaho National Engineering Laboratory & Oakland, $\mathrm{CA}$ & 963.0 & 941.0 & 22 & 2.28 & $5.22 \mathrm{E}-04$ \\
\hline Idaho National Engineering Laboratory & University of Missouri - Columbia & 1454.0 & 1411.0 & 43 & 2.96 & $8.75 \mathrm{E}-04$ \\
\hline Oak Ridge Reservation & Hanford Site & 2464.0 & 2484.0 & -20 & 0.81 & $6.59 \mathrm{E}-05$ \\
\hline Oak Ridge Reservation & INEL & 2048.0 & 2004.0 & 44 & 2.15 & $4.62 \mathrm{E}-04$ \\
\hline Oak Ridge Reservation & Seattle, WA & 2636.0 & 2613.0 & 23 & 0.87 & $7.61 \mathrm{E}-05$ \\
\hline Nevada Test Site & Savannah River Site & 2414.0 & 2383.0 & 31 & 1.28 & $1.65 \mathrm{E}-04$ \\
\hline Nevada Test Site & Hanford Site & 1128.0 & 1144.0 & -16 & 1.42 & $2.01 \mathrm{E}-04$ \\
\hline Nevada Test Site & Hampton Roads, VA & 2590.0 & 2721.0 & -131 & 5.06 & $2.56 \mathrm{E}-03$ \\
\hline Nevada Test Site & INEL & 712.0 & 688.0 & 24 & 3.37 & $1.14 \mathrm{E}-03$ \\
\hline Nevada Test Site & Alexandria Bay, NY & 2619.0 & 2503.0 & 116 & 4.43 & $1.96 \mathrm{E}-03$ \\
\hline Nevada Test Site & Gaithersburg, MD & 2488.0 & 2507.0 & -19 & 0.76 & $5.83 \mathrm{E}-05$ \\
\hline Nevada Test Site & Seattle, WA & 1322.0 & 1304.0 & 18 & 1.36 & $1.85 \mathrm{E}-04$ \\
\hline Nevada Test Site & Oak Ridge Reservation & 2151.0 & 2120.0 & 31 & 1.44 & $2.08 \mathrm{E}-04$ \\
\hline Maximum & & & & 120.0 & 5.10 & \\
\hline Minimum & & & & -131.0 & 0.15 & \\
\hline Mean & & & & 13.5 & 2.03 & \\
\hline Relative Root Mean Square Error & & & & & & $2.49 \mathrm{E}-02$ \\
\hline
\end{tabular}

a. To convert from miles to kilometers, multiply by 1.6 . 
Table 2-12. Comparison of INTERLINE mileage data with mileage data from the Railroad Information Service. ${ }^{a}$

\begin{tabular}{|c|c|c|c|c|c|c|}
\hline Centralization Alternative & Origin & $\begin{array}{r}(\mathbf{P}) \\
\text { INTERLINE Distance } \\
\text { (miles) }\end{array}$ & 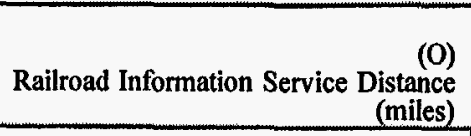 & Difference & $\begin{array}{r}\text { Absolute } \\
\text { Value of } \\
\text { Percent } \\
\text { Difference } \\
\end{array}$ & {$[(\mathbf{P}-\mathbf{O}) / \mathbf{P}]^{2}$} \\
\hline Hanford Site & Military Ocean Terminal Sunny Point, NC & 3127.0 & 3139.0 & -12 & 0.38 & $1.47 \mathrm{E}-05$ \\
\hline Hanford Site & Gouvenour, NY & 2784.0 & 2802.0 & -18 & 0.65 & $4.18 \mathrm{E}-05$ \\
\hline Hanford Site & Gaithersburg, MD & 2787.0 & 2811.0 & -24 & 0.86 & $7.42 \mathrm{E}-05$ \\
\hline Hanford Site & Savannah River Site & 2945.0 & 2961.0 & -16 & 0.54 & $2.95 \mathrm{E}-05$ \\
\hline Hanford Site & University of Missouri - Columbia & 2044.0 & 2020.0 & 24 & 1.17 & $1.38 \mathrm{E}-04$ \\
\hline Hanford Site & INEL & 675.0 & 670.0 & 5 & 0.74 & $5.49 \mathrm{E}-05$ \\
\hline Hanford Site & Oakland, CA & 860.0 & 864.0 & -4 & 0.47 & $2.16 \mathrm{E}-05$ \\
\hline Hanford Site & Massachusetts Institute of Technology & 3006.0 & 3040.0 & -34 & 1.13 & $1.28 \mathrm{E}-04$ \\
\hline Savannah River Site & Hanford Site & 2944.0 & 2961.0 & -17 & 0.58 & $3.33 \mathrm{E}-05$ \\
\hline Savannah River Site & INEL & 2384.0 & 2386.0 & -2 & 0.08 & $7.04 \mathrm{E}-07$ \\
\hline Savannah River Site & Oakland, CA & 3180.0 & 3196.0 & -16 & 0.50 & $2.53 \mathrm{E}-05$ \\
\hline Savannah River Site & Gouvenour, NY & 1280.0 & 1280.0 & 0 & 0.00 & 0.00 \\
\hline Savannah River Site & Hampton Roads, VA & 604.0 & 603.0 & 1 & 0.17 & $2.74 \mathrm{E}-06$ \\
\hline Idaho National Engineering Laboratory & Military Ocean Terminal Sunny Point, NC & 2621.0 & 2606.0 & 15 & 0.57 & $3.28 \mathrm{E}-05$ \\
\hline Idaho National Engineering Laboratory & Gouvenour, NY & 2472.0 & 2477.0 & -5 & 0.20 & $4.09 \mathrm{E}-06$ \\
\hline Idaho National Engineering Laboratory & Gaithersburg, MD & 2547.0 & 2539.0 & 8 & 0.31 & $9.87 \mathrm{E}-06$ \\
\hline Idaho National Engineering Laboratory & Hanford Site & 675.0 & 670.0 & 5 & 0.74 & $5.49 \mathrm{E}-05$ \\
\hline Idaho National Engineering Laboratory & Savannah River Site & 2362.0 & 2386.0 & -24 & 1.02 & $1.03 \mathrm{E}-04$ \\
\hline Idaho National Engineering Laboratory & Oakland, CA & 1139.0 & 1147.0 & -8 & 0.70 & $4.93 \mathrm{E}-05$ \\
\hline Idaho National Engineering Laboratory & University of Missouri - Columbia & 1404.0 & 1411.0 & .7 & 0.50 & $2.49 \mathrm{E}-05$ \\
\hline Idaho National Engineering Laboratory & Massachusetts Institute of Technology & 2699.0 & 2718.0 & -19 & 0.70 & $4.96 \mathrm{E}-05$ \\
\hline Oak Ridge Reservation & Hanford Site & 2647.0 & 2686.0 & -39 & 1.47 & $2.17 \mathrm{E}-04$ \\
\hline Oak Ridge Reservation & INEL & 2111.0 & 2142.0 & -31 & 1.47 & $2.16 \mathrm{E}-04$ \\
\hline Oak Ridge Reservation & Portland, OR & 2839.0 & 2860.0 & -21 & 0.74 & $5.47 \mathrm{E}-05$ \\
\hline Oak Ridge Reservation & Hampton Roads, VA & 901.0 & 904.0 & -3 & 0.33 & $1.11 \mathrm{E}-05$ \\
\hline Oak Ridge Reservation & Gouvenour, NY & 950.0 & 968.0 & -18 & 1.89 & $3.59 \mathrm{E}-04$ \\
\hline Nevada Test Site & Military Ocean Terminal Sunny Point, NC & 2973.0 & 2968.0 & 5 & 0.17 & $2.83 \mathrm{E}-06$ \\
\hline Nevada Test Site & Hanford Site & 1235.0 & 1234.0 & 1 & 0.08 & $6.56 \mathrm{E}-07$ \\
\hline Nevada Test Site & Gouvenour, NY & 2824.0 & 2839.0 & -15 & 0.53 & $2.82 \mathrm{E}-05$ \\
\hline Nevada Test Site & Gaithersburg, MD & 2899.0 & 2901.0 & -2 & 0.07 & $4.76 \mathrm{E}-07$ \\
\hline Nevada Test Site & Savannah River Site & 2741.0 & 2748.0 & -7 & 0.26 & $6.52 \mathrm{E}-06$ \\
\hline Nevada Test Site & Seattle, WA & 1532.0 & 1529.0 & 3 & 0.20 & $3.83 \mathrm{E}-06$ \\
\hline Nevada Test Site & INEL & 672.0 & 676.0 & -4 & 0.60 & $3.54 \mathrm{E}-05$ \\
\hline Nevada Test Site & University of Missouri - Columbia & 1761.0 & 1773.0 & -12 & 0.68 & $4.64 \mathrm{E}-05$ \\
\hline Maximum & & & & 24.0 & 1.89 & \\
\hline Minimum & & & & -39.0 & 0.0 & \\
\hline Mean & & & & -8.6 & 0.60 & \\
\hline Relative Root Mean Square Error & & & & & & $7.43 \mathrm{E}-03$ \\
\hline
\end{tabular}




\section{VALIDATION OF THE INCIDENT-FREE UNIT RISK FACTORS CALCULATED USING THE RADTRAN 4 COMPUTER CODE}

\subsection{Validation Analysis}

The RADTRAN 4 computer code (Neuhauser and Kanipe 1995) was used to estimate incident-free unit risk factors for the transportation analyses in (DOE 1995). Unit risk factors provide estimates of the radiation dose to groups of people from transporting a shipment of radioactive material over a unit distance of travel in a population density zone (rural, suburban, and urban). Unit risk factors have units of collective dose (person-rem).

The purpose of this analysis was to validate the incident-free unit risk factors for spent nuclear fuel shipments calculated using the RADTRAN 4 computer code by comparing them to hand-calculated values using identical data and equations. The definition of validation used in this analysis was "the test and evaluation of the completed software to ensure compliance with software requirements" (ASME 1989). In the context of this analysis, compliance with software requirements means that the differences between the unit risk factors calculated by RADTRAN 4 and the hand-calculated unit risk factors should be small (i.e., within the acceptance criterion established for the validation).

The acceptance criterion for this validation was a percent difference of 5 percent. If the RADTRAN 4 calculated unit risk factor differed from the hand-calculated unit risk factor by more than 5 percent, then the result was considered unacceptable. If the RADTRAN 4 calculated unit risk factor differed from the hand-calculated unit risk factor by less than 5 percent, then the result was considered acceptable.

Radiation doses were estimated for two groups of people during incident-free transportation of radioactive material, the crew and the general population. For truck shipments, the crew were the drivers of the vehicle. For rail shipments, the crew were workers in close proximity to the shipping containers during inspection or classification of railcars. The general population was persons within $800 \mathrm{~m}$ ( $0.5 \mathrm{mile}$ ) of the road (off-link), persons sharing the road (on-link), and persons at stops. For rail shipments, there is also a nonlinear component to the radiation dose to the crew and persons at stops from inspections that occur at the beginning and end of the shipment. The unit risk factors 
estimated using the RADTRAN 4 computer code are listed in Table 3-1. These unit risk factors were estimated using a radiation dose rate of $14 \mathrm{mrem} / \mathrm{hr}$ at $1 \mathrm{~m} \mathrm{(3.25} \mathrm{feet)} \mathrm{from} \mathrm{the} \mathrm{shipping} \mathrm{container} \mathrm{and}$ assumed 100 percent gamma radiation. Appendix A contains the RADTRAN 4 output for these unit risk factors.

The equations used to calculate the unit risk factors are listed in Chapter 4 of the RADTRAN 4 Technical Manual (Neuhauser and Kanipe 1995). The unit risk factors estimated using hand calculations are listed in Table 3-2. Sections 3.4 and 3.5 contain the detailed hand calculations used to estimate the unit risk factors. Table 3-3 contains the percent differences between the RADTRAN 4 and the hand-calculated unit risk factors.

\subsection{Results of Validation Analysis}

The minimum percent difference observed in Table 3-3 was zero, which meant that RADTRAN 4 and the hand calculation yielded identical results. The maximum percent difference observed in Table 3-3 was 1.2 percent, well within the 5 percent validation criterion. These results show that the differences between the unit risk factors estimated by RADTRAN 4 and the unit risk factors estimated using hand calculations are small and are within the acceptance criterion established

Table 3-1. Unit risk factors calculated using the RADTRAN 4 computer code.

\begin{tabular}{lllll}
\hline & & \multicolumn{3}{c}{ Unit risk factors (person-rem) } \\
\cline { 3 - 5 } Mode & Exposure group & Rural & Suburban & Urban \\
\hline Truck & Crew & & & \\
& Off-link & $4.56 \mathrm{E}-5$ & $1.00 \mathrm{E}-4$ & $1.67 \mathrm{E}-4$ \\
& On-link & $1.23 \mathrm{E}-7$ & $1.63 \mathrm{E}-5$ & $1.08 \mathrm{E}-4$ \\
& Stops & $5.03 \mathrm{E}-6$ & $1.45 \mathrm{E}-5$ & $1.50 \mathrm{E}-4$ \\
& & $1.20 \mathrm{E}-4$ & $1.20 \mathrm{E}-4$ & $1.20 \mathrm{E}-4$ \\
Rail & & & \\
& Crew & $1.01 \mathrm{E}-5$ & $1.01 \mathrm{E}-5$ & $1.01 \mathrm{E}-5$ \\
& Nonlinear crew & $1.12 \mathrm{E}-2$ & $1.12 \mathrm{E}-2$ & $1.12 \mathrm{E}-2$ \\
& Off-link & $1.70 \mathrm{E}-7$ & $3.25 \mathrm{E}-5$ & $2.91 \mathrm{E}-4$ \\
& On-link & $6.62 \mathrm{E}-8$ & $8.47 \mathrm{E}-7$ & $2.35 \mathrm{E}-6$ \\
& Stops & $4.78 \mathrm{E}-6$ & $4.78 \mathrm{E}-6$ & $4.78 \mathrm{E}-6$ \\
& Nonlinear stops & $8.68 \mathrm{E}-3$ & $8.68 \mathrm{E}-3$ & $8.68 \mathrm{E}-3$ \\
\hline
\end{tabular}


for the validation. Therefore, the unit risk factors generated using the RADTRAN 4 computer code are considered validated.

Table 3-2. Hand-calculated unit risk factors.

\begin{tabular}{lllll}
\hline & & \multicolumn{3}{c}{ Unit risk factors (person-rem) } \\
\cline { 3 - 5 } Mode & Exposure group & Rural & Suburban & Urban \\
\hline Truck & & & & \\
& Crew & $4.52 \mathrm{E}-5$ & $9.94 \mathrm{E}-5$ & $1.66 \mathrm{E}-4$ \\
& Off-link & $1.22 \mathrm{E}-7$ & $1.61 \mathrm{E}-5$ & $1.07 \mathrm{E}-4$ \\
& On-link & $5.04 \mathrm{E}-6$ & $1.45 \mathrm{E}-5$ & $1.50 \mathrm{E}-4$ \\
& Stops & $1.20 \mathrm{E}-4$ & $1.20 \mathrm{E}-4$ & $1.20 \mathrm{E}-4$ \\
Rail & & & & \\
& Crew & $1.01 \mathrm{E}-5$ & $1.01 \mathrm{E}-5$ & $1.01 \mathrm{E}-5$ \\
& Nonlinear crew & $1.12 \mathrm{E}-2$ & $1.12 \mathrm{E}-2$ & $1.12 \mathrm{E}-2$ \\
& Off-link & $1.68 \mathrm{E}-7$ & $3.22 \mathrm{E}-5$ & $2.88 \mathrm{E}-4$ \\
& On-link & $6.63 \mathrm{E}-8$ & $8.48 \mathrm{E}-7$ & $2.35 \mathrm{E}-6$ \\
& Stops & $4.81 \mathrm{E}-6$ & $4.81 \mathrm{E}-6$ & $4.81 \mathrm{E}-6$ \\
& Nonlinear stops & $8.75 \mathrm{E}-3$ & $8.75 \mathrm{E}-3$ & $8.75 \mathrm{E}-3$ \\
\hline
\end{tabular}

Table 3-3. Percent differences between RADTRAN 4- and hand-calculated unit risk factors.

\begin{tabular}{lllll}
\hline & & \multicolumn{3}{c}{ Percent differences } \\
\cline { 4 - 5 } Mode & Exposure group & Rural & Suburban & Urban \\
\hline Truck & Crew & 0.88 & 0.60 & 0.60 \\
& Off-link & 0.82 & 1.2 & 0.93 \\
& On-link & 0.20 & 0.0 & 0.0 \\
& Stops & 0.0 & 0.0 & 0.0 \\
& & & & \\
& Lail & 0.0 & 0.0 & 0.0 \\
& Nonlinear crew & 0.0 & 0.0 & 0.0 \\
& Off-link & 1.2 & 0.93 & 1.0 \\
& On-link & -0.15 & -0.12 & 0.0 \\
& Linear stops & -0.62 & -0.62 & -0.62 \\
& Nonlinear stops & -0.80 & -0.80 & -0.80 \\
\hline
\end{tabular}




\subsection{Critiques of RADTRAN}

As part of this validation analysis, the following documents were examined for specific comments on the incident-free conceptual models used in RADTRAN:

- $\quad$ Public comments contained in Volume II of the Final Environmental Impact Statement on the Transportation of Radioactive Materials By Air and Other Modes (NRC 1977a)

- The Latest Nuclear Dilemma: Waste Shipment Peril Explored (Resnikoff et al. 1982)

- Analysis of Recent Council on Economic Priorities Newsletter (Jefferson et al. 1982)

- $\quad$ Transporting Spent Nuclear Fuel Allegations and Responses (Jefferson 1983)

- The Next Nuclear Gamble: Transportation and Storage of Nuclear Waste (Resnikoff 1983)

- Probabilistic Risk Assessment and Nuclear Waste Transportation: A Case Study of the Use of RADTRAN in the 1986 Environmental Assessment for Yucca Mountain (Resnikoff 1990)

- A Comparison of RISKIND and RADTRAN 4 (Brumburgh and Alesso 1993)

Based on these documents, six specific comments on the incident-free conceptual models used in RADTRAN were identified (some of these comments do not apply to the current version of RADTRAN, but do apply to previous versions of RADTRAN):

1. Radiation doses are underestimated because RADTRAN III does not include neutron doses.

2. Radiation doses are underestimated because RADTRAN does not account for skyscatter and groundscatter. Skyscatter and groundscatter can increase radiation doses 
by up to 25 percent, with groundscatter being much more significant because of the difference in density between the air and the ground.

3. Radiation doses are underestimated because RADTRAN uses a point-source configuration rather than a line-source or plane-source configuration for calculating radiation doses to the crew and persons sharing the transport link. In addition, a reference or justification is not provided for the method used to adjust the package size.

4. The values of $\mathrm{V}_{\mathrm{r}} / 2, \mathrm{~V}_{\mathrm{s}} / 2$, and $\mathrm{V}_{\mathrm{u}} / 2$ are not justified for urban or suburban areas.

5. No reference or derivation is provided to support the equation:

$$
I(x)=\int_{r=x}^{r=\infty} \frac{\exp (-\mu r) \cdot B(r)}{r\left(r^{2}-x^{2}\right)^{1 / 2}} d r
$$

These comments were evaluated for their applicability to RADTRAN 4. Responses to the comments are listed below:

1. Incident-free neutron doses have been incorporated in RADTRAN 4.

2. RADTRAN 4 does not account for skyscatter and groundscatter. To compensate for this, radiation doses in DOE (1995) were estimated using shielding factors of 1.0 for rural, suburban, and urban zones. For the population, the U.S. Nuclear Regulatory Commission recommends a time-weighted shielding factor of 0.50 (NRC 1977b). For people in cars, shielding factors range from 0.25 to 0.30 (Lauridsen and Hedemann-Jensen 1983). If the incident-free radiation doses were increased by 25 percent to account for skyscatter and groundscatter, this increase would be offset by the use of the shielding factors for the population and people in cars. In addition, the off-link radiation doses calculated using RADTRAN 4 were compared to the off-link radiation doses calculated using the RISKIND computer code (Yuan et al. 1993).

RADTRAN 4 does not account for the processes of attenuation, build-up, or groundscatter; RISKIND accounts for the processes of attenuation, build-up, and 
groundscatter. The process of attenuation decreases radiation doses; the processes of build-up and groundscatter increase radiation doses. RADTRAN 4 was found to overestimate off-link radiation doses by a factor of 1.5 to 2.3 when compared to RISKIND. Similar results were also obtained by Weiner and Neuhauser (1992), who found that the radiation doses calculated using RADTRAN 4 were overestimated when compared to radiation doses that were calculated accounting for attenuation, build-up, and groundscatter.

3. RADTRAN 4 uses the package shape factor, $k_{0}$, to convert a line source to an equivalent point source. The radiation dose rate for the crew calculated using RADTRAN 4 was compared to the radiation dose rate for the crew calculated using the RISKIND computer code, which accounts for the length and radius of the shipping container. RADTRAN 4 yielded a radiation dose rate of $9.1 \mathrm{mrem} / \mathrm{hr}$ for the crew, RISKIND yielded a radiation dose rate of $3.9 \mathrm{mrem} / \mathrm{hr}$; therefore, the approach used by RADTRAN 4 overestimates radiation doses.

4. The values of $V_{\mathrm{r}} / 2, \mathrm{~V}_{\mathrm{s}} / 2$, and $\mathrm{V}_{\mathrm{u}} / 2$ are intended to represent the average speed during rush hour; as such, they are considered to be reasonable estimates.

5. The derivation for this equation is presented in Yuan et al. (1993). 


\subsection{Detailed Hand Calculations for Incident-Free Truck Unit Risk Factors}

This section presents the detailed hand calculations for the incident-free truck crew dose, off-link dose, on-link dose, and dose at stops.

\subsubsection{List of Symbols - Truck}

\begin{tabular}{|c|c|c|}
\hline $\mathrm{DR}_{\mathrm{p}}$ & $=$ & Package dose rate $(14 \mathrm{mrem} / \mathrm{hr})$ \\
\hline DIST & $=$ & Distance $(1 \mathrm{~km})$ \\
\hline$f_{c s}$ & $=$ & Fraction of travel on city streets in urban zone $(0.050)$ \\
\hline$f_{\text {fwy }}$ & $=$ & Fraction of travel on freeways in rural and suburban zones (1.0) \\
\hline$f_{\text {rh }}$ & $=$ & Fraction of travel during rush hour in rural and suburban zones $(0.10)$ \\
\hline $\mathbf{k}_{0}$ & $=$ & Package shape factor $\left(6.25 \mathrm{~m}^{2}\right)$ \\
\hline $\mathbf{N}_{\mathrm{c}}$ & $=$ & Number of people in truck crew (2 people) \\
\hline$N_{r}$ & $=$ & One-way traffic count in rural zone ( 470 vehicles $/ \mathrm{hr}$ ) \\
\hline $\mathbf{N}_{\mathbf{s}}$ & $=$ & One-way traffic count in suburban zone ( 780 vehicles/hr) \\
\hline $\mathbf{N}_{\mathbf{u}}$ & $=$ & One-way traffic count in urban zone ( 2800 vehicles $/ \mathrm{hr})$ \\
\hline $\mathbf{P}_{\text {st }}$ & $=$ & Number of people exposed at stops ( 50 people) \\
\hline $\mathrm{PD}_{\mathrm{r}}$ & $=$ & Population density in rural zone $\left(6\right.$ people $\left./ \mathrm{km}^{2}\right)$ \\
\hline $\mathrm{PD}_{\mathrm{s}}$ & $=$ & Population density in suburban zone (719 people $\left./ \mathrm{km}^{2}\right)$ \\
\hline $\mathrm{PD}_{\mathbf{u}}$ & $=$ & Population density in urban zone (3861 people $/ \mathrm{km}^{2}$ ) \\
\hline PDR & $=$ & $\begin{array}{l}\text { Pedestrian ratio (if } R R, R S \text {, or } R U=1.0 \text {, then RADTRAN } 4 \text { sets the value of } \\
\text { PDR to } 1.0 \text {, otherwise the default value of PDR is } 6.0 \text { ) }\end{array}$ \\
\hline PPV & $=$ & Number of people per vehicle ( 2 people/vehicle) \\
\hline $\mathbf{r}$ & $=$ & Distance from shipping container to truck crew $(3.1 \mathrm{~m})$ \\
\hline$r_{\text {st }}$ & $=$ & Distance from shipping container to people exposed at stops (20 m) \\
\hline $\mathbf{R R}$ & $=$ & Building shielding factor in rural zone (1.0) \\
\hline RS & $=$ & Building shielding factor in suburban zone (1.0) \\
\hline RU & $=$ & Building shielding factor in urban zone (1.0) \\
\hline $\mathrm{T}_{\text {st }}$ & $=$ & Stop time $(0.011 \mathrm{hr} / \mathrm{km})$ \\
\hline $\mathrm{V}_{\mathrm{r}}$ & $=$ & Speed in rural zone $(88.49 \mathrm{~km} / \mathrm{hr})$ \\
\hline $\mathrm{V}$ & $=$ & Speed in suburban zone $(40.25 \mathrm{~km} / \mathrm{hr})$ \\
\hline $\mathrm{V}_{\mathbf{u}}$ & $=$ & Speed in urban zone $(24.16 \mathrm{~km} / \mathrm{hr})$ \\
\hline
\end{tabular}


$H_{\min , \max }=\int_{x=\min }^{x=\max }\left(\int_{r=x}^{r=\infty} \frac{d r}{r\left(r^{2}-x^{2}\right)^{1 / 2}}\right] d x=\frac{\pi}{2} \cdot \ln \left[\frac{\max }{\min }\right]$

$\mathrm{H}_{\text {min,max }}$ is unitless

$Z_{x, \infty}=\int_{r=x}^{r=\infty} \frac{d r}{r\left(r^{2}-x^{2}\right)^{1 / 2}}=\frac{\pi}{2} \cdot \frac{1}{x}$

$\mathrm{Z}_{\mathrm{x}, \infty}$ has units of $\mathrm{m}^{-1}$

$Y(\min )=\int_{r=4}^{r=\infty} \frac{d r}{r^{2}}=\frac{1}{4 m}$

$\mathrm{Y}(\mathrm{min})$ has units of $\mathrm{m}^{-1}$

$Y(x)=\int_{r=y}^{r=\infty} \frac{d r}{r^{2}}=\frac{1}{y}$

where $\mathrm{y}=\mathrm{x} \mathrm{km} / \mathrm{hr} \cdot \frac{1000 \mathrm{~m}}{1 \mathrm{~km}} \cdot \frac{1 \mathrm{hr}}{3600 \mathrm{~s}} \cdot 2 \mathrm{~s}$

$Y(x)$ has units of $m^{-1}$ 


\subsubsection{Crew Dose - Truck}

RADTRAN 4 first performs a regulatory check to determine if the dose rate for the crew exceeds $2 \mathrm{mrem} / \mathrm{hr}$, the regulatory limit. If the dose rate for the crew exceeds $2 \mathrm{mrem} / \mathrm{hr}$, then RADTRAN 4 resets the dose rate to $2 \mathrm{mrem} / \mathrm{hr}$ for the crew dose calculations.

Dose $=\frac{\mathrm{k}_{0} \cdot \mathrm{DR} \mathrm{R}_{\mathrm{p}}}{\mathrm{r}^{2}}$

Dose $=\frac{6.25 \mathrm{~m}^{2} \cdot 14 \mathrm{mrem} / \mathrm{hr}}{(3.1 \mathrm{~m})^{2}}=9.11 \mathrm{mrem} / \mathrm{hr}$

\subsubsection{Rural Crew Dose - Truck.}

Dose $=\frac{2 \mathrm{mrem} / \mathrm{hr}}{\mathrm{V}_{\mathrm{r}}} \cdot$ DIST $\cdot \mathrm{N}_{\mathrm{c}}$

Dose $=\frac{2 \mathrm{mrem} / \mathrm{hr}}{88.49 \mathrm{~km} / \mathrm{hr}} \cdot 1 \mathrm{~km} \cdot 2$ persons $\cdot \frac{1 \mathrm{rem}}{1000 \mathrm{mrem}}$

Dose $=4.52 \mathrm{E}-5$ person $-\mathrm{rem}$ 


\subsubsection{Suburban Crew Dose - Truck.}

Dose $=\frac{2 \mathrm{mrem} / \mathrm{hr}}{\mathrm{V}_{\mathrm{s}}} \cdot \operatorname{DIST} \cdot \mathrm{N}_{\mathrm{c}}$

Dose $=\frac{2 \mathrm{mrem} / \mathrm{hr}}{40.25 \mathrm{~km} / \mathrm{hr}} \cdot 1 \mathrm{~km} \cdot 2$ persons $\cdot \frac{1 \mathrm{rem}}{1000 \mathrm{mrem}}$

Dose $=9.94 \mathrm{E}-5$ person $-\mathrm{rem}$

3.4.2.3 Urban Crew Dose - Truck.

Dose $=\frac{2 \mathrm{mrem} / \mathrm{hr}}{\mathrm{V}_{\mathrm{u}}} \cdot \operatorname{DIST} \cdot \mathrm{N}_{\mathrm{c}}$

Dose $=\frac{2 \mathrm{mrem} / \mathrm{hr}}{24.16 \mathrm{~km} / \mathrm{hr}} \cdot 1 \mathrm{~km} \cdot 2$ persons $\cdot \frac{1 \mathrm{rem}}{1000 \mathrm{mrem}}$

Dose $=1.66 \mathrm{E}-4$ person-rem 


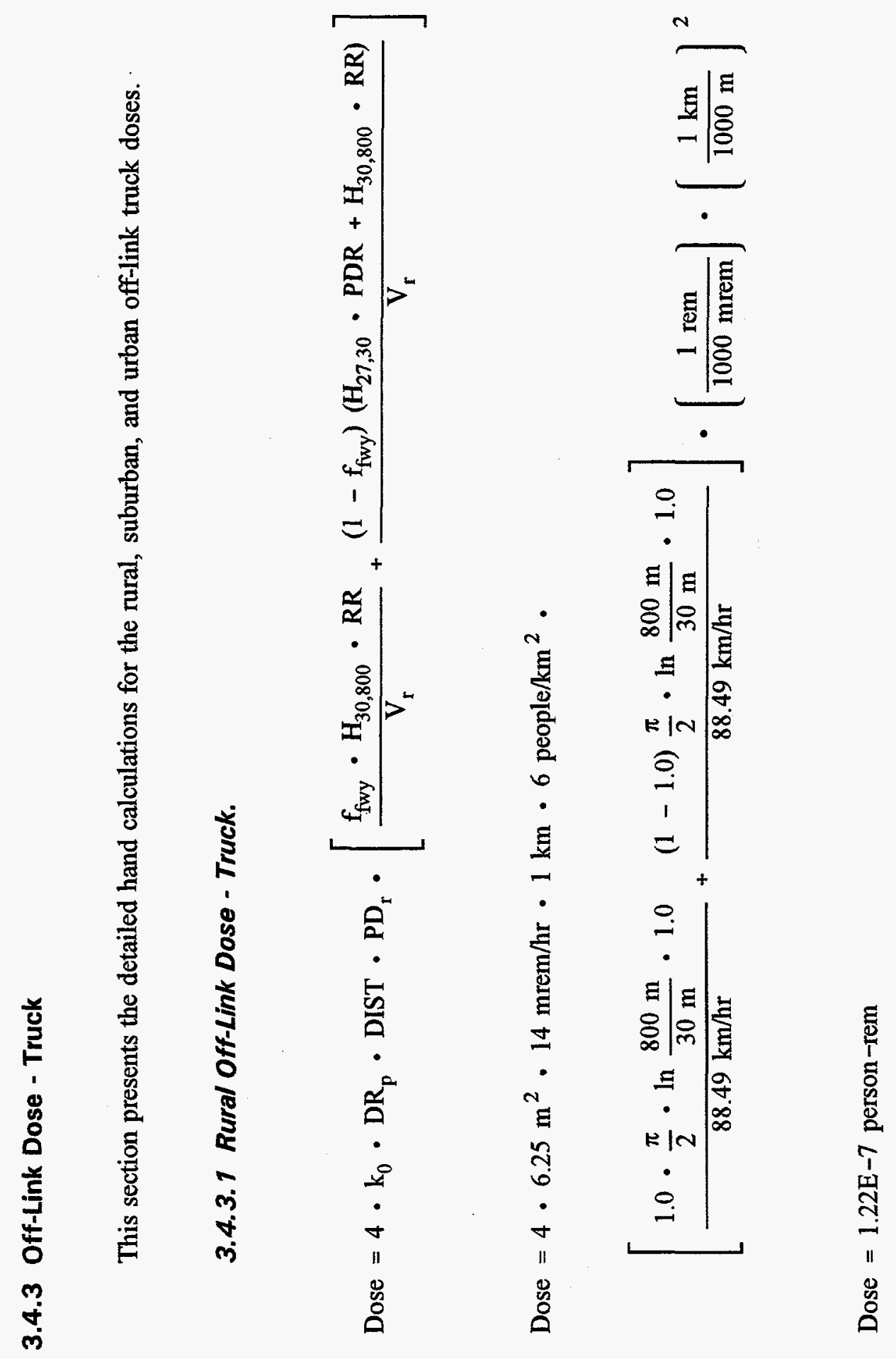




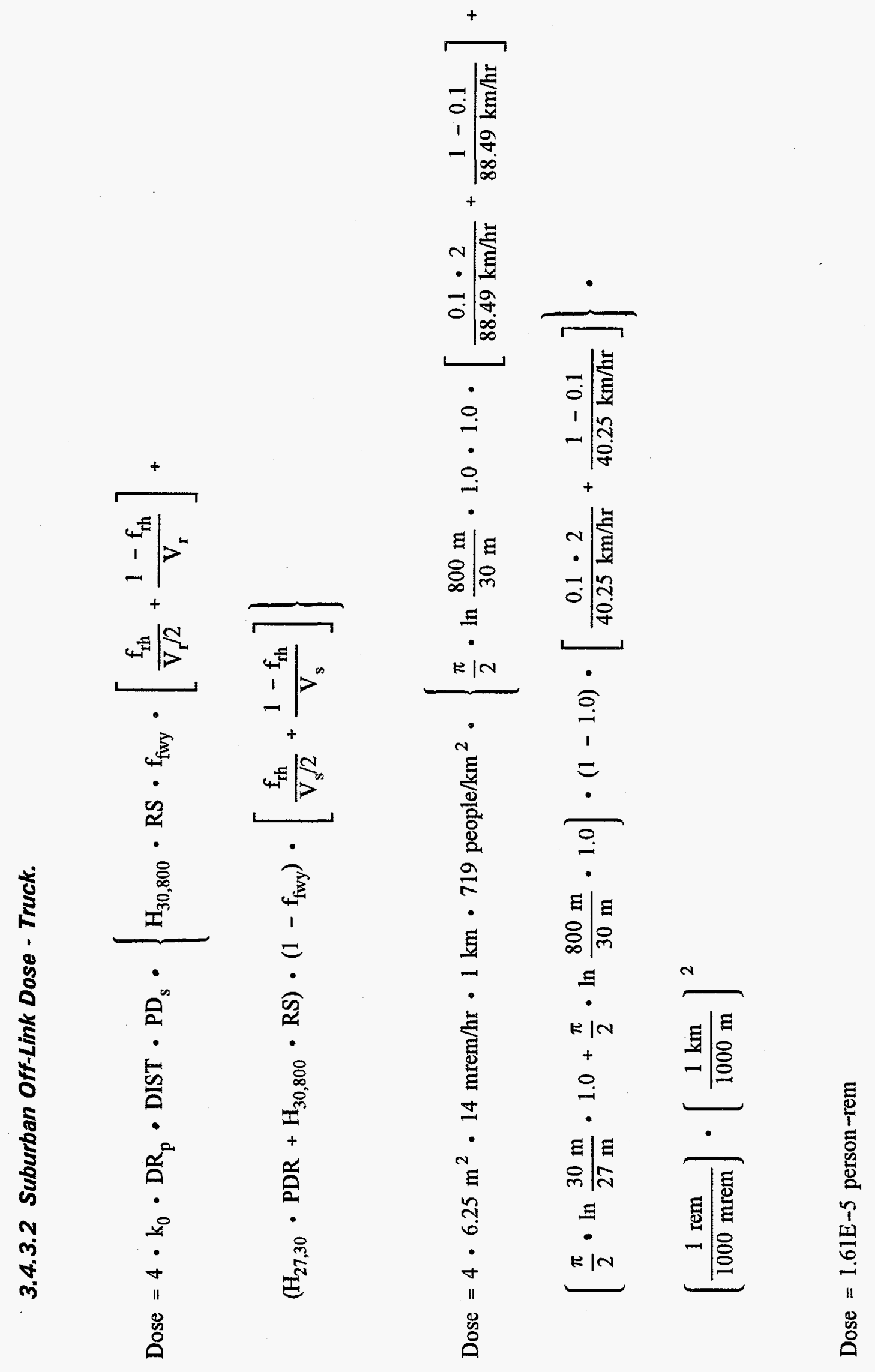



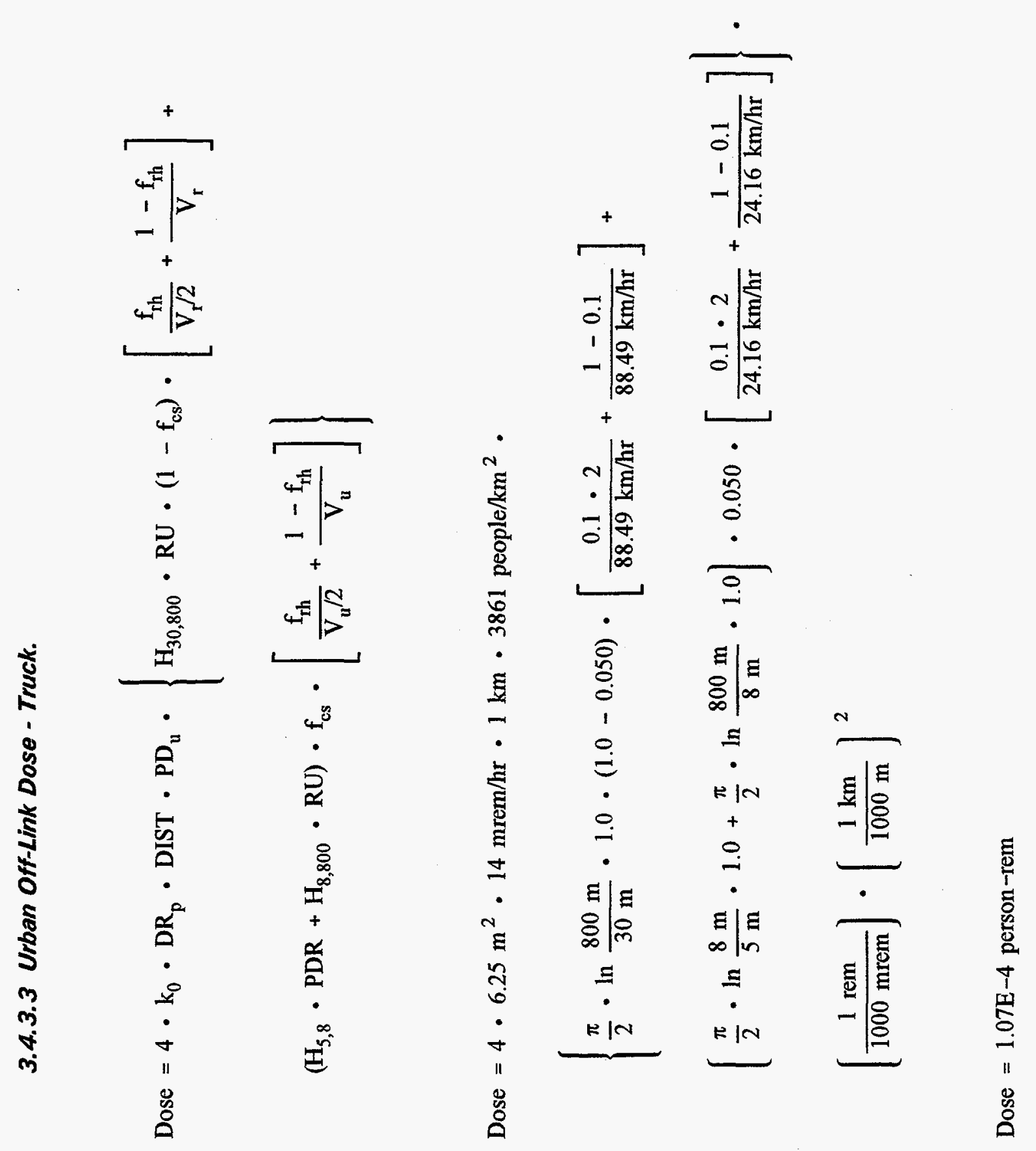


\subsubsection{On-Link Dose - Truck}

The on-link dose is comprised of three parts: (1) the dose to persons traveling in the same direction as the shipment, (2) the dose to persons traveling in the opposite direction as the shipment, and (3) the dose to persons passing the shipment. 

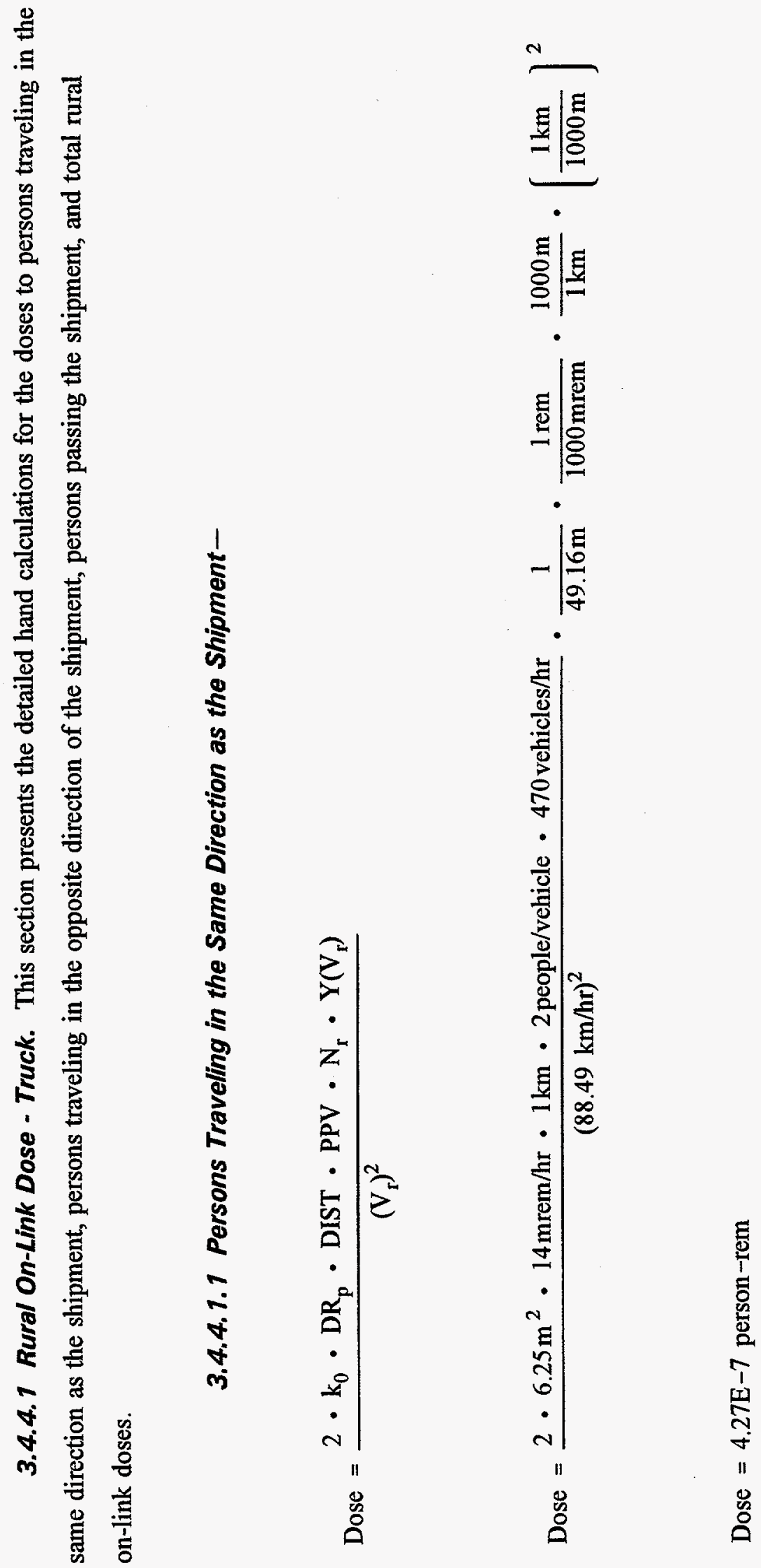


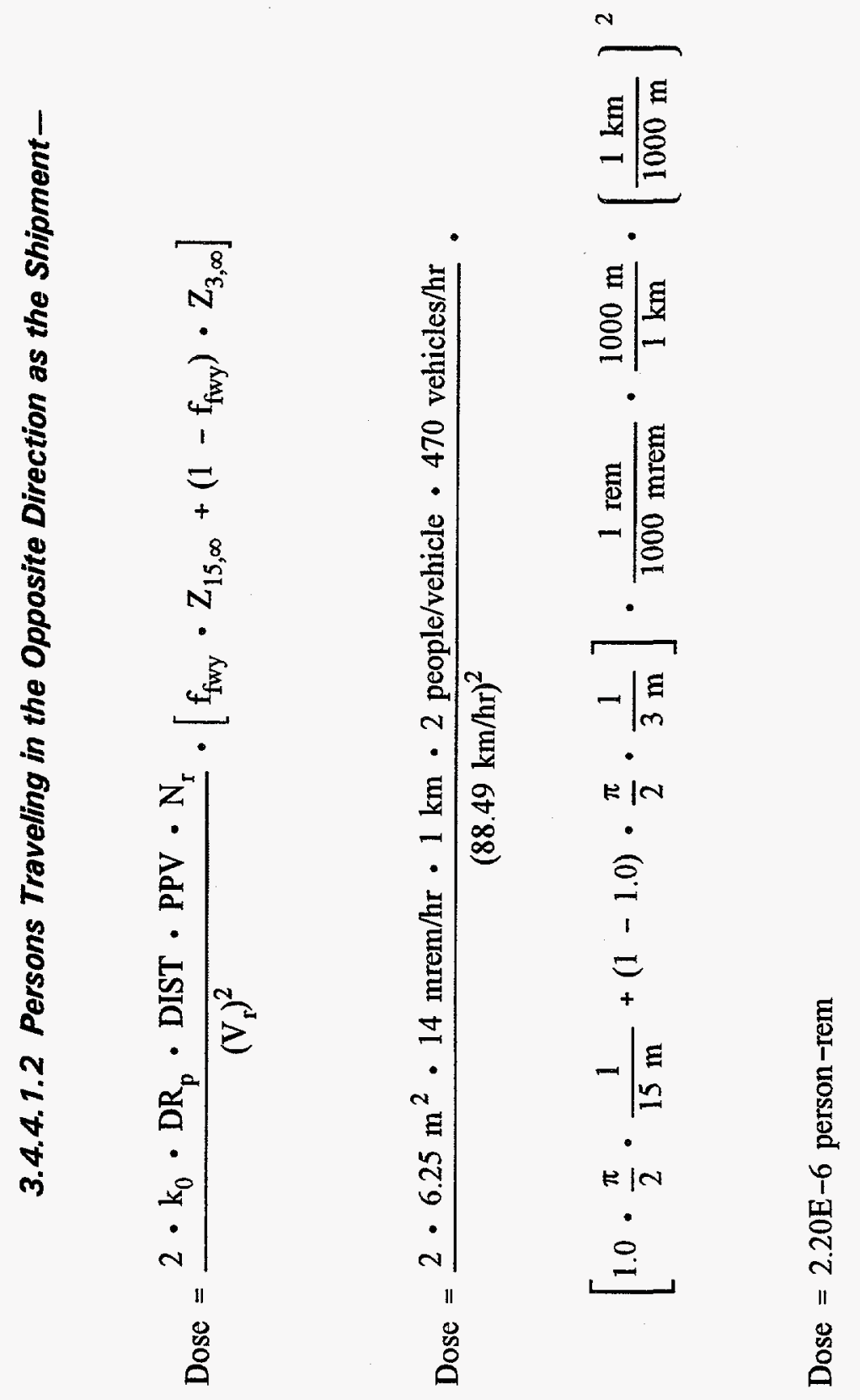




\subsection{Persons Passing the Shipment-}

Dose $=\frac{2 \cdot \mathrm{k}_{0} \cdot \mathrm{DR}_{\mathrm{p}} \cdot \mathrm{DIST} \cdot \mathrm{PPV} \cdot \mathrm{N}_{\mathrm{r}}}{\left(\mathrm{V}_{\mathrm{r}}\right)^{2}} \cdot \frac{1}{2} \cdot\left[\mathrm{Y}(\min )-\mathrm{Y}\left(\mathrm{V}_{\mathrm{r}}\right)\right]$

Dose $=\frac{2 \cdot 6.25 \mathrm{~m}^{2} \cdot 14 \mathrm{mrem} / \mathrm{hr} \cdot 1 \mathrm{~km} \cdot 2 \text { people/vehicle } \cdot 470 \text { vehicles } / \mathrm{hr}}{(88.49 \mathrm{~km} / \mathrm{hr})^{2}} \cdot \frac{1}{2} \cdot\left[\frac{1}{4 \mathrm{~m}}-\frac{1}{49.16 \mathrm{~m}}\right]$.

$$
\frac{1 \mathrm{rem}}{1000 \mathrm{mrem}} \cdot \frac{1000 \mathrm{~m}}{1 \mathrm{~km}} \cdot\left(\frac{1 \mathrm{~km}}{1000 \mathrm{~m}}\right)^{2}
$$

$\stackrel{\omega}{\Delta}$

Dose $=2.41 \mathrm{E}-6$ person $-\mathrm{rem}$

3.4.4.1.4 Total Rural On-Link Dose - Truck-

Total On-Link Dose $=4.27 \mathrm{E}-7$ person-rem $+2.20 \mathrm{E}-6$ person-rem $+2.41 \mathrm{E}-6$ person-rem $=5.04 \mathrm{E}-6$ person-rem 
3.4.4.2 Suburban On-Link Dose - Truck. This section presents the detailed hand calculations for the doses to persons traveling in the same direction as the shipment, persons traveling in the opposite direction of the shipment, persons passing the shipment, and total suburban on-link doses.

\subsection{Persons Traveling in the Same Direction as the Shipment-}

$$
\begin{aligned}
\text { Dose }= & 2 \cdot \mathrm{k}_{0} \cdot \mathrm{DR}_{\mathrm{p}} \cdot \mathrm{DIST} \cdot \mathrm{PPV} \cdot \mathrm{N}_{\mathrm{s}} \cdot\left\{\mathrm{f}_{\mathrm{fwy}} \cdot\left[\frac{\mathrm{f}_{\mathrm{rh}} \cdot 2}{\left(\mathrm{~V}_{\mathrm{r}} / 2\right)^{2}} \cdot \mathrm{Y}\left(\mathrm{V}_{\mathrm{r}} / 2\right)+\frac{1-\mathrm{f}_{\mathrm{rh}}}{\left(\mathrm{V}_{\mathrm{r}}\right)^{2}} \cdot \mathrm{Y}\left(\mathrm{V}_{\mathrm{r}}\right)\right]+\right. \\
& \left.\left(1-\mathrm{f}_{\mathrm{fwy}}\right) \cdot\left[\frac{\mathrm{f}_{\mathrm{rh}} \cdot 2}{\left(\mathrm{~V}_{\mathrm{s}} / 2\right)^{2}} \cdot \mathrm{Y}^{2}\left(\mathrm{~V}_{\mathrm{s}} / 2\right)+\frac{\left(1-\mathrm{f}_{\mathrm{rh}}\right)}{\left(\mathrm{V}_{\mathrm{s}}\right)^{2}} \cdot \mathrm{Y}\left(\mathrm{V}_{\mathrm{s}}\right)\right]\right\}
\end{aligned}
$$

$\stackrel{\omega}{\stackrel{\omega}{\infty}}$

Dose $=2 \cdot 6.25 \mathrm{~m}^{2} \cdot 14 \mathrm{mrem} / \mathrm{hr} \cdot 1 \mathrm{~km} \cdot 2$ people/vehicle $\cdot 780$ vehicles $/ \mathrm{hr} \cdot$

$$
\begin{aligned}
& \left\{1.0 \cdot\left[\frac{0.1 \cdot 2 \cdot 4}{(88.49 \mathrm{~km} / \mathrm{hr})^{2}} \cdot \frac{1}{24.58 \mathrm{~m}}+\frac{(1-0.1)}{(88.49 \mathrm{~km} / \mathrm{hr})^{2}} \cdot \frac{1}{49.16 \mathrm{~m}}\right]+\right. \\
& \left.(1-1.0) \cdot\left[\frac{0.1 \cdot 2 \cdot 4}{(40.25 \mathrm{~km} / \mathrm{hr})^{2}} \cdot \frac{1}{11.18 \mathrm{~m}}+\frac{(1-0.1)}{(40.25 \mathrm{~km} / \mathrm{hr})^{2}} \cdot \frac{1}{22.36 \mathrm{~m})}\right]\right\} \cdot \frac{1 \mathrm{rem}}{1000 \mathrm{mrem}} \cdot \frac{1000 \mathrm{~m}}{1 \mathrm{~km}} \cdot\left[\frac{1 \mathrm{~km}}{1000 \mathrm{~m}}\right]^{2}
\end{aligned}
$$

Dose $=1.77 \mathrm{E}-6$ person - rem 

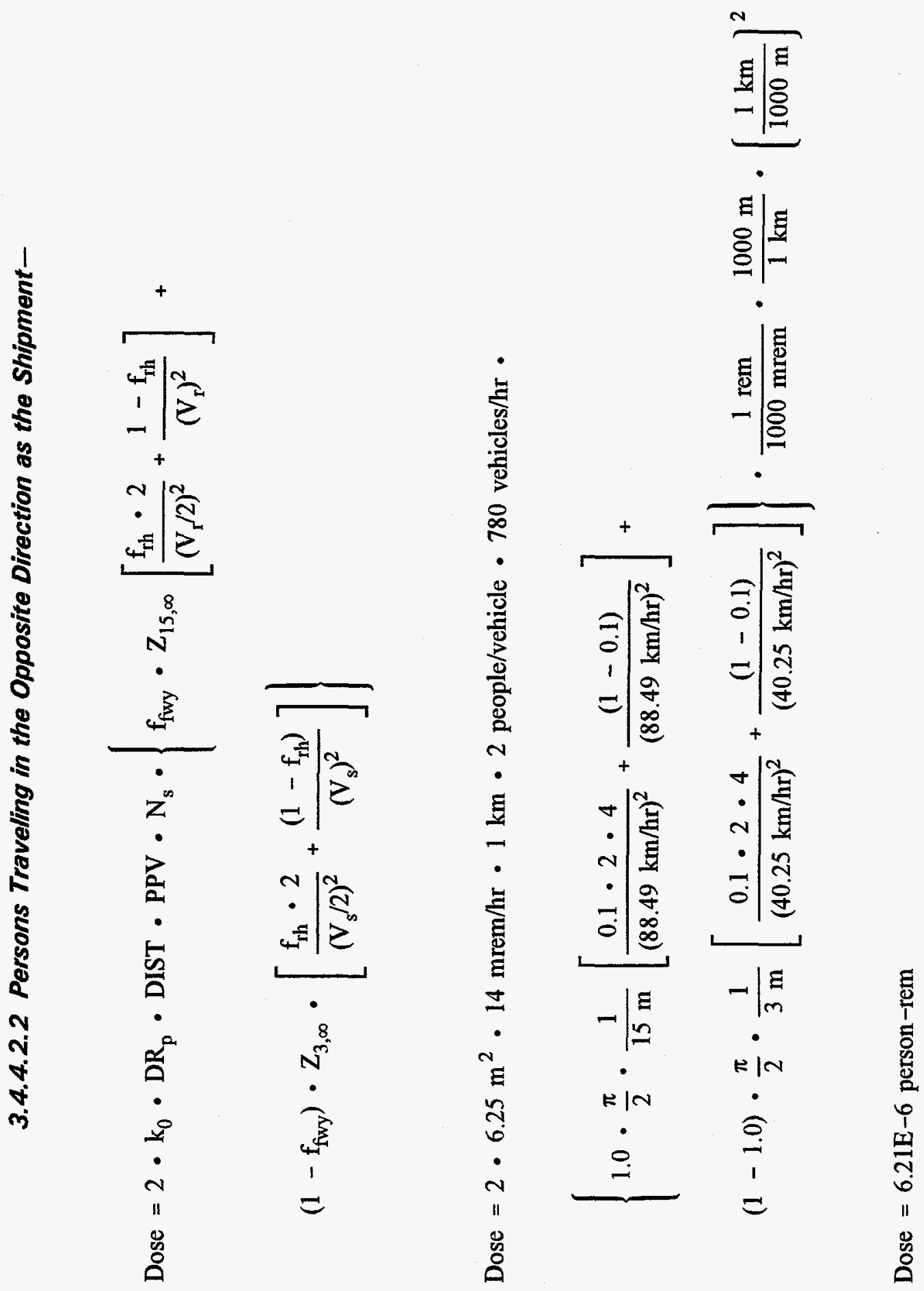

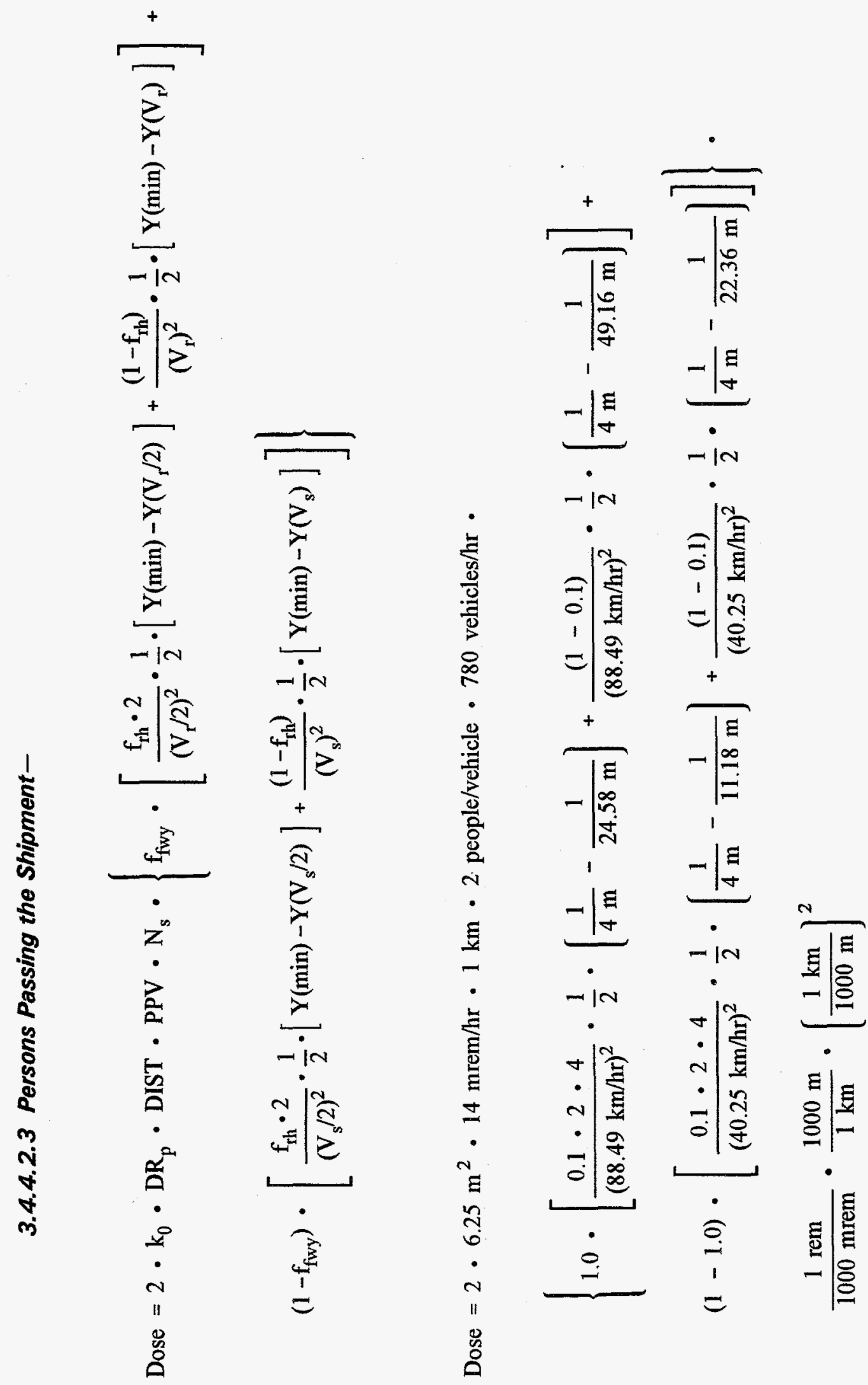

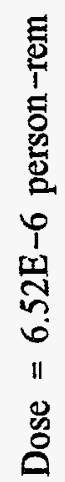




\subsection{Total Suburban On-Link Dose - Truck-}

Total On-Link Dose $=1.77 \mathrm{E}-6$ person-rem $+6.21 \mathrm{E}-6$ person-rem $+6.52 \mathrm{E}-6$ person-rem $=1.45 \mathrm{E}-5$ person $-\mathrm{rem}$ 
3.4.4.3 Urban On-Link Dose - Truck. This section presents the detailed hand calculations for the doses to persons traveling in the same direction as the shipment, persons traveling in the opposite direction of the shipment, persons passing the shipment, and total urban on-link doses.

3.4.4.3.1 Persons Traveling in the Same Direction as the Shipment-

$$
\begin{aligned}
\text { Dose } & =2 \cdot \mathrm{k}_{0} \cdot \mathrm{DR} \mathrm{R}_{\mathrm{p}} \cdot \mathrm{DIST} \cdot \mathrm{PPV} \cdot \mathrm{N}_{\mathrm{u}} \cdot\left\{\left(1-\mathrm{f}_{\mathrm{cs}}\right) \cdot\left[\frac{\mathrm{f}_{\mathrm{rh}} \cdot 2}{\left(\mathrm{~V}_{\mathrm{r} / 2)^{2}}\right.} \cdot \mathrm{Y}\left(\mathrm{V}_{\mathrm{r}} / 2\right)+\frac{\left(1-\mathrm{f}_{\mathrm{rh}}\right)}{\left(\mathrm{V}_{\mathrm{r}}\right)^{2}} \cdot \mathrm{Y}\left(\mathrm{V}_{\mathrm{r}}\right)\right]+\right. \\
& \left.\mathrm{f}_{\mathrm{cs}} \cdot\left[\frac{\mathrm{f}_{\mathrm{rh}} \cdot 2}{\left(\mathrm{~V}_{\mathrm{u}} / 2\right)^{2}} \cdot \mathrm{Y}\left(\mathrm{V}_{\mathrm{u}} / 2\right)+\frac{\left(1-\mathrm{f}_{\mathrm{rh}}\right)}{\left(\mathrm{V}_{\mathrm{u}}\right)^{2}} \cdot \mathrm{Y}\left(\mathrm{V}_{\mathrm{u}}\right)\right]\right\}
\end{aligned}
$$

Dose $=2 \cdot 6.25 \mathrm{~m}^{2} \cdot 14 \mathrm{mrem} / \mathrm{hr} \cdot 1 \mathrm{~km} \cdot 2$ people/vehicle $\cdot 2800$ vehicles $/ \mathrm{hr} \cdot$

$$
\begin{aligned}
& \left\{(1-0.050) \cdot\left[\frac{0.1 \cdot 2 \cdot 4}{(88.49 \mathrm{~km} / \mathrm{hr})^{2}} \cdot \frac{1}{24.58 \mathrm{~m}}+\frac{(1-0.1)}{(88.49 \mathrm{~km} / \mathrm{hr})^{2}} \cdot \frac{1}{49.16 \mathrm{~m}}\right]+\right. \\
& \left.0.050 \cdot\left[\frac{0.1 \cdot 2 \cdot 4}{(24.16 \mathrm{~km} / \mathrm{hr})^{2}} \cdot \frac{1}{6.711 \mathrm{~m}}+\frac{(1-0.1)}{(24.16 \mathrm{~km} / \mathrm{hr})^{2}} \cdot \frac{1}{13.42 \mathrm{~m})}\right]\right\} \cdot \frac{1 \mathrm{rem}}{1000 \mathrm{mrem}} \cdot \frac{1000 \mathrm{~m}}{1 \mathrm{~km}} \cdot\left[\frac{1 \mathrm{~km}}{1000 \mathrm{~m}}\right]^{2}
\end{aligned}
$$

Dose $=2.17 \mathrm{E}-5$ person $-\mathrm{rem}$ 


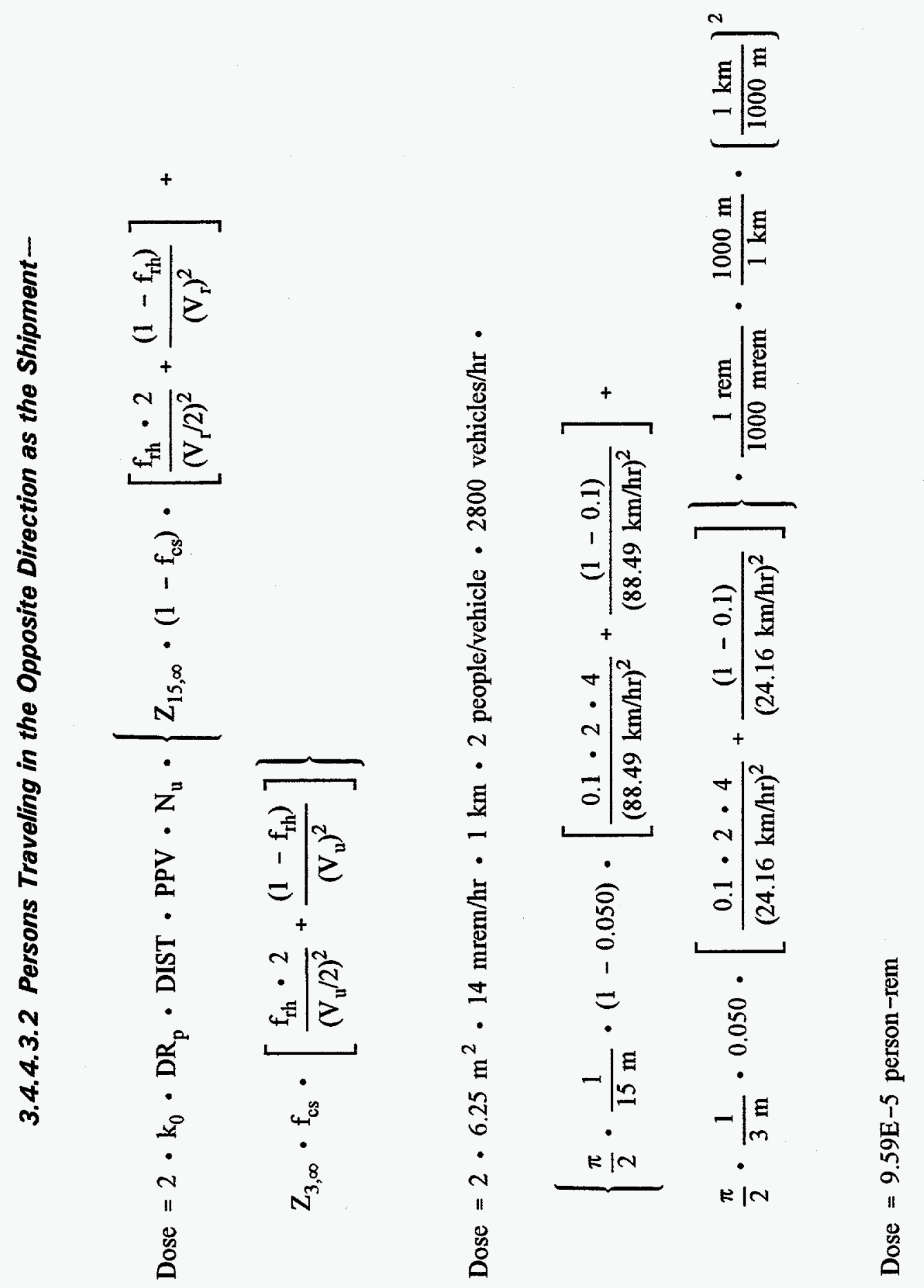




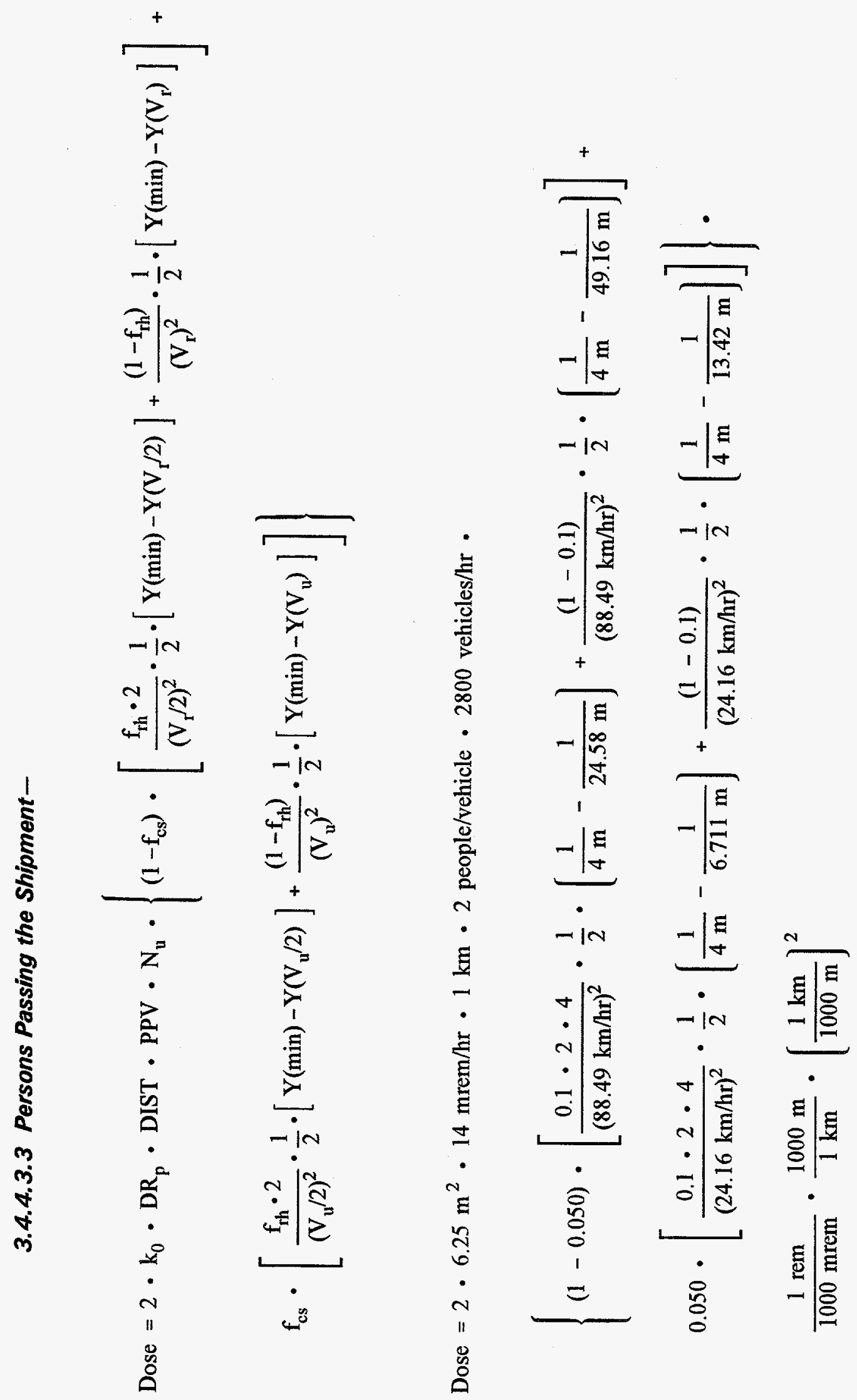

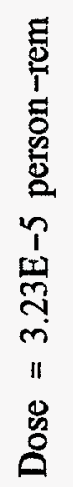




\subsection{Total Urban On-Link Dose - Truck-}

Total On-Link Dose $=2.17 \mathrm{E}-5$ person-rem $+9.59 \mathrm{E}-5$ person-rem $+3.23 \mathrm{E}-5$ person-rem $=1.50 \mathrm{E}-4$ person-rem 


\subsubsection{Dose at Stops - Truck}

This section presents the detailed hand calculations for doses at rural, suburban, and urban stops.

\subsubsection{Rural Stop Dose - Truck.}

Dose $=\frac{\mathrm{k}_{0} \cdot \mathrm{DR}_{\mathrm{p}} \cdot \mathrm{T}_{\mathrm{st}} \cdot \mathrm{P}_{\mathrm{st}} \cdot \mathrm{DIST}}{\left(\mathrm{r}_{\mathrm{st}}\right)^{2}}$

Dose $=\frac{6.25 \mathrm{~m}^{2} \cdot 14 \mathrm{mrem} / \mathrm{hr} \cdot 0.011 \mathrm{hr} / \mathrm{km} \cdot 50 \text { people } \cdot 1 \mathrm{~km}}{(20 \mathrm{~m})^{2}} \cdot \frac{1 \mathrm{rem}}{1000 \mathrm{mrem}}$

Dose $=1.20 \mathrm{E}-4$ person-rem

\subsubsection{Suburban Stop Dose - Truck.}

Dose $=\frac{k_{0} \cdot D R_{p} \cdot T_{s t} \cdot P_{s t} \cdot D I S T}{\left(r_{s t}\right)^{2}}$

Dose $=\frac{6.25 \mathrm{~m}^{2} \cdot 14 \mathrm{mrem} / \mathrm{hr} \cdot 0.011 \mathrm{hr} / \mathrm{km} \cdot 50 \text { people } \cdot 1 \mathrm{~km}}{(20 \mathrm{~m})^{2}} \cdot \frac{1 \mathrm{rem}}{1000 \mathrm{mrem}}$

Dose $=1.20 \mathrm{E}-4$ person-rem 
3.4.5.3 Urban Stop Dose - Truck.

Dose $=\frac{\mathrm{k}_{0} \cdot \mathrm{DR}_{\mathrm{p}} \cdot \mathrm{T}_{\mathrm{st}} \cdot \mathrm{P}_{\mathrm{st}} \cdot \mathrm{DIST}}{\left(\mathrm{r}_{\mathrm{st}}\right)^{2}}$

Dose $=\frac{6.25 \mathrm{~m}^{2} \cdot 14 \mathrm{mrem} / \mathrm{hr} \cdot 0.011 \mathrm{hr} / \mathrm{km} \cdot 50 \text { people } \cdot 1 \mathrm{~km}}{(20 \mathrm{~m})^{2}} \cdot \frac{1 \mathrm{rem}}{1000 \mathrm{mrem}}$

Dose $=1.20 \mathrm{E}-4$ person-rem 


\subsection{Detailed Hand Calculations for Incident-Free Rail Unit Risk Factors}

This section presents the detailed hand calculations for (a) linear and nonlinear rural, suburban, and urban crew doses and (b) off-link and on-link doses.

\subsubsection{List of Symbols - Truck}

\begin{tabular}{|c|c|c|}
\hline$d_{e}$ & $=$ & Effective package dimension $(3 \mathrm{~m})$ \\
\hline $\mathrm{DR}_{\mathrm{p}}$ & $=$ & Package dose rate $(14 \mathrm{mrem} / \mathrm{hr})$ \\
\hline DIST & $=$ & Distance $(1 \mathrm{~km})$ \\
\hline EF & $=$ & Exposure factor $(0.16 \mathrm{hr} / \mathrm{km})$ \\
\hline $\mathrm{k}_{0}$ & $=$ & Package shape factor $\left(6.25 \mathrm{~m}^{2}\right)$ \\
\hline $\mathrm{k}_{0}^{\prime}$ & $=$ & Line-source package shape factor $(2.5 \mathrm{~m})$ \\
\hline MINCL & $=$ & Minimum number of rail classifications or inspections (2) \\
\hline $\mathrm{N}_{\mathbf{r}}$ & $=$ & One-way traffic count in rural zone ( 1 vehicle/hr) \\
\hline $\mathbf{N}_{\mathbf{s}}$ & $=$ & One-way traffic count in suburban zone ( 5 vehicles/hr) \\
\hline $\mathbf{N}_{\mathbf{u}}$ & $=$ & One-way traffic count in urban zone ( 5 vehicle/hr) \\
\hline $\mathbf{P D}_{\text {st }}$ & $=$ & Population density at stops ( 719 people $/ \mathrm{km}^{2}$ ) \\
\hline $\mathbf{P D}_{\mathbf{r}}$ & $=$ & Population density in rural zone (6 people $/ \mathrm{km}^{2}$ ) \\
\hline $\mathrm{PD}_{\mathrm{s}}$ & $=$ & Population density in suburban zone (719 people $/ \mathrm{km}^{2}$ ) \\
\hline $\mathrm{PD}_{\mathrm{u}}$ & $=$ & Population density in urban zone (3861 people $/ \mathrm{km}^{2}$ ) \\
\hline PPV & $=$ & Number of people per vehicle ( 3 people/vehicle) \\
\hline$r_{\mathrm{a}}$ & $=$ & Minimum distance at stops $(10 \mathrm{~m})$ \\
\hline $\mathbf{r}_{\mathbf{b}}$ & $=$ & Maximum distance at stops $(400 \mathrm{~m})$ \\
\hline $\mathrm{SF}_{\text {st }}$ & $=$ & Shielding factor at stops $(0.1)$ \\
\hline $\mathrm{T}_{\mathrm{a}}$ & $=$ & Distance-independent stop time $(60 \mathrm{hr})$ \\
\hline $\mathrm{T}_{\mathrm{st}}$ & $=$ & Distance-dependent stop time $(0.033 \mathrm{hr} / \mathrm{km})$ \\
\hline $\mathbf{V}_{\mathbf{r}}$ & $=$ & Speed in rural zone $(64.37 \mathrm{~km} / \mathrm{hr})$ \\
\hline $\mathrm{V}_{\mathrm{s}}$ & $=$ & Speed in suburban zone $(40.25 \mathrm{~km} / \mathrm{hr})$ \\
\hline $\mathrm{V}_{\mathrm{u}}$ & $=$ & Speed in urban zone $(24.16 \mathrm{~km} / \mathrm{hr})$ \\
\hline
\end{tabular}


$\mathrm{H}_{\min , \max }=\int_{\mathrm{x}=\min }^{\mathrm{x}=\max }\left[\int_{\mathrm{r}=\mathrm{x}}^{\mathrm{r}=\infty} \frac{\mathrm{dr}}{\mathrm{r}\left(\mathrm{r}^{2}-\mathrm{x}^{2}\right)^{1 / 2}}\right] \mathrm{dx}=\frac{\pi}{2} \cdot \ln \left[\frac{\max }{\min }\right]$

$\mathrm{H}_{\text {min,max }}$ is unitless

$Z_{x, \infty}=\int_{r=x}^{r=\infty} \frac{d r}{r\left(r^{2}-x^{2}\right)^{1 / 2}}=\frac{\pi}{2} \cdot \frac{1}{x}$

$Z_{x, \infty}$ has units of $m^{-1}$ 
3.5.2 Linear Rural, Suburban, and Urban Crew Dose - Rail

$$
\begin{aligned}
& \text { Dose }=\mathrm{k}_{0}^{\prime} \cdot \mathrm{DR}_{\mathrm{p}} \cdot \mathrm{EF} \cdot 0.0018 \cdot \mathrm{DIST} \\
& \mathrm{k}_{0}^{\prime}=1+0.5 \cdot \mathrm{d}_{\mathrm{e}} \\
& \mathrm{k}_{0}^{\prime}=1+0.5 \cdot 3 \mathrm{~m}=2.5 \mathrm{~m} \\
& \text { Dose }=2.5 \mathrm{~m} \cdot 14 \mathrm{mrem} / \mathrm{hr} \cdot 0.16 \text { person }-\mathrm{hr} / \mathrm{m} \cdot \frac{1 \mathrm{rem}}{1000 \mathrm{mrem}} \cdot \frac{0.0018}{\mathrm{~km}} \cdot 1 \mathrm{~km} \\
& \text { Dose }=1.01 \mathrm{E}-5 \text { person-rem }
\end{aligned}
$$

\subsubsection{Nonlinear Rural, Suburban, and Urban Crew Dose - Rail}

$$
\begin{aligned}
& \text { Dose }=\mathrm{k}_{0}^{\prime} \cdot \mathrm{DR}_{\mathrm{p}} \cdot \mathrm{EF} \cdot \mathrm{MINCL} \\
& \text { Dose }=2.5 \mathrm{~m} \cdot 14 \mathrm{mrem} / \mathrm{hr} \cdot 0.16 \text { person- } \mathrm{hr} / \mathrm{m} \cdot 2 \cdot \frac{1 \mathrm{rem}}{1000 \mathrm{mrem}} \\
& \text { Dose }=1.12 \mathrm{E}-2 \text { person-rem }
\end{aligned}
$$




\subsubsection{Off-Link Dose - Rail}

This section presents the detailed hand calculations for rural, suburban, and urban off-link doses.

\subsubsection{Rural Off-Link Dose - Rail.}

Dose $=\frac{4 \cdot \mathrm{k}_{0} \cdot \mathrm{DR}_{\mathrm{p}} \cdot \mathrm{DIST} \cdot \mathrm{H}_{30,800} \cdot \mathrm{PD}_{\mathrm{r}}}{\mathrm{V}_{\mathrm{r}}}$

Dose $=\frac{4 \cdot 6.25 \mathrm{~m}^{2} \cdot 14 \mathrm{mrem} / \mathrm{hr} \cdot 1 \mathrm{~km} \cdot \frac{\pi}{2} \cdot \ln \frac{800 \mathrm{~m}}{30 \mathrm{~m}} \cdot 6 \text { people } / \mathrm{km}^{2}}{64.37 \mathrm{~km} / \mathrm{hr}}$.

$$
\frac{1 \mathrm{rem}}{1000 \mathrm{mrem}} \cdot\left(\frac{1 \mathrm{~km}}{1000 \mathrm{~m}}\right)^{2}
$$

Dose $=1.68 \mathrm{E}-7$ person - rem

\subsubsection{Suburban Off-Link Dose -Rail.}

Dose $=\frac{4 \cdot \mathrm{k}_{0} \cdot \mathrm{DR}_{\mathrm{p}} \cdot \mathrm{DIST} \cdot \mathrm{H}_{30,800} \cdot \mathrm{PD}_{\mathrm{s}}}{\mathrm{V}_{\mathrm{s}}}$

Dose $=\frac{4 \cdot 6.25 \mathrm{~m}^{2} \cdot 14 \mathrm{mrem} / \mathrm{hr} \cdot 1 \mathrm{~km} \cdot \frac{\pi}{2} \cdot \ln \frac{800 \mathrm{~m}}{30 \mathrm{~m}} \cdot 719 \text { people } / \mathrm{km}^{2}}{40.25 \mathrm{~km} / \mathrm{hr}}$.

$$
\frac{1 \mathrm{rem}}{1000 \mathrm{mrem}} \cdot\left(\frac{1 \mathrm{~km}}{1000 \mathrm{~m}}\right)^{2}
$$

Dose $=3.22 \mathrm{E}-5$ person $-\mathrm{rem}$ 


\subsubsection{Urban Off-Link Dose - Rail.}

Dose $=\frac{4 \cdot \mathrm{k}_{0} \cdot \mathrm{DR}_{\mathrm{p}} \cdot \mathrm{DIST} \cdot \mathrm{H}_{30,800} \cdot \mathrm{PD}_{\mathrm{u}}}{\mathrm{V}_{\mathrm{u}}}$

Dose $=\frac{4 \cdot 6.25 \mathrm{~m}^{2} \cdot 14 \mathrm{mrem} / \mathrm{hr} \cdot 1 \mathrm{~km} \cdot \frac{\pi}{2} \cdot \ln \frac{800 \mathrm{~m}}{30 \mathrm{~m}} \cdot 3861 \text { people } / \mathrm{km}^{2}}{24.16 \mathrm{~km} / \mathrm{hr}}$.

$\frac{1 \mathrm{rem}}{1000 \mathrm{mrem}} \cdot\left(\frac{1 \mathrm{~km}}{1000 \mathrm{~m}}\right)^{2}$

Dose $=2.88 \mathrm{E}-4$ person $-\mathrm{rem}$ 


\subsubsection{On-Link Dose - Rail}

This section presents the detailed hand calculations for rural, suburban, and urban on-link doses.

\subsubsection{Rural On-Link Dose - Rail.}

Dose $=\frac{2 \cdot \mathrm{k}_{0} \cdot \mathrm{DR}_{\mathrm{p}} \cdot \mathrm{DIST} \cdot \mathrm{PPV} \cdot \mathrm{N}_{\mathrm{r}} \cdot \mathrm{Z}_{3, \infty}}{\left(\mathrm{V}_{\mathrm{r}}\right)^{2}}$

Dose $=\frac{2 \cdot 6.25 \mathrm{~m}^{2} \cdot 14 \mathrm{mrem} / \mathrm{hr} \cdot 1 \mathrm{~km} \cdot 3 \mathrm{people} / \text { vehicle } \cdot 1 \text { vehicle } / \mathrm{hr} \cdot \frac{\pi}{2} \cdot \frac{1}{3 \mathrm{~m}}}{(64.37 \mathrm{~km} / \mathrm{hr})^{2}}$.

$$
\frac{1 \mathrm{rem}}{1000 \mathrm{mrem}} \cdot \frac{1000 \mathrm{~m}}{1 \mathrm{~km}} \cdot\left(\frac{1 \mathrm{~km}}{1000 \mathrm{~m}}\right)^{2}
$$

Dose $=6.63 \mathrm{E}-8$ person - rem

\subsubsection{Suburban On-Link Dose - Rail.}

Dose $=\frac{2 \cdot \mathrm{k}_{0} \cdot \mathrm{DR}_{\mathrm{p}} \cdot \mathrm{DIST} \cdot \mathrm{PPV} \cdot \mathrm{N}_{\mathrm{s}} \cdot \mathrm{Z}_{3, \infty}}{\left(\mathrm{V}_{\mathrm{s}}\right)^{2}}$

Dose $=\frac{2 \cdot 6.25 \mathrm{~m}^{2} \cdot 14 \mathrm{mrem} / \mathrm{hr} \cdot 1 \mathrm{~km} \cdot 3 \text { people } / \text { vehicle } \cdot 5 \text { vehicles } / \mathrm{hr} \cdot \frac{\pi}{2} \cdot \frac{1}{3 \mathrm{~m}}}{(40.25 \mathrm{~km} / \mathrm{hr})^{2}}$.

$$
\frac{1 \mathrm{rem}}{1000 \mathrm{mrem}} \cdot \frac{1000 \mathrm{~m}}{1 \mathrm{~km}} \cdot\left(\frac{1 \mathrm{~km}}{1000 \mathrm{~m}}\right)^{2}
$$

Dose $=8.48 \mathrm{E}-7$ person-rem 


\subsubsection{Urban On-Link Dose - Rail.}

Dose $=\frac{2 \cdot \mathrm{k}_{0} \cdot \mathrm{DR}_{\mathrm{p}} \cdot \mathrm{DIST} \cdot \mathrm{PPV} \cdot \mathrm{N}_{\mathrm{u}} \cdot \mathrm{Z}_{3, \infty}}{\left(\mathrm{V}_{\mathrm{u}}\right)^{2}}$

Dose $=\frac{2 \cdot 6.25 \mathrm{~m}^{2} \cdot 14 \mathrm{mrem} / \mathrm{hr} \cdot 1 \mathrm{~km} \cdot 3 \text { people } / \text { vehicle } \cdot 5 \text { vehicles } / \mathrm{hr} \cdot \frac{\pi}{2} \cdot \frac{1}{3 \mathrm{~m}}}{(24.16 \mathrm{~km} / \mathrm{hr})^{2}}$.

$$
\frac{1 \mathrm{rem}}{1000 \mathrm{mrem}} \cdot \frac{1000 \mathrm{~m}}{1 \mathrm{~km}} \cdot\left(\frac{1 \mathrm{~km}}{1000 \mathrm{~m}}\right)^{2}
$$

Dose $=2.35 \mathrm{E}-6$ person-rem 


\subsubsection{Dose at Stops - Rail}

This section presents the detailed hand calculations for linear and nonlinear rural, suburban, and urban stop doses.

\subsubsection{Linear Rural, Suburban, and Urban Stop Dose - Rail.}

$$
\begin{aligned}
\text { Dose } & =2 \cdot \pi \cdot \mathrm{k}_{0} \cdot \mathrm{DR}_{\mathrm{p}} \cdot \mathrm{T}_{\mathrm{st}} \cdot \mathrm{PD}_{\mathrm{st}} \cdot \mathrm{DIST} \cdot \mathrm{SF}_{\mathrm{st}} \cdot \ln \frac{\mathrm{r}_{\mathrm{b}}}{\mathrm{r}_{\mathrm{a}}} \\
\text { Dose } & =2 \cdot \pi \cdot 6.25 \mathrm{~m}^{2} \cdot 14 \mathrm{mrem} / \mathrm{hr} \cdot 0.033 \mathrm{hr} / \mathrm{km} \cdot 719 \mathrm{people} / \mathrm{km}^{2} \cdot 1 \mathrm{~km} \cdot 0.1 \cdot \\
& \ln \frac{400 \mathrm{~m}}{10 \mathrm{~m}} \cdot \frac{1 \mathrm{rem}}{1000 \mathrm{mrem}} \cdot\left(\frac{1 \mathrm{~km}}{1000 \mathrm{~m}}\right)^{2}
\end{aligned}
$$

Dose $=4.81 \mathrm{E}-6$ person-rem

\subsubsection{Nonlinear Rural, Suburban, and Urban Stop Dose - Rail.}

Dose $=2 \cdot \pi \cdot \mathrm{k}_{0} \cdot \mathrm{DR}_{\mathrm{p}} \cdot \mathrm{T}_{\mathrm{a}} \cdot \mathrm{PD}_{\mathrm{st}} \cdot \mathrm{SF}_{\mathrm{st}} \cdot \ln \frac{\mathrm{r}_{\mathrm{b}}}{\mathrm{r}_{\mathrm{a}}}$

Dose $=2 \cdot \pi \cdot 6.25 \mathrm{~m}^{2} \cdot 14 \mathrm{mrem} / \mathrm{hr} \cdot 60 \mathrm{hr} \cdot 719$ people $/ \mathrm{km}^{2} \cdot 0.1 \cdot \ln \frac{400 \mathrm{~m}}{10 \mathrm{~m}} \cdot$

$$
\frac{1 \mathrm{rem}}{1000 \mathrm{mrem}} \cdot\left(\frac{1 \mathrm{~km}}{1000 \mathrm{~m}}\right)^{2}
$$

Dose $=8.75 \mathrm{E}-3$ person $-\mathrm{rem}$ 


\section{VALIDATION OF THE ACCIDENT RISKS CALCULATED USING THE RADTRAN 4 COMPUTER CODE}

\subsection{Validation Analysis}

The RADTRAN 4 computer code (Neuhauser and Kanipe 1995) was used to estimate accident risks for the transportation analyses in DOE (1995). The accident risks represent the probability $\times$ consequences for a complete spectrum of accidents.

The purpose of this analysis was to validate the accident risks calculated by RADTRAN 4 by comparing them to hand-calculated values using identical data and equations. The definition of validation used in this analysis was "the test and evaluation of the completed software to ensure compliance with software requirements" (ASME 1989). In the context of this analysis, compliance with software requirements means that the differences between the accident risks calculated by RADTRAN 4 and the hand-calculated accident risks should be small (i.e., within the acceptance criterion established for the validation).

The acceptance criterion for this validation was a percent difference of 5 percent. If the RADTRAN 4 calculated accident risk differed from the hand-calculated accident risk by more than 5 percent, then the result was considered unacceptable. If the RADTRAN 4 calculated accident risk differed from the hand-calculated accident risk by less than 5 percent, then the result was considered acceptable.

Two simple cases were constructed for this validation analysis. In the first case, one radionuclide, cesium-137, was used in the validation analysis and risks were estimated through the groundshine, inhalation, resuspension, cloudshine, and ingestion pathways. This case was designed to test the consequence analysis modules of RADTRAN 4 by setting the accident probability, conditional probabilities, release fractions, aerosolized fractions, and respirable fractions equal to 1.0. The assumptions for the first case are listed below:

- The distance traveled was 1 kilometer $(0.6$ mile $)$ in a rural population zone.

- The accident probability was 1.0 per kilometer $(0.6$ mile).

- The package inventory was $1.0 \mathrm{E}-6 \mathrm{Ci}$ of cesium-137. 
- There was one accident severity class with a conditional probability of 1.0.

- The release fraction, aerosolized fraction, and respirable fraction associated with the accident severity class were 1.0 .

- There were two contaminated areas consisting of 459 square meters (550 square yards) and 1530 square meters (1836 square yards) with dilution factors of $3.42 \mathrm{E}-3 \mathrm{~s} / \mathrm{m}^{3}$ and $1.72 \mathrm{E}-3 \mathrm{~s} / \mathrm{m}^{3}$, respectively.

The accident risks calculated by the RADTRAN 4 computer code for the first case are listed in Table 4-1. Appendix B contains the RADTRAN 4 output for the first case.

The equations used to calculate the accident risks are listed in Chapter 5 of the RADTRAN 4 Technical Manual (Neuhauser and Kanipe 1995). The accident risks estimated using hand calculations are listed in Table 4-1. Section 4.4 contains the detailed hand calculations used to estimate these accident risks. Table 4-1 also contains the percent differences between the RADTRAN 4 and the hand-calculated accident risks.

The second case analyzed in the validation analysis was designed to test the probabilistic modules of RADTRAN 4. It was identical to the first case, except that an accident probability [1.0E-7 accidents per kilometer (1.6E-7 per mile)], conditional probabilities, release fractions, aerosolized fractions, and respirable fractions were incorporated into the analysis (see Table 4-2). As in the first case, risks were estimated through the groundshine, inhalation, resuspension, cloudshine, and ingestion pathways.

To calculate to radiation doses through the groundshine pathway, the groundshine dose calculated in the first case (see Table 4-1) was multiplied by the product of the accident rate, the conditional probability, release fraction, and aerosolized fraction for each severity class. The total groundshine dose is the sum of groundshine doses for each severity class.

To calculate to radiation doses through the inhalation pathway, the inhalation dose calculated in the first case (see Table 4-1) was multiplied by the product of the accident rate, the conditional probability, release fraction, aerosolized fraction, and respirable fraction for each severity class. The total inhalation dose is the sum of inhalation doses for each severity class. 
To calculate to radiation doses through the resuspension pathway, the resuspension dose calculated in the first case (see Table 4-1) was multiplied by the product of the accident rate, the conditional probability, release fraction, aerosolized fraction, and respirable fraction for each severity class. The total resuspension dose is the sum of resuspension doses for each severity class.

To calculate to radiation doses through the cloudshine pathway, the cloudshine dose calculated in the first case (see Table 4-1) was multiplied by the product of the accident rate, the conditional probability, release fraction, and aerosolized fraction for each severity class. The total cloudshine dose is the sum of cloudshine doses for each severity class.

To calculate to radiation doses through the ingestion pathway, the ingestion dose calculated in the first case (see Table 4-1) was multiplied by the product of the accident rate, the conditional probability, release fraction, and aerosolized fraction for each severity class. The total ingestion dose is the sum of ingestion doses for each severity class.

Appendix B contains the RADTRAN 4 output for the second case. Table 4-3 summarizes the radiation doses for each severity class and pathway. Table 4-4 contains the percent differences between the RADTRAN 4- and the hand-calculated accident risks.

\subsection{Results of Validation Analysis}

For the first case, designed to test the consequence analysis modules of RADTRAN 4, the minimum percent difference observed in Table 4-1 was zero, which meant that RADTRAN 4 and the hand calculation yielded identical results. The maximum percent difference observed in Table 4-1 was -0.38 percent, well within the 5 percent validation criterion.

For the second case, designed to test the probabilistic modules of RADTRAN 4, the minimum percent difference observed in Table 4-4 was also zero, which meant that RADTRAN 4 and the hand calculation yielded identical results. The maximum percent difference observed in Table 4-4 was -0.17 percent, well within the 5 percent validation criterion.

These results show that the differences between the accident risks estimated by RADTRAN 4 and the accident risks estimated using hand calculations are small and are within the acceptance 
criterion established for the validation. Therefore, the accident risks generated using the RADTRAN 4 computer code are considered validated.

Table 4-1. RADTRAN 4-calculated and hand-calculated accident risks for case one.

\begin{tabular}{llll}
\hline \multirow{2}{*}{ Pathway } & \multicolumn{2}{c}{ Accident risks (person-rem) } & \\
\cline { 2 - 3 } & RADTRAN 4 & Hand-calculated & Percent difference \\
\hline Groundshine & $1.35 \mathrm{E}-7$ & $1.35 \mathrm{E}-7$ & 0.0 \\
Inhalation & $2.84 \mathrm{E}-10$ & $2.84 \mathrm{E}-10$ & 0.0 \\
Resuspension & $1.25 \mathrm{E}-9$ & $1.25 \mathrm{E}-9$ & 0.0 \\
Cloudshine & $2.61 \mathrm{E}-12$ & $2.62 \mathrm{E}-12$ & -0.38 \\
Ingestion & $5.23 \mathrm{E}-7$ & $5.24 \mathrm{E}-7$ & -0.19 \\
\hline
\end{tabular}

Table 4-2. Conditional probabilities, release fractions, aerosolized fractions, and respirable fractions for case two.

\begin{tabular}{|c|c|c|c|c|}
\hline Severity class & Conditional probability & Release fraction & Aerosolized fraction & Respirable fraction \\
\hline 1 & $9.94 \mathrm{E}-01$ & 0.00 & 0.00 & $5.00 \mathrm{E}-01$ \\
\hline 2 & $2.72 \mathrm{E}-03$ & $1.00 \mathrm{E}-01$ & $4.00 \mathrm{E}-04$ & $5.00 \mathrm{E}-01$ \\
\hline 3 & $5.54 \mathrm{E}-04$ & $1.00 \mathrm{E}+00$ & $4.00 \mathrm{E}-04$ & $5.00 \mathrm{E}-01$ \\
\hline 4 & $1.79 \mathrm{E}-09$ & $1.00 \mathrm{E}+00$ & $4.00 \mathrm{E}-03$ & $5.00 \mathrm{E}-01$ \\
\hline 5 & $1.23 \mathrm{E}-03$ & $3.00 \mathrm{E}-02$ & $4.00 \mathrm{E}-04$ & $5.00 \mathrm{E}-01$ \\
\hline 6 & $5.01 \mathrm{E}-07$ & $1.00 \mathrm{E}-01$ & $4.00 \mathrm{E}-04$ & $5.00 \mathrm{E}-01$ \\
\hline 7 & $1.02 \mathrm{E}-07$ & $1.00 \mathrm{E}+00$ & $4.00 \mathrm{E}-04$ & $5.00 \mathrm{E}-01$ \\
\hline 8 & $3.29 \mathrm{E}-13$ & $1.00 \mathrm{E}+00$ & $4.00 \mathrm{E}-03$ & $5.00 \mathrm{E}-01$ \\
\hline 9 & $7.95 \mathrm{E}-04$ & $3.00 \mathrm{E}-02$ & $4.00 \mathrm{E}-04$ & $5.00 \mathrm{E}-01$ \\
\hline 10 & $3.26 \mathrm{E}-07$ & $1.00 \mathrm{E}-01$ & $4.00 \mathrm{E}-04$ & $5.00 \mathrm{E}-01$ \\
\hline 11 & $6.63 \mathrm{E}-08$ & $1.00 \mathrm{E}+00$ & $4.00 \mathrm{E}-04$ & $5.00 \mathrm{E}-01$ \\
\hline 12 & $2.14 \mathrm{E}-13$ & $1.00 \mathrm{E}+00$ & $4.00 \mathrm{E}-03$ & $5.00 \mathrm{E}-01$ \\
\hline$\overline{13}$ & $6.14 \mathrm{E}-04$ & $1.00 \mathrm{E}+00$ & $4.00 \mathrm{E}-04$ & $5.00 \mathrm{E}-01$ \\
\hline 14 & $2.53 \mathrm{E}-07$ & $1.00 \mathrm{E}+00$ & $4.00 \mathrm{E}-04$ & $5.00 \mathrm{E}-01$ \\
\hline 15 & $5.16 \mathrm{E}-08$ & $1.00 \mathrm{E}+00$ & $4.00 \mathrm{E}-04$ & $5.00 \mathrm{E}-01$ \\
\hline 16 & $1.64 \mathrm{E}-13$ & $1.00 \mathrm{E}+00$ & $4.00 \mathrm{E}-03$ & $5.00 \mathrm{E}-01$ \\
\hline 17 & $1.25 \mathrm{E}-04$ & $1.00 \mathrm{E}+00$ & $4.00 \mathrm{E}-03$ & $5.00 \mathrm{E}-01$ \\
\hline 18 & $1.08 \mathrm{E}-08$ & $1.00 \mathrm{E}+00$ & $4.00 \mathrm{E}-03$ & $5.00 \mathrm{E}-01$ \\
\hline 19 & $5.30 \mathrm{E}-08$ & $1.00 \mathrm{E}+00$ & $4.00 \mathrm{E}-03$ & $5.00 \mathrm{E}-01$ \\
\hline 20 & $3.46 \mathrm{E}-14$ & $1.00 \mathrm{E}+00$ & $4.00 \mathrm{E}-03$ & $5.00 \mathrm{E}-01$ \\
\hline
\end{tabular}


Table 4-3. Radiation doses by pathway and severity class for case two.

\begin{tabular}{|c|c|c|c|c|c|}
\hline Severity class & $\begin{array}{r}\text { Inhalation dose } \\
\text { (person-rem) }\end{array}$ & $\begin{array}{r}\text { Resuspension dose } \\
\text { (person-rem) }\end{array}$ & $\begin{array}{r}\text { Cloudshine dose } \\
\text { (person-rem) }\end{array}$ & $\begin{array}{r}\text { Ingestion dose } \\
\text { (person-rem) }\end{array}$ & $\begin{array}{r}\text { Groundshine dose } \\
\text { (person-rem) }\end{array}$ \\
\hline 1 & 0.00 & 0.00 & 0.00 & 0.00 & 0.00 \\
\hline 2 & $1.55 \mathrm{E}-24$ & $6.82 \mathrm{E}-24$ & $2.85 \mathrm{E}-26$ & $5.70 \mathrm{E}-21$ & $1.47 \mathrm{E}-21$ \\
\hline 3 & $3.15 \mathrm{E}-24$ & $1.39 \mathrm{E}-23$ & $5.80 \mathrm{E}-26$ & $1.16 \mathrm{E}-20$ & $3.00 \mathrm{E}-21$ \\
\hline 4 & $1.02 \mathrm{E}-28$ & $4.49 \mathrm{E}-28$ & $1.87 \mathrm{E}-30$ & $3.75 \mathrm{E}-25$ & $9.70 \mathrm{E}-26$ \\
\hline 5 & $2.10 \mathrm{E}-25$ & $9.26 \mathrm{E}-25$ & $3.86 \mathrm{E}-27$ & $7.73 \mathrm{E}-22$ & $2.00 \mathrm{E}-22$ \\
\hline 6 & $2.85 \mathrm{E}-28$ & $1.26 \mathrm{E}-27$ & $5.24 \mathrm{E}-30$ & $1.05 \mathrm{E}-24$ & $2.72 \mathrm{E}-25$ \\
\hline 7 & $5.80 \mathrm{E}-28$ & $2.56 \mathrm{E}-27$ & $1.07 \mathrm{E}-29$ & $2.14 \mathrm{E}-24$ & $5.53 \mathrm{E}-25$ \\
\hline 8 & $1.87 \mathrm{E}-32$ & $8.25 \mathrm{E}-32$ & $3.44 \mathrm{E}-34$ & $6.89 \mathrm{E}-29$ & $1.78 \mathrm{E}-29$ \\
\hline 9 & $1.36 \mathrm{E}-25$ & $5.98 \mathrm{E}-25$ & $2.50 \mathrm{E}-27$ & $5.00 \mathrm{E}-22$ & $1.29 \mathrm{E}-22$ \\
\hline 10 & $1.85 \mathrm{E}-28$ & $8.18 \mathrm{E}-28$ & $3.41 \mathrm{E}-30$ & $6.83 \mathrm{E}-25$ & $1.77 \mathrm{E}-25$ \\
\hline 11 & $3.77 \mathrm{E}-28$ & $1.66 \mathrm{E}-27$ & $6.94 \mathrm{E}-30$ & $1.39 \mathrm{E}-24$ & $3.59 \mathrm{E}-25$ \\
\hline 12 & $1.22 \mathrm{E}-32$ & $5.37 \mathrm{E}-32$ & $2.24 \mathrm{E}-34$ & $4.48 \mathrm{E}-29$ & $1.16 \mathrm{E}-29$ \\
\hline 13 & $3.49 \mathrm{E}-24$ & $1.54 \mathrm{E}-23$ & $6.42 \mathrm{E}-26$ & $1.29 \mathrm{E}-20$ & $3.33 \mathrm{E}-21$ \\
\hline 14 & $1.44 \mathrm{E}-27$ & $6.35 \mathrm{E}-27$ & $2.65 \mathrm{E}-29$ & $5.30 \mathrm{E}-24$ & 1.37E-24 \\
\hline 15 & $2.94 \mathrm{E}-28$ & $1.29 \mathrm{E}-27$ & $5.40 \mathrm{E}-30$ & $1.08 \mathrm{E}-24$ & $2.80 \mathrm{E}-25$ \\
\hline 16 & $9.33 E-33$ & 4.11E-32 & $1.72 \mathrm{E}-34$ & $3.44 \mathrm{E}-29$ & $8.89 \mathrm{E}-30$ \\
\hline 17 & $7.11 \mathrm{E}-24$ & $3.14 \mathrm{E}-23$ & $1.31 \mathrm{E}-25$ & $2.62 \mathrm{E}-20$ & $6.77 \mathrm{E}-21$ \\
\hline 18 & $6.14 \mathrm{E}-28$ & $2.71 \mathrm{E}-27$ & $1.13 \mathrm{E}-29$ & $2.26 \mathrm{E}-24$ & $5.85 \mathrm{E}-25$ \\
\hline 19 & $3.02 \mathrm{E}-27$ & $1.33 \mathrm{E}-26$ & $5.54 \mathrm{E}-29$ & $1.11 \mathrm{E}-23$ & $2.87 \mathrm{E}-24$ \\
\hline 20 & $1.97 \mathrm{E}-33$ & $8.68 \mathrm{E}-33$ & $3.62 E-35$ & $7.25 \mathrm{E}-30$ & $1.88 \mathrm{E}-30$ \\
\hline Total & $1.57 \mathrm{E}-23$ & $6.90 \mathrm{E}-23$ & $2.88 \mathrm{E}-25$ & 5.77E-20 & $1.49 \mathrm{E}-20$ \\
\hline
\end{tabular}

Table 4-4. RADTRAN 4-calculated and hand-calculated accident risks for case two.

\begin{tabular}{llll}
\hline & \multicolumn{2}{c}{ Accident risks (person-rem) } & \\
\cline { 2 - 3 } \multicolumn{1}{c}{ Pathway } & RADTRAN 4 & Hand-calculated & Percent difference \\
\hline Groundshine & $1.49 \mathrm{E}-20$ & $1.49 \mathrm{E}-20$ & 0.0 \\
Inhalation & $1.57 \mathrm{E}-23$ & $1.57 \mathrm{E}-23$ & 0.0 \\
Resuspension & $6.91 \mathrm{E}-23$ & $6.90 \mathrm{E}-23$ & 0.14 \\
Cloudshine & $2.88 \mathrm{E}-25$ & $2.88 \mathrm{E}-25$ & 0.0 \\
Ingestion & $5.76 \mathrm{E}-20$ & $5.77 \mathrm{E}-20$ & -0.17 \\
\hline
\end{tabular}




\subsection{Critiques of RADTRAN}

As part of this validation analysis, the following documents were examined for specific comments on the accident risk conceptual models used in RADTRAN:

- Public comments contained in Volume II of the Final Environmental Impact Statement on the Transportation of Radioactive Materials By Air and Other Modes (NRC 1977a)

- The Latest Nuclear Dilemma: Waste Shipment Peril Explored (Resnikoff et al. 1982)

- Analysis of Recent Council on Economic Priorities Newsletter (Jefferson et al. 1982)

- $\quad$ Transporting Spent Nuclear Fuel Allegations and Responses (Jefferson 1983)

- The Next Nuclear Gamble: Transportation and Storage of Nuclear Waste (Resnikoff 1983)

- $\quad$ Probabilistic Risk Assessment and Nuclear Waste Transportation: A Case Study of the Use of RADTRAN in the 1986 Environmental Assessment for Yucca Mountain (Resnikoff 1990)

- A Comparison of RISKIND and RADTRAN 4 (Brumburgh and Alesso 1993)

It should be noted that there have been many comments on the accident rates, conditional probabilities, and release fractions used in specific analyses performed using RADTRAN (i.e., see Resnikoff 1990). These comments questioned the data that were used in the analyses, not the conceptual models contained in RADTRAN. Therefore, they are not addressed in this validation analysis.

Based on these documents, four specific comments on the accident risk conceptual models used in RADTRAN were identified (some of these comments do not apply to the current version of RADTRAN, but do apply to previous versions of RADTRAN): 
1. The health effects models in RADTRAN are out of date.

2. The consequence models in RADTRAN are not detailed enough and should be more realistic.

3. The inhalation dose conversion factors in RADTRAN are inappropriate.

4. The clean-up criteria used in RADTRAN are inappropriate.

These comments were evaluated for their applicability to RADTRAN 4. Responses to the comments are listed below:

1. It is correct that the health effects models in RADTRAN are based on the Reactor Safety Study (NRC 1975). However, RADTRAN provides sufficient data so that health effects may be estimated using more current methods outside the code. In DOE (1995), health effects were estimated from the radiation doses calculated using RADTRAN 4 by using the risk coefficients recommended by the International Commission on Radiological Protection in ICRP Publication 60 (ICRP 1991).

2. The consequence models in RADTRAN are meant to be assessment level models. In DOE (1995), more detailed evaluations of the consequences of transportation accidents were conducted using the RISKIND computer code (Yuan et al. 1993).

3. The dose conversion factors used in RADTRAN are based on methods established by the International Commission on Radiological Protection in ICRP-30. In addition, the RADTRAN data base may be modified to use dose conversion factors from other data bases.

4. The value used for the cleanup criteria is an input parameter that may be modified by the user. In DOE (1995), no credit was taken for cleanup. 


\subsection{Detailed Hand Calculations for Accident Risks}

This section provides the hand calculations used to estimate accident risks in order to compare the result of RADTRAN 4 analyses. The section covers risks estimated through the following pathways: groundshine, inhalation, resuspension, cloudshine, and ingestion. Also included is a list of symbols.

\subsubsection{List of Symbols}

AREA $\quad=\quad$ Area associated with the atmospheric dilution factor, DF $\left(459 \mathrm{~m}^{2}\right.$ and $\left.1,530 \mathrm{~m}^{2}\right)$

AREA $_{1} \quad=\quad$ First area $\left(459 \mathrm{~m}^{2}\right)$

$\operatorname{AREA}_{2} \quad=\quad$ Second area $\left(1530 \mathrm{~m}^{2}\right)$

BR $\quad=\quad$ Breathing rate $\left(3.3 \mathrm{E}-4 \mathrm{~m}^{3} / \mathrm{s}\right)$

$\mathrm{DCF}_{\mathrm{imm}}=$ Immersion dose conversion factor $\left(9.71 \mathrm{E}-2 \mathrm{rem}-\mathrm{m}^{3} / \mathrm{Ci}-\mathrm{s}\right)$

$\mathrm{DCF}_{\text {ing }} \quad=\quad$ Ingestion dose conversion factor $(5.0 \mathrm{E}+4 \mathrm{rem} / \mathrm{Ci})$

$\mathrm{DCF}_{\text {inh }} \quad=\quad$ Inhalation dose conversion factor $(3.2 \mathrm{E}+4 \mathrm{rem} / \mathrm{Ci})$

DF $\quad=\quad$ Atmospheric dilution factor associated with area, AREA (3.42E-3 s/m and $1.72 \mathrm{E}-3 \mathrm{~s} / \mathrm{m}^{3}$ )

$\mathrm{DF}_{1} \quad=\quad$ First atmospheric dilution factor $\left(3.42 \mathrm{E}-3 \mathrm{~s} / \mathrm{m}^{3}\right)$

$\mathrm{DF}_{2} \quad=\quad$ Second atmospheric dilution factor $\left(1.72 \mathrm{E}-3 \mathrm{~s} / \mathrm{m}^{3}\right)$

DIST = distance $(1 \mathrm{~km})$

$\mathrm{E}_{\gamma} \quad=\quad$ Gamma energy $(0.596 \mathrm{MeV})$

$\mathrm{F}_{\mathrm{a}} \quad=\quad$ Aerosolized fraction (1.0)

$\mathrm{F}_{\mathrm{r}} \quad=\quad$ Respirable fraction (1.0)

$\Gamma \quad=\quad$ Groundshine dose conversion factor $\left(3.04 \mathrm{E}-4 \mathrm{mrem}-\mathrm{m}^{2} / \mathrm{day}-\mu \mathrm{Ci}-\mathrm{MeV}\right)$

INV $\quad=\quad$ Container inventory $\left(10^{-6} \mathrm{Ci}\right)$

$\mathrm{k}_{1} \quad=\quad$ Rate constant for short term loss from soil $\left(3.16 \mathrm{E}-3 \mathrm{~d}^{-1}\right)$

$\mathrm{k}_{2} \quad=\quad$ Rate constant for long term loss from soil $\left(8.40 \mathrm{E}-5 \mathrm{~d}^{-1}\right)$

$\mathrm{PD} \quad=\quad$ Population density $\left(6\right.$ people $\left./ \mathrm{km}^{2}\right)$

$\mathrm{P}_{\mathrm{sc}} \quad=\quad$ Conditional probability of severity class (1.0)

RATE $=$ Accident rate $(1.0$ accidents $/ \mathrm{km})$

RF $\quad=\quad$ Release fraction associated with severity class (1.0)

$\mathrm{T} \quad=\quad$ Exposure time for groundshine pathway (1.83E +4 days)

TC $\quad$ = Food transfer factor $(3.07 \mathrm{E}-4)$

$\mathrm{v}_{\mathrm{d}} \quad=\quad$ Deposition velocity $(0.01 \mathrm{~m} / \mathrm{s})$ 


\subsubsection{Groundshine Pathway}

For each area, the accident risk through the groundshine pathway is given by:

$$
\begin{gathered}
\text { Dose }=\mathrm{INV} \cdot \mathrm{RATE} \cdot \mathrm{DIST} \cdot \mathrm{P}_{\mathrm{sc}} \cdot \mathrm{RF} \cdot \mathrm{F}_{\mathrm{a}} \cdot \mathrm{DF} \cdot \mathrm{v}_{\mathrm{d}} \cdot \Gamma \cdot \mathrm{E}_{\gamma} \cdot \mathrm{PD} \cdot \mathrm{AREA} \cdot \\
{\left[0.63 \frac{1-\exp \left(-\mathrm{Tk}_{1}\right)}{\mathrm{k}_{1}}+0.37 \frac{1-\exp \left(-\mathrm{Tk}_{2}\right)}{\mathrm{k}_{2}}\right] \cdot\left[\frac{1 \mathrm{~km}}{1000 \mathrm{~m}}\right]^{2}}
\end{gathered}
$$

For the first area $\left(459 \mathrm{~m}^{2}\right)$ with a dilution factor of $3.42 \mathrm{E}-3 \mathrm{~s} / \mathrm{m}^{3}$ :

Dose $=1 \mu \mathrm{Ci} \cdot 1$ accident $/ \mathrm{km} \cdot 1 \mathrm{~km} \cdot 1.0 \cdot 1.0 \cdot 1.0 \cdot 3.42 \mathrm{E}-3 \mathrm{~s} / \mathrm{m}^{3} \cdot 0.01 \mathrm{~m} / \mathrm{s} \cdot 3.04 \mathrm{E}-4 \frac{\mathrm{rem}-\mathrm{m}^{2}}{\mathrm{day}-\mu \mathrm{Ci}-\mathrm{MeV}} \cdot 0.596 \mathrm{MeV} \cdot$

$$
6 \text { people } / \mathrm{km}^{2} \cdot 4.59 \mathrm{E}+2 \mathrm{~m}^{2} \cdot\left[0.63 \frac{1-\exp \left(-1.83 \mathrm{E}+4 \mathrm{~d} \cdot 3.16 \mathrm{E}-3 \mathrm{~d}^{-1}\right)}{3.16 \mathrm{E}-3 \mathrm{~d}^{-1}}+0.37 \frac{1-\exp \left(-1.83 \mathrm{E}+4 \mathrm{~d} \cdot 8.40 \mathrm{E}-5 \mathrm{~d}^{-1}\right)}{8.40 \mathrm{E}-5 \mathrm{~d}^{-1}}\right] \cdot\left[\frac{1 \mathrm{~km}}{1000 \mathrm{~m}}\right]^{2}
$$

Dose $=6.23 \mathrm{E}-8$ person $-\mathrm{rem}$ 
For the second area $\left(1071 \mathrm{~m}^{2}=1530 \mathrm{~m}^{2}-459 \mathrm{~m}^{2}\right)$ with a dilution factor of $1.72 \mathrm{E}-5 \mathrm{~s} / \mathrm{m}^{3}$ :

Dose $=1 \mu \mathrm{Ci} \cdot 1 \mathrm{accident} / \mathrm{km} \cdot 1 \mathrm{~km} \cdot 1.0 \cdot 1.0 \cdot 1.0 \cdot 1.72 \mathrm{E}-3 \mathrm{~s} / \mathrm{m}^{3} \cdot 0.01 \mathrm{~m} / \mathrm{s} \cdot 3.04 \mathrm{E}-4 \frac{\mathrm{rem}-\mathrm{m}^{2}}{\text { day }-\mu \mathrm{Ci}-\mathrm{MeV}} \cdot 0.596 \mathrm{MeV} \cdot$ 6 people $/ \mathrm{km}^{2} \cdot 1.07 \mathrm{E}+3 \mathrm{~m}^{2} \cdot\left[0.63 \frac{1-\exp \left(-1.83 \mathrm{E}+4 \mathrm{~d} \cdot 3.16 \mathrm{E}-3 \mathrm{~d}^{-1}\right)}{3.16 \mathrm{E}-3 \mathrm{~d}^{-1}}+0.37 \frac{1-\exp \left(-1.83 \mathrm{E}+4 \mathrm{~d} \cdot 8.40 \mathrm{E}-5 \mathrm{~d}^{-1}\right)}{8.40 \mathrm{E}-5 \mathrm{~d}^{-1}}\right] \cdot\left[\frac{1 \mathrm{~km}}{1000 \mathrm{~m}}\right]^{2}$

Dose $=7.32 \mathrm{E}-8$ person-rem

吉 Total Groundshine Dose $=6.23 \mathrm{E}-8$ person-rem $+7.32 \mathrm{E}-8$ person-rem $=1.35 \mathrm{E}-7$ person-rem 


\subsubsection{Inhalation Pathway}

$$
\begin{aligned}
& \text { Dose }=\mathrm{INV} \cdot \mathrm{RATE} \cdot \mathrm{DIST} \cdot \mathrm{P}_{\mathrm{sc}} \cdot \mathrm{RF} \cdot \mathrm{F}_{\mathrm{a}} \cdot \mathrm{F}_{\mathrm{r}} \cdot \mathrm{PD} \cdot \mathrm{BR} \cdot \mathrm{DCF}_{\mathrm{inh}} \cdot \mathrm{IF} \cdot\left(\frac{1 \mathrm{~km}}{1000 \mathrm{~m}}\right)^{2} \\
& \mathrm{IF}=\mathrm{AREA}_{2} \cdot \frac{\mathrm{DF}_{1}+\mathrm{DF}_{2}-\mathrm{AREA}_{1} \cdot \mathrm{SLOPE}}{2} \\
& \mathrm{SLOPE}=\frac{\mathrm{DF}_{2}-\mathrm{DF}_{1}}{\mathrm{AREA}_{2}-\mathrm{AREA}_{1}}
\end{aligned}
$$

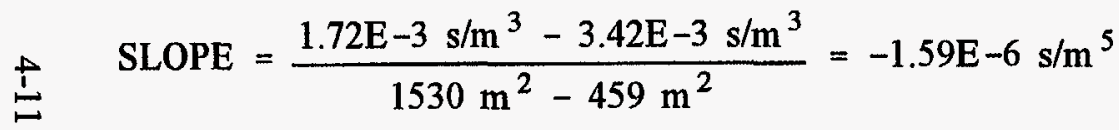

IF $=1530 \mathrm{~m}^{2} \cdot \frac{3.42 \mathrm{E}-3 \mathrm{~s} / \mathrm{m}^{3}+1.72 \mathrm{E}-3 \mathrm{~s} / \mathrm{m}^{3}-\left(459 \mathrm{~m}^{2} \cdot-1.59 \mathrm{E}-6 \mathrm{~s} / \mathrm{m}^{5}\right)}{2}=4.49 \mathrm{~s} / \mathrm{m}$

Dose $=10^{-6} \mathrm{Ci} \cdot 1$ accident $/ \mathrm{km} \cdot 1 \mathrm{~km} \cdot 1.0 \cdot 1.0 \cdot 1.0 \cdot 1.0 \cdot 6 \mathrm{people} / \mathrm{km}^{2} \cdot 3.3 \mathrm{E}-4 \mathrm{~m}^{3} / \mathrm{s} \cdot 3.2 \mathrm{E}+4 \mathrm{rem} / \mathrm{Ci} \cdot 4.49 \mathrm{~s} / \mathrm{m} \cdot\left[\frac{1 \mathrm{~km}}{1000 \mathrm{~m}}\right]^{2}$

Dose $=2.84 \mathrm{E}-10$ person $-\mathrm{rem}$ 


\subsubsection{Resuspension Pathway}

Dose $=$ Inhalation Dose $\cdot($ Resuspension Factor -1$)$

Dose $=2.84 \mathrm{E}-10$ person $-\mathrm{rem} \cdot(5.41-1)=1.25 \mathrm{E}-9$ person - rem 


\subsubsection{Cloudshine Pathway}

$$
\begin{aligned}
& \text { Dose }=\mathrm{INV} \cdot \mathrm{RATE} \cdot \mathrm{DIST} \cdot \mathrm{P}_{\mathrm{sc}} \cdot \mathrm{RF} \cdot \mathrm{F}_{\mathrm{a}} \cdot \mathrm{PD} \cdot \mathrm{DCF}_{\mathrm{imm}} \cdot \mathrm{IF} \cdot\left(\frac{1 \mathrm{~km}}{1000 \mathrm{~m}}\right)^{2} \\
& \mathrm{IF}=\mathrm{AREA}_{2} \cdot \frac{\mathrm{DF}_{1}+\mathrm{DF}_{2}-\mathrm{AREA}_{1} \cdot \mathrm{SLOPE}}{2} \\
& \mathrm{SLOPE}=\frac{\mathrm{DF}_{2}-\mathrm{DF}_{1}}{\mathrm{AREA}_{2}-\mathrm{AREA}_{1}}
\end{aligned}
$$

$\stackrel{f}{\stackrel{\omega}{\omega}} \quad$ SLOPE $=\frac{1.72 \mathrm{E}-3 \mathrm{~s} / \mathrm{m}^{3}-3.42 \mathrm{E}-3 \mathrm{~s} / \mathrm{m}^{3}}{1530 \mathrm{~m}^{2}-459 \mathrm{~m}^{2}}=-1.59 \mathrm{E}-6 \mathrm{~s} / \mathrm{m}^{5}$

$\mathrm{IF}=1530 \mathrm{~m}^{2} \cdot \frac{3.42 \mathrm{E}-3 \mathrm{~s} / \mathrm{m}^{3}+1.72 \mathrm{E}-3 \mathrm{~s} / \mathrm{m}^{3}-\left(459 \mathrm{~m}^{2} \cdot-1.59 \mathrm{E}-6 \mathrm{~s} / \mathrm{m}^{5}\right)}{2}=4.49 \mathrm{~s} / \mathrm{m}$

Dose $=10^{-6} \mathrm{Ci} \cdot 1$ accident $/ \mathrm{km} \cdot 1 \mathrm{~km} \cdot 1.0 \cdot 1.0 \cdot 1.0 \cdot 6$ people $/ \mathrm{km}^{2} \cdot 9.71 \mathrm{E}-2 \frac{\mathrm{rem}-\mathrm{m}^{3}}{\mathrm{Ci}-\mathrm{s}} \cdot 4.49 \mathrm{~s} / \mathrm{m} \cdot\left(\frac{1 \mathrm{~km}}{1000 \mathrm{~m}}\right)^{2}$

Dose $=2.62 \mathrm{E}-12$ person-rem 

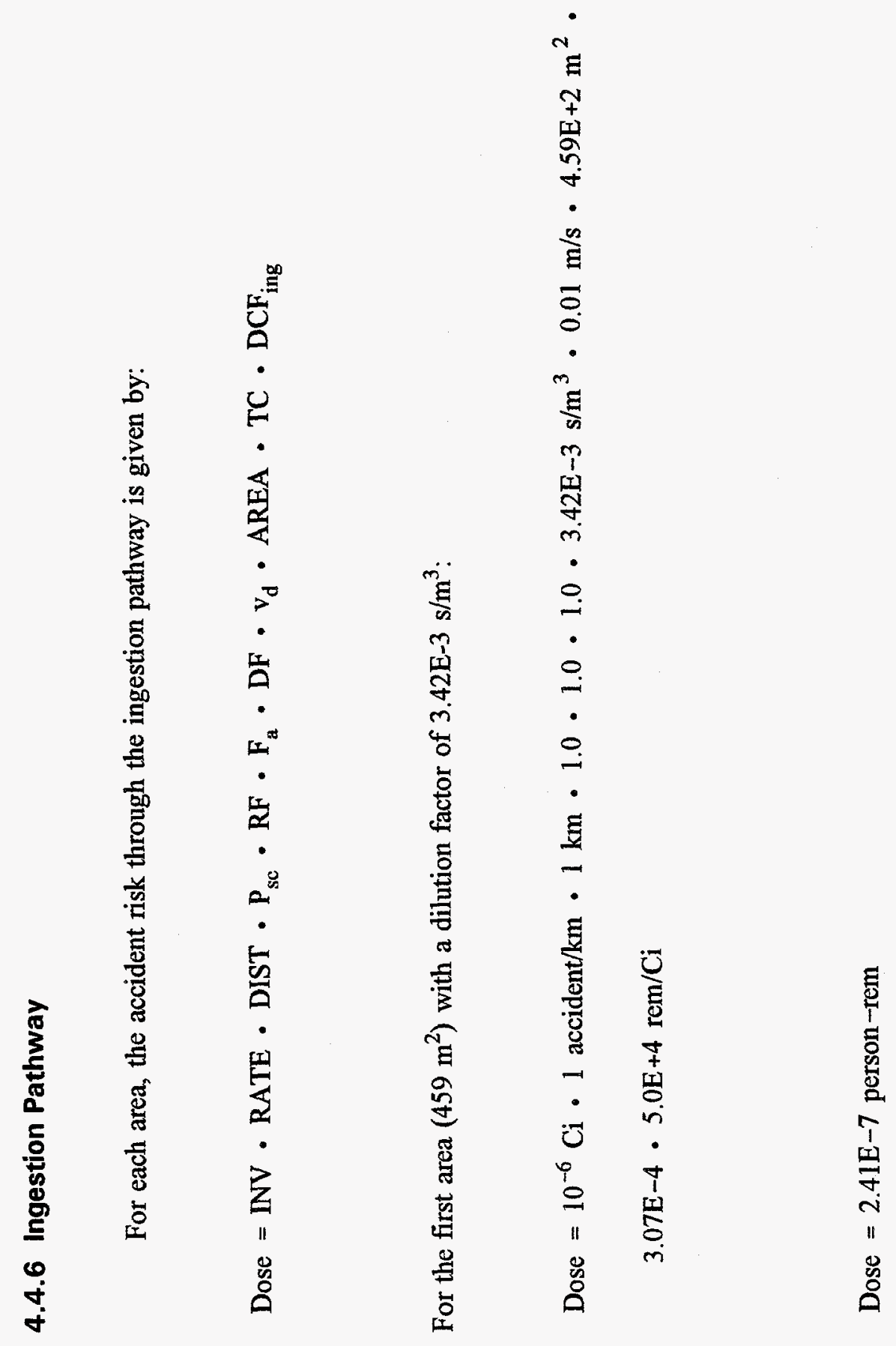

4-14 
For the second area $\left(1071 \mathrm{~m}^{2}=1530 \mathrm{~m}^{2}-459 \mathrm{~m}^{2}\right)$ with a dilution factor of $1.72 \mathrm{E}-3 \mathrm{~s} / \mathrm{m}^{3}$ :

Dose $=10^{-6} \mathrm{Ci} \cdot 1$ accident $/ \mathrm{km} \cdot 1 \mathrm{~km} \cdot 1.0 \cdot 1.0 \cdot 1.0 \cdot 1.72 \mathrm{E}-3 \mathrm{~s} / \mathrm{m}^{3} \cdot 0.01 \mathrm{~m} / \mathrm{s} \cdot 1.07 \mathrm{E}+3 \mathrm{~m}^{2}$. $3.07 \mathrm{E}-4 \cdot 5.0 \mathrm{E}+4 \mathrm{rem} / \mathrm{Ci}$

Dose $=2.83 \mathrm{E}-7$ person-rem

$\stackrel{f}{+}$ Total Ingestion Dose $=2.41 \mathrm{E}-7$ person-rem $+2.83 \mathrm{E}-7$ person $-\mathrm{rem}=5.24 \mathrm{E}-7$ person - rem 


\section{VALIDATION OF THE INCIDENT-FREE RADIATION DOSES CALCULATED USING THE RISKIND COMPUTER CODE}

\subsection{Validation Analysis}

The RISKIND computer code (Yuan et al. 1993) was used to estimate incident-free radiation doses for maximally exposed individuals for the transportation analyses in DOE (1995).

The purpose of this analysis was to validate the incident-free radiation doses for maximally exposed individuals calculated by RISKIND by comparing them to hand-calculated values using identical data and equations. The definition of validation used in this analysis was "the test and evaluation of the completed software to ensure compliance with software requirements" (ASME 1989). In the context of this analysis, compliance with software requirements means that the differences between the radiation doses calculated by RISKIND and the hand-calculated radiation doses should be small (i.e., within the acceptance criterion established for the validation).

The acceptance criterion for this validation was a percent difference of 5 percent. If the RISKIND calculated radiation doses differed from the hand-calculated radiation doses by more than 5 percent, then the result was considered unacceptable. If the RISKIND calculated radiation doses differed from the hand-calculated radiation doses by less than 5 percent, then the result was considered acceptable.

Two types of incident-free maximally exposed individual scenarios were evaluated in DOE (1995): (1) occupational scenarios and (2) general population scenarios. Two transport modes were also evaluated in DOE (1995): (1) truck and (2) rail. The equations used to calculate incident-free radiation doses are contained in Yuan et al. (1993); the output from the RISKIND computer code for these scenarios is contained in Appendix C. Occupational doses for truck shipments were evaluated using hand calculations and a radiation dose rate of $2 \mathrm{mrem} / \mathrm{hr}$ in the truck cab; RISKIND was not used. Therefore, occupational doses for truck shipments were not included in this validation analysis.

Occupational doses for rail shipments were calculated using a radiation dose rate of $21.1 \mathrm{mrem} / \mathrm{hr}$ at 1 meter (3.25 feet) from the shipping container; this dose rate yields a dose rate of 
$10 \mathrm{mrem} / \mathrm{hr}$ at 2 meters (6.5 feet) from the shipping container, the regulatory limit. The rail worker was an individual in a railyard who spent a time- and distance-weighted average of 0.16 hours inspecting, classifying, and repairing railcars (Wooden 1986). Radiation doses for this scenario were calculated using the equation:

Dose $=$ Dose rate $\cdot$ Time

Dose $=21.1 \mathrm{mrem} / \mathrm{hr} \cdot 0.16$ hour $\cdot \frac{1 \mathrm{rem}}{1000 \mathrm{mrem}}=3.38 \mathrm{E}-03 \mathrm{rem}$

General population doses for truck and rail shipments were also calculated using a radiation dose rate of $21.1 \mathrm{mrem} / \mathrm{hr}$ at 1 meter (3.25 feet) from the shipping container; this dose rate yields a dose rate of $10 \mathrm{mrem} / \mathrm{hr}$ at 2 meters (6.5 feet) from the shipping container, the regulatory limit. For truck shipments, the largest radiation dose for the maximally exposed individual was for a service station attendant working at a distance of 20 meters (65 feet) from the shipping container for 2 hours. Radiation doses for this scenario were calculated using the equation:

Dose $=$ Dose rate $\cdot$ Time

Normalized dose rate $=A_{0} \cdot \frac{1}{r^{2}}+A_{1} \cdot \frac{1}{r}+A_{2} \quad$ for $10 m<r \leq 2000 m$

Normalized dose rate $=3.79031 \mathrm{E}+01 \cdot \frac{1}{20^{2}}+1.09056 \mathrm{E}-01 \cdot \frac{1}{20}+-2.44353 \mathrm{E}-03$

Normalized dose rate $=0.0978 \mathrm{mrem} / \mathrm{hr}$

Dose rate $=$ normalized dose rate $\cdot \frac{21.1 \mathrm{mrem} / \mathrm{hr}}{10 \mathrm{mrem} / \mathrm{hr}}=0.0978 \mathrm{mrem} / \mathrm{hr} \cdot \frac{21.1 \mathrm{mrem} / \mathrm{hr}}{10 \mathrm{mrem} / \mathrm{hr}}$

Dose rate $=0.206 \mathrm{mrem} / \mathrm{hr}$

Dose $=0.206 \mathrm{mrem} / \mathrm{hr} \cdot 2 \mathrm{hour} \cdot \frac{1 \mathrm{rem}}{1000 \mathrm{mrem}}=4.13 \mathrm{E}-04 \mathrm{rem}$ 
For rail shipments, the largest radiation dose for the maximally exposed individual was for a railyard worker working at a distance of $10 \mathrm{~m}$ (32.5 feet) from the shipping container for 2 hours. Radiation doses for this scenario were calculated using the equation:

Dose $=$ Dose rate $\cdot$ Time

Normalized dose rate $=A_{0} \cdot \frac{1}{r}+A_{1}+A_{2} \cdot r \quad$ for $1 \mathrm{~m} \leq \mathrm{r} \leq 10 \mathrm{~m}$

Normalized dose rate $=1.03586 \mathrm{E}+01 \cdot \frac{1}{10}+-9.70747 \mathrm{E}-01+3.18380 \mathrm{E}-02 \cdot 10$

Normalized dose rate $=0.383 \mathrm{mrem} / \mathrm{hr}$

Dose rate $=$ Normalized dose rate $\cdot \frac{21.1 \mathrm{mrem} / \mathrm{hr}}{10 \mathrm{mrem} / \mathrm{hr}}=0.383 \mathrm{mrem} / \mathrm{hr} \cdot \frac{21.1 \mathrm{mrem} / \mathrm{hr}}{10 \mathrm{mrem} / \mathrm{hr}}$

Dose rate $=0.808 \mathrm{mrem} / \mathrm{hr}$

Dose $=0.808 \mathrm{mrem} / \mathrm{hr} \cdot 2 \mathrm{hour} \cdot \frac{1 \mathrm{rem}}{1000 \mathrm{mrem}}=1.62 \mathrm{E}-03 \mathrm{rem}$

Table 5-1 provides the radiation doses calculated by the RISKIND computer code, the radiation doses estimated using hand calculations, and the percent differences between the RISKINDand hand-calculated radiation doses.

\subsection{Results of Validation Analysis}

The minimum percent difference observed in Table 5-1 was zero, which meant that RISKIND and the hand calculations yielded identical results. The maximum percent difference observed in Table 5-1 was 3.1 percent, within the 5 percent validation criterion. These results show that the differences between the radiation doses estimated by RISKIND and the radiation doses estimated using hand calculations are small and are within the acceptance criterion established for the validation. Therefore, the incident-free radiation doses generated using the RISKIND computer code are considered validated. 
Table 5-1. RISKIND-calculated and hand-calculated incident-free radiation doses.

\begin{tabular}{llll}
\hline & \multicolumn{2}{c}{ Radiation dose (rem) } & \\
\cline { 2 - 3 } Scenario & RISKIND & Hand-calculated & Percent difference \\
\hline Rail - occupational & $3.38 \mathrm{E}-3$ & $3.38 \mathrm{E}-3$ & 0.0 \\
$\begin{array}{l}\text { Truck - general } \\
\text { population }\end{array}$ & $4.07 \mathrm{E}-4$ & $4.13 \mathrm{E}-4$ & -1.5 \\
$\begin{array}{l}\text { Train - general } \\
\text { population }\end{array}$ & $1.67 \mathrm{E}-3$ & $1.62 \mathrm{E}-3$ & 3.1 \\
\hline
\end{tabular}




\section{VALIDATION OF THE ACCIDENT RADIATION DOSES CALCULATED USING THE RISKIND COMPUTER CODE}

\subsection{Validation Analysis}

The RISKIND computer code (Yuan et al. 1993) was used to estimate the consequences from reasonably foreseeable transportation accidents for the transportation analyses in DOE (1995).

The purpose of this analysis was to validate the accident consequences (radiation doses) for maximally exposed individuals calculated by RISKIND by comparing them to hand-calculated values using identical data and equations. The definition of validation used in this analysis was "the test and evaluation of the completed software to ensure compliance with software requirements" (ASME 1989). In the context of this analysis, compliance with software requirements means that the differences between the radiation doses calculated by RISKIND and the hand-calculated radiation doses should be small (i.e., within the acceptance criterion established for the validation).

The acceptance criterion for this validation was a percent difference of 5 percent. If the RISKIND calculated radiation doses differed from the hand-calculated radiation doses by more than 5 percent, then the result was considered unacceptable. If the RISKIND calculated radiation doses differed from the hand-calculated radiation doses by less than 5 percent, then the result was considered acceptable.

A simple case was constructed for this validation analysis. One radionuclide, cesium-137, was used in the validation analysis and radiation doses were estimated through the groundshine, cloudshine, inhalation, and ingestion pathways. The radiation doses calculated by the RISKIND computer code are listed in Table 6-1. Appendix D contains the RISKIND output.

The equations used to calculate the radiation doses are listed in Yuan et al. (1993). The radiation doses calculated using hand calculations are listed in Table 6-1. Section 6.4 contains the detailed hand calculations used to calculate these radiation doses. Table 6-l also contains the percent differences between the RISKIND and the hand calculated radiation doses. 
Table 6-1. RISKIND-calculated and hand-calculated radiation doses.

\begin{tabular}{llll}
\hline & \multicolumn{2}{c}{ Radiation dose (rem) } & \\
\cline { 2 - 3 } \multicolumn{1}{c}{ Pathway } & RISKIND & Hand-calculated & Percent difference \\
\hline Short term & $2.29 \mathrm{E}-1$ & $2.30 \mathrm{E}-1$ & -0.43 \\
Long term & & & \\
Groundshine & $5.16 \mathrm{E}-1$ & $5.25 \mathrm{E}-1$ & -1.7 \\
Cloudshine & $7.99 \mathrm{E}-5$ & $7.97 \mathrm{E}-5$ & 0.25 \\
Inhalation & $7.60 \mathrm{E}-3$ & $7.51 \mathrm{E}-3$ & 1.2 \\
Ingestion & & & \\
Vegetables & $4.68 \mathrm{E}-1$ & $4.69 \mathrm{E}-1$ & -0.21 \\
Meat & $7.87 \mathrm{E}-2$ & $7.90 \mathrm{E}-2$ & -0.38 \\
Milk & $6.69 \mathrm{E}-1$ & $6.69 \mathrm{E}-1$ & 0.0 \\
\hline
\end{tabular}

\subsection{Results of Validation Analysis}

The minimum percent difference observed in Table 6-1 was zero, which meant that RISKIND and the hand calculation yielded identical results. The maximum percent difference observed in Table 6-1 was 1.7 percent, well within the 5 percent validation criterion. These results show that the differences between the radiation doses estimated by RISKIND and the radiation doses estimated using hand calculations are small and are within the acceptance criterion established for the validation. Therefore, the radiation doses generated using the RISKIND computer code are considered validated. 


\subsection{Detailed Hand Calculations for the Transportation Accident Scenario}

This section gives hand calculations for the following: atmospheric concentration at the receptor, short-term radiation doses, and long-term radiation doses. It also includes a list of symbols used in the calculations.

\subsubsection{List of Symbols}

BR = Breathing rate $\left(2.5 \mathrm{E}-4 \mathrm{~m}^{3} / \mathrm{s}\right.$ for short-term doses, $3.5 \mathrm{E}-4 \mathrm{~m}^{3} / \mathrm{s}$ for long-term doses)

$\mathrm{B}_{\mathrm{v}} \quad=\quad$ Concentration ratio (wet weight) $(0.10)$

$\chi \quad=\quad$ Time-integrated atmospheric concentration $\left(2.04 \mathrm{E}-2 \mathrm{Ci}-\mathrm{s} / \mathrm{m}^{3}\right)$

D $\quad=\quad$ Decontamination factor for vegetables (0.5)

$\mathrm{DCF}_{\mathrm{cld}} \quad=\quad$ Cloudshine dose conversion factor $\left(2.86 \mathrm{E}-6 \mathrm{rem}-\mathrm{m}^{3} / \mathrm{pCi}-\mathrm{yr}\right)$

$\mathrm{DCF}_{\mathrm{grd}} \quad=\quad$ Groundshine dose conversion factor $\left(5.82 \mathrm{E}-8 \mathrm{rem}-\mathrm{m}^{2} / \mathrm{pCi}-\mathrm{yr}\right)$

$\mathrm{DCF}_{\text {ing }} \quad=\quad$ Ingestion dose conversion factor $(5.00 \mathrm{E}-8 \mathrm{rem} / \mathrm{pCi})$

$\mathrm{DCF}_{\text {inh }} \quad=\quad$ Inhalation dose conversion factor $(3.19 \mathrm{E}-8 \mathrm{rem} / \mathrm{pCi})$

$\mathrm{F}_{\mathrm{b}} \quad=\quad$ Beef transfer coefficient $(0.004 \mathrm{~d} / \mathrm{kg})$

$\mathrm{F}_{\mathrm{m}} \quad=\quad$ Milk transfer coefficient $(0.012 \mathrm{~d} / \mathrm{L})$

FG $\quad=\quad$ Fraction of beef and milk from home garden $(0.5)$

$\mathrm{H} \quad$ = Effective release height $(10 \mathrm{~m})$

$\lambda_{\mathrm{i}} \quad=\quad$ Decay constant $\left(2.30 \mathrm{E}-2 \mathrm{yr}^{-1}\right)$

$\lambda_{\mathrm{g}} \quad=\quad$ Environmental loss rate constant $\left(0.0139 \mathrm{yr}^{-1}\right)$

$\lambda_{\mathrm{r}} \quad=\quad$ Resuspension factor decay constant $\left(5.06 \mathrm{yr}^{-1}\right)$

$\lambda_{\mathrm{w}} \quad=\quad$ Weathering rate constant $\left(0.0495 \mathrm{~d}^{-1}\right)$

P = Surface soil density (dry weight) $\left(240 \mathrm{~kg} / \mathrm{m}^{2}\right)$

Q $\quad=\quad$ Release $(77 \mathrm{Ci})$

$\mathrm{Q}_{\text {for }} \quad=\quad$ Feed consumption rate $(50 \mathrm{~kg} / \mathrm{d})$

I $\quad=\quad$ Interception fraction (0.2)

$\mathrm{R} \quad=\quad$ Resuspension factor $\left(1.0 \mathrm{E}-5 \mathrm{~m}^{-1}\right)$

$\mathrm{SF}_{\mathrm{cld}} \quad=\quad$ Shielding factor for cloudshine doses $(0.70)$

$\mathrm{SF}_{\mathrm{grd}} \quad=\quad$ Shielding factor for groundshine doses $(0.45)$ 


$\begin{array}{lll}\mathrm{SF}_{\text {inh }} & = & \text { Shielding factor for inhalation doses }(0.75) \\ \sigma_{\mathrm{y}} & = & \text { Standard deviation in y direction } \\ \sigma_{\mathrm{z}} & = & \text { Standard deviation in z direction } \\ \mathrm{t}_{\text {for }} & = & \text { Forage grow time }(30 \mathrm{~d}) \\ \mathrm{t}_{\text {veg }} & = & \text { Vegetable grow time }(60 \mathrm{~d}) \\ \mathrm{T} & = & \text { Long-term build-up time }(1 \mathrm{yr}) \\ \mathrm{T}_{\text {grd }} & = & \text { Short-term groundshine exposure time } \\ \mathrm{TF} & = & \text { Translocation factor }(1.0) \\ \mathrm{u} & = & \text { Wind speed }(4 \mathrm{~m} / \mathrm{s}) \\ \mathrm{U}_{\mathrm{b}} & = & \text { Beef consumption rate }(0.30 \mathrm{~kg} / \mathrm{d}) \\ \mathrm{U}_{\mathrm{m}} & = & \text { Milk consumption rate }(0.85 \mathrm{~L} / \mathrm{d}) \\ \mathrm{U}_{\mathrm{v}} & = & \text { Vegetable consumption rate }(0.77 \mathrm{~kg} / \mathrm{d}) \\ \mathrm{v}_{\mathrm{d}} & = & \text { Deposition velocity on soil }(0.001 \mathrm{~m} / \mathrm{s}) \\ \mathrm{v}_{\mathrm{d}}(\text { soil }) & = & \text { Deposition velocity on soil }(0.001 \mathrm{~m} / \mathrm{s}) \\ \mathrm{v}_{\mathrm{d}}(\text { for }) & = & \text { Deposition velocity on forage }(0.01 \mathrm{~m} / \mathrm{s}) \\ \mathrm{x} & = & \text { Distance in x-direction to receptor }(130 \mathrm{~m}) \\ \mathrm{y} & = & \text { Crosswind distance to receptor }(0 \mathrm{~m}) \\ \mathrm{Y}_{\text {for }} & = & \text { Forage yield }\left(0.75 \mathrm{~kg} / \mathrm{m}^{2}\right) \\ \mathrm{Y}_{\mathrm{veg}} & = & \text { Vegetable yield }\left(2.0 \mathrm{~kg} / \mathrm{m}^{2}\right)\end{array}$

Fitted parameters for calculating $\sigma_{\mathrm{y}}$ and $\sigma_{\mathrm{z}}$ :

$\begin{array}{lll}\sigma_{\theta} & =10 \\ \mathrm{a} & =0.222 \\ \mathrm{~b} & =0.725 \\ \mathrm{c} & =-1.7\end{array}$




\subsubsection{Atmospheric Concentration at Receptor}

The RISKIND computer code calculates the atmospheric concentration of a radionuclide at the location of the receptor using the Gaussian plume atmospheric dispersion model:

$$
\begin{aligned}
& \chi=\frac{\mathrm{Q}}{\pi \cdot \sigma_{\mathrm{y}} \cdot \sigma_{\mathrm{z}} \cdot \mathrm{u}} \cdot \exp \left[-\left[\frac{\mathrm{y}^{2}}{2 \sigma_{\mathrm{y}}^{2}}+\frac{\mathrm{H}^{2}}{2 \sigma_{z}^{2}}\right]\right] \\
& \sigma_{\mathrm{y}}(\mathrm{x})=\left(0.000246 \cdot \sigma_{\theta}^{2}+0.00576 \cdot \sigma_{\theta}+0.066\right) \cdot \mathrm{x}^{0.9031} \\
& \sigma_{z}(\mathrm{x})=\mathrm{a} \cdot \mathrm{x}^{\mathrm{b}}+\mathrm{c}
\end{aligned}
$$

From Yuan et al. (1993), at a distance of $130 \mathrm{~m}$ for class D stability:

$$
\begin{aligned}
Q & =77 \mathrm{Ci} \\
\mathbf{x} & =130 \mathrm{~m} \\
\sigma_{\theta} & =10 \\
\mathrm{a} & =0.222 \\
\mathrm{~b} & =0.725 \\
\mathrm{c} & =-1.7 \\
\mathbf{u} & =4 \mathrm{~m} / \mathrm{s} \\
\mathrm{y} & =0 \mathrm{~m} \\
\mathrm{H} & =10 \mathrm{~m} \\
\sigma_{\mathrm{y}}(130 \mathrm{~m}) & =\left(0.000246 \cdot 10^{2}+0.00576 \cdot 10+0.066\right) \cdot 130^{0.9031}=12.0 \mathrm{~m} \\
\sigma_{\mathrm{z}}(130 \mathrm{~m})= & 0.222 \cdot 130^{0.725}+-1.7=5.87 \mathrm{~m}
\end{aligned}
$$




$$
\begin{aligned}
& \chi=\frac{77 \mathrm{Ci}}{\pi \cdot 12.0 \mathrm{~m} \cdot 5.87 \mathrm{~m} \cdot 4 \mathrm{~m} / \mathrm{s}} \cdot \exp \left[-\left(\frac{(0 \mathrm{~m})^{2}}{2 \cdot(12.0 \mathrm{~m})^{2}}+\frac{(10 \mathrm{~m})^{2}}{2 \cdot(5.87 \mathrm{~m})^{2}}\right]\right] \\
& \chi=2.04 \mathrm{E}-2 \mathrm{Ci}-\mathrm{s} / \mathrm{m}^{3}
\end{aligned}
$$

\subsubsection{Short-Term Radiation Doses}

The short-term radiation dose calculated by RISKIND has three components:

(1) groundshine, (2) cloudshine, and (3) inhalation.

\subsubsection{Short-Term Groundshine Radiation Dose.}

Dose $=\chi \cdot \mathrm{v}_{\mathrm{d}} \cdot \mathrm{DCF}_{\mathrm{grd}} \cdot \mathrm{T}_{\mathrm{grd}}$

Dose $=2.04 \mathrm{E}-2 \mathrm{Ci}-\mathrm{s} / \mathrm{m}^{3} \cdot 0.001 \mathrm{~m} / \mathrm{s} \cdot 5.82 \mathrm{E}-8 \frac{\mathrm{rem}-\mathrm{m}^{2}}{\mathrm{pCi}-\mathrm{yr}} \cdot 2 \mathrm{hr} \cdot \frac{1 \mathrm{yr}}{8760 \mathrm{hr}} \cdot \frac{10^{12} \mathrm{pCi}}{1 \mathrm{Ci}}$

Dose $=2.71 \mathrm{E}-4 \mathrm{rem}$

\subsubsection{Short-Term Cloudshine Radiation Dose.}

Dose $=\chi \cdot \mathrm{DCF}_{\mathrm{cld}}$

Dose $=2.04 \mathrm{E}-2 \mathrm{Ci}-\mathrm{s} / \mathrm{m}^{3} \cdot 2.86 \mathrm{E}-6 \frac{\mathrm{rem}-\mathrm{m}^{3}}{\mathrm{pCi}-\mathrm{yr}} \cdot \frac{1 \mathrm{yr}}{3.154 \mathrm{E}+7 \mathrm{~s}} \cdot \frac{10^{12} \mathrm{pCi}}{1 \mathrm{Ci}}$

Dose $=1.85 \mathrm{E}-3$ rem 


\subsubsection{Short-Term Inhalation Radiation Dose.}

Dose $=\chi \cdot \mathrm{BR} \cdot \mathrm{DCF}_{\mathrm{inh}}$

Dose $=2.04 \mathrm{E}-2 \mathrm{Ci}-\mathrm{s} / \mathrm{m}^{3} \cdot 3.5 \mathrm{E}-4 \mathrm{~m}^{3} / \mathrm{s} \cdot 3.19 \mathrm{E}-8 \mathrm{rem} / \mathrm{pCi} \cdot \frac{10^{12} \mathrm{pCi}}{1 \mathrm{Ci}}$

Dose $=2.28 \mathrm{E}-1$ rem

6.3.3.4 Total Short-Term Radiation Dose.

Dose $=$ Dose $_{\text {grd }}+$ Dose $_{\text {cld }}+$ Dose $_{\text {inh }}$

$2.71 \mathrm{E}-4 \mathrm{rem}+1.85 \mathrm{E}-3 \mathrm{rem}+2.28 \mathrm{E}-1 \mathrm{rem}=2.30 \mathrm{E}-1 \mathrm{rem}$ 


\subsubsection{Long-Term Radiation Doses}

The long-term radiation dose calculated by RISKIND has four components: (1) groundshine, (2) cloudshine, (3) inhalation, and (4) ingestion.

\subsubsection{Long-Term Groundshine Radiation Dose.}

Dose $=\chi \cdot \mathrm{v}_{\mathrm{d}} \cdot \frac{1-\exp \left[-\left(\lambda_{\mathrm{i}}+\lambda_{\mathrm{g}}\right) \cdot \mathrm{T}\right]}{\lambda_{\mathrm{i}}+\lambda_{\mathrm{g}}} \cdot \mathrm{DCF}_{\mathrm{grd}} \cdot \mathrm{SF}_{\mathrm{grd}}$

Dose $=2.04 \mathrm{E}-2 \mathrm{Ci}-\mathrm{s} / \mathrm{m}^{3} \cdot 0.001 \mathrm{~m} / \mathrm{s} \cdot \frac{1-\exp \left[-\left(2.30 \mathrm{E}-2 \mathrm{yr}^{-1}+0.0139 \mathrm{yr}^{-1}\right) \cdot 1 \mathrm{yr}\right]}{2.30 \mathrm{E}-2 \mathrm{yr}^{-1}+0.0139 \mathrm{yr}^{-1}} \cdot$

$5.82 \mathrm{E}-8 \frac{\mathrm{rem}-\mathrm{m}^{2}}{\mathrm{pCi}-\mathrm{yr}} \cdot \frac{10^{12} \mathrm{pCi}}{1 \mathrm{Ci}} \cdot 0.45$

Dose $=5.25 \mathrm{E}-1 \mathrm{rem}$

\subsubsection{Long-Term Cloudshine Radiation Dose.}

Dose $=\chi \cdot \mathrm{v}_{\mathrm{d}} \cdot \frac{1-\exp \left[-\left(\lambda_{\mathrm{i}}+\lambda_{\mathrm{g}}+\lambda_{\mathrm{r}}\right) \cdot \mathrm{T}\right]}{\lambda_{\mathrm{i}}+\lambda_{\mathrm{g}}+\lambda_{\mathrm{r}}} \cdot \mathrm{R} \cdot \mathrm{DCF}_{\mathrm{cld}} \cdot \mathrm{SF}_{\mathrm{cld}}$

Dose $=2.04 \mathrm{E}-2 \mathrm{Ci}-\mathrm{s} / \mathrm{m}^{3} \cdot 0.001 \mathrm{~m} / \mathrm{s} \cdot \frac{1-\exp \left[-\left(2.30 \mathrm{E}-2 \mathrm{yr}^{-1}+0.0139 \mathrm{yr}^{-1}+5.06 \mathrm{yr}^{-1}\right) \cdot 1 \mathrm{yr}\right]}{2.30 \mathrm{E}-2 \mathrm{yr}^{-1}+0.0139 \mathrm{yr}^{-1}+5.06 \mathrm{yr}^{-1}} \cdot$

$$
1.0 \mathrm{E}-5 \mathrm{~m}^{-1} \cdot 2.86 \mathrm{E}-6 \frac{\mathrm{rem}-\mathrm{m}^{3}}{\mathrm{pCi}-\mathrm{yr}} \cdot \frac{10^{12} \mathrm{pCi}}{1 \mathrm{Ci}} \cdot 0.70
$$

Dose $=7.97 \mathrm{E}-5$ rem 


\subsubsection{Long-Term Inhalation Radiation Dose.}

$$
\begin{aligned}
\text { Dose } & =\chi \cdot \mathrm{v}_{\mathrm{d}} \cdot \frac{1-\exp \left[-\left(\lambda_{\mathrm{i}}+\lambda_{\mathrm{g}}+\lambda_{\mathrm{r}}\right) \cdot \mathrm{T}\right]}{\lambda_{\mathrm{i}}+\lambda_{\mathrm{g}}+\lambda_{\mathrm{r}}} \cdot \mathrm{R} \cdot \mathrm{BR} \cdot \mathrm{DCF}_{\mathrm{inh}} \cdot \mathrm{SF}_{\mathrm{inh}} \\
\text { Dose } & =2.04 \mathrm{E}-2 \mathrm{Ci}-\mathrm{s}_{\mathrm{m}}^{3} \cdot 0.001 \mathrm{~m} / \mathrm{s} \cdot \frac{1-\exp \left[-\left(2.30 \mathrm{E}-2 \mathrm{yr}^{-1}+0.0139 \mathrm{yr}^{-1}+5.06 \mathrm{yr}^{-1}\right) \cdot 1 \mathrm{yr}\right]}{2.30 \mathrm{E}-2 \mathrm{yr}^{-1}+0.0139 \mathrm{yr}^{-1}+5.06 \mathrm{yr}^{-1}} \\
& 1.0 \mathrm{E}-5 \mathrm{~m}^{-1} \cdot 2.5 \mathrm{E}-4 \mathrm{~m}^{3} / \mathrm{s} \cdot 3.19 \mathrm{E}-8 \mathrm{rem} / \mathrm{pCi} \cdot \frac{3.154 \mathrm{E}+7 \mathrm{~s}}{1 \mathrm{yr}} \cdot \frac{10^{12} \mathrm{pCi}}{1 \mathrm{Ci}} \cdot 0.75 \\
\text { Dose }= & 7.51 \mathrm{E}-3 \mathrm{rem}
\end{aligned}
$$

6.3.4.4 Long-Term Ingestion Radiation Dose. The long-term ingestion radiation dose calculated by RISKIND has two components: (1) the radiation dose from root uptake into plants after initial deposition on soil, and (2) the radiation dose from the deposition of resuspended radioactivity on plants after initial deposition on soil. Three pathways are considered by RISKIND: (1) vegetables, (2) meat, and (3) milk. In this validation analysis, initially contaminated food was assumed to be discarded.

\subsection{Long-Term Ingestion Radiation Dose Through the Vegetable}

Pathway-This section provides calculations on root uptake of radioactivity, deposition of resuspended radioactivity, and total long-term ingestion radiation dose. 


\subsection{Root Uptake of Radioactivity-}

$$
\begin{aligned}
\text { Dose }= & \chi \cdot \mathrm{v}_{\mathrm{d}} \text { (soil) } \cdot \frac{1-\exp \left[-\left(\lambda_{\mathrm{i}}+\lambda_{\mathrm{g}}\right) \cdot \mathrm{T}\right]}{\lambda_{\mathrm{i}}+\lambda_{\mathrm{g}}} \cdot \mathrm{B}_{\mathrm{v}} \cdot \frac{1}{\mathrm{P}} \cdot \mathrm{U}_{\mathrm{v}} \cdot \mathrm{D} \cdot \mathrm{DCF}_{\text {ing }} \\
\text { Dose }= & 2.04 \mathrm{E}-2 \mathrm{Ci}-\mathrm{s} / \mathrm{m}^{3} \cdot 0.001 \mathrm{~m} / \mathrm{s} \cdot \frac{1-\exp \left[-\left(2.30 \mathrm{E}-2 \mathrm{yr}^{-1}+0.0139 \mathrm{yr}^{-1}\right) \cdot 1 \mathrm{yr}\right]}{2.30 \mathrm{E}-2 \mathrm{yr}^{-1}+0.0139 \mathrm{yr}^{-1}} \cdot \\
& 0.010 \frac{\mathrm{pCi} / \mathrm{kg}(\text { wetveg })}{\mathrm{pCi} / \mathrm{kg}(\mathrm{dry} \mathrm{soil})} \cdot \frac{1}{240 \mathrm{~kg}(\mathrm{dry} \mathrm{soil}) / \mathrm{m}^{2}} \cdot 0.77 \mathrm{~kg} / \mathrm{d} \cdot 365 \mathrm{~d} / \mathrm{yr} \cdot \\
& 0.50 \cdot 5.00 \mathrm{E}-8 \mathrm{rem} / \mathrm{pCi} \cdot \frac{10^{12} \mathrm{pCi}}{1 \mathrm{Ci}}
\end{aligned}
$$

Dose $=5.85 \mathrm{E}-3 \mathrm{rem}$

\subsection{Deposition of Resuspended Radioactivity-}

$$
\begin{aligned}
\text { Dose }= & \chi \cdot \mathrm{v}_{\mathrm{d}} \text { (soil) } \cdot \frac{1-\exp \left[-\left(\lambda_{\mathrm{i}}+\lambda_{\mathrm{g}}+\lambda_{\mathrm{r}}\right) \cdot \mathrm{T}\right]}{\lambda_{\mathrm{i}}+\lambda_{\mathrm{g}}+\lambda_{\mathrm{r}}} \cdot \mathrm{R} \cdot \mathrm{v}_{\mathrm{d}}(\mathrm{veg}) \cdot \mathrm{r} \cdot \mathrm{TF} \cdot \\
& \frac{1-\exp \left[-\lambda_{\mathrm{w}} \cdot \mathrm{t}_{\mathrm{veg}}\right]}{\lambda_{\mathrm{w}} \cdot \mathrm{Y}_{\mathrm{veg}}} \cdot \mathrm{U}_{\mathrm{v}} \cdot \mathrm{D} \cdot \mathrm{DCF} \mathrm{Fin}_{\mathrm{ing}}
\end{aligned}
$$

Dose $=2.04 \mathrm{E}-2 \mathrm{Ci}-\mathrm{s} / \mathrm{m}^{3} \cdot 0.001 \mathrm{~m} / \mathrm{s} \cdot \frac{1-\exp \left[-\left(2.30 \mathrm{E}-2 \mathrm{yr}^{-1}+0.0139 \mathrm{yr}^{-1}+5.06 \mathrm{yr}^{-1}\right) \cdot 1 \mathrm{yr}\right]}{2.30 \mathrm{E}-2 \mathrm{yr}^{-1}+0.0139 \mathrm{yr}^{-1}+5.06 \mathrm{yr}^{-1}} \cdot$

$$
\begin{aligned}
& 1.0 \mathrm{E}-5 \mathrm{~m}^{-1} \cdot 0.01 \mathrm{~m} / \mathrm{s} \cdot 0.2 \cdot 1.0 \cdot \frac{1-\exp \left[-0.0495 \mathrm{~d}^{-1} \cdot 60 \mathrm{~d}\right]}{0.0495 \mathrm{~d}^{-1} \cdot 2.0 \mathrm{~kg} / \mathrm{m}^{2}} \cdot 0.77 \mathrm{~kg} / \mathrm{d} \cdot \\
& 0.50 \cdot 5.0 \mathrm{E}-8 \mathrm{rem} / \mathrm{pCi} \cdot \frac{3.154 \mathrm{E}+7 \mathrm{~s}}{1 \mathrm{yr}} \cdot \frac{10^{12} \mathrm{pCi}}{1 \mathrm{Ci}}
\end{aligned}
$$

Dose $=4.63 \mathrm{E}-1 \mathrm{rem}$ 
Vegetable Pathway-

Dose $=5.85 \mathrm{E}-3 \mathrm{rem}+4.63 \mathrm{E}-1 \mathrm{rem}=4.69 \mathrm{E}-1 \mathrm{rem}$

\subsection{Long-Term Ingestion Radiation Dose Through the Meat}

Pathway-This section provides calculations on root uptake of radioactivity, deposition of resuspended radioactivity, and total long-term ingestion radiation dose.

\subsection{Root Uptake of Radioactivity-}

Dose $=\chi \cdot \mathrm{v}_{\mathrm{d}}($ soil $) \cdot \frac{1-\exp \left[-\left(\lambda_{\mathrm{i}}+\lambda_{\mathrm{g}}\right) \cdot \mathrm{T}\right]}{\lambda_{\mathrm{i}}+\lambda_{\mathrm{g}}} \cdot \mathrm{B}_{\mathrm{v}} \cdot \frac{1}{\mathrm{P}} \cdot \mathrm{Q}_{\mathrm{for}} \cdot \mathrm{F}_{\mathrm{b}} \cdot \mathrm{FG} \cdot \mathrm{U}_{\mathrm{b}} \cdot \mathrm{DCF}_{\text {ing }}$

Dose $=2.04 \mathrm{E}-2 \mathrm{Ci}-\mathrm{s} / \mathrm{m}^{3} \cdot 0.001 \mathrm{~m} / \mathrm{s} \cdot \frac{1-\exp \left[-\left(2.30 \mathrm{E}-2 \mathrm{yr}^{-1}+0.0139 \mathrm{yr}^{-1}\right) \cdot 1 \mathrm{yr}\right]}{2.30 \mathrm{E}-2 \mathrm{yr}^{-1}+0.0139 \mathrm{yr}^{-1}} \cdot$

$0.010 \frac{\mathrm{pCi} / \mathrm{kg} \text { (wetveg) }}{\mathrm{pCi} / \mathrm{kg} \text { (dry soil) }} \cdot \frac{1}{240 \mathrm{~kg}(\text { dry soil }) / \mathrm{m}^{2}} \cdot 50 \mathrm{~kg} / \mathrm{d} \cdot 0.004 \mathrm{~d} / \mathrm{kg} \cdot 0.50$

$0.30 \mathrm{~kg} / \mathrm{d} \cdot 365 \mathrm{~d} / \mathrm{yr} \cdot 5.00 \mathrm{E}-8 \mathrm{rem} / \mathrm{pCi} \cdot \frac{10^{12} \mathrm{pCi}}{1 \mathrm{Ci}}$

Dose $=4.57 \mathrm{E}-4$ rem 


$$
\begin{aligned}
\text { Dose } & =\chi \cdot \mathrm{v}_{\mathrm{d}}(\text { soil }) \cdot \frac{1-\exp \left[-\left(\lambda_{\mathrm{i}}+\lambda_{\mathrm{g}}+\lambda_{\mathrm{r}}\right) \cdot \mathrm{T}\right]}{\lambda_{\mathrm{i}}+\lambda_{\mathrm{g}}+\lambda_{\mathrm{r}}} \cdot \mathrm{R} \cdot \mathrm{v}_{\mathrm{d}}(\text { for }) \cdot \mathrm{r} \cdot \mathrm{TF} \cdot \\
& \frac{1-\exp \left[-\lambda_{\mathrm{w}} \cdot \mathrm{t}_{\mathrm{for}}\right]}{\lambda_{\mathrm{w}} \cdot \mathrm{Y}_{\text {for }}} \cdot \mathrm{Q}_{\mathrm{for}} \cdot \mathrm{F}_{\mathrm{b}} \cdot \mathrm{FG} \cdot \mathrm{U}_{\mathrm{b}} \cdot \mathrm{DCF}_{\mathrm{ing}} \\
\text { Dose } & =2.04 \mathrm{E}-2 \mathrm{Ci}-\mathrm{s}^{2} \mathrm{~m}^{3} \cdot 0.001 \mathrm{~m} / \mathrm{s} \cdot \frac{1-\exp \left[-\left(2.30 \mathrm{E}-2 \mathrm{yr}^{-1}+0.0139 \mathrm{yr}^{-1}+5.06 \mathrm{yr}^{-1}\right) \cdot 1 \mathrm{yr}\right]}{2.30 \mathrm{E}-2 \mathrm{yr}^{-1}+0.0139 \mathrm{yr}^{-1}+5.06 \mathrm{yr}^{-1}} \\
& 1.0 \mathrm{E}-5 \mathrm{~m}-1 \cdot 0.01 \mathrm{~m} / \mathrm{s} \cdot 0.2 \cdot 1.0 \cdot \frac{1-\exp \left[-0.0495 \mathrm{~d}^{-1} \cdot 30 \mathrm{~d}\right]}{0.0495 \mathrm{~d}^{-1} \cdot 0.75 \mathrm{~kg} / \mathrm{m}^{2}} \cdot \\
& 50 \mathrm{~kg} / \mathrm{d} \cdot 0.004 \mathrm{~d} / \mathrm{kg} \cdot 0.50 \cdot 0.30 \mathrm{~kg} / \mathrm{d} \cdot 5.0 \mathrm{E}-8 \mathrm{rem} / \mathrm{pCi} \cdot \frac{3.154 \mathrm{E}+7 \mathrm{~s}}{1 \mathrm{yr}} \cdot \frac{10^{12} \mathrm{pCi}}{1 \mathrm{Ci}} \\
\text { Dose } & =7.85 \mathrm{E}-2 \mathrm{rem}
\end{aligned}
$$

6.3.4.4.2.3 Total Long-Term Ingestion Radiation Dose Through the

Meat Pathway-

Dose $=4.57 \mathrm{E}-4 \mathrm{rem}+7.85 \mathrm{E}-2$ rem $=7.90 \mathrm{E}-2$ rem

\subsection{Long-Term Ingestion Radiation Dose Through the Milk}

Pathway-This section provides calculations on root uptake of radioactivity, deposition of resuspended radioactivity, and total long-term ingestion radiation dose. 
Dose $=\chi \cdot \mathrm{v}_{\mathrm{d}}($ soil $) \cdot \frac{1-\exp \left[-\left(\lambda_{\mathrm{i}}+\lambda_{\mathrm{g}}\right) \cdot \mathrm{T}\right]}{\lambda_{\mathrm{i}}+\lambda_{\mathrm{g}}} \cdot \mathrm{B}_{\mathrm{v}} \cdot \frac{1}{\mathrm{P}} \cdot \mathrm{Q}_{\text {for }} \cdot \mathrm{F}_{\mathrm{m}} \cdot \mathrm{FG} \cdot \mathrm{U}_{\mathrm{m}} \cdot \mathrm{DCF}_{\text {ing }}$ Dose $=2.04 \mathrm{E}-2 \mathrm{Ci}-\mathrm{s} / \mathrm{m}^{3} \cdot 0.001 \mathrm{~m} / \mathrm{s} \cdot \frac{1-\exp \left[-\left(2.30 \mathrm{E}-2 \mathrm{yr}^{-1}+0.0139 \mathrm{yr}^{-1}\right) \cdot 1 \mathrm{yr}\right]}{2.30 \mathrm{E}-2 \mathrm{yr}^{-1}+0.0139 \mathrm{yr}^{-1}} \cdot$ $0.010 \frac{\mathrm{pCi} / \mathrm{kg}(\text { wet veg })}{\mathrm{pCi} / \mathrm{kg}(\text { dry soil })} \cdot \frac{1}{240 \mathrm{~kg}(\text { dry soil }) / \mathrm{m}^{2}} \cdot 50 \mathrm{~kg} / \mathrm{d} \cdot 0.012 \mathrm{~d} / \mathrm{L} \cdot 0.50 \cdot$ $0.85 \mathrm{~L} / \mathrm{d} \cdot 365 \mathrm{~d} / \mathrm{yr} \cdot 5.00 \mathrm{E}-8 \mathrm{rem} / \mathrm{pCi} \cdot \frac{10^{12} \mathrm{pCi}}{1 \mathrm{Ci}}$

Dose $=3.88 \mathrm{E}-3 \mathrm{rem}$

\subsection{Deposition of Resuspended Radioactivity-}

Dose $=\chi \cdot \mathrm{v}_{\mathrm{d}}($ soil $) \cdot \frac{1-\exp \left[-\left(\lambda_{\mathrm{i}}+\lambda_{\mathrm{g}}+\lambda_{\mathrm{r}}\right) \cdot \mathrm{T}\right]}{\lambda_{\mathrm{i}}+\lambda_{\mathrm{g}}+\lambda_{\mathrm{r}}} \cdot \mathrm{R} \cdot \mathrm{v}_{\mathrm{d}}($ for $) \cdot \mathrm{r} \cdot \mathrm{TF} \cdot$

$$
\frac{1-\exp \left[-\lambda_{\mathrm{w}} \cdot \mathrm{t}_{\text {for }}\right]}{\lambda_{\mathrm{w}} \cdot \mathrm{Y}_{\text {for }}} \cdot \mathrm{Q}_{\text {for }} \cdot \mathrm{F}_{\mathrm{m}} \cdot \mathrm{FG} \cdot \mathrm{U}_{\mathrm{m}} \cdot \mathrm{DCF}_{\mathrm{ing}}
$$

Dose $=2.04 \mathrm{E}-2 \mathrm{Ci}-\mathrm{s} / \mathrm{m}^{3} \cdot 0.001 \mathrm{~m} / \mathrm{s} \cdot \frac{1-\exp \left[-\left(2.30 \mathrm{E}-2 \mathrm{yr}^{-1}+0.0139 \mathrm{yr}^{-1}+5.06 \mathrm{yr}^{-1}\right) \cdot 1 \mathrm{yr}\right]}{2.30 \mathrm{E}-2 \mathrm{yr}^{-1}+0.0139 \mathrm{yr}^{-1}+5.06 \mathrm{yr}^{-1}} \cdot$

$1.0 \mathrm{E}-5 \mathrm{~m}^{-1} \cdot 0.01 \mathrm{~m} / \mathrm{s} \cdot 0.2 \cdot 1.0 \cdot \frac{1-\exp \left[-0.0495 \mathrm{~d}^{-1} \cdot 30 \mathrm{~d}\right]}{0.0495 \mathrm{~d}^{-1} \cdot 0.75 \mathrm{~kg} / \mathrm{m}^{2}} \cdot$

$50 \mathrm{~kg} / \mathrm{d} \cdot 0.012 \mathrm{~d} / \mathrm{L} \cdot 0.5 \cdot 0.85 \mathrm{~L} / \mathrm{d} \cdot 5.0 \mathrm{E}-8 \mathrm{rem} / \mathrm{pCi} \cdot \frac{3.154 \mathrm{E}+7 \mathrm{~s}}{1 \mathrm{yr}} \cdot \frac{10^{12} \mathrm{pCi}}{1 \mathrm{Ci}}$

Dose $=6.65 \mathrm{E}-1$ rem 
6.3.4.4.3.3 Total Long-Term Ingestion Radiation Dose Through the Milk Pathway -

Dose $=3.88 \mathrm{E}-3 \mathrm{rem}+6.65 \mathrm{E}-1 \mathrm{rem}=6.69 \mathrm{E}-1 \mathrm{rem}$ 


\section{REFERENCES}

ASME (American Society of Mechanical Engineers), 1989, Quality Assurance Requirements for Nuclear Facility Applications, ASME NQA-2-1989, The American Society of Mechanical Engineers, New York, New York.

Baca, R. G. and S.O. Magnuson, 1990, Independent Verification and Benchmark Testing of the UNSAT-H Computer Code, EGG-BEG-8811, EG\&G Idaho, Inc., Idaho Falls, Idaho.

Brumburgh, G. P. and H. P. Alesso, 1993, A Comparison of RISKIND and RADTRAN 4, UCRL-ID-115618, Lawrence Livermore National Laboratory, Livermore, California.

Delorme, 1994, Map 'n Go User's Guide, Delorme Mapping Company, Freeport, Maine.

DOE (U.S. Department of Energy), 1995, Department of Energy Programmatic Spent Nuclear Fuel Management and Idaho National Engineering Laboratory Environmental Restoration and Waste Management Programs Final Environmental Impact Statement, DOE/EIS-0203-F, U.S. Department of Energy, Washington, D.C.

Eggleston, S. L., 1995, Railroad Information Service, P.O. Box 40085, Georgetown, Texas 78628, Letter to Howard K. Pippen, Science Applications International Corporation, San Diego, California.

ICRP (International Commission on Radiological Protection), 1991, 1990 Recommendations of the International Commission on Radiological Protection, ICRP Publication 60, Annals of the ICRP, Volume 21, No. 1-3, Pergamon Press, New York, New York.

Jefferson, R. M., R. E. Luna, J. D. McClure, E. L. Wilmot, 1982, Analysis of Recent Council on Economic Priorities Newsletter, SAND82-1250, Sandia National Laboratories, Albuquerque, New Mexico.

Jefferson, R. M., 1983, Transporting Spent Nuclear Fuel Allegations and Responses, SAND82-2778, Sandia National Laboratories, Albuquerque, New Mexico.

Johnson, P. E., D. S. Joy, D. B. Clarke, J. M. Jacobi, 1993a, HIGHWAY 3.1 - An Enhanced Highway Routing Model: Program Description, Methodology, and Revised User 's Manual, ORNL/TM-12124, Oak Ridge National Laboratory, Oak Ridge, Tennessee.

Johnson, P. E., D. S. Joy, D. B. Clarke, J. M. Jacobi, 1993b, INTERLINE 5.0 - An Expanded Railroad Routing Model: Program Description, Methodology, and Revised User's Manual, ORNL/TM-12090, Oak Ridge National Laboratory, Oak Ridge, Tennessee.

Lauridsen, B. and P. Hedemann-Jensen, 1983, "Shielding Factors for Vehicles to $\gamma$ Radiation from Activity Deposited on Structures and Ground Surfaces," Health Physics, 45, 6, 1983, pp. 1039-1045.

Maheras, S. J., P. D. Ritter, P. R. Leonard, R. E. Moore, 1994, "Benchmarking of the CAP-88 and GENII Computer Codes Using 1990 and 1991 Monitored Atmospheric Releases from the Idaho National Engineering Laboratory," Health Physics, 67, 5, pp. 509-517. 
Neuhauser, K. S. and F. L. Kanipe, 1995, RADTRAN 4, Volume II: Technical Manual, SAND89-2370, Revision 1, Sandia National Laboratories, Albuquerque, New Mexico.

NRC (U.S. Nuclear Regulatory Commission), 1975, Reactor Safety Study, WASH-1400, NUREG-75/014, U.S. Nuclear Regulatory Commission, Washington, D.C.

NRC (U.S. Nuclear Regulatory Commission), 1977a, Final Environmental Impact Statement on the Transportation of Radioactive Material by Air and Other Modes, NUREG-0170, U.S. Nuclear Regulatory Commission, Washington, D.C.

NRC (U.S. Nuclear Regulatory Commission), 1977b, Regulatory Guide 1.109: Calculation of Annual Doses to Man from Routine Releases of Reactor Effluents for the Purpose of Evaluating Compliance with 10 CFR Part 50, Appendix I, Revision 1, U.S. Nuclear Regulatory Commission, Washington, D.C.

Resnikoff, M., L. Birnbaum, L. Audin, 1982, The Latest Nuclear Dilemma: Waste Shipment Peril Explored, CEP Publication N 82-1, Council on Economic Priorities, New York, New York.

Resnikoff, M., 1983, The Next Nuclear Gamble: Transportation and Storage of Nuclear Waste, Council on Economic Priorities, New York, New York.

Resnikoff, M., 1990, Probabilistic Risk Assessment and Nuclear Waste Transportation: A Case Study of the Use of RADTRAN in the 1986 Environmental Assessment for Yucca Mountain, NWPO-TN-006-90, Nuclear Waste Project Office, Las Vegas, Nevada.

Weiner, R. F. and K. S. Neuhauser, 1992, "Conservatism of RADTRAN Line-Source Model for Estimating Worker Exposures," PATRAM '92: 10th International Symposium on the Packaging and Transportation of Radioactive Materials, National Technical Information Service, Springfield, Virginia, CONF-920905, pp. 211-217.

Wooden, D. G., 1986, Railroad Transportation of Spent Nuclear Fuel, SAND86-7083, Sandia National Laboratories, Albuquerque, New Mexico.

Yuan, Y. C., S. Y. Chen, D. J. LePoire, R. Rothman, 1993, RISKIND - A Computer Program for Calculating Radiological Consequences and Health Risks from Transportation of Spent Nuclear Fuel, ANL/EAIS-6, Rev. 0, Argonne National Laboratory, Argonne, Illinois. 


\section{APPENDIX A}

\section{RADTRAN 4 OUTPUT FOR INCIDENT-FREE UNIT RISK FACTORS}


A-2 
A.1 TURFR.OUT

UNIT RISK FACTORS FOR TRUCKS IN RURAL POPULATION ZONE 


$$
\text { A-4 }
$$




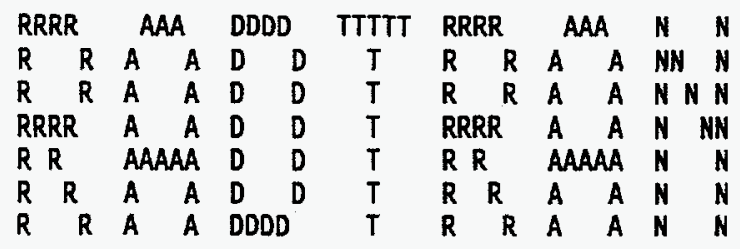

RADTRAN 4.0.17 VERSION DATE: NOVEMBER 8, 1994

MODE DESCRIPTIONS

$\begin{array}{cl}\text { NUMBER } & \text { NAME } \\ 1 & \text { TRUCK } \\ 2 & \text { RAIL } \\ 3 & \text { BARGE } \\ 4 & \text { SHIP } \\ 5 & \text { CARGO AIR } \\ 6 & \text { PASS AIR } \\ 7 & \text { P-VAN } \\ 8 & \text { CVAN-T } \\ 9 & \text { CVAN-R } \\ 10 & \text { CVAN-CA }\end{array}$

CHARACTERIZATION

LONG HAUL VEHICLE

COMMERCIAL TRAIN

INLAND VESSEL

OPEN SEA VESSEL

CARGO AIRCRAFT

PASSENGER AIRCRAFT

PASSENGER VAN

COMMERCIAL VAN

COMMERCIAL VAN

COMMERCIAL VAN 
ECHO CHECK

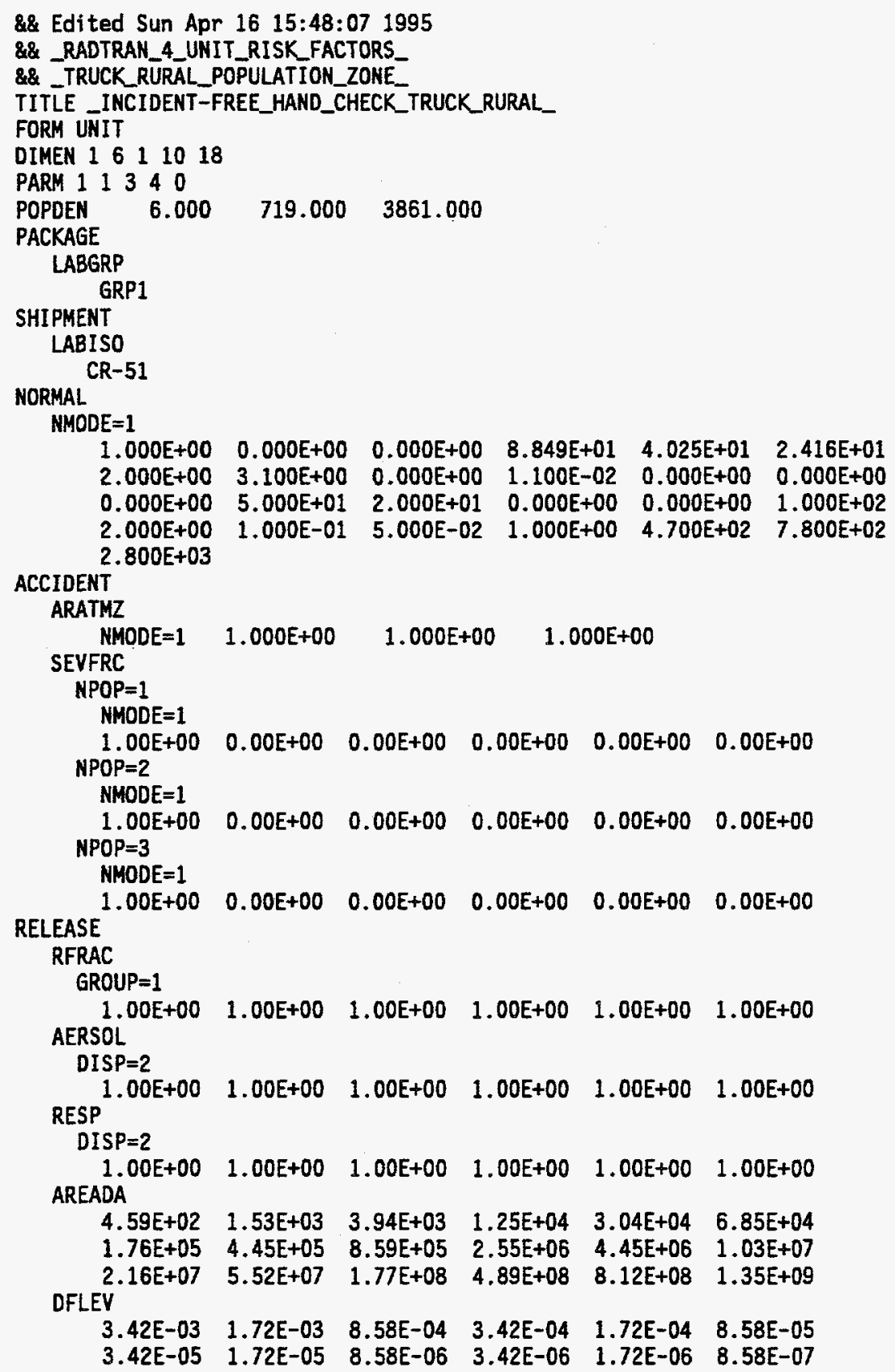

$\begin{array}{llllll}3.42 E-03 & 1.72 E-03 & 8.58 E-04 & 3.42 E-04 & 1.72 E-04 & 8.58 E-05\end{array}$

$3.42 E-05 \quad 1.72 E-05 \quad 8.58 E-06 \quad 3.42 E-06 \quad 1.72 E-06 \quad 8.58 E-07$ 


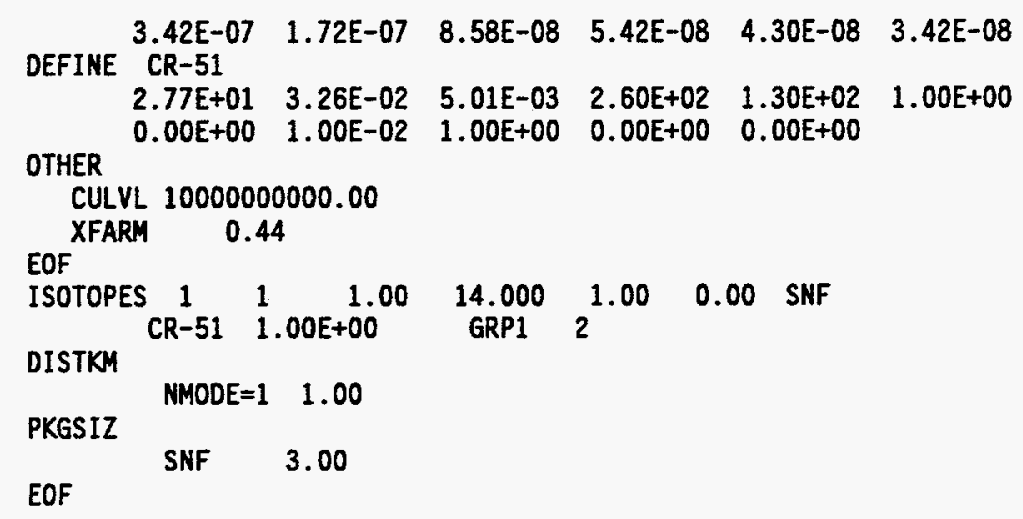


RUN DATE: [ 16-APR-95 AT 15:48:49]

_INCIDENT-FREE_HAND_CHECK_TRUCK_RURAL_

\begin{tabular}{lc}
\multicolumn{1}{c}{ ZONE } & POPULATION DENSITY \\
RURAL & (PERSONS PER SQ KM) \\
SURURBAN & 6. \\
URBAN & 719. \\
& 3861.
\end{tabular}

PACKAGE CHARACTERISTICS

$\begin{array}{cccc}\text { FOR } & \text { DIMENSION } & \text { EFFECTIVE } & \text { K(0) } \\ \text { MATERIAL } & \text { (METERS) } & \text { DIMENSION } & \text { METERS SQ. } \\ \text { SNF } & 3.000 E+00 & 3.000 E+00 & 6.250 E+00\end{array}$

$K(0)$ IS TI TO DOSE RATE CONVERSION FACTOR

PACKAGE HANDLING THRESHOLDS (METERS)

PKGSZ1 $=5.000 E-01$

PKGSZ2 $=1.000 E+00$

PACKAGES .LE. PKGSZI ARE HAND CARRIED

PACKAGES .GT. PKGSZ1 AND .LE. PKGSZ2 ARE hANDLED BY SMALL EQUIPMENT

PACKAGES .GT. PKGSZZ ARE HANDLED BY HEAVY EQUIPMENT

MATERIAL CHARACTERISTICS

$\begin{array}{rrr}\text { FRACTION } & \text { FRACTION } \\ \text { MATERIAL } & \text { OF GAMMA } & \text { OF NEUTRON } \\ \text { SNF } & 1.000 E+00 & 0.000 E+00\end{array}$


RUN DATE: [ 16-APR-95 AT 15:48:49]

_INCIDENT-FREE_HANO_CHECK_TRUCK_RURAL_

MODE CHARACTERISTICS

$\begin{array}{ccccccc}\text { MODE } & \begin{array}{c}\text { DISTANCE } \\ \text { TRAVELED }\end{array} & \begin{array}{c}\text { EXCLUSIVE } \\ \text { USE }\end{array} & \begin{array}{c}\text { NUMBER OF } \\ \text { SHIPMENTS }\end{array} & \text { MATERIALS } & \begin{array}{c}\text { TRANSPORT } \\ \text { INDEX (TI) }\end{array} & \begin{array}{c}\text { PACKAGES/ } \\ \text { SHIPMENT }\end{array} \\ \text { TRUCK } & 1.00 E+00 & \text { NO } & 1.00 E+00 & & & \\ & & & & \text { SNF } & 1.40 E+01 & 1.00 E+00\end{array}$

BUILDING SHIELDING OPTION $=3$

(1=TOTAL SHIELDING, 2=PARTIAL SHIELDING, 3=NO SHIELDING)

$R P D=6.000 E+00$

(RATIO OF PEDESTRIAN DENSITY (PEDESTRIAN/KM SQ OF SIDEWALK)

TO POPULATION DENSITY (PEOPLE/KM SQ IN URBAN AREAS)

$R R=1.000 E+00$

(TRANSMISSION FACTOR FOR RURAL AREAS)

RS $=1.000 E+00$

(TRANSMISSION FACTOR FOR SUBURBAN AREAS)

$\mathrm{RU}=1.000 \mathrm{E}+00$

(TRANSHISSION FACTOR FOR URBAN AREAS) 


\begin{tabular}{|c|c|c|}
\hline & DNORML & INPUT \\
\hline 1 & FRACTION OF TRAVEL & $1.000 E+00$ \\
\hline 2 & $\begin{array}{l}\text { IN RURAL POPULATION ZONE } \\
\text { FRACTION OF TRAVEL } \\
\text { IN SUBURBAN POPULATION ZONE }\end{array}$ & $0.000 E+00$ \\
\hline 3 & $\begin{array}{l}\text { FRACTION OF TRAVEL } \\
\text { IN URBAN POPULATION ZONE }\end{array}$ & $0.000 E+00$ \\
\hline 4 & $\begin{array}{l}\text { VELOCITY IN RURAL POPULATION } \\
\text { ZONE (KILOMETERS/HOUR) }\end{array}$ & $8.849 E+01$ \\
\hline 5 & $\begin{array}{l}\text { VELOCITY IN SUBURBAN POP. ZONE } \\
\text { (KILOMETERS/HOUR) }\end{array}$ & $5 E+01$ \\
\hline 6 & $\begin{array}{l}\text { VELOCITY IN URBAN POPULATION } \\
\text { ZONE (KILONETERS/HOUR) }\end{array}$ & $2.416 E+01$ \\
\hline 7 & MUMBER OF CREMMEN & $2.000 E+00$ \\
\hline 8 & $\begin{array}{l}\text { DISTANCE FROM SOURCE TO CREW } \\
\text { (METERS) }\end{array}$ & $3.100 E+00$ \\
\hline 9 & NUMBER OF HANDLINGS & $0.000 E+00$ \\
\hline & STOP TIME PER KM (HR/KM) & $1.100 E-02$ \\
\hline 11 & $\begin{array}{l}\text { MINIMUM STOP TIME PER TRIP } \\
\text { (HR) }\end{array}$ & $E+\infty$ \\
\hline & ZERO STOP TIME PER TRIP (HR) & $0.000 E+00$ \\
\hline & $\begin{array}{l}\text { MINIMUM NUMBER OF RAIL CLASSIF } \\
\text { ICATIONS/INSPECTIONS }\end{array}$ & \\
\hline & PERSONS EXPOSED WHILE STOPPED & $5.000 E+01$ \\
\hline & $\begin{array}{l}\text { AVERAGE EXPOSURE DISTANCE } \\
\text { WHILE STOPPED (METERS) }\end{array}$ & $2.000 E+01$ \\
\hline 6 & $\begin{array}{l}\text { STORAGE TIME PER SHIPMENT } \\
\text { (HR) }\end{array}$ & $0.000 E+00$ \\
\hline 7 & $\begin{array}{l}\text { NUMBER OF EXPOSED PERSONS } \\
\text { DURING STORAGE }\end{array}$ & $0.000 E+00$ \\
\hline 8 & $\begin{array}{l}\text { AVERAGE EXPOSURE DISTANCE } \\
\text { WHILE IN STORAGE (METERS) }\end{array}$ & $1.000 E+02$ \\
\hline & $\begin{array}{l}\text { NUMBER OF PEOPLE PER VEHICLE } \\
\text { ON LINK }\end{array}$ & $2.000 E+00$ \\
\hline & $\begin{array}{l}\text { FRACTION OF URBAN TRAVEL } \\
\text { DURING RUSH HOUR TRAFFIC }\end{array}$ & $1.000 E-01$ \\
\hline & $\begin{array}{l}\text { FRACTION OF URBAN TRAVEL } \\
\text { ON CITY STREETS }\end{array}$ & $5.000 E-02$ \\
\hline & $\begin{array}{l}\text { FRACTION OF RURAL-SUBURBAN } \\
\text { TRAVEL ON FREEWAYS }\end{array}$ & $1.000 E+00$ \\
\hline & $\begin{array}{l}\text { *TRAFFIC COUNT PASSING A } \\
\text { SPECIFIC POINT-RURAL ZONE }\end{array}$ & $4.700 E+02$ \\
\hline & 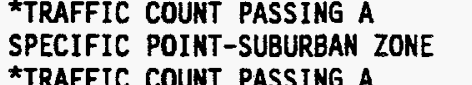 & $7.800 E+02$ \\
\hline & POINT-URBAN ZONE & $2.800 E+03$ \\
\hline
\end{tabular}


RUN DATE: [ 16-APR-95 AT 15:48:49]

_INCIDENT-FREE_HAND_CHECK_TRUCK_RURAL_

ISOTOPE RELATED DATA

\begin{tabular}{|c|c|c|c|c|c|c|c|}
\hline NUCLIDE & $\begin{array}{l}\text { CURIES } \\
\text { PER PKG }\end{array}$ & $\begin{array}{l}\text { RELEAS } \\
\text { GROUF }\end{array}$ & $\begin{array}{l}\text { RESL } \\
\text { FAC }\end{array}$ & $\begin{array}{l}\text { JSP } \\
\text { TOR }\end{array}$ & $\begin{array}{l}\text { LUNG } \\
\text { TYPE }\end{array}$ & $\begin{array}{l}\text { OISPERS. } \\
\text { CATEGORY }\end{array}$ & $\begin{array}{cc}\text { 1YR INHAL REM/CI } \\
\text { LUNG } & \text { MARROW }\end{array}$ \\
\hline $\begin{array}{r}\text { SNF } \\
\text { CR-51 }\end{array}$ & $1.00 E+00$ & GRF & $1.32 E$ & $\pm+\infty$ & 1 & 2 & $0.00 \mathrm{E}+00 \quad 0.00 \mathrm{E}+00$ \\
\hline NUCLIDE & $\begin{array}{l}\text { HALF } \\
\text { LIFE }\end{array}$ & $\begin{array}{l}\text { GAMMA } \\
\text { ENERGY }\end{array}$ & $\begin{array}{l}\text { CLOUD } \\
\text { FACTOR }\end{array}$ & & $\begin{array}{l}\text { TRAI } \\
\text { CROPS }\end{array}$ & $\begin{array}{l}\text { SFER } \\
\text { SOIL }\end{array}$ & $\begin{array}{l}\text { DEPOS } \\
\text { SPEED }\end{array}$ \\
\hline $\begin{array}{r}\text { SNF } \\
C R-51\end{array}$ & 2.77E+01 & $3.26 \mathrm{E}-02$ & $5.01 E-03$ & & $00 E+00$ & $0.00 E+00$ & $1.00 \mathrm{E}-02$ \\
\hline NUCLIDE & \multicolumn{7}{|c|}{$\begin{array}{l}\text { 50-YR EFFECTIVE REM/CI } \\
\text { INHALE INGEST }\end{array}$} \\
\hline $\begin{array}{r}\text { SNF } \\
\text { CR-51 }\end{array}$ & $2.60 \mathrm{E}$ & $1.30 \mathrm{E}$ & & & & & \\
\hline
\end{tabular}


_INCIDENT-FREE_HAND_CHECK_TRUCK_RURAL_

RELEASE RELATED DATA

ACCIDENT RATES (PER KM)

MODE RURAL SUBURBAN URBAN

TRUCK $1.000 E+00 \quad 1.000 E+00 \quad 1.000 E+00$

RELEASE FRACTIONS

GROUP SEVER: 1 SEVER: 2 SEVER: 3 SEVER: 4 SEVER: 5 SEVER: 6

$1 \quad 1.00 E+00 \quad 1.00 E+00 \quad 1.00 E+00 \quad 1.00 E+00 \quad 1.00 E+00 \quad 1.00 E+00$

\section{ACCIDENT SEVERITY FRACTIONS}

FOR TRUCK

ZONE SEVER: 1 SEVER: 2 SEVER: 3 SEVER: 4 SEVER: 5 SEVER: 6

$1 \quad 1.00 E+00 \quad 0.00 E+00 \quad 0.00 E+00 \quad 0.00 E+00 \quad 0.00 E+00 \quad 0.00 E+00$

$2 \quad 1.00 E+00 \quad 0.00 E+00 \quad 0.00 E+00 \quad 0.00 E+00 \quad 0.00 E+00 \quad 0.00 E+00$

$\begin{array}{lllllll}3 & 1.00 E+00 & 0.00 E+00 & 0.00 E+00 & 0.00 E+00 & 0.00 E+00 & 0.00 E+00\end{array}$ 
RUN DATE: [ 16-APR-95 AT 15:48:49 ]

_INCIDENT-FREE_HAND_CHECK_TRUCK_RURAL_

AEROSOLIZED FRACTION OF RELEASED MATERIAL

$\begin{array}{cllllll}\text { DISP CAT } & \text { SEVER: } 1 & \text { SEVER: } 2 & \text { SEVER: } 3 & \text { SEVER: } 4 & \text { SEVER: } 5 & \text { SEVER: } 6 \\ 1 & 0.00 E+00 & 0.00 E+00 & 0.00 E+00 & 0.00 E+00 & 0.00 E+00 & 0.00 E+00 \\ 2 & 1.00 E+00 & 1.00 E+00 & 1.00 E+00 & 1.00 E+00 & 1.00 E+00 & 1.00 E+00 \\ 3 & 1.00 E-02 & 1.00 E-02 & 1.00 E-02 & 1.00 E-02 & 1.00 E-02 & 1.00 E-02 \\ 4 & 5.00 E-02 & 5.00 E-02 & 5.00 E-02 & 5.00 E-02 & 5.00 E-02 & 5.00 E-02 \\ 5 & 1.00 E-01 & 1.00 E-01 & 1.00 E-01 & 1.00 E-01 & 1.00 E-01 & 1.00 E-01 \\ 6 & 1.00 E+00 & 1.00 E+00 & 1.00 E+00 & 1.00 E+00 & 1.00 E+00 & 1.00 E+00 \\ 7 & 1.00 E+00 & 1.00 E+00 & 1.00 E+00 & 1.00 E+00 & 1.00 E+00 & 1.00 E+00 \\ 8 & 1.00 E+00 & 1.00 E+00 & 1.00 E+00 & 1.00 E+00 & 1.00 E+00 & 1.00 E+00 \\ 9 & 1.00 E+00 & 1.00 E+00 & 1.00 E+00 & 1.00 E+00 & 1.00 E+00 & 1.00 E+00 \\ 10 & 1.00 E+00 & 1.00 E+00 & 1.00 E+00 & 1.00 E+00 & 1.00 E+00 & 1.00 E+00 \\ 11 & 1.00 E+00 & 1.00 E+00 & 1.00 E+00 & 1.00 E+00 & 1.00 E+00 & 1.00 E+00\end{array}$

FRACTION OF AEROSOLS BELOW 10 MICRONS AED

$\begin{array}{cllllll}\text { DISP CAT } & \text { SEVER: } 1 & \text { SEVER: } 2 & \text { SEVER: } 3 & \text { SEVER: } 4 & \text { SEVER: } 5 & \text { SEVER: } 6 \\ 1 & 0.00 E+00 & 0.00 E+00 & 0.00 E+00 & 0.00 E+00 & 0.00 E+00 & 0.00 E+00 \\ 2 & 1.00 E+00 & 1.00 E+00 & 1.00 E+00 & 1.00 E+00 & 1.00 E+00 & 1.00 E+00 \\ 3 & 5.00 E-02 & 5.00 E-02 & 5.00 E-02 & 5.00 E-02 & 5.00 E-02 & 5.00 E-02 \\ 4 & 5.00 E-02 & 5.00 E-02 & 5.00 E-02 & 5.00 E-02 & 5.00 E-02 & 5.00 E-02 \\ 5 & 5.00 E-02 & 5.00 E-02 & 5.00 E-02 & 5.00 E-02 & 5.00 E-02 & 5.00 E-02 \\ 6 & 5.00 E-02 & 5.00 E-02 & 5.00 E-02 & 5.00 E-02 & 5.00 E-02 & 5.00 E-02 \\ 7 & 1.00 E+00 & 1.00 E+00 & 1.00 E+00 & 1.00 E+00 & 1.00 E+00 & 1.00 E+00 \\ 8 & 1.00 E+00 & 1.00 E+00 & 1.00 E+00 & 1.00 E+00 & 1.00 E+00 & 1.00 E+00 \\ 9 & 1.00 E+00 & 1.00 E+00 & 1.00 E+00 & 1.00 E+00 & 1.00 E+00 & 1.00 E+00 \\ 10 & 1.00 E+00 & 1.00 E+00 & 1.00 E+00 & 1.00 E+00 & 1.00 E+00 & 1.00 E+00 \\ 11 & 1.00 E+00 & 1.00 E+00 & 1.00 E+00 & 1.00 E+00 & 1.00 E+00 & 1.00 E+00\end{array}$


RUN DATE: [ 16-APR-95 AT 15:48:49]

_INCIDENT-FREE_HAND_CHECK_TRUCK_RURAL_

COST RELATED DATA

EMERGENCY RESPONSE COST

SEVER: 1 SEVER: 2 SEVER: 3 SEVER: 4 SEVER: 5 SEVER: 6

$0.00 E+00 \quad 0.00 E+00 \quad 0.00 E+00 \quad 0.00 E+00 \quad 0.00 E+00 \quad 0.00 E+00$

ON-SCENE COSTS

(RF=RELEASE FRACTION)

$R F=0$. $\quad 0 .<R F<=.01 \quad .01<R F<=0.1 \quad .1<R F<=1$.

0 .

0

0 .

0. 
RUN DATE: [ 16-APR-95 AT 15:48:49]

_INCIDENT-FREE_HAND_CHECK_TRUCK_RURAL_

HEALTH RELATED DATA

\section{EARLY FATALITY PROBABILITIES}

$\begin{array}{rrrrr}\text { DOSE (REM) } & \text { LUNG-1 } & \text { LUNG-2 } & \text { LUNG-3 } & \text { MARROW } \\ 100000.000 & 1.000 E+00 & 1.000 E+00 & 1.000 E+00 & 1.000 E+00 \\ 80000.000 & 1.000 E+00 & 8.500 E-01 & 8.000 E-01 & 1.000 E+00 \\ 70000.000 & 1.000 E+00 & 8.000 E-01 & 5.000 E-01 & 1.000 E+00 \\ 40000.000 & 1.000 E+00 & 7.000 E-01 & 0.000 E+00 & 1.000 E+00 \\ 30000.000 & 1.000 E+00 & 5.000 E-01 & 0.000 E+00 & 1.000 E+00 \\ 25000.000 & 1.000 E+00 & 2.000 E-01 & 0.000 E+00 & 1.000 E+00 \\ 20000.000 & 1.000 E+00 & 8.000 E-02 & 0.000 E+00 & 1.000 E+00 \\ 10000.000 & 6.000 E-01 & 0.000 E+00 & 0.000 E+00 & 1.000 E+00 \\ 8000.000 & 1.000 E-01 & 0.000 E+00 & 0.000 E+00 & 1.000 E+00 \\ 6000.000 & 6.000 E-02 & 0.000 E+00 & 0.000 E+00 & 1.000 E+00 \\ 4000.000 & 3.000 E-02 & 0.000 E+00 & 0.000 E+00 & 1.000 E+00 \\ 3000.000 & 0.000 E+00 & 0.000 E+00 & 0.000 E+00 & 1.000 E+00 \\ 2000.000 & 0.000 E+00 & 0.000 E+00 & 0.000 E+00 & 1.000 E+00 \\ 1000.000 & 0.000 E+00 & 0.000 E+00 & 0.000 E+00 & 1.000 E+00 \\ 800.000 & 0.000 E+00 & 0.000 E+00 & 0.000 E+00 & 9.960 E-01 \\ 700.000 & 0.000 E+00 & 0.000 E+00 & 0.000 E+00 & 9.000 E-01 \\ 600.000 & 0.000 E+00 & 0.000 E+00 & 0.000 E+00 & 4.000 E-01 \\ 500.000 & 0.000 E+00 & 0.000 E+00 & 0.000 E+00 & 5.000 E-02 \\ 400.000 & 0.000 E+00 & 0.000 E+00 & 0.000 E+00 & 0.000 E+00 \\ 300.000 & 0.000 E+00 & 0.000 E+00 & 0.000 E+00 & 0.000 E+00 \\ 100.000 & 0.000 E+00 & 0.000 E+00 & 0.000 E+00 & 0.000 E+00 \\ 75.000 & 0.000 E+00 & 0.000 E+00 & 0.000 E+00 & 0.000 E+00 \\ 50.000 & 0.000 E+00 & 0.000 E+00 & 0.000 E+00 & 0.000 E+00 \\ 30.000 & 0.000 E+00 & 0.000 E+00 & 0.000 E+00 & 0.000 E+00 \\ 15.000 & 0.000 E+00 & 0.000 E+00 & 0.000 E+00 & 0.000 E+00 \\ 5.000 & 0.000 E+00 & 0.000 E+00 & 0.000 E+00 & 0.000 E+00 \\ 1.000 & 0.000 E+00 & 0.000 E+00 & 0.000 E+00 & 0.000 E+00 \\ 0.100 & 0.000 E+00 & 0.000 E+00 & 0.000 E+00 & 0.000 E+00 \\ 0.010 & 0.000 E+00 & 0.000 E+00 & 0.000 E+00 & 0.000 E+00 \\ 0.010 & 0.000 E+00 & 0.000 E+00 & 0.000 E+00 & 0.000 E+00\end{array}$


_INCIDENT-FREE_HAND_CHECK_TRUCK_RURAL_

DISPERSAL ACCIDENT INPUT

$\begin{array}{cc}\begin{array}{c}\text { AREADA } \\ \text { (M SQ) }\end{array} & \begin{array}{c}\text { DILUTION } \\ \text { FACTOR* }\end{array} \\ 4.590 E+02 & 3.420 E-03 \\ 1.530 E+03 & 1.720 E-03 \\ 3.940 E+03 & 8.580 E-04 \\ 1.250 E+04 & 3.420 E-04 \\ 3.040 E+04 & 1.720 E-04 \\ 6.850 E+04 & 8.580 E-05 \\ 1.760 E+05 & 3.420 E-05 \\ 4.450 E+05 & 1.720 E-05 \\ 8.590 E+05 & 8.580 E-06 \\ 2.550 E+06 & 3.420 E-06 \\ 4.450 E+06 & 1.720 E-06 \\ 1.030 E+07 & 8.580 E-07 \\ 2.160 E+07 & 3.420 E-07 \\ 5.520 E+07 & 1.720 E-07 \\ 1.770 E+08 & 8.580 E-08 \\ 4.890 E+08 & 5.420 E-08 \\ 8.120 E+08 & 4.300 E-08 \\ 1.350 E+09 & 3.420 E-08\end{array}$

- DILUTION FACTOR UNITS ARE (CI-SEC/M**3/CI-RELEASED)

NON-DISPERSAL ACCIDENT INPUT

$\begin{array}{lll} & \text { RADIST(M) } & \\ \text { RURAL } & \text { SUBURBAN } & \text { URBAN } \\ 3.050 E+00 & 3.050 E+00 & 3.050 E+00 \\ 6.100 E+00 & 6.100 E+00 & 6.100 E+00 \\ 9.100 E+00 & 9.100 E+00 & 9.100 E+00 \\ 1.220 E+01 & 1.220 E+01 & 1.220 E+01 \\ 1.520 E+01 & 1.520 E+01 & 1.520 E+01 \\ 3.050 E+01 & 3.050 E+01 & 3.050 E+01 \\ 6.100 E+01 & 6.100 E+01 & 6.100 E+01 \\ 9.140 E+01 & 9.140 E+01 & 9.140 E+01 \\ 1.524 E+02 & 1.524 E+02 & 1.524 E+02 \\ 3.050 E+02 & 3.050 E+02 & 3.050 E+02\end{array}$

BUILDING DOSE FACTOR

FRACTION OF LAND UNDER CULTIVATION

CONTAMINATION CLEAN UP LEVEL (UCI/M**2)

BREATHING RATE ( $\left.H^{\star \star} 3 / \mathrm{SEC}\right)$
$=8.600 \mathrm{E}-03$

$=4.400 \mathrm{E}-01$

$=1.000 \mathrm{E}+09$

$=3.300 \mathrm{E}-04$ 
_INCIDENT-FREE_HAND_CHECK_TRUCK_RURAL_

REGULATORY CHECKS

MODE 1 HAS BEEN REDESIGNATED AS EXCLUSIVE USE

FOR THE SHIPMENT OF SNF BY MODE 1

THE DOSE RATE AT 2 METERS COULD EXCEED 10 MR/HR

PPS*TI HAS BEEN RESET TO EQUAL 14.00

FOR THE SHIPMENT OF

SNF BY MODE 1

THE DOSE RATE IN THE CREW COMPARTMENT COULD EXCEED 2 MREM/HR

THE DOSE RATE HAS BEEN RESET FROM 9.11 TO 2 FOR CREW CALCULATIONS 
RUN DATE: [ 16-APR-95 AT 15:48:49]

_INCIDENT-FREE_HAND_CHECK_TRUCK_RURAL_

\section{INCIDENT-FREE SUMMARY}

INCIDENT-FREE POPULATION EXPOSURE IN PERSON-REM

PASSENGR CREW HANDLERS OFF LINK ON LINK STOPS STORAGE TOTALS LINK $10.00 E+00 \quad 4.56 E-05 \quad 0.00 E+00 \quad 1.23 E-07 \quad 5.03 E-06 \quad 1.20 E-04 \quad 0.00 E+00 \quad 1.71 E-04$

TOTALS: $0.00 E+00 \quad 4.56 E-05 \quad 0.00 E+00 \quad 1.23 E-07 \quad 5.03 E-06 \quad 1.20 E-04 \quad 0.00 E+00 \quad 1.71 E-04$

MAXIMUM INDIVIDUAL IN-TRANSIT DOSE

LINK 1 3.34E-07 REM 
_INCIDENT-FREE_HAND_CHECK_TRUCK_RURAL_

INCIDENT-FREE IMPORTANCE ANALYSIS SUMMARY FOR LINK 1

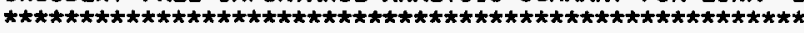

INDEX DESCRIPTION OF PARAMETER

1 DISTANCE TRAVELED

2 NUMBER OF SHIPMENTS

PACKAGES PER SHIPMENT

$K$ ZERO

DOSE RATE (TRANSPORT INDEX)

PERSONS EXPOSED WHILE STOPPED

STOP TIME

FRACTION OF TRAVEL - RURAL

NUMBER OF CREW MEMBERS

FRACTION OF TRAVEL ON FREEWAYS

NUMBER OF PEOPLE PER VEHICLE

TRAFFIC COUNT - RURAL

POPULATION DENSITY - RURAL

NUMBER OF HANDLINGS

DISTANCE FROM SOURCE TO CREW

EXPOSURE TIME FOR HANDLERS

PERSONS EXPOSED PER HANDLING

NUMBER OF FLIGHT ATTENDANTS

TRAFFIC COUNT - URBAN

TRAFFIC COUNT - SUBURBAN

FRACTION OF RUSH HOUR TRAVEL

HANDLER EXPOSURE DISTANCE

STORAGE EXPOSURE DISTANCE

NUMBER OF PERSONS EXPOSED DURING STORAGE

SUBURBAN SHIELDING FACTOR (RS)

VELOCITY - SUBURBAN

POPULATION DENSITY - SUBURBAH

FRACTION OF TRAVEL - SUBURBAN

RATIO OF PEDESTRIAN DENSITY (RPD)

FRACTION OF TRAVEL - URBAN

POPULATION DENSITY - URBAN

STORAGE TIME PER SHIPMENT

URBAN SHIELDING FACTOR (RU)

FRACTION OF TRAVEL ON CITY STREETS

VELOCITY - URBAN

RURAL SHIELDING FACTOR (RR)

VELOCITY - RURAL

EXPOSURE DISTANCE WHILE STOPPED
IMPORTANCE

$1.710 \mathrm{E}-06$

$1.710 \mathrm{E}-06$

$1.255 E-06$

$1.255 E-06$

$1.255 \mathrm{E}-06$

1.203E-06

$1.203 E-06$

$5.072 \mathrm{E}-07$

4.556E-07

$5.152 E-08$

$5.029 \mathrm{E}-08$

$5.029 \mathrm{E}-08$

$1.234 \mathrm{E}-09$

$0.000 E+00$

$0.000 E+00$

$0.000 \mathrm{E}+00$

$0.000 E+00$

$0.000 E+00$

$0.000 E+00$

$0.000 E+00$

$0.000 E+00$

$0.000 E+00$

$0.000 E+00$

$0.000 \mathrm{E}+00$

$0.000 E+00$

$0.000 E+00$

$0.000 E+00$

$0.000 E+00$

$0.000 E+00$

$0.000 E+00$

$0.000 E+00$

$0.000 E+00$

$0.000 E+00$

$0.000 E+00$

$0.000 E+00$

$0.000 E+00$

$-5.575 E-07$

$-2.406 E-06$

THE IMPORTANCE VALUE ESTIMATES THE PERSON-REM INFLUENCE OF A ONE PERCENT INCREASE IN THE PARAMETER

TOTAL EXPOSED POPULATION: INCIDENT-FREE
TRUCK RURAL
9.60E+00 PERSONS
TRUCK SUBURBAN
$0.00 E+00$ PERSONS
TRUCK URBAN
$0.00 E+00$ PERSONS
TOTAL 9.60E+00 PERSONS

TOTAL EXPOSED POPULATION: ACCIDENT

(PERSONS UNDER PLUME FOOTPRINT FOR A SINGLE ACCIDENT)
TRUCK RURAL
$0.00 E+00$ PERSONS
TRUCK SUBURBAN
$0.00 E+00$ PERSONS
TRUCK URBAN
$0.00 E+00$ PERSONS

EOI

END OF RUN 
A-20 


\section{A.2 TURFS.OUT \\ UNIT RISK FACTORS FOR TRUCKS IN SUBURBAN POPULATION ZONE}


A-22 


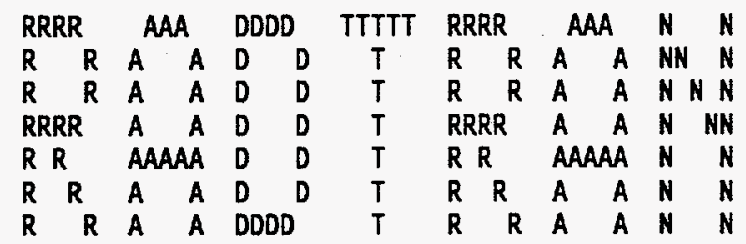

RADTRAN 4.0.17 VERSION DATE: NOVEMBER 8, 1994

MODE DESCRIPTIONS

$\begin{array}{cll}\text { NUMBER } & \text { NAME } & \text { CHARACTERIZATION } \\ 1 & \text { TRUCK } & \text { LONG HAUL VEHICLE } \\ 2 & \text { RAIL } & \text { COMMERCIAL TRAIN } \\ 3 & \text { BARGE } & \text { INLAND VESSEL } \\ 4 & \text { SHIP } & \text { OPEN SEA VESSEL } \\ 5 & \text { CARGO AIR } & \text { CARGO AIRCRAFT } \\ 6 & \text { PASS AIR } & \text { PASSENGER AIRCRAFT } \\ 7 & \text { P-VAN } & \text { PASSENGER VAN } \\ 8 & \text { CVAN-T } & \text { COMMERCIAL VAN } \\ 9 & \text { CVAN-R } & \text { COMMERCIAL VAN } \\ 10 & \text { CVAN-CA } & \text { COMMERCIAL VAN }\end{array}$




\section{ECHO CHECK}

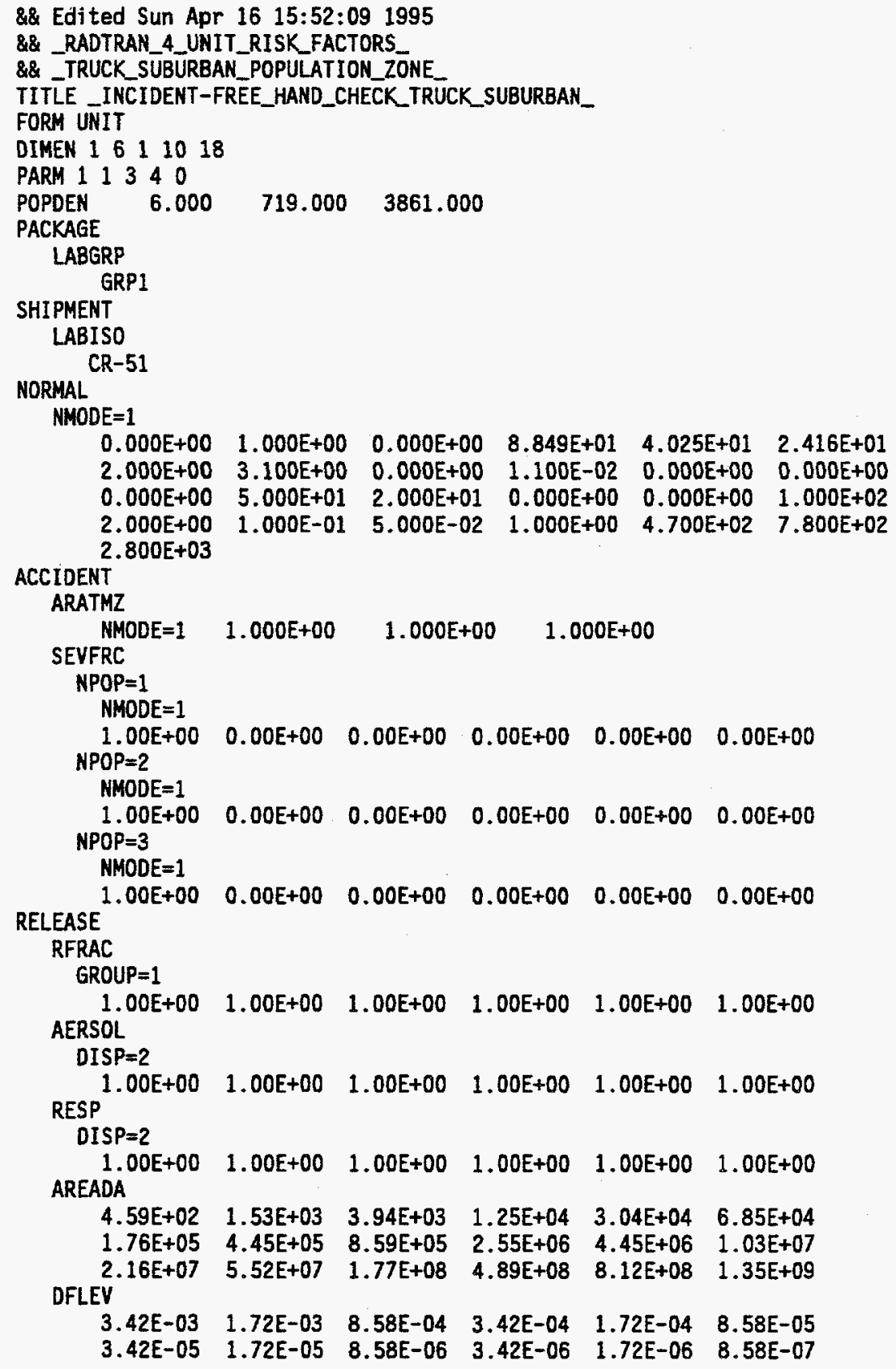


_INCIDENT-FREE_HAND_CHECK_TRUCK_SUBURBAN_

3.42E-07 $\quad 1.72 E-07 \quad 8.58 E-08 \quad 5.42 E-08 \quad 4.30 E-08 \quad 3.42 E-08$

DEFINE CR-51

$\begin{array}{llllll}2.77 E+01 & 3.26 E-02 & 5.01 E-03 & 2.60 E+02 & 1.30 E+02 & 1.00 E+00\end{array}$

OTHER

$\begin{array}{lllll}0.00 E+00 & 1.00 E-02 & 1.00 E+00 & 0.00 E+00 & 0.00 E+00\end{array}$

CULVL 10000000000.00

EOF

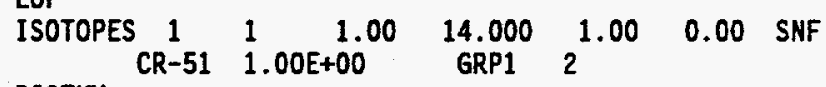

DISTKM

NMODE $=1 \quad 1.00$

PKGSIZ

EOF

SNF $\quad 3.00$ 
RUN DATE: [ 16-APR-95 AT 15:52:45]

PAGE 4

_INCIDENT-FREE_HAND_CHECK_TRUCK_SUBURBAN_

\begin{tabular}{lc}
\multicolumn{1}{c}{ ZONE } & POPULATION DENSITY \\
& (PERSONS PER SQ KM) \\
RURAL & 6. \\
SUBURBAN & 719. \\
URBAN & 3861.
\end{tabular}

PACKAGE CHARACTERISTICS

$\begin{array}{cccc}\text { FOR } & \text { DIMENSION } & \text { EFFECTIVE } & \text { K(0) } \\ \text { MATERIAL } & \text { (METERS) } & \text { DIMENSION } & \text { METERS SQ. } \\ \text { SNF } & 3.000 E+00 & 3.000 E+00 & 6.250 E+00\end{array}$

$K(0)$ IS TI TO DOSE RATE CONVERSION FACTOR

PACKAGE HANDLING THRESHOLDS (METERS)

PKGSZ1 $=5.000 E-01$

PKGSZ2 $=1.000 E+00$

PACKAGES .LE. PKGSZ1 ARE HAND CARRIED

PACKAGES .GT. PKGSZ1 AND .LE. PKGSZ2 ARE HANDLED BY SMALL EQUIPMENT

PACKAGES .GT. PKGSZZ ARE HANDLED BY HEAVY EQUIPMENT

MATERIAL CHARACTERISTICS

$\begin{array}{rrr} & \text { FRACTION } & \text { FRACTION } \\ \text { MATERIAL } & \text { OF GAMMA } & \text { OF NEUTRON } \\ \text { SNF } & 1.000 E+00 & 0.000 E+00\end{array}$


RUN DATE: [ 16-APR-95 AT 15:52:45 ]

_INCIDENT-FREE_HAND_CHECK_TRUCK_SUBURBAN_

MODE CHARACTERISTICS

$\begin{array}{ccccccc}\text { MODE } & \begin{array}{c}\text { DISTANCE } \\ \text { TRAVELED }\end{array} & \begin{array}{c}\text { EXCLUSIVE } \\ \text { USE }\end{array} & \begin{array}{c}\text { NUMBER OF } \\ \text { SHIPMENTS }\end{array} & \text { MATERIALS } & \begin{array}{l}\text { TRANSPORT } \\ \text { INDEX (TI) }\end{array} & \begin{array}{c}\text { PACKAGES/ } \\ \text { SHIPMENT }\end{array} \\ \text { TRUCK } & 1.00 E+00 & \text { NO } & 1.00 E+00 & & & \\ & & & & \text { SNF } & 1.40 E+01 & 1.00 E+00\end{array}$

BUILDING SHIELDING OPTION= 3

(1=TOTAL SHIELDING, 2=PARTIAL SHIELDING, 3=NO SHIELDING)

$R P D=6.000 E+00$

(RATIO OF PEDESTRIAN DENSITY (PEDESTRIAN/KM SQ OF SIDEWALK)

TO POPULATION DENSITY (PEOPLE/KM SQ IN URBAN AREAS)

$R R=1.000 E+00$

(TRANSMISSION FACTOR FOR RURAL AREAS)

RS $=1.000 E+00$

(TRANSMISSION FACTOR FOR SUBURBAN AREAS)

$\mathrm{RU}=1.000 \mathrm{E}+00$

(TRANSMISSION FACTOR FOR URBAN AREAS) 
No

1 FRACTION OF TRAVEL IN RURAL POPULATION ZONE

2 FRACTION OF TRAVEL IN SUBURBAN POPULATION ZONE

3 FRACTION OF TRAVEL IN URBAN POPULATION ZONE

4 VELOCITY IN RURAL POPULATION ZONE (KILOMETERS/HOUR)

5 VELOCITY IN SUBURBAN POP. ZONE 4.025E+01 (KILOMETERS/HOUR)

6 VELOCITY IN URBAN POPULATION ZONE (KILOMETERS/HOUR)

7 NUMBER OF CREWMEN

$2.416 E+01$

$2.000 E+00$

8 DISTANCE FROM SOURCE TO CREW (METERS)

9 NUMBER OF HANDLINGS

$3.100 E+00$

$0.000 E+00$

10 STOP TIME PER KM (HR/KM)

11 MINIMUM STOP TIME PER TRIP (HR)

12 ZERO STOP TIME PER TRIP (HR) $0.000 E+00$

13 MINIMUM NUMBER OF RAIL CLASSIF $0.000 E+00$ ICATIONS/ INSPECTIONS

14 PERSONS EXPOSED WHILE STOPPED 5.000E+01

15 AVERAGE EXPOSURE DISTANCE 2.000E+01 WHILE STOPPED (METERS)

16 STORAGE TIME PER SHIPMENT $0.000 E+00$ (HR)

17 NUMBER OF EXPOSED PERSONS $0.000 E+00$ DURING STORAGE

18 AVERAGE EXPOSURE DISTANCE WHILE IN STORAGE (METERS)

19 NUMBER OF PEOPLE PER VEHICLE ON LINK

20 FRACTION OF URBAN TRAVEL DURING RUSH HOUR TRAFFIC

21 FRACTION OF URBAN TRAVEL ON CITY STREETS

22 FRACTION OF RURAL-SUBURBAN TRAVEL ON FREEWAYS

23 *TRAFFIC COUNT PASSING A SPECIFIC POINT-RURAL ZONE

24 *TRAFFIC COUNT PASSING A SPECIFIC POINT-SUBURBAN ZONE

25 `TRAFFIC COUNT PASSING A SPECIFIC POINT-URBAN ZONE

${ }^{*}$ (ONE WAY VEHICLES/HR)
$1.000 \mathrm{E}+02$

2. $000 E+00$

$1.000 E-01$

$5.000 E-02$

$1.000 E+00$

4.700E+02

$7.800 E+02$

$2.800 E+03$ 
RUN DATE: [ 16-APR-95 AT 15:52:45]

_INCIDENT-FREE_HAND_CHECK_TRUCK_SUBURBAN_

ISOTOPE RELATED DATA

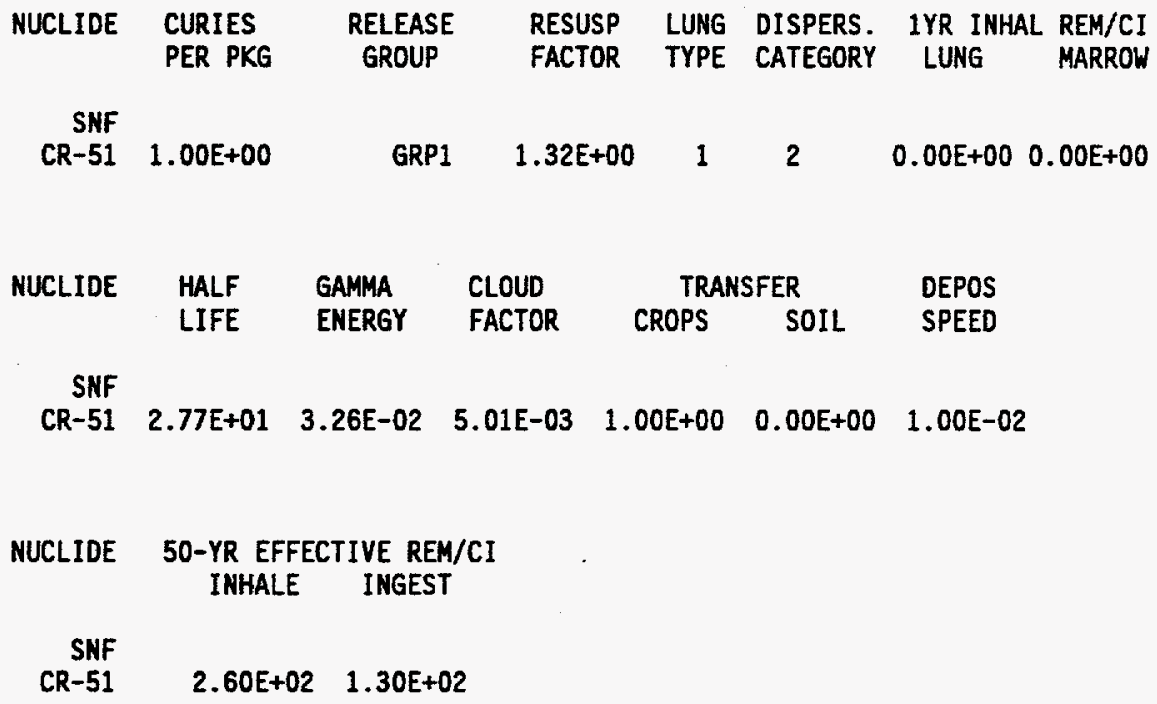


_INCIDENT-FREE_HAND_CHECK_TRUCK_SUBURBAN_

RELEASE RELATED DATA

$\begin{array}{rrrr}\text { ACCIDENT RATES (PER KM) } \\ \text { MODE } & \text { RURAL } & \text { SUBURBAN } & \text { URBAN } \\ \text { TRUCK } & 1.000 \mathrm{E}+00 & 1.000 \mathrm{E}+00 & 1.000 \mathrm{E}+00\end{array}$

RELEASE FRACTIONS

GROUP SEVER: 1 SEVER: 2 SEVER: 3 SEVER: 4 SEVER: 5 SEVER: 6

$1 \quad 1.00 E+00 \quad 1.00 E+00 \quad 1.00 E+00 \quad 1.00 E+00 \quad 1.00 E+00 \quad 1.00 E+00$

ACCIDENT SEVERITY FRACTIONS

FOR TRUCK

$\begin{array}{cllllll}\text { ZONE } & \text { SEVER: } 1 & \text { SEVER: } 2 & \text { SEVER: } 3 & \text { SEVER: } 4 & \text { SEVER: } 5 & \text { SEVER: } 6 \\ 1 & 1.00 E+00 & 0.00 E+00 & 0.00 E+00 & 0.00 E+00 & 0.00 E+00 & 0.00 E+00 \\ 2 & 1.00 E+00 & 0.00 E+00 & 0.00 E+00 & 0.00 E+00 & 0.00 E+00 & 0.00 E+00 \\ 3 & 1.00 E+00 & 0.00 E+00 & 0.00 E+00 & 0.00 E+00 & 0.00 E+00 & 0.00 E+00\end{array}$ 
_INCIDENT-FREE_HAND_CHECK_TRUCK_SUBURBAN_

AEROSOLIZED FRACTION OF RELEASED MATERIAL

$\begin{array}{cllllll}\text { DISP CAT } & \text { SEVER: } 1 & \text { SEVER: 2 } & \text { SEVER: } 3 & \text { SEVER: } 4 & \text { SEVER: } 5 & \text { SEVER: } 6 \\ 1 & 0.00 E+00 & 0.00 E+00 & 0.00 E+00 & 0.00 E+00 & 0.00 E+00 & 0.00 E+00 \\ 2 & 1.00 E+00 & 1.00 E+00 & 1.00 E+00 & 1.00 E+00 & 1.00 E+00 & 1.00 E+00 \\ 3 & 1.00 E-02 & 1.00 E-02 & 1.00 E-02 & 1.00 E-02 & 1.00 E-02 & 1.00 E-02 \\ 4 & 5.00 E-02 & 5.00 E-02 & 5.00 E-02 & 5.00 E-02 & 5.00 E-02 & 5.00 E-02 \\ 5 & 1.00 E-01 & 1.00 E-01 & 1.00 E-01 & 1.00 E-01 & 1.00 E-01 & 1.00 E-01 \\ 6 & 1.00 E+00 & 1.00 E+00 & 1.00 E+00 & 1.00 E+00 & 1.00 E+00 & 1.00 E+00 \\ 7 & 1.00 E+00 & 1.00 E+00 & 1.00 E+00 & 1.00 E+00 & 1.00 E+00 & 1.00 E+00 \\ 8 & 1.00 E+00 & 1.00 E+00 & 1.00 E+00 & 1.00 E+00 & 1.00 E+00 & 1.00 E+00 \\ 9 & 1.00 E+00 & 1.00 E+00 & 1.00 E+00 & 1.00 E+00 & 1.00 E+00 & 1.00 E+00 \\ 10 & 1.00 E+00 & 1.00 E+00 & 1.00 E+00 & 1.00 E+00 & 1.00 E+00 & 1.00 E+00 \\ 11 & 1.00 E+00 & 1.00 E+00 & 1.00 E+00 & 1.00 E+00 & 1.00 E+00 & 1.00 E+00\end{array}$

FRACTION OF AEROSOLS BELOW 10 MICRONS AED

$\begin{array}{ccccccc}\text { DISP CAT } & \text { SEVER: } 1 & \text { SEVER: } 2 & \text { SEVER: } 3 & \text { SEVER: } 4 & \text { SEVER: } 5 & \text { SEVER: } 6 \\ 1 & 0.00 E+00 & 0.00 E+00 & 0.00 E+00 & 0.00 E+00 & 0.00 E+00 & 0.00 E+00 \\ 2 & 1.00 E+00 & 1.00 E+00 & 1.00 E+00 & 1.00 E+00 & 1.00 E+00 & 1.00 E+00 \\ 3 & 5.00 E-02 & 5.00 E-02 & 5.00 E-02 & 5.00 E-02 & 5.00 E-02 & 5.00 E-02 \\ 4 & 5.00 E-02 & 5.00 E-02 & 5.00 E-02 & 5.00 E-02 & 5.00 E-02 & 5.00 E-02 \\ 5 & 5.00 E-02 & 5.00 E-02 & 5.00 E-02 & 5.00 E-02 & 5.00 E-02 & 5.00 E-02 \\ 6 & 5.00 E-02 & 5.00 E-02 & 5.00 E-02 & 5.00 E-02 & 5.00 E-02 & 5.00 E-02 \\ 7 & 1.00 E+00 & 1.00 E+00 & 1.00 E+00 & 1.00 E+00 & 1.00 E+00 & 1.00 E+00 \\ 8 & 1.00 E+00 & 1.00 E+00 & 1.00 E+00 & 1.00 E+00 & 1.00 E+00 & 1.00 E+00 \\ 9 & 1.00 E+00 & 1.00 E+00 & 1.00 E+00 & 1.00 E+00 & 1.00 E+00 & 1.00 E+00 \\ 10 & 1.00 E+00 & 1.00 E+00 & 1.00 E+00 & 1.00 E+00 & 1.00 E+00 & 1.00 E+00 \\ 11 & 1.00 E+00 & 1.00 E+00 & 1.00 E+00 & 1.00 E+00 & 1.00 E+00 & 1.00 E+00\end{array}$


RUN DATE: [ 16-APR-95 AT 15:52:45]

_INCIDENT-FREE_HAND_CHECK_TRUCK_SUBURBAN_

COST RELATED DATA

EMERGENCY RESPONSE COST

SEVER: 1 SEVER: 2 SEVER: 3 SEVER: 4 SEVER: 5 SEVER: 6

ON-SCENE COSTS

(RF=RELEASE FRACTION)

$R F=0$. $\quad 0 . \quad 0 .<R F<=.01$ o. $\quad .01<R F<=0.1$ 0. $\quad .1<R F<=1$

0 . 
RUN DATE: [ 16-APR-95 AT 15:52:45 ]

_INCIDENT-FREE_HAND_CHECK_TRUCK_SUBURBAN_

HEALTH RELATED DATA

EARLY. FATALITY PROBABILITIES

$\begin{array}{rrrrr}\text { DOSE (REM) } & \text { LUNG-1 } & \text { LUNG }-2 & \text { LUNG } 3 & \text { MARROW } \\ 100000.000 & 1.000 E+00 & 1.000 E+00 & 1.000 E+00 & 1.000 E+00 \\ 80000.000 & 1.000 E+00 & 8.500 E-01 & 8.000 E-01 & 1.000 E+00 \\ 70000.000 & 1.000 E+00 & 8.000 E-01 & 5.000 E-01 & 1.000 E+00 \\ 40000.000 & 1.000 E+00 & 7.000 E-01 & 0.000 E+00 & 1.000 E+00 \\ 30000.000 & 1.000 E+00 & 5.000 E-01 & 0.000 E+00 & 1.000 E+00 \\ 25000.000 & 1.000 E+00 & 2.000 E-01 & 0.000 E+00 & 1.000 E+00 \\ 20000.000 & 1.000 E+00 & 8.000 E-02 & 0.000 E+00 & 1.000 E+00 \\ 10000.000 & 6.000 E-01 & 0.000 E+00 & 0.000 E+00 & 1.000 E+00 \\ 8000.000 & 1.000 E-01 & 0.000 E+00 & 0.000 E+00 & 1.000 E+00 \\ 6000.000 & 6.000 E-02 & 0.000 E+00 & 0.000 E+00 & 1.000 E+00 \\ 4000.000 & 3.000 E-02 & 0.000 E+00 & 0.000 E+00 & 1.000 E+00 \\ 3000.000 & 0.000 E+00 & 0.000 E+00 & 0.000 E+00 & 1.000 E+00 \\ 2000.000 & 0.000 E+00 & 0.000 E+00 & 0.000 E+00 & 1.000 E+00 \\ 1000.000 & 0.000 E+00 & 0.000 E+00 & 0.000 E+00 & 1.000 E+00 \\ 800.000 & 0.000 E+00 & 0.000 E+00 & 0.000 E+00 & 9.960 E-01 \\ 700.000 & 0.000 E+00 & 0.000 E+00 & 0.000 E+00 & 9.000 E-01 \\ 600.000 & 0.000 E+00 & 0.000 E+00 & 0.000 E+00 & 4.000 E-01 \\ 500.000 & 0.000 E+00 & 0.000 E+00 & 0.000 E+00 & 5.000 E-02 \\ 400.000 & 0.000 E+00 & 0.000 E+00 & 0.000 E+00 & 0.000 E+00 \\ 300.000 & 0.000 E+00 & 0.000 E+00 & 0.000 E+00 & 0.000 E+00 \\ 100.000 & 0.000 E+00 & 0.000 E+00 & 0.000 E+00 & 0.000 E+00 \\ 75.000 & 0.000 E+00 & 0.000 E+00 & 0.000 E+00 & 0.000 E+00 \\ 50.000 & 0.000 E+00 & 0.000 E+00 & 0.000 E+00 & 0.000 E+00 \\ 30.000 & 0.000 E+00 & 0.000 E+00 & 0.000 E+00 & 0.000 E+00 \\ 15.000 & 0.000 E+00 & 0.000 E+00 & 0.000 E+00 & 0.000 E+00 \\ 5.000 & 0.000 E+00 & 0.000 E+00 & 0.000 E+00 & 0.000 E+00 \\ 1.000 & 0.000 E+00 & 0.000 E+00 & 0.000 E+00 & 0.000 E+00 \\ 0.100 & 0.000 E+00 & 0.000 E+00 & 0.000 E+00 & 0.000 E+00 \\ 0.010 & 0.000 E+00 & 0.000 E+00 & 0.000 E+00 & 0.000 E+00 \\ 0.010 & 0.000 E+00 & 0.000 E+00 & 0.000 E+00 & 0.000 E+00\end{array}$


RUN DATE: [ 16-APR-95 AT 15:52:45]

_INCIDENT-FREE_HAND_CHECK_TRUCK_SUBURBAN_

DISPERSAL ACCIDENT INPUT

$\begin{array}{cc}\begin{array}{c}\text { AREADA } \\ \text { (N SQ) }\end{array} & \begin{array}{c}\text { DILUTION } \\ \text { FACTOR* } \\ 4.590 E+02\end{array} \\ 1.530 E+03 & 3.420 E-03 \\ 3.940 E+03 & 1.720 E-03 \\ 1.250 E+04 & 8.580 E-04 \\ 3.040 E+04 & 3.420 E-04 \\ 6.850 E+04 & 1.720 E-04 \\ 1.760 E+05 & 8.580 E-05 \\ 4.450 E+05 & 3.420 E-05 \\ 8.590 E+05 & 1.720 E-05 \\ 2.550 E+05 & 8.580 E-06 \\ 4.450 E+06 & 3.420 E-06 \\ 1.030 E+07 & 1.720 E-06 \\ 2.160 E+07 & 8.580 E-07 \\ 5.520 E+07 & 3.420 E-07 \\ 1.770 E+08 & 1.720 E-07 \\ 4.890 E+08 & 8.580 E-08 \\ 8.120 E+08 & 4.420 E-08 \\ 1.350 E+09 & 3.300 E-08 \\ & 3.420 E-08\end{array}$

* DILUTION FACTOR UNITS ARE (CI-SEC/M**3/CI-RELEASED)

NON-DISPERSAL ACCIDENT INPUT

$\begin{array}{lcl} & \text { RADIST(M) } \\ \text { RURAL } & \text { SUBURBAN } & \text { URBAN } \\ 3.050 E+00 & 3.050 E+00 & 3.050 E+00 \\ 6.100 E+00 & 6.100 E+00 & 6.100 E+00 \\ 9.100 E+00 & 9.100 E+00 & 9.100 E+00 \\ 1.220 E+01 & 1.220 E+01 & 1.220 E+01 \\ 1.520 E+01 & 1.520 E+01 & 1.520 E+01 \\ 3.050 E+01 & 3.050 E+01 & 3.050 E+01 \\ 6.100 E+01 & 6.100 E+01 & 6.100 E+01 \\ 9.140 E+01 & 9.140 E+01 & 9.140 E+01 \\ 1.524 E+02 & 1.524 E+02 & 1.524 E+02 \\ 3.050 E+02 & 3.050 E+02 & 3.050 E+02\end{array}$

BUILDING DOSE FACTOR

FRACTION OF LAND UNDER CULTIVATION

CONTANINATION CLEAN UP LEVEL (UCI/ $/{ }^{* *} 2$ ) BREATHING RATE ( $M{ }^{\star \star} 3 /$ SEC)
$=8.600 \mathrm{E}-03$

$=4.400 \mathrm{E}-01$

$=1.000 E+09$

$=3.300 \mathrm{E}-04$ 
_INCIDENT-FREE_HAND_CHECK_TRUCK_SUBURBAN_

\section{REGULATORY CHECKS}

MODE 1 HAS BEEN REDESIGNATED AS EXCLUSIVE USE

FOR THE SHIPMENT OF

SNF BY MODE 1

THE DOSE RATE AT 2 METERS COULD EXCEED 10 MR/HR

PPS*TI HAS BEEN RESET TO EQUAL 14.00

FOR THE SHIPMENT OF

SNF BY MODE 1

THE DOSE RATE IN THE CREW COMPARTMENT COULD EXCEED 2 MREM/HR

THE DOSE RATE HAS BEEN RESET FROM 9.11 TO 2 FOR CREW CALCULATIONS 
_INCIDENT-FREE_HAND_CHECK_TRUCK_SUBURBAN_

INCIDENT-FREE SUMMARY

INCIDENT-FREE POPULATION EXPOSURE IN PERSON-REM

PASSENGR CREW HANDLERS OFF LINK ON LINK STOPS STORAGE TOTALS LINK $10.00 E+00 \quad 1.00 E-04 \quad 0.00 E+00 \quad 1.63 E-05 \quad 1.45 E-05 \quad 1.20 E-04 \quad 0.00 E+00 \quad 2.51 E-04$

TOTALS: $0.00 E+00 \quad 1.00 E-04 \quad 0.00 E+00 \quad 1.63 E-05 \quad 1.45 E-05 \quad 1.20 E-04 \quad 0.00 E+00 \quad 2.51 E-04$

MAXIMUM INDIVIDUAL IN-TRANSIT DOSE

LINK 1 3.34E-07 REM 
RUN DATE: [ 16-APR-95 AT 15:52:45 ]

_INCIDENT-FREE_HAND_CHECK_TRUCK_SUBURBAN_

\begin{tabular}{|c|c|c|}
\hline \multicolumn{3}{|c|}{ 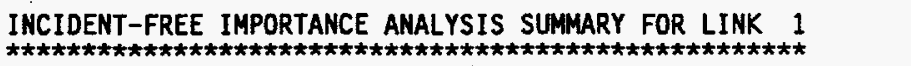 } \\
\hline IDEX & DESCRIPTION OF PARAMETER & IMPORTANCE \\
\hline $\begin{array}{r}1 \\
2 \\
3 \\
4 \\
5 \\
6 \\
7 \\
8 \\
9 \\
10 \\
11 \\
12 \\
13 \\
14 \\
15 \\
16 \\
17 \\
18 \\
19 \\
20 \\
21 \\
22 \\
23 \\
24 \\
25 \\
26 \\
27 \\
28 \\
29 \\
30 \\
31 \\
32 \\
33 \\
34 \\
35 \\
36 \\
37 \\
38\end{array}$ & 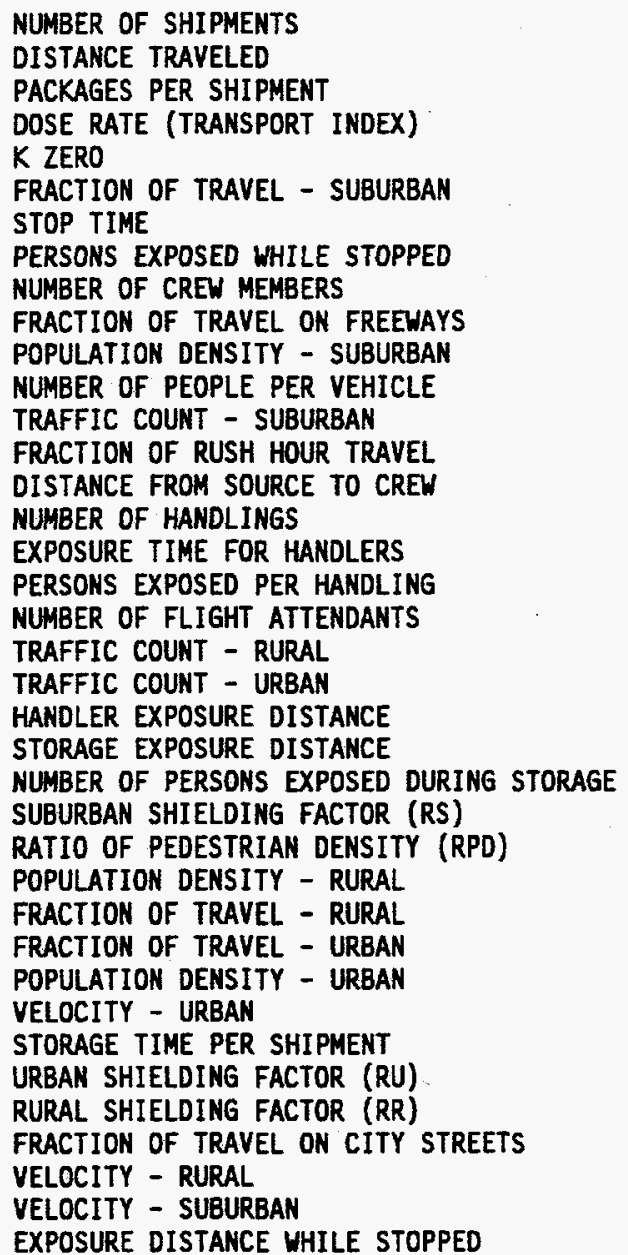 & $\begin{array}{r}2.512 E-06 \\
2.512 E-06 \\
1.510 E-06 \\
1.510 E-06 \\
1.510 E-06 \\
1.309 E-06 \\
1.203 E-06 \\
1.203 E-06 \\
1.002 E-06 \\
3.073 E-07 \\
1.626 E-07 \\
1.447 E-07 \\
1.447 E-07 \\
7.604 E-08 \\
0.000 E+00 \\
0.000 E+00 \\
0.000 E+00 \\
0.000 E+00 \\
0.000 E+00 \\
0.000 E+00 \\
0.000 E+00 \\
0.000 E+00 \\
0.000 E+00 \\
0.000 E+00 \\
0.000 E+00 \\
0.000 E+00 \\
0.000 E+00 \\
0.000 E+00 \\
0.000 E+00 \\
0.000 E+00 \\
0.000 E+00 \\
0.000 E+00 \\
0.000 E+00 \\
0.000 E+00 \\
0.000 E+00 \\
-4.521 E-00 \\
-1.002 E-06 \\
-2.406 E-06\end{array}$ \\
\hline
\end{tabular}

THE IMPORTANCE VALUE ESTIMATES THE PERSON-REM INFLUENCE OF A ONE PERCENT INCREASE IN THE PARAMETER

TOTAL EXPOSED POPULATION: INCIDENT-FREE

$\begin{array}{ll}\text { TRUCK RURAL } & 0.00 E+00 \text { PERSONS } \\ \text { TRUCK SUBURBAN } & 1.15 E+03 \text { PERSONS } \\ \text { TRUCK URBAN } & 0.00 E+00 \text { PERSONS }\end{array}$

TOTAL 1.15E+03 PERSONS

TOTAL EXPOSED POPULATION: ACCIDENT

(PERSONS UNDER PLUME FOOTPRINT FOR A SINGLE ACCIDENT)

EOI
TRUCK RURAL
$0.00 E+00$ PERSONS
TRUCK SUBURBAN
$0.00 E+00$ PERSONS
TRUCK URBAN
$0.00 E+00$ PERSONS

END OF RUN 


\section{A.3 TURFU.OUT}

UNIT RISK FACTORS FOR TRUCKS IN URBAN POPULATION ZONE 


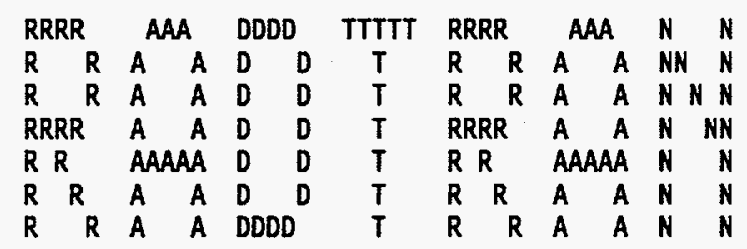

RADTRAN 4.0.17 VERSION DATE: NOVEMBER 8, 1994

MODE DESCRIPTIONS

$\begin{array}{cll}\text { NUMBER } & \text { NAME } & \text { CHARACTERIZATION } \\ 1 & \text { TRUCK } & \text { LONG HAUL VEHICLE } \\ 2 & \text { RAIL } & \text { COMMERCIAL TRAIN } \\ 3 & \text { BARGE } & \text { INLAND VESSEL } \\ 4 & \text { SHIP } & \text { OPEN SEA VESSEL } \\ 5 & \text { CARGO AIR } & \text { CARGO AIRCRAFT } \\ 6 & \text { PASS AIR } & \text { PASSENGER AIRCRAFT } \\ 7 & \text { P-VAN } & \text { PASSENGER VAN } \\ 8 & \text { CVAN-T } & \text { COMMERCIAL VAN } \\ 9 & \text { CVAN-R } & \text { COMMERCIAL VAN } \\ 10 & \text { CVAN-CA } & \text { COMMERCIAL VAN }\end{array}$




\section{ECHO CHECK}

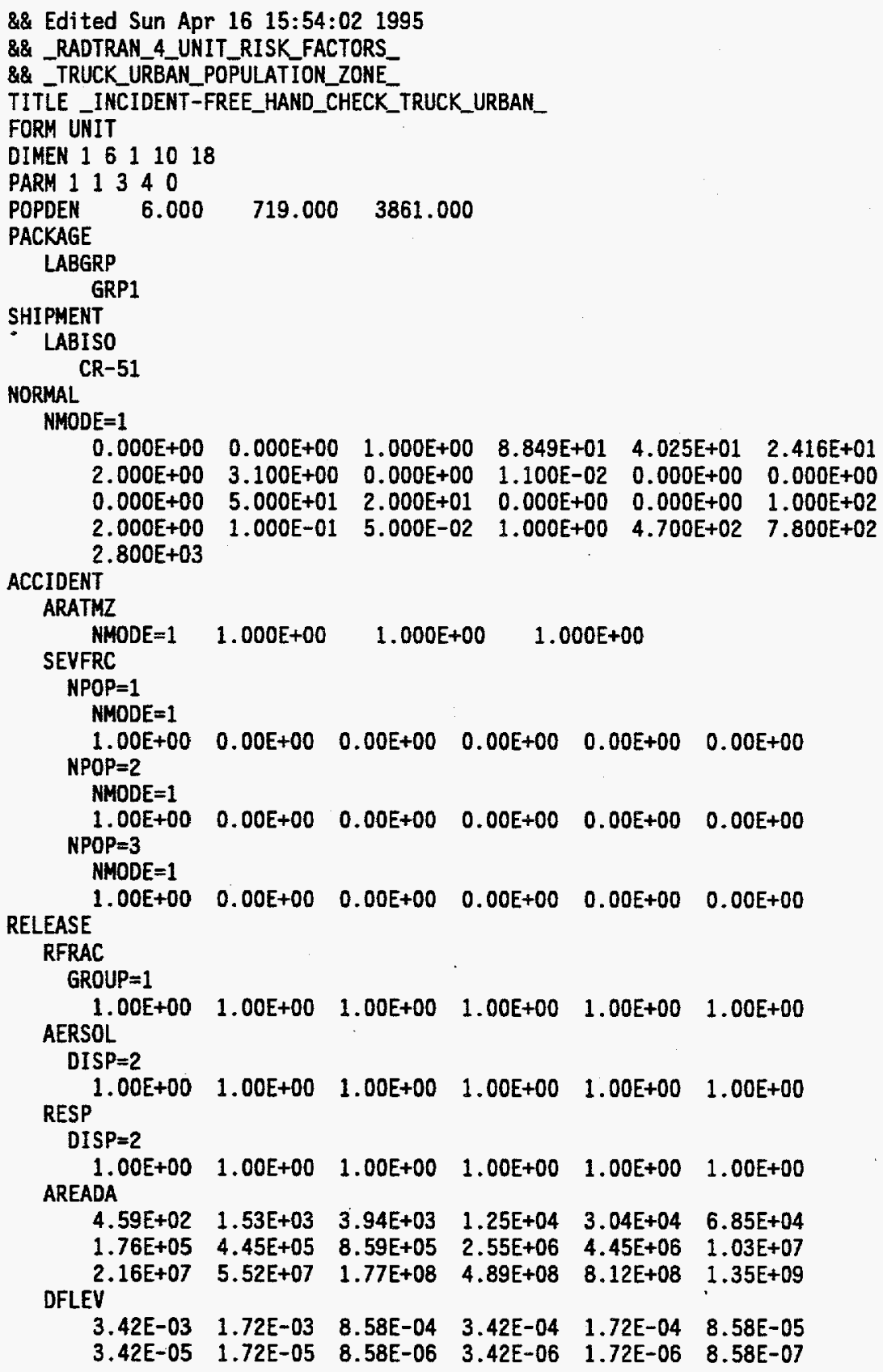


RUN DATE: [ 16-APR-95 AT 15:54:34 ]

_INCIDENT-FREE_HAND_CHECK_TRUCK_URBAN_

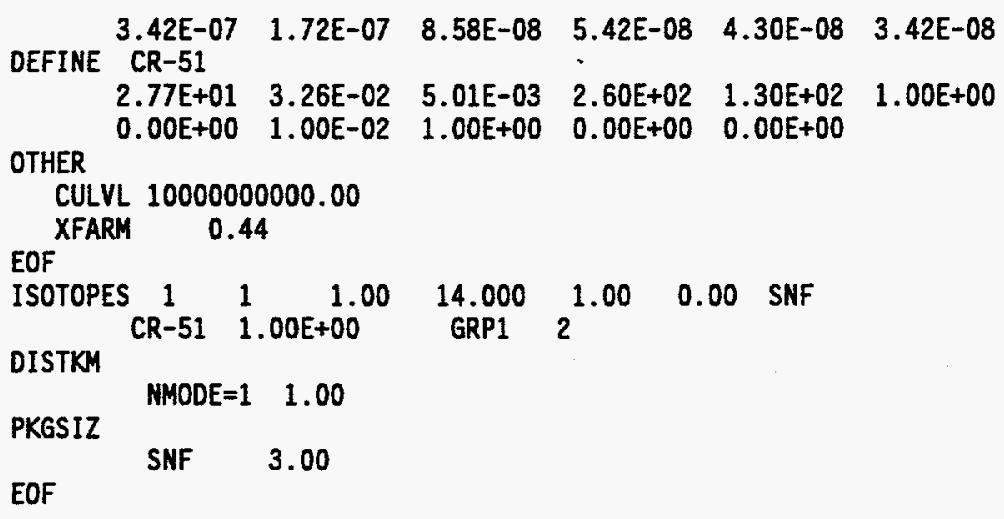




\begin{tabular}{lc}
\multicolumn{1}{c}{ ZONE } & POPULATION DENSITY \\
& (PERSONS PER SQ KM) \\
RURAL & 6. \\
SUBURBAN & 719. \\
URBAN & 3861.
\end{tabular}

PACKAGE CHARACTERISTICS

$\begin{array}{cccc}\text { FOR } & \text { DIMENSION } & \text { EFFECTIVE } & \text { K(0) } \\ \text { MATERIAL } & \text { (METERS) } & \text { DIMENSION } & \text { METERS SQ. } \\ \text { SNF } & 3.000 E+00 & 3.000 E+00 & 6.250 E+00\end{array}$

$K(0)$ IS TI TO DOSE RATE CONVERSION FACTOR

PACKAGE HANDLING THRESHOLDS (METERS)

PKGSZ1 $=5.000 E-01$

PKGSZ2 $=1.000 E+00$

PACKAGES . LE. PKGSZ1 ARE HAND CARRIED

PACKAGES .GT. PKGSZI AND .LE. PKGSZ2 ARE HANDLED BY SMALL EQUIPMENT

PACKAGES .GT. PKGSZ2 ARE HANDLED BY HEAVY EQUIPMENT

\begin{tabular}{rrr}
\multicolumn{3}{c}{ MATERIAL CHARACTERISTICS } \\
& & \\
MATERIAL & FRACTION & FRACTION \\
SNF & $1.000 E+00$ & $0.000 E+00$
\end{tabular}


RUN DATE: [ 16-APR-95 AT 15:54:34 ]

_INCIDENT-FREE_HAND_CHECK_TRUCK_URBAN_

MODE CHARACTERISTICS

$\begin{array}{ccccccc}\text { MODE } & \begin{array}{c}\text { DISTANCE } \\ \text { TRAVELED }\end{array} & \begin{array}{c}\text { EXCLUSIVE } \\ \text { USE }\end{array} & \begin{array}{c}\text { NUMBER OF } \\ \text { SHIPMENTS }\end{array} & \text { MATERIALS } & \begin{array}{c}\text { TRANSPORT } \\ \text { INDEX (TI) }\end{array} & \begin{array}{c}\text { PACKAGES/ } \\ \text { SHIPMENT }\end{array} \\ \text { TRUCK } & 1.00 E+00 & \text { NO } & 1.00 E+00 & & & \\ & & & & \text { SNF } & 1.40 E+01 & 1.00 E+00\end{array}$

BUILDING SHIELDING OPTION $=3$

(1=TOTAL SHIELDING, 2=PARTIAL SHIELDING, 3=NO SHIELDING)

$R P D=6.000 E+00$

(RATIO OF PEDESTRIAN DENSITY (PEDESTRIAN/KM SQ OF SIDEWALK)

TO POPULATION DENSITY (PEOPLE/KM SQ IN URBAN AREAS)

$R R=1.000 E+00$

(TRANSMISSION FACTOR FOR RURAL AREAS)

RS $=1.000 E+00$

(TRANSHISSION FACTOR FOR SUBURBAN AREAS)

$R U=1.000 E+00$

(TRANSMISSION FACTOR FOR URBAN AREAS) 
NO

1 FRACTION OF TRAVEL IN RURAL POPULATION ZONE

2 FRACTION OF TRAVEL IN SUBURBAN POPULATION ZONE

3 FRACTION OF TRAVEL IN URBAN POPULATION ZONE

4 VELOCITY IN RURAL POPULATION ZONE (KILOMETERS/HOUR)

5 VELOCITY IN SUBURBAN POP. ZONE 4.025E+01 (KILOMETERS/HOUR)

6 VELOCITY IN URBAN POPULATION 2.416E+01 ZONE (KILOMETERS/HOUR)

7 NUMBER OF CREMMEN

$2.000 E+00$

8 DISTANCE FROM SOURCE TO CREW 3.100E+00 (METERS)

9 NUMBER OF HANDLINGS

$0.000 E+00$

10 STOP TIME PER KM (HR/KM)

1.100E-02

11 MINIMUM STOP TIME PER TRLP (HR)

12 ZERO STOP TIME PER TRIP (HR)

$0.000 E+00$

$0.000 E+00$

13 MINIMUM NUMBER OF RAIL CLASSIF 0.000E+00 ICATIONS/INSPECTIONS

14 PERSONS EXPOSED WHILE STOPPED 5.000E+01

15 AVERAGE EXPOSURE DISTANCE WHILE STOPPED (METERS)

16 STORAGE TIME PER SHIPMENT (HR)

17 NUMBER OF EXPOSED PERSONS DURING STORAGE

18 AVERAGE EXPOSURE DISTANCE WHILE IN STORAGE (METERS)

19 NUMBER OF PEOPLE PER VEHICLE ON LINK

20 FRACTION OF URBAN TRAVEL DURING RUSH HOUR TRAFFIC

21 FRACTION OF URBAN TRAVEL ON CITY STREETS

22 FRACTION OF RURAL-SUBURBAN TRAVEL ON FREEWAYS

23 *TRAFFIC COUNT PASSING A SPECIFIC POINT-RURAL ZONE

24 `TRAFFIC COUNT PASSING A SPECIFIC POINT-SUBURBAN ZONE

25 *TRAFFIC COUNT PASSING A SPECIFIC POINT-URBAN ZONE

* (ONE WAY VEHICLES/HR)
$2.000 E+01$

$0.000 E+00$

$0.000 E+00$

$1.000 E+02$

$2.000 E+00$

1.000E-01

5.000E-02

$4.700 E+02$

$7.800 E+02$

$2.800 E+03$
$1.000 E+00$ 
RUN DATE: [ 16-APR-95 AT 15:54:34 ]

PAGE 7

_INCIDENT-FREE_HAND_CHECK_TRUCK_URBAN_

ISOTOPE RELATED DATA

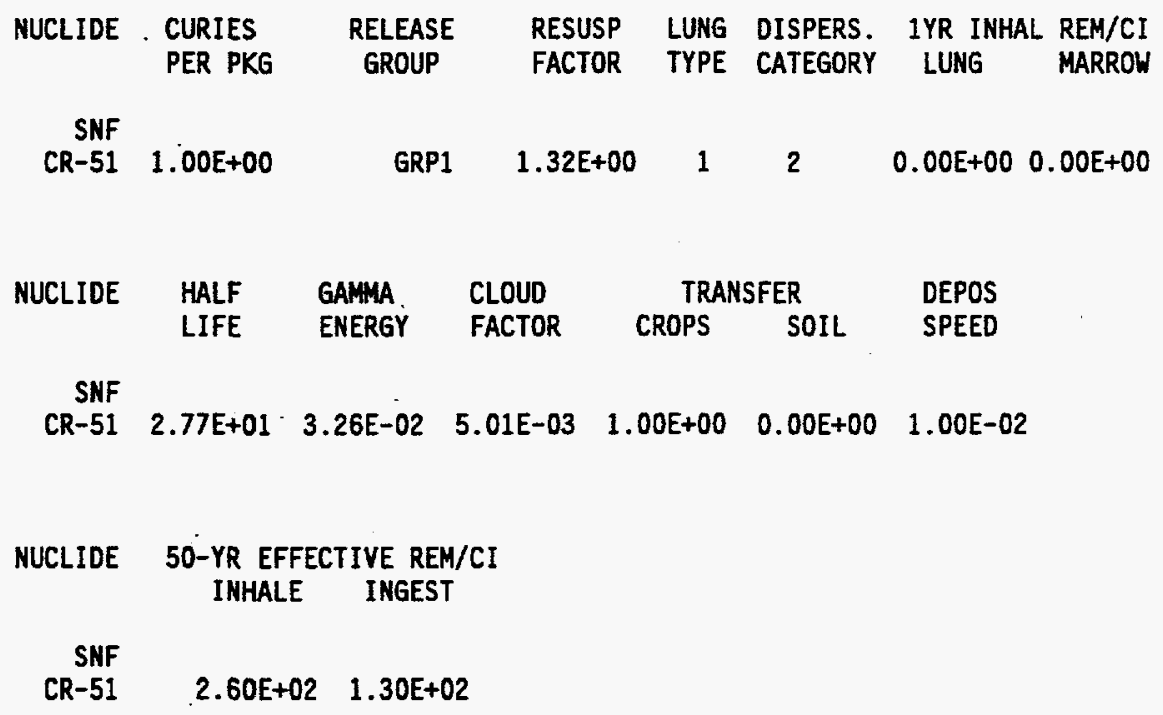


RUN DATE: [ 16-APR-95 AT 15:54:34 ]

_INCIOENT-FREE_HAND_CHECK_TRUCK_URBAN_

RELEASE RELATED DATA

ACCIDENT RATES (PER KM)

MODE RURAL SUBURBAN URBAN

TRUCK $1.000 E+00 \quad 1.000 E+00 \quad 1.000 E+00$

RELEASE FRACTIONS

GROUP SEVER: 1 SEVER: 2 SEVER: 3 SEVER: 4 SEVER: 5 SEVER: 6

$11.00 E+00 \quad 1.00 E+00 \quad 1.00 E+00 \quad 1.00 E+00 \quad 1.00 E+00 \quad 1.00 E+00$

ACCIDENT SEVERITY FRACTIONS

FOR TRUCK

$\begin{array}{cllllll}\text { ZONE } & \text { SEVER: 1 } & \text { SEVER: } 2 & \text { SEVER: } 3 & \text { SEVER: } 4 & \text { SEVER: } 5 & \text { SEVER: } 6 \\ 1 & 1.00 E+00 & 0.00 E+00 & 0.00 E+00 & 0.00 E+00 & 0.00 E+00 & 0.00 E+00 \\ 2 & 1.00 E+00 & 0.00 E+00 & 0.00 E+00 & 0.00 E+00 & 0.00 E+00 & 0.00 E+00 \\ 3 & 1.00 E+00 & 0.00 E+00 & 0.00 E+00 & 0.00 E+00 & 0.00 E+00 & 0.00 E+00\end{array}$


_INCIDENT-FREE_HAND_CHECK_TRUCK_URBAN_

AEROSOLIZEO FRACTION OF RELEASED MATERIAL

$\begin{array}{cllllll}\text { DISP CAT } & \text { SEVER: } 1 & \text { SEVER: } 2 & \text { SEVER: } 3 & \text { SEVER: } 4 & \text { SEVER: } 5 & \text { SEVER: } 6 \\ 1 & 0.00 E+00 & 0.00 E+00 & 0.00 E+00 & 0.00 E+00 & 0.00 E+00 & 0.00 E+00 \\ 2 & 1.00 E+00 & 1.00 E+00 & 1.00 E+00 & 1.00 E+00 & 1.00 E+00 & 1.00 E+00 \\ 3 & 1.00 E-02 & 1.00 E-02 & 1.00 E-02 & 1.00 E-02 & 1.00 E-02 & 1.00 E-02 \\ 4 & 5.00 E-02 & 5.00 E-02 & 5.00 E-02 & 5.00 E-02 & 5.00 E-02 & 5.00 E-02 \\ 5 & 1.00 E-01 & 1.00 E-01 & 1.00 E-01 & 1.00 E-01 & 1.00 E-01 & 1.00 E-01 \\ 6 & 1.00 E+00 & 1.00 E+00 & 1.00 E+00 & 1.00 E+00 & 1.00 E+00 & 1.00 E+00 \\ 7 & 1.00 E+00 & 1.00 E+00 & 1.00 E+00 & 1.00 E+00 & 1.00 E+00 & 1.00 E+00 \\ 8 & 1.00 E+00 & 1.00 E+00 & 1.00 E+00 & 1.00 E+00 & 1.00 E+00 & 1.00 E+00 \\ 9 & 1.00 E+00 & 1.00 E+00 & 1.00 E+00 & 1.00 E+00 & 1.00 E+00 & 1.00 E+00 \\ 10 & 1.00 E+00 & 1.00 E+00 & 1.00 E+00 & 1.00 E+00 & 1.00 E+00 & 1.00 E+00 \\ 11 & 1.00 E+00 & 1.00 E+00 & 1.00 E+00 & 1.00 E+00 & 1.00 E+00 & 1.00 E+00\end{array}$

FRACTION OF AEROSOLS BELOW 10 MICRONS AED

$\begin{array}{cllllll}\text { DISP CAT } & \text { SEVER: } 1 & \text { SEVER: } 2 & \text { SEVER: } 3 & \text { SEVER: } 4 & \text { SEVER: } 5 & \text { SEVER: } 6 \\ 1 & 0.00 E+00 & 0.00 E+00 & 0.00 E+00 & 0.00 E+00 & 0.00 E+00 & 0.00 E+00 \\ 2 & 1.00 E+00 & 1.00 E+00 & 1.00 E+00 & 1.00 E+00 & 1.00 E+00 & 1.00 E+00 \\ 3 & 5.00 E-02 & 5.00 E-02 & 5.00 E-02 & 5.00 E-02 & 5.00 E-02 & 5.00 E-02 \\ 4 & 5.00 E-02 & 5.00 E-02 & 5.00 E-02 & 5.00 E-02 & 5.00 E-02 & 5.00 E-02 \\ 5 & 5.00 E-02 & 5.00 E-02 & 5.00 E-02 & 5.00 E-02 & 5.00 E-02 & 5.00 E-02 \\ 6 & 5.00 E-02 & 5.00 E-02 & 5.00 E-02 & 5.00 E-02 & 5.00 E-02 & 5.00 E-02 \\ 7 & 1.00 E+00 & 1.00 E+00 & 1.00 E+00 & 1.00 E+00 & 1.00 E+00 & 1.00 E+00 \\ 8 & 1.00 E+00 & 1.00 E+00 & 1.00 E+00 & 1.00 E+00 & 1.00 E+00 & 1.00 E+00 \\ 9 & 1.00 E+00 & 1.00 E+00 & 1.00 E+00 & 1.00 E+00 & 1.00 E+00 & 1.00 E+00 \\ 10 & 1.00 E+00 & 1.00 E+00 & 1.00 E+00 & 1.00 E+00 & 1.00 E+00 & 1.00 E+00 \\ 11 & 1.00 E+00 & 1.00 E+00 & 1.00 E+00 & 1.00 E+00 & 1.00 E+00 & 1.00 E+00\end{array}$


RUN DATE: [ 16-APR-95 AT 15:54:34 ]

_INCIDENT-FREE_HAND_CHECK_TRUCK_URBAN_

COST RELATED DATA

EMERGENCY RESPONSE COST

SEVER: 1 SEVER: 2 SEVER: 3 SEVER: 4 SEVER: 5 SEVER: 6

1 $0.00 E+00 \quad 0.00 E+00 \quad 0.00 E+00 \quad 0.00 E+00 \quad 0.00 E+00 \quad 0.00 E+00$

ON-SCENE COSTS

(RF=RELEASE FRACTION)

$R F=0 . \quad \begin{array}{rrrrr}0 .<R F<=.01 & & .01<R F<=0.1 & .1<R F<=1 . \\ & & 0 . & 0 . & \end{array}$ 
RUN DATE: [ 16-APR-95 AT 15:54:34]

_INCIDENT-FREE_HAND_CHECK_TRUCK_URBAN_

HEALTH RELATED DATA

\section{EARLY FATALITY PROBABILITIES}

$\begin{array}{rrrrr}\text { DOSE (REM) } & \text { LUNG-1 } & \text { LUNG-2 } & \text { LUNG-3 } & \text { MARROW } \\ 100000.000 & 1.000 E+00 & 1.000 E+00 & 1.000 E+00 & 1.000 E+00 \\ 80000.000 & 1.000 E+00 & 8.500 E-01 & 8.000 E-01 & 1.000 E+00 \\ 70000.000 & 1.000 E+00 & 8.000 E-01 & 5.000 E-01 & 1.000 E+00 \\ 40000.000 & 1.000 E+00 & 7.000 E-01 & 0.000 E+00 & 1.000 E+00 \\ 30000.000 & 1.000 E+00 & 5.000 E-01 & 0.000 E+00 & 1.000 E+00 \\ 25000.000 & 1.000 E+00 & 2.000 E-01 & 0.000 E+00 & 1.000 E+00 \\ 20000.000 & 1.000 E+00 & 8.000 E-02 & 0.000 E+00 & 1.000 E+00 \\ 10000.000 & 6.000 E-01 & 0.000 E+00 & 0.000 E+00 & 1.000 E+00 \\ 8000.000 & 1.000 E-01 & 0.000 E+00 & 0.000 E+00 & 1.000 E+00 \\ 6000.000 & 6.000 E-02 & 0.000 E+00 & 0.000 E+00 & 1.000 E+00 \\ 4000.000 & 3.000 E-02 & 0.000 E+00 & 0.000 E+00 & 1.000 E+00 \\ 3000.000 & 0.000 E+00 & 0.000 E+00 & 0.000 E+00 & 1.000 E+00 \\ 2000.000 & 0.000 E+00 & 0.000 E+00 & 0.000 E+00 & 1.000 E+00 \\ 1000.000 & 0.000 E+00 & 0.000 E+00 & 0.000 E+00 & 1.000 E+00 \\ 800.000 & 0.000 E+00 & 0.000 E+00 & 0.000 E+00 & 9.960 E-01 \\ 700.000 & 0.000 E+00 & 0.000 E+00 & 0.000 E+00 & 9.000 E-01 \\ 600.000 & 0.000 E+00 & 0.000 E+00 & 0.000 E+00 & 4.000 E-01 \\ 500.000 & 0.000 E+00 & 0.000 E+00 & 0.000 E+00 & 5.000 E-02 \\ 400.000 & 0.000 E+00 & 0.000 E+00 & 0.000 E+00 & 0.000 E+00 \\ 300.000 & 0.000 E+00 & 0.000 E+00 & 0.000 E+00 & 0.000 E+00 \\ 100.000 & 0.000 E+00 & 0.000 E+00 & 0.000 E+00 & 0.000 E+00 \\ 75.000 & 0.000 E+00 & 0.000 E+00 & 0.000 E+00 & 0.000 E+00 \\ 50.000 & 0.000 E+00 & 0.000 E+00 & 0.000 E+00 & 0.000 E+00 \\ 30.000 & 0.000 E+00 & 0.000 E+00 & 0.000 E+00 & 0.000 E+00 \\ 15.000 & 0.000 E+00 & 0.000 E+00 & 0.000 E+00 & 0.000 E+00 \\ 5.000 & 0.000 E+00 & 0.000 E+00 & 0.000 E+00 & 0.000 E+00 \\ 1.000 & 0.000 E+00 & 0.000 E+00 & 0.000 E+00 & 0.000 E+00 \\ 0.100 & 0.000 E+00 & 0.000 E+00 & 0.000 E+00 & 0.000 E+00 \\ 0.010 & 0.000 E+00 & 0.000 E+00 & 0.000 E+00 & 0.000 E+00 \\ 0.010 & 0.000 E+00 & 0.000 E+00 & 0.000 E+00 & 0.000 E+00\end{array}$


_INCIDENT-FREE_HAND_CHECK_TRUCK_URBAN_

DISPERSAL ACCIDENT INPUT

$\begin{array}{cc}\begin{array}{c}\text { AREADA } \\ \text { (M SQ) }\end{array} & \begin{array}{c}\text { DILUTION } \\ \text { FACTOR }\end{array} \\ 4.590 E+02 & 3.420 E-03 \\ 1.530 E+03 & 1.720 E-03 \\ 3.940 E+03 & 8.580 E-04 \\ 1.250 E+04 & 3.420 E-04 \\ 3.040 E+04 & 1.720 E-04 \\ 6.850 E+04 & 8.580 E-05 \\ 1.760 E+05 & 3.420 E-05 \\ 4.450 E+05 & 1.720 E-05 \\ 8.590 E+05 & 8.580 E-06 \\ 2.550 E+06 & 3.420 E-06 \\ 4.450 E+06 & 1.720 E-06 \\ 1.030 E+07 & 8.580 E-07 \\ 2.160 E+07 & 3.420 E-07 \\ 5.520 E+07 & 1.720 E-07 \\ 1.770 E+08 & 8.580 E-08 \\ 4.890 E+08 & 5.420 E-08 \\ 8.120 E+08 & 4.300 E-08 \\ 1.350 E+09 & 3.420 E-08\end{array}$

* DILUTION FACTOR UNITS ARE (CI-SEC/M**3/CI-RELEASED)

NON-DISPERSAL ACCIDENT INPUT

$\begin{array}{lcl} & \text { RADIST(M) } & \\ \text { RURAL } & \text { SUBURBAN } & \text { URBAN } \\ 3.050 E+00 & 3.050 E+00 & 3.050 E+00 \\ 6.100 E+00 & 6.100 E+00 & 6.100 E+00 \\ 9.100 E+00 & 9.100 E+00 & 9.100 E+00 \\ 1.220 E+01 & 1.220 E+01 & 1.220 E+01 \\ 1.520 E+01 & 1.520 E+01 & 1.520 E+01 \\ 3.050 E+01 & 3.050 E+01 & 3.050 E+01 \\ 6.100 E+01 & 6.100 E+01 & 6.100 E+01 \\ 9.140 E+01 & 9.140 E+01 & 9.140 E+01 \\ 1.524 E+02 & 1.524 E+02 & 1.524 E+02 \\ 3.050 E+02 & 3.050 E+02 & 3.050 E+02\end{array}$

BUILDING DOSE FACTOR

FRACTION OF LAND UNDER CULTIVATION

CONTAMINATION CLEAN UP LEVEL (UCI/M**2)

BREATHING RATE ( $\mu^{\star \star} 3 /$ SEC $)$
$=8.600 \mathrm{E}-03$

$=4.400 \mathrm{E}-01$

$=1.000 \mathrm{E}+09$

$=3.300 \mathrm{E}-04$ 
RUN DATE: [ 16-APR-95 AT 15:54:34 ]

_INCIDENT-FREE_HAND_CHECK_TRUCK_URBAN_

\author{
REGULATORY CHECKS
}

MODE 1 HAS BEEN REDESIGNATED AS EXCLUSIVE USE

FOR THE SHIPMENT OF

SNF BY MODE 1

THE DOSE RATE AT 2 METERS COULD EXCEED $10 \mathrm{MR} / H R$

PPS ${ }^{\star}$ TI HAS BEEN RESET TO EQUAL 14.00

FOR THE SHIPMENT OF

SNF BY MODE 1

THE DOSE RATE IN THE CREW COMPARTMENT COULD EXCEED 2 MREM/HR

THE DOSE RATE HAS BEEN RESET FROM 9.11 TO 2 FOR CREW CALCULATIONS 
_INCIDENT-FREE_HAND_CHECK_TRUCK_URBAN_

INCIDENT-FREE SUMMARY

INCIDENT-FREE POPULATION EXPOSURE IN PERSON-REM

PASSENGR CREW HANDLERS OFF LINK ON LINK STOPS STORAGE TOTALS LINK $1 \quad 0.00 E+00 \quad 1.67 E-04 \quad 0.00 E+00 \quad 1.08 E-04 \quad 1.50 E-04 \quad 1.20 E-04 \quad 0.00 E+00 \quad 5.44 E-04$

TOTALS: $0.00 E+00 \quad 1.67 E-04 \quad 0.00 E+00 \quad 1.08 E-04 \quad 1.50 E-04 \quad 1.20 E-04 \quad 0.00 E+00 \quad 5.44 E-04$

MAXIMUM INDIVIDUAL IN-TRANSIT DOSE

LINK $13.34 \mathrm{E}-07$ REM 
_INCIDENT-FREE_HAND_CHECK_TRUCK_URBAN_

INCIDENT-FREE IMPORTANCE ANALYSIS SUMMARY FOR LINK 1

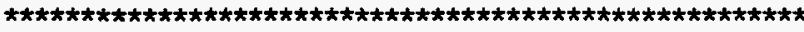

INDEX DESCRIPTION OF PARAMETER

IMPORTANCE

1 NUMBER OF SHIPMENTS

DISTANCE TRAVELED

FRACTION OF TRAVEL - URBAN

$5.444 E-06$

PACKAGES PER SHIPMENT

$5.444 E-06$

4.241E-06

$K$ ZERO

DOSE RATE (TRANSPORT INDEX)

$3.775 \mathrm{E}-06$

$3.775 \mathrm{E}-06$

$3.775 E-06$

NUMBER OF CREW MEMBERS

NUMBER OF PEOPLE PER VEHICLE

$1.669 \mathrm{E}-06$

TRAFFIC COUNT - URBAN

$1.495 E-06$

STOP TIME

$1.495 E-06$

PERSONS EXPOSED WHILE STOPPED

$1.203 E-06$

1.203E-06

FRACTION OF TRAVEL ON CITY STREETS 1.179E-06

POPULATION DENSITY - URBAN

$1.077 \mathrm{E}-06$

FRACTION OF RUSH HOUR TRAVEL

$7.339 E-07$

NUMBER OF HANDLINGS

$0.000 E+00$

EXPOSURE TIME FOR HANDLERS

$0.000 E+00$

PERSONS EXPOSED PER HANDLING

$0.000 E+00$

NUMBER OF FLIGHT ATTENDANTS

$0.000 E+00$

TRAFFIC COUNT - SUBURBAN

$0.000 E+00$

TRAFFIC COUNT - RURAL

$0.000 E+00$

FRACTION OF TRAVEL ON FREEWAYS $\quad 0.000 E+00$

HANDLER EXPOSURE DISTANCE

OISTANCE FROM SOURCE TO CREW

$0.000 E+00$

$0.000 E+00$

STORAGE EXPOSURE DISTANCE

$0.000 E+00$

NUMBER OF PERSONS EXPOSED DURING STORAGE $0.000 E+00$

FRACTION OF TRAVEL - SUBURBAN

$0.000 E+00$

RATIO OF PEDESTRIAN DENSITY (RPD) $0.000 E+00$

POPULATION DENSITY - RURAL.

$0.000 E+00$

FRACTION OF TRAVEL - RURAL

$0.000 E+00$
$0.000 E+00$

POPULATION DENSITY - SUBURBAN $0.000 E+00$

VELOCITY - SUBURBAN

$0.000 \mathrm{E}+00$

SUBURBAN SHIELDING FACTOR (RS) $\cdot 0.000 E+00$

STORAGE TIME PER SHIPMENT

$0.000 E+00$

URBAN SHIELDING FACTOR (RU)

$0.000 E+00$

$0.000 E+00$

RURAL SHIELDING FACTOR (RR)

$-1.817 E-06$

$-2.406 \mathrm{E}-06$

EXPOSURE DISTANCE WHILE STOPPED

$-3.919 E-06$

THE IMPORTANCE VALUE ESTIMATES THE PERSON-REM INFLUENCE OF A ONE PERCENT INCREASE IN THE PARAMETER

TOTAL EXPOSED POPULATION: INCIDENT-FREE

TRUCK RURAL

TRUCK SUBURBAN

TRUCK URBAN

$0.00 E+00$ PERSONS

6.18E+03 PERSONS

TOTAL

$6.18 E+03$ PERSONS

TOTAL EXPOSED POPULATION: ACCIDENT

(PERSONS UNDER PLUME FOOTPRINT FOR A SINGLE ACCIDENT)

TRUCK RURAL

TRUCK SUBURBAN

TRUCK URBAN
$0.00 E+00$ PERSONS

$0.00 E+00$ PERSONS

$0.00 E+00$ PERSONS

EOI

END OF RUN 


\section{A.4 RURFR.OUT \\ UNIT RISK FACTORS FOR RAIL IN RURAL POPULATION ZONE}




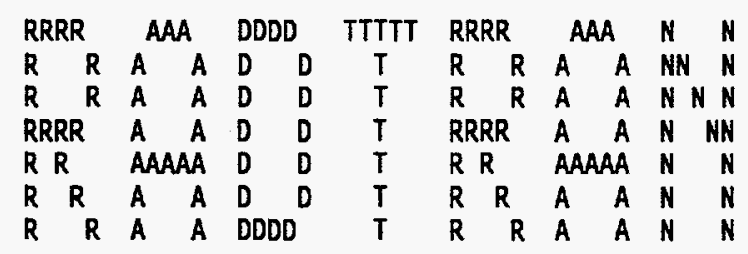

RADTRAN 4.0.17 VERSION DATE: NOVEMBER 8, 1994

MODE DESCRIPTIONS

$\begin{array}{cll}\text { NUMBER } & \text { NANE } & \text { CHARACTERIZATION } \\ 1 & \text { TRUCK } & \text { LONG HAUL VEHICLE } \\ 2 & \text { RAIL } & \text { COMMERCIAL TRAIN } \\ 3 & \text { BARGE } & \text { INLAND VESSEL } \\ 4 & \text { SHIP } & \text { OPEN SEA VESSEL } \\ 5 & \text { CARGO AIR } & \text { CARGO AIRCRAFT } \\ 6 & \text { PASS AIR } & \text { PASSENGER AIRCRAFT } \\ 7 & \text { P-VAN } & \text { PASSENGER VAN } \\ 8 & \text { CVAN-T } & \text { COMMERCIAL VAN } \\ 9 & \text { CVAN-R } & \text { COMMERCIAL VAN } \\ 10 & \text { CVAN-CA } & \text { COMHERCIAL VAN }\end{array}$


ECHO CHECK

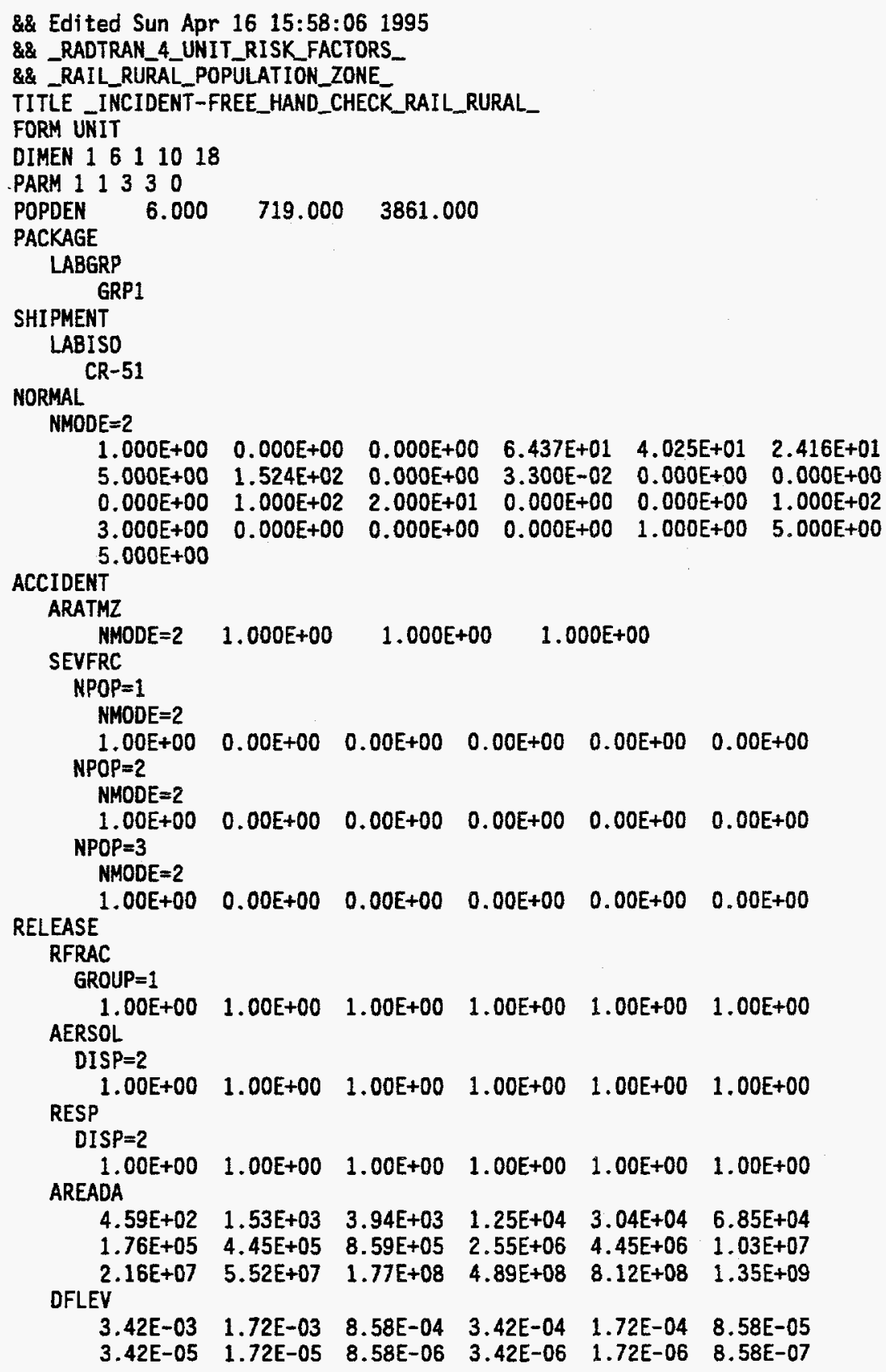


_INCIDENT-FREE_HAND_CHECK_RAIL_RURAL_

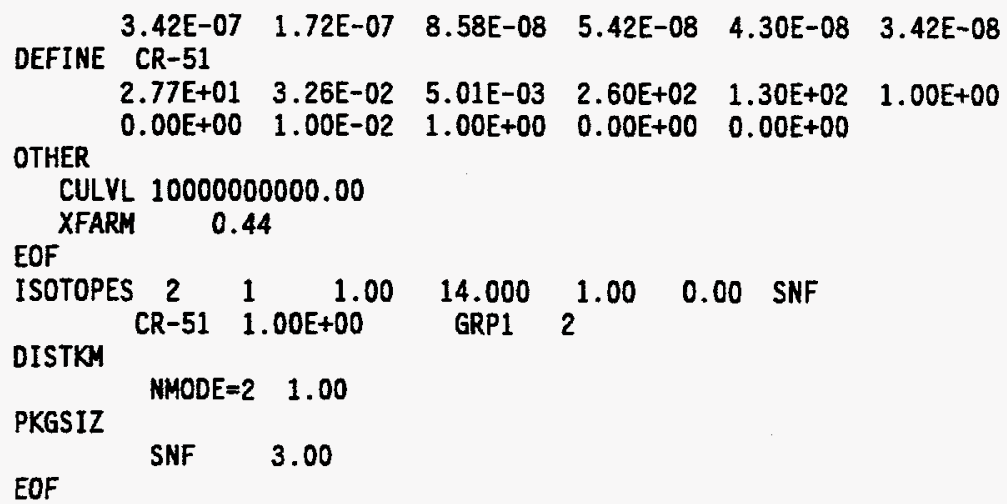




\begin{tabular}{lc}
\multicolumn{1}{c}{ ZONE } & POPULATION DENSITY \\
& (PERSONS PER SQ KM) \\
RURAL & 6. \\
SUBURBAN & 719. \\
URBAN & 3861.
\end{tabular}

PACKAGE CHARACTERISTICS

$\begin{array}{cccc}\text { FOR } & \text { DIMENSION } & \text { EFFECTIVE } & \text { K(0) } \\ \text { MATERIAL } & \text { (METERS) } & \text { DIMENSION } & \text { METERS SQ. } \\ \text { SNF } & 3.000 E+00 & 3.000 E+00 & 6.250 E+00\end{array}$

PACKAGE HANDLING THRESHOLDS (METERS)

PKESZ1 $=5.000 E-01$

PKGSZ2 $=1.000 E+00$

PACKAGES . LE. PKGSZI ARE HAND CARRIED

PACKAGES .GT. PKGSZ1 AND . LE. PKGSZ2 ARE HANDLED BY SMALL EQUIPMENT

PACKAGES .GT. PKGSZ2 ARE HANDLED BY HEAVY EQUIPMENT

\begin{tabular}{rrr} 
& \multicolumn{3}{c}{ MATERIAL CHARACTERISTICS } \\
& & \\
MATERIAL & FRACTION & FRACTION \\
SNF & OF GAMMA & OF NEUTRON \\
& $1.000 E+00$ & $0.000 E+00$
\end{tabular}


RUN DATE: [ 16-APR-95 AT 15:58:32 ]

PAGE 5

_INCIDENT-FREE_HAND_CHECK_RAIL_RURAL_

MODE CHARACTERISTICS

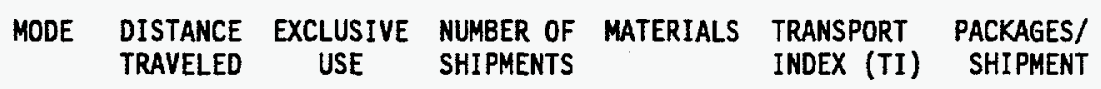

RAIL $1.00 E+00$ NO $1.00 E+00 \quad$ SNF $1.40 \mathrm{E}+01 \quad 1.00 \mathrm{E}+00$

BUILDING SHIELDING OPTION $=3$

(1=TOTAL SHIELDING, 2=PARTIAL SHIELDING, 3=NO SHIELDING)

$R P D=6.000 E+00$

(RATIO OF PEDESTRIAN DENSITY (PEDESTRIAN/KM SQ OF SIDEWALK)

TO POPULATION DENSITY (PEOPLE/KM SQ IN URBAN AREAS)

$R R=1.000 E+00$

(TRANSMISSION FACTOR FOR RURAL AREAS)

RS $=1.000 E+00$

(TRANSMISSION FACTOR FOR SUBURBAN AREAS)

$R U=1.000 E+00$

(TRANSMISSION FACTOR FOR URBAN AREAS) 
NO

1 FRACTION OF TRAVEL IN RURAL POPULATION ZONE

2 FRACTION OF TRAVEL IN SUBURBAN POPULATION ZONE

3 FRACTION OF TRAVEL IN URBAN POPULATION ZONE

4 VELOCITY IN RURAL POPULATION ZONE (KILOMETERS/HOUR)

5 VELOCITY IN SUBURBAN POP. ZONE 4.025E+D1 (KILOMETERS/HOUR)

6 VELOCITY IN URBAN POPULATION 2.416E+01 ZONE (KILOMETERS/HOUR)

7 NUMBER OF CREMMEN

$5.000 E+00$

8 DISTANCE FROM SOURCE TO CREW (METERS)

9 NUMBER OF HANDLINGS

$1.524 E+02$

$0.000 E+00$

10 STOP TIME PER KM (HR/KM)

$3.300 E-02$

11 MINIMUM STOP TIME PER TRIP (HR)

12 ZERO STOP TIME PER TRIP (HR)

$0.000 E+00$

$0.000 E+00$

13 MINIMUM NUMBER OF RAIL CLASSIF $0.000 E+00$ ICATIONS/INSPECTIONS

14 PERSONS EXPOSED WHILE STOPPED $1.000 E+02$

15 AVERAGE EXPOSURE DISTANCE WHILE STOPPED (METERS)

16 STORAGE TIME PER SHIPHENT (HR)

17 NUMBER OF EXPOSED PERSONS DURING STORAGE

18 AVERAGE EXPOSURE DISTANCE WHILE IN STORAGE (METERS)

19 NUMBER OF PEOPLE PER VEHICLE ON LINK

20 FRACTION OF URBAN TRAVEL DURING RUSH HOUR TRAFFIC

21 FRACTION OF URBAN TRAVEL ON CITY STREETS

22 FRACTION OF RURAL-SUBURBAN TRAVEL ON FREEWAYS

23 *TRAFFIC COUNT PASSING A SPECIFIC POINT-RURAL ZONE

24 tTRAFFIC COUNT PASSING A SPECIFIC POINT-SUBURBAN ZONE

25 *TRAFFIC COUNT PASSING A SPECIFIC POINT-URBAN ZONE

* (ONE WAY VEHICLES/HR)
2.000E+01

$0.000 E+00$

$0.000 E+00$

$1.000 E+02$

$3.000 E+00$

$0.000 E+00$

$0.000 E+00$

$0.000 E+00$

$1.000 E+00$

$5.000 E+00$

$5.000 E+00$ 
RUN DATE: [ 16-APR-95 AT 15:58:32]

_INCIDENT-FREE_HAND_CHECK_RAIL_RURAL_

ISOTOPE RELATED DATA

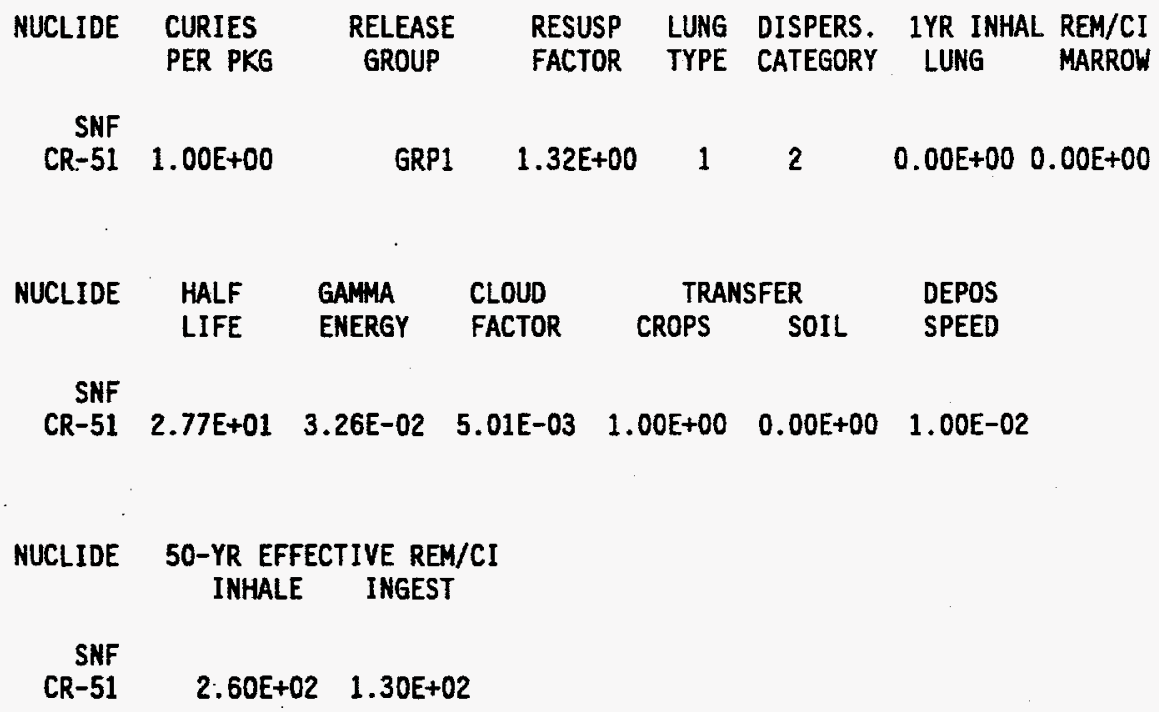


_INCIDENT-FREE_HAND_CHECK_RAIL_RURAL_

RELEASE RELATED DATA

ACCIDENT RATES (PER KM)

MODE RURAL SUBURBAN URBAN

RAIL $\quad 1.000 E+00 \quad 1.000 E+00 \quad 1.000 E+00$

\section{RELEASE FRACTIONS}

GROUP SEVER: 1 SEVER: 2 SEVER: 3 SEVER: 4 SEVER: 5 SEVER: 6

$1 \quad 1.00 E+00 \quad 1.00 E+00 \quad 1.00 E+00 \quad 1.00 E+00 \quad 1.00 E+00 \quad 1.00 E+00$

\section{ACCIDENT SEVERITY FRACTIONS}

FOR RAIL

$\begin{array}{cllllll}\text { ZONE } & \text { SEVER: } 1 & \text { SEVER: } 2 & \text { SEVER: } 3 & \text { SEVER: } 4 & \text { SEVER: } 5 & \text { SEVER: } 6 \\ 1 & 1.00 E+00 & 0.00 E+00 & 0.00 E+00 & 0.00 E+00 & 0.00 E+00 & 0.00 E+00 \\ 2 & 1.00 E+00 & 0.00 E+00 & 0.00 E+00 & 0.00 E+00 & 0.00 E+00 & 0.00 E+00 \\ 3 & 1.00 E+00 & 0.00 E+00 & 0.00 E+00 & 0.00 E+00 & 0.00 E+00 & 0.00 E+00\end{array}$


_INCIDENT-FREE_HAND_CHECK_RAIL_RURAL_

AEROSOLIZED FRACTION OF RELEASED MATERIAL

$\begin{array}{cllllll}\text { DISP CAT } & \text { SEVER: } 1 & \text { SEVER: } 2 & \text { SEVER: } 3 & \text { SEVER: } 4 & \text { SEVER: } 5 & \text { SEVER: } 6 \\ 1 & 0.00 E+00 & 0.00 E+00 & 0.00 E+00 & 0.00 E+00 & 0.00 E+00 & 0.00 E+00 \\ 2 & 1.00 E+00 & 1.00 E+00 & 1.00 E+00 & 1.00 E+00 & 1.00 E+00 & 1.00 E+00 \\ 3 & 1.00 E-02 & 1.00 E-02 & 1.00 E-02 & 1.00 E-02 & 1.00 E-02 & 1.00 E-02 \\ 4 & 5.00 E-02 & 5.00 E-02 & 5.00 E-02 & 5.00 E-02 & 5.00 E-02 & 5.00 E-02 \\ 5 & 1.00 E-01 & 1.00 E-01 & 1.00 E-01 & 1.00 E-01 & 1.00 E-01 & 1.00 E-01 \\ 6 & 1.00 E+00 & 1.00 E+00 & 1.00 E+00 & 1.00 E+00 & 1.00 E+00 & 1.00 E+00 \\ 7 & 1.00 E+00 & 1.00 E+00 & 1.00 E+00 & 1.00 E+00 & 1.00 E+00 & 1.00 E+00 \\ 8 & 1.00 E+00 & 1.00 E+00 & 1.00 E+00 & 1.00 E+00 & 1.00 E+00 & 1.00 E+00 \\ 9 & 1.00 E+00 & 1.00 E+00 & 1.00 E+00 & 1.00 E+00 & 1.00 E+00 & 1.00 E+00 \\ 10 & 1.00 E+00 & 1.00 E+00 & 1.00 E+00 & 1.00 E+00 & 1.00 E+00 & 1.00 E+00 \\ 11 & 1.00 E+00 & 1.00 E+00 & 1.00 E+00 & 1.00 E+00 & 1.00 E+00 & 1.00 E+00\end{array}$

FRACTION OF AEROSOLS BELOW 10 MICRONS AED

$\begin{array}{cllllll}\text { DISP CAT } & \text { SEVER: } 1 & \text { SEVER: } 2 & \text { SEVER: } 3 & \text { SEVER: } 4 & \text { SEVER: } 5 & \text { SEVER: } 6 \\ 1 & 0.00 E+00 & 0.00 E+00 & 0.00 E+00 & 0.00 E+00 & 0.00 E+00 & 0.00 E+00 \\ 2 & 1.00 E+00 & 1.00 E+00 & 1.00 E+00 & 1.00 E+00 & 1.00 E+00 & 1.00 E+00 \\ 3 & 5.00 E-02 & 5.00 E-02 & 5.00 E-02 & 5.00 E-02 & 5.00 E-02 & 5.00 E-02 \\ 4 & 5.00 E-02 & 5.00 E-02 & 5.00 E-02 & 5.00 E-02 & 5.00 E-02 & 5.00 E-02 \\ 5 & 5.00 E-02 & 5.00 E-02 & 5.00 E-02 & 5.00 E-02 & 5.00 E-02 & 5.00 E-02 \\ 6 & 5.00 E-02 & 5.00 E-02 & 5.00 E-02 & 5.00 E-02 & 5.00 E-02 & 5.00 E-02 \\ 7 & 1.00 E+00 & 1.00 E+00 & 1.00 E+00 & 1.00 E+00 & 1.00 E+00 & 1.00 E+00 \\ 8 & 1.00 E+00 & 1.00 E+00 & 1.00 E+00 & 1.00 E+00 & 1.00 E+00 & 1.00 E+00 \\ 9 & 1.00 E+00 & 1.00 E+00 & 1.00 E+00 & 1.00 E+00 & 1.00 E+00 & 1.00 E+00 \\ 10 & 1.00 E+00 & 1.00 E+00 & 1.00 E+00 & 1.00 E+00 & 1.00 E+00 & 1.00 E+00 \\ 11 & 1.00 E+00 & 1.00 E+00 & 1.00 E+00 & 1.00 E+00 & 1.00 E+00 & 1.00 E+00\end{array}$


RUN DATE: [ 16-APR-95 AT 15:58:32 ]

_INCIOENT-FREE_HAND_CHECK_RAIL_RURAL_.

COST RELATED DATA

EMERGENCY RESPONSE COST

SEVER: 1 SEVER: 2 SEVER: 3 SEVER: 4 SEVER: 5 SEVER: 6 $0.00 E+00 \quad 0.00 E+00 \quad 0.00 E+00 \quad 0.00 E+00 \quad 0.00 E+00 \quad 0.00 E+00$

ON-SCENE COSTS

(RF=RELEASE FRACTION)

RF $=0$.

0 .

$0 .<R F<=.01$

0. $.01<R F<=0.1$

$.1<R F<=1$.

0. 
RUN DATE: [ 16-APR-95 AT 15:58:32 ]

_INCIDENT-FREE_HAND_CHECK_RAIL_RURAL_

HEALTH RELATED DATA

EARLY FATALITY PROBABILITIES

$\begin{array}{rrrrr}\text { DOSE (REM) } & \text { LUNG-1 } & \text { LUNG-2 } & \text { LUNG-3 } & \text { MARROW } \\ 100000.000 & 1.000 E+00 & 1.000 E+00 & 1.000 E+00 & 1.000 E+00 \\ 80000.000 & 1.000 E+00 & 8.500 E-01 & 8.000 E-01 & 1.000 E+00 \\ 70000.000 & 1.000 E+00 & 8.000 E-01 & 5.000 E-11 & 1.000 E+00 \\ 40000.000 & 1.000 E+00 & 7.00 E-01 & 0.000 E+00 & 1.000 E+00 \\ 30000.000 & 1.000 E+00 & 5.000 E-01 & 0.000 E+00 & 1.000 E+00 \\ 25000.000 & 1.000 E+00 & 2.000 E-01 & 0.000 E+00 & 1.000 E+00 \\ 20000.000 & 1.000 E+00 & 8.000 E-02 & 0.000 E+00 & 1.000 E+00 \\ 10000.000 & 6.000 E-01 & 0.000 E+00 & 0.000 E+00 & 1.000 E+00 \\ 8000.000 & 1.000 E-01 & 0.000 E+00 & 0.000 E+00 & 1.000 E+00 \\ 6000.000 & 6.000 E-02 & 0.000 E+00 & 0.000 E+00 & 1.000 E+00 \\ 4000.000 & 3.000 E-02 & 0.000 E+00 & 0.000 E+00 & 1.000 E+00 \\ 3000.000 & 0.000 E+00 & 0.000 E+00 & 0.000 E+00 & 1.000 E+00 \\ 2000.000 & 0.000 E+00 & 0.00 E+00 & 0.000 E+00 & 1.000 E+00 \\ 1000.000 & 0.000 E+00 & 0.000 E+00 & 0.000 E+00 & 1.000 E+00 \\ 800.000 & 0.000 E+00 & 0.000 E+00 & 0.000 E+00 & 9.960 E-01 \\ 700.000 & 0.000 E+00 & 0.000 E+00 & 0.000 E+00 & 9.000 E-01 \\ 600.000 & 0.000 E+00 & 0.000 E+00 & 0.000 E+00 & 4.000 E-01 \\ 500.000 & 0.000 E+00 & 0.000 E+00 & 0.000 E+00 & 5.000 E-02 \\ 400.000 & 0.000 E+00 & 0.000 E+00 & 0.000 E+00 & 0.000 E+00 \\ 300.000 & 0.000 E+00 & 0.00 E E+00 & 0.000 E+00 & 0.000 E+00 \\ 100.000 & 0.000 E+00 & 0.000 E+00 & 0.000 E+00 & 0.000 E+00 \\ 75.000 & 0.000 E+00 & 0.000 E+00 & 0.000 E+00 & 0.000 E+00 \\ 50.000 & 0.000 E+00 & 0.000 E+00 & 0.000 E+00 & 0.000 E+00 \\ 30.000 & 0.000 E+00 & 0.000 E+00 & 0.000 E+00 & 0.000 E+00 \\ 15.000 & 0.000 E+00 & 0.000 E+00 & 0.000 E+00 & 0.000 E+00 \\ 5.000 & 0.000 E+00 & 0.000 E+00 & 0.000 E+00 & 0.000 E+00 \\ 1.000 & 0.000 E+00 & 0.000 E+00 & 0.000 E+00 & 0.000 E+00 \\ 0.100 & 0.000 E+00 & 0.000 E+00 & 0.000 E+00 & 0.000 E+00 \\ 0.010 & 0.000 E+00 & 0.000 E+00 & 0.000 E+00 & 0.000 E+00 \\ 0.010 & 0.000 E+00 & 0.000 E+00 & 0.000 E+00 & 0.000 E+00\end{array}$


_INCIDENT-FREE_HAND_CHECK_RAIL_RURAL_

DISPERSAL ACCIDENT INPUT

$\begin{array}{cc}\begin{array}{c}\text { AREADA } \\ \text { (M SQ) }\end{array} & \begin{array}{c}\text { DILUTION } \\ \text { FACTOR }\end{array} \\ 4.590 E+02 & 3.420 E-03 \\ 1.530 E+03 & 1.720 E-03 \\ 3.940 E+03 & 8.580 E-04 \\ 1.250 E+04 & 3.420 E-04 \\ 3.040 E+04 & 1.720 E-04 \\ 6.850 E+04 & 8.580 E-05 \\ 1.760 E+05 & 3.420 E-05 \\ 4.450 E+05 & 1.720 E-05 \\ 8.590 E+05 & 8.580 E-06 \\ 2.550 E+06 & 3.420 E-06 \\ 4.450 E+06 & 1.720 E-06 \\ 1.030 E+07 & 8.580 E-07 \\ 2.160 E+07 & 3.420 E-07 \\ 5.520 E+07 & 1.720 E-07 \\ 1.770 E+08 & 8.580 E-08 \\ 4.890 E+08 & 5.420 E-08 \\ 8.120 E+08 & 4.300 E-08 \\ 1.350 E+09 & 3.420 E-08\end{array}$

* DILUTION FACTOR UNITS ARE (CI-SEC/M**3/CI-RELEASED)

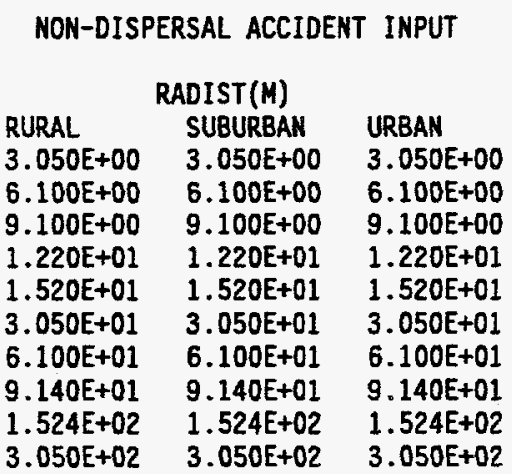

BUILDING DOSE FACTOR

FRACTION OF LAND UNDER CULTIVATION

CONTAMINATION CLEAN UP LEVEL (UCI/M**2)

BREATHING RATE ( $M^{\star \star} 3 /$ SEC)
$=8.600 \mathrm{E}-03$

$=4.400 \mathrm{E}-01$

$=1.000 E+09$

$=3.300 \mathrm{E}-04$ 
RUN DATE: [ 16-APR-95 AT 15:58:32 ]

_INCIDENT-FREE_HAND_CHECK_RAIL_RURAL_

REGULATORY CHECKS

MODE 2 HAS BEEN REDESIGNATED AS EXCLUSIVE USE

FOR THE SHIPMENT OF SNF BY MODE 2

THE DOSE RATE AT 2 METERS COULD EXCEED $10 \mathrm{MR} / \mathrm{HR}$

PPS*TI HAS BEEN RESET TO EQUAL 14.00 
_INCIDENT-FREE_HAND_CHECK_RAIL_RURAL_

INCIDENT-FREE SUMMARY

INCIDENT-FREE POPULATION EXPOSURE IN PERSON-REM

PASSENGR CREW HANDLERS OFF LINK ON LINK STOPS STORAGE TOTALS LINK $10.00 E+00 \quad 1.01 E-05 \quad 0.00 E+00 \quad 1.70 E-07 \quad 6.62 E-08 \quad 4.78 E-06 \quad 0.00 E+00 \quad 1.51 E-05$

TOTALS: $0.00 E+00 \quad 1.01 E-05 \quad 0.00 E+00 \quad 1.70 E-07 \quad 6.62 E-08 \quad 4.78 E-06 \quad 0.00 E+00 \quad 1.51 E-05$

MAXIMUM INDIVIDUAL IN-TRANSIT DOSE

LINK $1 \quad 3.34 \mathrm{E}-07$ REM 
_INCIDENT-FREE_HAND_CHECK_RAIL_RURAL_

INCIDENT-FREE IMPORTANCE ANALYSIS SUMMARY FOR LINK 1

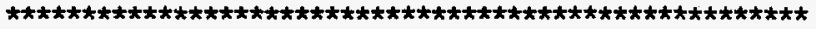

INDEX

DESCRIPTION OF PARAMETER

DOSE RATE (TRANSPORT INDEX)

NUMBER OF SHIPMENTS

$K$ ZERO

PACKAGES PER SHIPMENT

DISTANCE TRAVELED

PERSONS EXPOSED WHILE STOPPED

STOP TIME

FRACTION OF TRAVEL - RURAL

POPULATION DENSITY - RURAL

TRAFFIC COUNT - RURAL

NUMBER OF PEOPLE PER VEHICLE

NUMBER OF HANDLINGS

DISTANCE FROM SOURCE TO CREW

NUMBER OF CREW MEMBERS

EXPOSURE TIME FOR HANDLERS

PERSONS EXPOSED PER HANDLING

HANDLER EXPOSURE DISTANCE

NUMBER OF FLIGHT ATTENDANTS

TRAFFIC COUNT - URBAN

TRAFFIC COUNT - SUBURBAN

FRACTION OF RUSH HOUR TRAVEL

FRACTION OF TRAVEL ON FREEWAYS

STORAGE EXPOSURE DISTANCE

NUMBER OF PERSONS EXPOSED DURING STORAGE

SUBURBAN SHIELDING FACTOR (RS)

VELOCITY - SUBURBAK

POPULATION DENSITY - SUBURBAN

FRACTION OF TRAVEL - SUBURBAN

RATIO OF PEDESTRIAN DENSITY (RPD)

FRACTION OF TRAVEL - URBAN

POPULATION DENSITY - URBAN

STORAGE TIME PER SHIPMENT

URBAN SHIELDIMG FACTOR (RU)

FRACTION OF TRAVEL ON CITY STREETS

VELOCITY - URBAN

RURAL SHIELDING FACTOR (RR)

VELOCITY - RURAL

EXPOSURE DISTANCE WHILE STOPPED
IMPORTANCE

1.509E-07

1.509E-07

1. $509 \mathrm{E}-07$

1.509E-07

$5.011 \mathrm{E}-08$

4.775E-08

4.775E-08

2.358E-09

$1.696 \mathrm{E}-09$

6. $620 \mathrm{E}-10$

6. $620 \mathrm{E}-10$

$0.000 E+00$

$0.000 E+00$

$0.000 E+00$

$0.000 E+00$

$0.000 E+00$

$0.000 E+00$

$0.000 E+00$

$0.000 E+00$

$0.000 E+00$

$0.000 E+00$

$0.000 E+00$

$0.000 E+00$

$0.000 E+00$

$0.000 E+00$

$0.000 E+00$

$0.000 E+00$

$0.000 E+00$

$0.000 E+00$

$0.000 E+00$

$0.000 E+00$

$0.000 E+00$

$0.000 E+00$

$0.000 E+00$

$0.000 E+00$

$0.000 E+00$

$-3.020 E-09$

$-9.550 E-08$

THE IMPORTANCE VALUE ESTIMATES THE PERSON-REM INFLUENCE OF A ONE PERCENT INCREASE IN THE PARAMETER

TOTAL EXPOSED POPULATION: INCIDENT-FREE

RAIL RURAL

RAIL SUBURBAN

RAIL URBAN

9.60E+DO PERSONS

$0.00 E+00$ PERSONS

$0.00 E+00$ PERSONS

TOTAL 9.6OE+OO PERSONS

TOTAL EXPOSED POPULATION: ACCIDENT

(PERSONS UNDER PLUHE FOOTPRINT FOR A SINGLE ACCIDENT)

EOI
RAIL RURAL
- RAIL SUBURBAN
$0.00 E+00$ PERSONS
$0.00 E+00$ PERSONS
RAIL URBAN
$0.00 E+00$ PERSONS

END OF RUN 
A-74 


\section{A.5 RURFS.OUT}

UNIT RISK FACTORS FOR RAIL IN SUBURBAN POPULATION ZONE 
A-76 


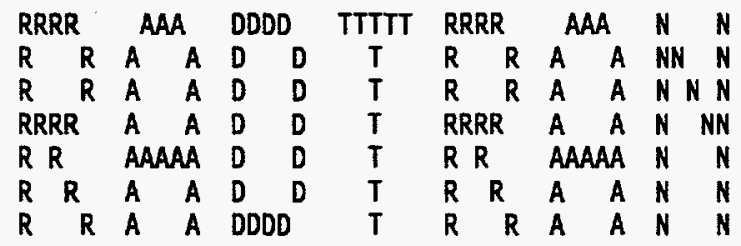

4
4
4
44444
4
4
4

RADTRAN 4.0.17 VERSION DATE: NOVEMBER 8, 1994

MODE DESCRIPTIONS

$\begin{array}{cll}\text { MUMBER } & \text { NAME } & \text { CHARACTERIZATION } \\ 1 & \text { TRUCK } & \text { LONG HAUL VEHICLE } \\ 2 & \text { RAIL } & \text { COMMERCIAL TRAIN } \\ 3 & \text { BARGE } & \text { INLAND VESSEL } \\ 4 & \text { SHIP } & \text { OPEN SEA VESSEL } \\ 5 & \text { CARGO AIR } & \text { CARGO AIRCRAFT } \\ 6 & \text { PASS AIR } & \text { PASSENGER AIRCRAFT } \\ 7 & \text { P-VAN } & \text { PASSENGER VAN } \\ 8 & \text { CVAN-T } & \text { COMMERCIAL VAN } \\ 9 & \text { CVAN-R } & \text { COMMERCIAL VAN } \\ 10 & \text { CVAN-CA } & \text { COMMERCIAL VAN }\end{array}$


ECHO CHECK

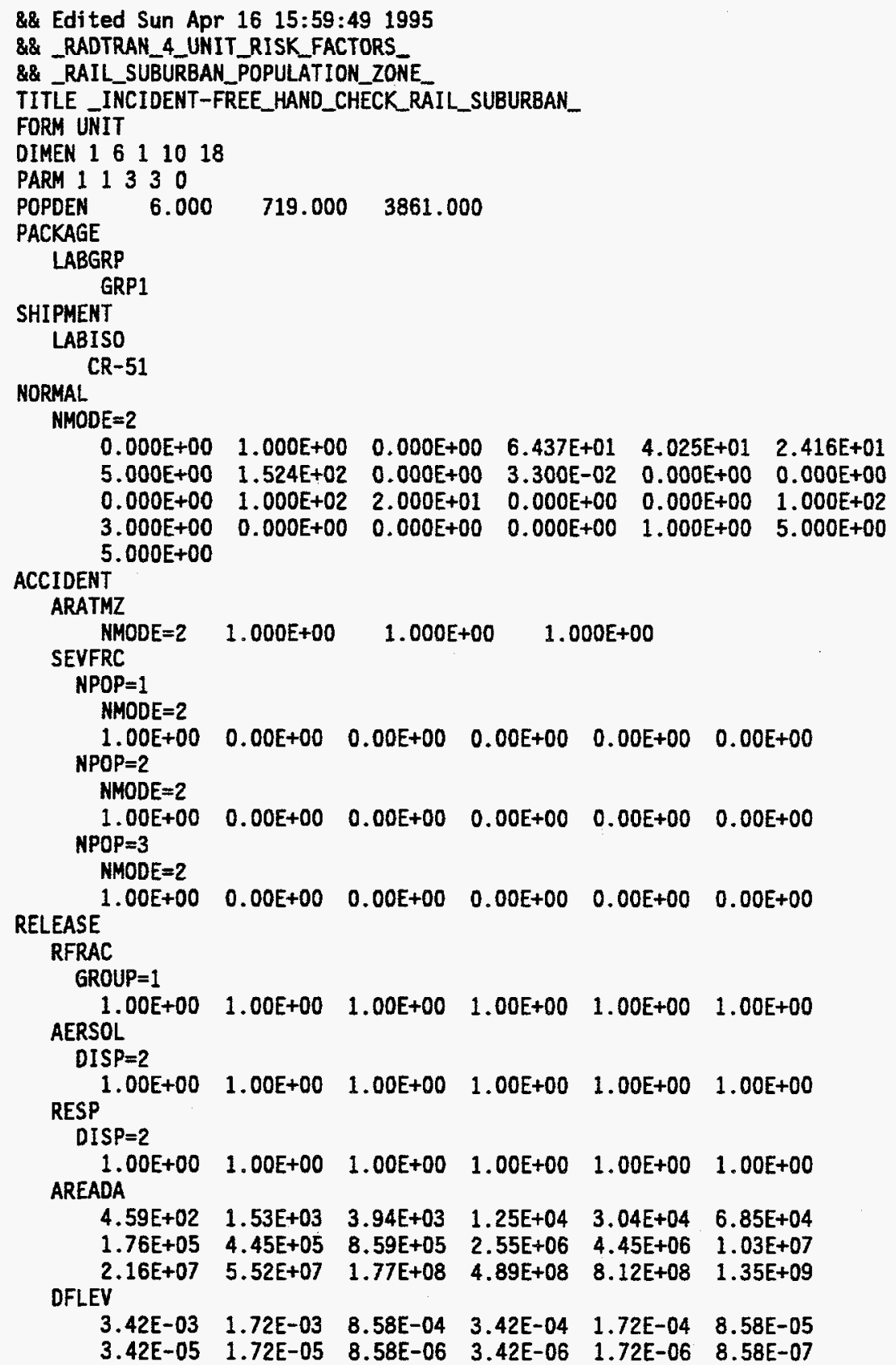


RUN DATE: [ 16-APR-95 AT 16:00:25 ]

_INCIDENT-FREE_HANO_CHECK_RAIL_SUBURBAN_

$\begin{array}{llllll}3.42 E-07 & 1.72 E-07 & 8.58 E-08 & 5.42 E-08 & 4.30 E-08 & 3.42 E-08\end{array}$

DEFINE CR-51

$2.77 E+01 \quad 3.26 E-02 \quad 5.01 E-03 \quad 2.60 E+02 \quad 1.30 E+02 \quad 1.00 E+00$

OTHER

$0.00 E+00 \quad 1.00 E-02 \quad 1.00 E+00 \quad 0.00 E+00 \quad 0.00 E+00$

\section{CULVL 10000000000.00} XF

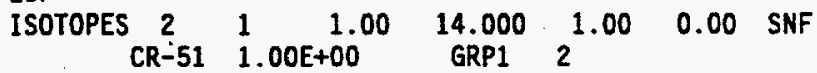

DISTKM

PKGSIZ

NMODE $=2 \quad 1.00$

EOF

SNF $\quad 3.00$


_INCIDENT-FREE_HAND_CHECK_RAIL_SUBURBAN_

\begin{tabular}{lc}
\multicolumn{1}{c}{ ZONE } & POPULATION DENSITY \\
& (PERSONS PER SQ KM) \\
RURAL & 6. \\
SUBURBAN & 719. \\
URBAN & 3861.
\end{tabular}

PACKAGE CHARACTERISTICS

$\begin{array}{rrrl}\text { FOR } & \text { DIMENSION } & \text { EFFECTIVE } & K(0) \\ \text { MATERIAL } & \text { (METERS) } & \text { DIMENSION } & \text { METERS SQ. } \\ \text { SNF } & 3.000 E+00 & 3.000 E+00 & 6.250 E+00\end{array}$

$K(0)$ IS TI TO DOSE RATE CONVERSION FACTOR

PACKAGE HANDLING THRESHOLDS (METERS)

PKGSZ1 $=5.000 \mathrm{E}-01$

PKGSZ2 $=1.000 E+00$

PACKAGES .LE. PKGSZI ARE HAND CARRIED

PACKAGES .GT. PKGSZ1 AND .LE. PKGSZ2 ARE HANDLED BY SMALL EQUIPMENT

PACKAGES .GT. PKGSZ2 ARE HANDLED BY HEAVY EQUIPMENT

MATERIAL CHARACTERISTICS

$\begin{array}{rrr} & \text { FRACTION } & \text { FRACTION } \\ \text { MATERIAL } & \text { OF GAMMA } & \text { OF NEUTRON } \\ \text { SNF } & 1.000 E+00 & 0.000 E+00\end{array}$


RUN DATE: [ 16-APR-95 AT 16:00:25 ]

_INCIDENT-FREE_HAND_CHECK_RAIL_SUBURBAN_

MODE CHARACTERISTICS

$\begin{array}{ccrrrrr}\text { MODE } & \begin{array}{c}\text { DISTANCE } \\ \text { TRAVELED }\end{array} & \begin{array}{c}\text { EXCLUSIVE } \\ \text { USE }\end{array} & \begin{array}{c}\text { NUMBER OF } \\ \text { SHIPMENTS }\end{array} & \text { MATERIALS } & \begin{array}{c}\text { TRANSPORT } \\ \text { INDEX (TI) }\end{array} & \begin{array}{c}\text { PACKAGES/ } \\ \text { SHIPMENT }\end{array} \\ \text { RAIL } & 1.00 E+00 & \text { NO } & 1.00 E+00 & & & \\ & & & & \text { SNF } & 1.40 E+01 & 1.00 E+00\end{array}$

BUILDING SHIELDING OPTION= 3

(1=TOTAL SHIELDING, 2=PARTIAL SHIELDING, 3=NO SHIELDING)

$R P D=6.000 E+00$

(RATIO OF PEDESTRIAN DENSITY (PEDESTRIAN/KM SQ OF SIDEWALK)

TO POPULATION DENSITY (PEOPLE/KM SQ IN URBAN AREAS)

$R R=1.000 E+00$

(TRANSMISSION FACTOR FOR RURAL AREAS)

RS $=1.000 E+00$

(TRANSMISSION FACTOR FOR SUBURBAN AREAS)

$\mathrm{RU}=1.000 \mathrm{E}+00$

(TRANSMISSION FACTOR FOR URBAN AREAS) 
NO

1 FRACTION OF TRAVEL IN RURAL POPULATION ZONE

2 FRACTION OF TRAVEL IN SUBURBAN POPULATION ZONE

3 FRACTION OF TRAVEL IN URBAN POPULATION ZONE

4 VELOCITY IN RURAL POPULATION ZONE (KILOMETERS/HOUR)

5 VELOCITY IN SUBURBAN POP. ZONE 4.025E+01 (KILOMETERS/HOUR)

6 VELOCITY IN URBAN POPULATION $2.416 \mathrm{E}+01$ ZONE (KILOMETERS/HOUR)

7 NUMBER OF CREWMEN $\quad 5.000 E+00$

8 DISTANCE FROM SOURCE TO CREW 1.524E+02 (METERS)

9 NUMBER OF HANDLINGS $\quad 0.000 E+00$

10 STOP TIME PER KN (HR/KM) 3.300E-02

11 MINIMUM STOP TIME PER TRIP $0.000 E+00$ (HR)

12 ZERO STOP TIME PER TRIP (HR) 0.000E+00

13 MINIMUM NUMBER OF RAIL CLASSIF $0.000 E+00$ ICATIONS/INSPECTIONS

14 PERSONS EXPOSED WHILE STOPPED $1.000 E+02$

15 AVERAGE EXPOSURE DISTANCE $2.000 E+01$ WHILE STOPPED (METERS)

16 STORAGE TIME PER SHIPMENT $0.000 E+00$ (HR)

17 NUMBER OF EXPOSED PERSONS $0.000 E+00$ DURING STORAGE

18 AVERAGE EXPOSURE DISTANCE $1.000 E+02$ WHILE IN STORAGE (METERS)

19 NUMBER OF PEOPLE PER VEHICLE 3.000E+00 ON LINK

20 FRACTION OF URBAN TRAVEL DURING RUSH HOUR TRAFFIC

21 FRACTION OF URBAN TRAVEL ON CITY STREETS

22 FRACTION OF RURAL-SUBURBAN TRAVEL ON FREEWAYS

23 *TRAFFIC COUNT PASSING A SPECIFIC POINT-RURAL ZONE

24 *TRAFFIC COUNT PASSING A SPECIFIC POINT-SUBURBAN ZONE

25 *TRAFFIC COUNT PASSING A SPECIFIC POINT-URBAN ZONE * (ONE WAY VEHICLES/HR)
DNORML INPUT

$1.000 E+00$

$0.000 E+00$

$6.437 E+01$

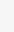


RUN DATE: [ 16-APR-95 AT 16:00:25 ]

PAGE 7

_INCIDENT-FREE_HAND_CHECK_RAIL_SUBURBAN_

ISOTOPE RELATED DATA

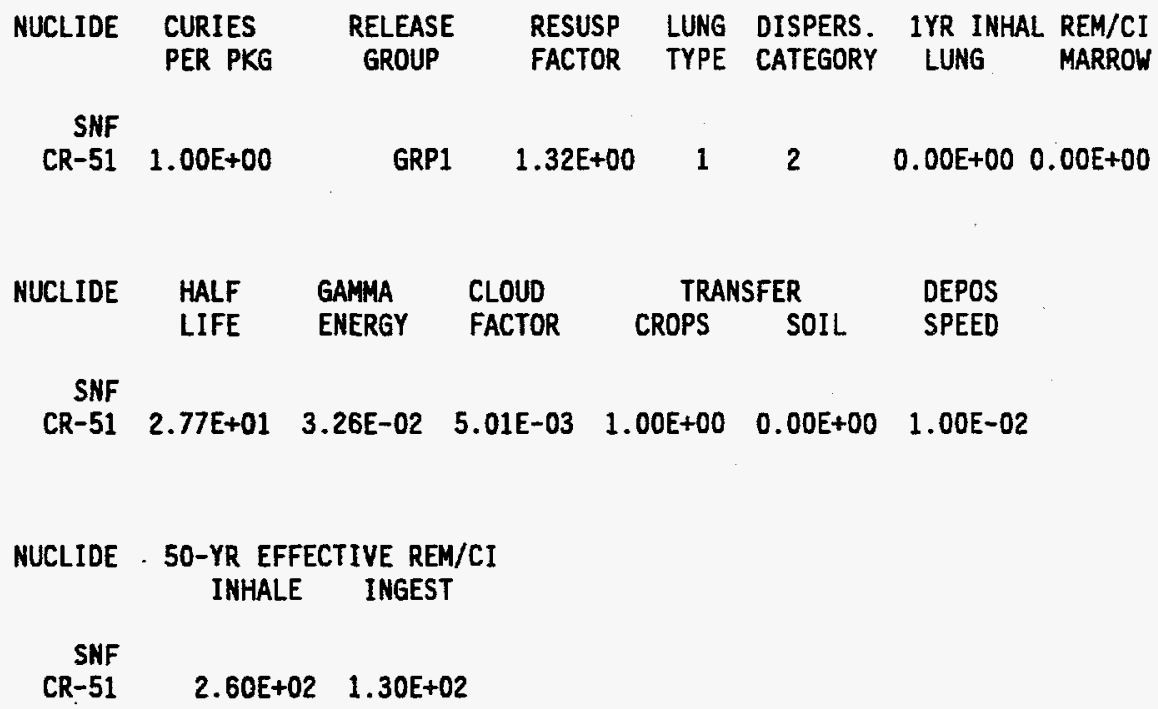

$\begin{array}{rrr}\text { SNF } \\ \text { CR-51 } & 2.60 E+02 \quad 1.30 E+02\end{array}$ 
_INCIDENT-FREE_HAND_CHECK_RAIL_SUBURBAN_

RELEASE RELATED DATA

\begin{tabular}{lrrr}
\multicolumn{4}{c}{ ACCIDENT RATES (PER KM) } \\
MODE & RURAL & SUBURBAN & URBAN \\
RAIL & $1.000 \mathrm{E}+00$ & $1.000 \mathrm{D}+00$ & $1.000 \mathrm{E}+00$
\end{tabular}

RELEASE FRACTIONS

GROUP SEVER: 1 SEVER: 2 SEVER: 3 SEVER: 4 SEVER: 5 SEVER: 6

$1 \quad 1.00 E+00 \quad 1.00 E+00 \quad 1.00 E+00 \quad 1.00 E+00 \quad 1.00 E+00 \quad 1.00 E+00$

ACCIDENT SEVERITY FRACTIONS

FOR RAIL

$\begin{array}{ccccccc}\text { ZONE } & \text { SEVER: } 1 & \text { SEVER: } 2 & \text { SEVER: } 3 & \text { SEVER: } 4 & \text { SEVER: } 5 & \text { SEVER: } 6 \\ 1 & 1.00 E+00 & 0.00 E+00 & 0.00 E+00 & 0.00 E+00 & 0.00 E+00 & 0.00 E+00 \\ 2 & 1.00 E+00 & 0.00 E+00 & 0.00 E+00 & 0.00 E+00 & 0.00 E+00 & 0.00 E+00 \\ 3 & 1.00 E+00 & 0.00 E+00 & 0.00 E+00 & 0.00 E+00 & 0.00 E+00 & 0.00 E+00\end{array}$


_INCIDENT-FREE_HAND_CHECK_RAIL_SUBURBAN_

AEROSOLIZED FRACTION OF RELEASED MATERIAL

$\begin{array}{ccccccc}\text { DISP CAT } & \text { SEVER: } 1 & \text { SEVER: } 2 & \text { SEVER: } 3 & \text { SEVER: } 4 & \text { SEVER: } 5 & \text { SEVER: } 6 \\ 1 & 0.00 E+00 & 0.00 E+00 & 0.00 E+00 & 0.00 E+00 & 0.00 E+00 & 0.00 E+00 \\ 2 & 1.00 E+00 & 1.00 E+00 & 1.00 E+00 & 1.00 E+00 & 1.00 E+00 & 1.00 E+00 \\ 3 & 1.00 E-02 & 1.00 E-02 & 1.00 E-02 & 1.00 E-02 & 1.00 E-02 & 1.00 E-02 \\ 4 & 5.00 E-02 & 5.00 E-02 & 5.00 E-02 & 5.00 E-02 & 5.00 E-02 & 5.00 E-02 \\ 5 & 1.00 E-01 & 1.00 E-01 & 1.00 E-01 & 1.00 E-01 & 1.00 E-01 & 1.00 E-01 \\ 6 & 1.00 E+00 & 1.00 E+00 & 1.00 E+00 & 1.00 E+00 & 1.00 E+00 & 1.00 E+00 \\ 7 & 1.00 E+00 & 1.00 E+00 & 1.00 E+00 & 1.00 E+00 & 1.00 E+00 & 1.00 E+00 \\ 8 & 1.00 E+00 & 1.00 E+00 & 1.00 E+00 & 1.00 E+00 & 1.00 E+00 & 1.00 E+00 \\ 9 & 1.00 E+00 & 1.00 E+00 & 1.00 E+00 & 1.00 E+00 & 1.00 E+00 & 1.00 E+00 \\ 10 & 1.00 E+00 & 1.00 E+00 & 1.00 E+00 & 1.00 E+00 & 1.00 E+00 & 1.00 E+00 \\ 11 & 1.00 E+00 & 1.00 E+00 & 1.00 E+00 & 1.00 E+00 & 1.00 E+00 & 1.00 E+00\end{array}$

FRACTION OF AEROSOLS BELOW 10 MICRONS AED

$\begin{array}{ccccccc}\text { DISP CAT } & \text { SEVER: } 1 & \text { SEVER: } 2 & \text { SEVER: } 3 & \text { SEVER: } 4 & \text { SEVER: } 5 & \text { SEVER: } 6 \\ 1 & 0.00 E+00 & 0.00 E+00 & 0.00 E+00 & 0.00 E+00 & 0.00 E+00 & 0.00 E+00 \\ 2 & 1.00 E+00 & 1.00 E+00 & 1.00 E+00 & 1.00 E+00 & 1.00 E+00 & 1.00 E+00 \\ 3 & 5.00 E-02 & 5.00 E-02 & 5.00 E-02 & 5.00 E-02 & 5.00 E-02 & 5.00 E-02 \\ 4 & 5.00 E-02 & 5.00 E-02 & 5.00 E-02 & 5.00 E-02 & 5.00 E-02 & 5.00 E-02 \\ 4 & 5.00 E-02 & 5.00 E-02 & 5.00 E-02 & 5.00 E-02 & 5.00 E-02 & 5.00 E-02 \\ 5 & 5.00 E-02 & 5.00 E-02 & 5.00 E-02 & 5.00 E-02 & 5.00 E-02 & 5.00 E-02 \\ 6 & 1.00 E+00 & 1.00 E+00 & 1.00 E+00 & 1.00 E+00 & 1.00 E+00 & 1.00 E+00 \\ 7 & 1.00 E+00 & 1.00 E+00 & 1.00 E+00 & 1.00 E+00 & 1.00 E+00 & 1.00 E+00 \\ 8 & 1.00 E+00 & 1.00 E+00 & 1.00 E+00 & 1.00 E+00 & 1.00 E+00 & 1.00 E+00 \\ 9 & 1.00 E+00 & 1.00 E+00 & 1.00 E+00 & 1.00 E+00 & 1.00 E+00 & 1.00 E+00 \\ 10 & 1.00 & 1.00 \\ 11 & 1.00 E+00 & 1.00 E+00 & 1.00 E+00 & 1.00 E+00 & 1.00 E+00 & 1.00 E+00\end{array}$


RUN DATE: [ 16-APR-95 AT 16:00:25]

_INCIDENT-FREE_HAND_CHECK_RAIL_SUBURBAN_

COST RELATED DATA

EMERGENCY RESPONSE COST

SEVER: 1 SEVER: 2 SEVER: 3 SEVER: 4 SEVER: 5 SEVER: 6

ON-SCENE COSTS

(RF=RELEASE FRACTION)

$R F=0 . \quad 0 .<R F<=.01 \quad .01<R F<=0.1 \quad .1<R F<=1$.

o.

0 . 0 .

0. 
RUN DATE: [ 16-APR-95 AT 16:00:25]

_INCIDENT-FREE_HAND_CHECK_RAIL_SUBURBAN_

HEALTH RELATED DATA

\section{EARLY FATALITY PROBABILITIES}

$\begin{array}{rrrrr}\text { DOSE(REM) } & \text { LUNG-1 } & \text { LUNG-2 } & \text { LUNG-3 } & \text { MARROW } \\ 100000.000 & 1.000 E+00 & 1.000 E+00 & 1.000 E+00 & 1.000 E+00 \\ 80000.000 & 1.000 E+00 & 8.500 E-01 & 8.000 E-01 & 1.000 E+00 \\ 70000.000 & 1.000 E+00 & 8.000 E-01 & 5.000 E-01 & 1.000 E+00 \\ 40000.000 & 1.000 E+00 & 7.000 E-01 & 0.000 E+00 & 1.000 E+00 \\ 30000.000 & 1.000 E+00 & 5.000 E-01 & 0.000 E+00 & 1.000 E+00 \\ 25000.000 & 1.000 E+00 & 2.000 E-01 & 0.000 E+00 & 1.000 E+00 \\ 20000.000 & 1.000 E+00 & 8.000 E-02 & 0.000 E+00 & 1.000 E+00 \\ 10000.000 & 6.000 E-01 & 0.000 E+00 & 0.000 E+00 & 1.000 E+00 \\ 8000.000 & 1.000 E-01 & 0.000 E+00 & 0.000 E+00 & 1.000 E+00 \\ 6000.000 & 6.000 E-02 & 0.000 E+00 & 0.000 E+00 & 1.000 E+00 \\ 4000.000 & 3.000 E-02 & 0.000 E+00 & 0.000 E+00 & 1.000 E+00 \\ 3000.000 & 0.000 E+00 & 0.000 E+00 & 0.000 E+00 & 1.000 E+00 \\ 2000.000 & 0.000 E+00 & 0.000 E+00 & 0.000 E+00 & 1.000 E+00 \\ 1000.000 & 0.000 E+00 & 0.000 E+00 & 0.000 E+00 & 1.000 E+00 \\ 800.000 & 0.000 E+00 & 0.000 E+00 & 0.000 E+00 & 9.960 E-01 \\ 700.000 & 0.000 E+00 & 0.000 E+00 & 0.000 E+00 & 9.000 E-01 \\ 600.000 & 0.000 E+00 & 0.000 E+00 & 0.000 E+00 & 4.000 E-01 \\ 500.000 & 0.000 E+00 & 0.000 E+00 & 0.000 E+00 & 5.000 E-02 \\ 400.000 & 0.000 E+00 & 0.000 E+00 & 0.000 E+00 & 0.000 E+00 \\ 300.000 & 0.000 E+00 & 0.000 E+00 & 0.000 E+00 & 0.000 E+00 \\ 100.000 & 0.000 E+00 & 0.000 E+00 & 0.000 E+00 & 0.000 E+00 \\ 75.000 & 0.000 E+00 & 0.000 E+00 & 0.000 E+00 & 0.000 E+00 \\ 50.000 & 0.000 E+00 & 0.000 E+00 & 0.000 E+00 & 0.000 E+00 \\ 30.000 & 0.000 E+00 & 0.000 E+00 & 0.000 E+00 & 0.000 E+00 \\ 15.000 & 0.000 E+00 & 0.000 E+00 & 0.000 E+00 & 0.000 E+00 \\ 5.000 & 0.000 E+00 & 0.000 E+00 & 0.000 E+00 & 0.000 E+00 \\ 1.000 & 0.000 E+00 & 0.000 E+00 & 0.000 E+00 & 0.000 E+00 \\ 0.100 & 0.000 E+00 & 0.000 E+00 & 0.000 E+00 & 0.000 E+00 \\ 0.010 & 0.000 E+00 & 0.000 E+00 & 0.000 E+00 & 0.000 E+00 \\ 0.010 & 0.000 E+00 & 0.000 E+00 & 0.000 E+00 & 0.000 E+00\end{array}$


_INCIDENT-FREE_HAND_CHECK_RAIL_SUBURBAN_

DISPERSAL ACCIDENT INPUT

$\begin{array}{cc}\begin{array}{c}\text { AREADA } \\ \text { (M SQ) }\end{array} & \begin{array}{c}\text { DILUTION } \\ \text { FACTOR* }\end{array} \\ 4.590 E+02 & 3.420 E-03 \\ 1.530 E+03 & 1.720 E-03 \\ 3.940 E+03 & 8.580 E-04 \\ 1.250 E+04 & 3.420 E-04 \\ 3.040 E+04 & 1.720 E-04 \\ 6.850 E+04 & 8.580 E-05 \\ 1.760 E+05 & 3.420 E-05 \\ 4.450 E+05 & 1.720 E-05 \\ 8.590 E+05 & 8.580 E-06 \\ 2.550 E+06 & 3.420 E-06 \\ 4.450 E+06 & 1.720 E-06 \\ 1.030 E+07 & 8.580 E-07 \\ 2.160 E+07 & 3.420 E-07 \\ 5.520 E+07 & 1.720 E-07 \\ 1.770 E+08 & 8.580 E-08 \\ 4.890 E+08 & 5.420 E-08 \\ 8.120 E+08 & 4.300 E-08 \\ 1.350 E+09 & 3.420 E-08\end{array}$

* DILUTION FACTOR UNITS ARE (CI-SEC/M**3/CI-RELEASED)

NON-DISPERSAL ACCIDENT INPUT

$\begin{array}{lll} & \text { RADIST(M) } & \\ \text { RURAL } & \text { SUBURBAN } & \text { URBAN } \\ 3.050 E+00 & 3.050 E+00 & 3.050 E+00 \\ 6.100 E+00 & 6.100 E+00 & 6.100 E+00 \\ 9.100 E+00 & 9.100 E+00 & 9.100 E+00 \\ 1.220 E+01 & 1.220 E+01 & 1.220 E+01 \\ 1.520 E+01 & 1.520 E+01 & 1.520 E+01 \\ 3.050 E+01 & 3.050 E+01 & 3.050 E+01 \\ 6.100 E+01 & 6.100 E+01 & 6.100 E+01 \\ 9.140 E+01 & 9.140 E+01 & 9.140 E+01 \\ 1.524 E+02 & 1.524 E+02 & 1.524 E+02 \\ 3.050 E+02 & 3.050 E+02 & 3.050 E+02\end{array}$

BUILDING DOSE FACTOR

FRACTION OF LAND UNDER CULTIVATION

CONTAMINATION CLEAN UP LEVEL (UCI/M**2)

BREATHING RATE $(M * * 3 / S E C)$
$=8.600 \mathrm{E}-03$

$=4.400 E-01$

$=1.000 E+09$

$=3.300 E-04$ 
RUN DATE: [ 16-APR-95 AT 16:00:25 ]

_INCIDENT-FREE_HAND_CHECK_RAIL_SUBURBAN_

REGULATORY CHECKS

MODE 2 HAS BEEN REDESIGNATED AS EXCLUSIVE USE

FOR THE SHIPMENT OF SNF BY MODE 2

THE DOSE RATE AT 2 METERS COULD EXCEED 10 MR/HR

PPS*TI HAS BEEN RESET TO EQUAL 14.00 

_INCIDENT-FREE_HAND_CHECK_RAIL_SUBURBAN_

\author{
INCIDENT-FREE SUMMARY
}

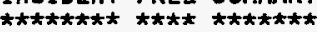

INCIDENT-FREE POPULATION EXPOSURE IN PERSON-REM

PASSENGR CREW HANDLERS OFF LINK ON LINK STOPS STORAGE TOTALS

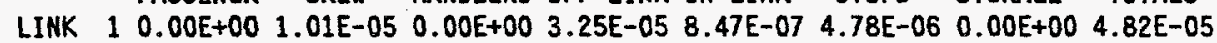

TOTALS: $0.00 E+00 \quad 1.01 E-05 \quad 0.00 E+00 \quad 3.25 E-05 \quad 8.47 E-07 \quad 4.78 E-06 \quad 0.00 E+00 \quad 4.82 E-05$

MAXIMUM INDIVIDUAL IN-TRANSIT DOSE

LINK $1 \quad 3.34$ E-07 REM 
_INCIDENT-FREE_HAND_CHECK_RAIL_SUBURBAN_

INCIDENT-FREE IMPORTANCE ANALYSIS SUMMARY FOR LINK 1

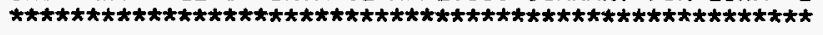

\section{INDEX}

DESCRIPTION OF PARAMETER

PACKAGES PER SHIPMENT

NUMBER OF SHIPMENTS

DOSE RATE (TRANSPORT INDEX)

K ZERO

DISTANCE TRAVELED

FRACTION OF TRAVEL - SUBURBAN

POPULATION DENSITY - SUBURBAN

STOP TIME

PERSONS EXPOSED WHILE STOPPED

NUMBER OF PEOPLE PER VEHICLE

TRAFFIC COUNT - SUBURBAN

TRAFFIC COUNT - URBAN

NUMBER OF HANDLINGS

NUMBER OF CREW MEMBERS

DISTANCE FROM SOURCE TO CREW

EXPOSURE TIME FOR HANDLERS

PERSONS EXPOSED PER HANDLING

FRACTION OF RUSH HOUR TRAVEL

NUMBER OF FLIGHT ATTENDANTS

TRAFFIC COUNT - RURAL

FRACTION OF TRAVEL ON FREEYAYS

HANDLER EXPOSURE DISTANCE

STORAGE EXPOSURE DISTANCE

NUMBER OF PERSONS EXPOSED DURING STORAGE

SUBURBAN SHIELDING FACTOR (RS)

RATIO OF PEDESTRIAN DENSITY (RPD)

VELOCITY - RURAL

POPULATION DENSITY - RURAL

FRACTION OF TRAVEL - RURAL

FRACTION OF TRAVEL - URBAN

POPULATION DENSITY - URBAN

STORAGE TIME PER SHIPMENT

URBAN SHIELDING FACTOR (RU)

FRACTION OF TRAVEL ON CITY STREETS

RURAL SHIELDING FACTOR (RR)

VELOCITY - URBAN

EXPOSURE DISTANCE WHILE STOPPED

VELOCITY - SUBURBAN
IMPORTANCE

4.821E-07

4.821E-07

4.821E-07

$4.821 E-07$

3.813E-07

$3.335 E-07$

$3.250 \mathrm{E}-07$

$4.775 \mathrm{E}-08$

4.775E-08

8.466E-09

8.466E-09

$0.000 E+00$

$0.000 E+00$

$0.000 E+00$

$0.000 E+00$

$0.000 E+00$

$0.000 E+00$

$0.000 E+00$

$0.000 E+00$

$0.000 E+00$

$0.000 E+00$

$0.000 E+00$

$0.000 \mathrm{E}+00$

$0.000 \mathrm{E}+00$

$0.000 \mathrm{E}+00$

$0.000 E+00$

$0.000 \mathrm{E}+00$

$0.000 E+00$

$0.000 E+00$

$0.000 E+00$

$0.000 E+00$

$0.000 E+00$

$0.000 E+00$

$0.000 E+00$

$0.000 E+00$

$0.000 E+00$

$-9.550 E-08$

$-3.420 E-07$

THE IMPORTANCE VALUE ESTIMATES THE PERSON-REM INFLUENCE OF A ONE PERCENT INCREASE IN THE PARAMETER

TOTAL EXPOSED POPULATION: INCIDENT-FREE
RAIL RURAL
$0.00 E+00$ PERSONS
RAIL SUBURBAN
$1.15 E+03$ PERSONS
RAIL URBAN
$0.00 E+00$ PERSONS
TOTAL 1.15E+03 PERSONS

TOTAL EXPOSED POPULATION: ACCIDENT

(PERSONS UNDER PLUME FOOTPRINT FOR A SINGLE ACCIDENT)

EOI
RAIL RURAL
$0.00 E+00$ PERSONS
RAIL SUBURBAN
$0.00 E+00$ PERSONS
RAIL URBAN
$0.00 E+00$ PERSONS

END OF RUN 


$$
\text { A-92 }
$$




\section{A.6 RURFU.OUT}

\section{UNIT RISK FACTORS FOR RAIL IN URBAN POPULATION ZONE}


A-94 


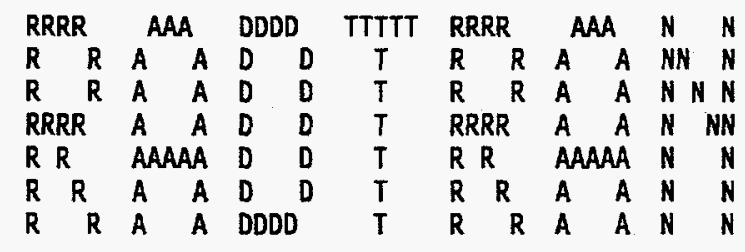

RADTRAN 4.0.17 VERSION DATE: NOVEMBER 8, 1994

MODE DESCRIPTIONS

$\begin{array}{cll}\text { NUMBER } & \text { NAME } & \text { CHARACTERIZATION } \\ 1 & \text { TRUCK } & \text { LONG HAUL VEHICLE } \\ 2 & \text { RAIL } & \text { COMHERCIAL TRAIN } \\ 3 & \text { BARGE } & \text { INLAND VESSEL } \\ 4 & \text { SHIP } & \text { OPEN SEA VESSEL } \\ 5 & \text { CARGO AIR } & \text { CARGO AIRCRAFT } \\ 6 & \text { PASS AIR } & \text { PASSENGER AIRCRAFT } \\ 7 & \text { P-VAN } & \text { PASSENGER VAN } \\ 8 & \text { CVAN-T } & \text { COMHERCIAL VAN } \\ 9 & \text { CVAN-R } & \text { COMMERCIAL VAN } \\ 10 & \text { CVAN-CA } & \text { COMMERCIAL VAN }\end{array}$


ECHO CHECK

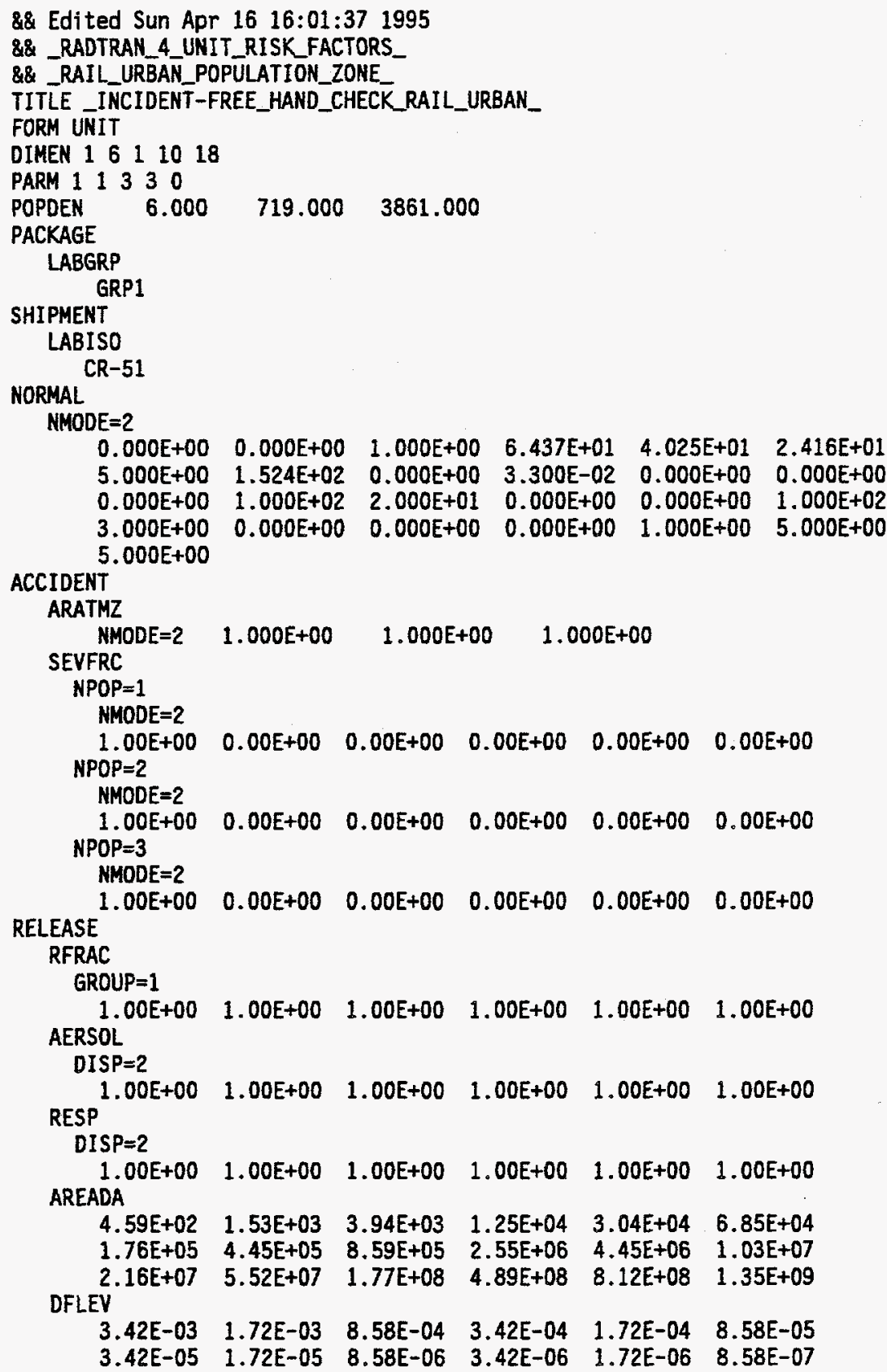


RUN DATE: [ 16-APR-95 AT 16:01:59 ]

_INCIDENT-FREE_HAND_CHECK_RAIL_URBAN_

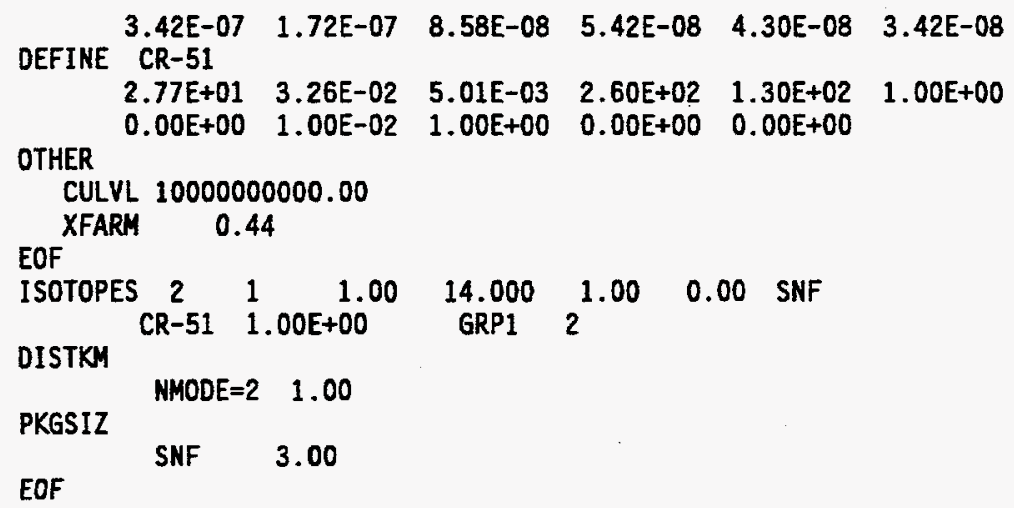


RUN DATE: [ 16-APR-95 AT 16:01:59]

_INCIDENT-FREE_HAND_CHECK_RAIL_URBAN_

\begin{tabular}{lc}
\multicolumn{1}{c}{ ZONE } & POPULATION DENSITY \\
& (PERSONS PER SQ KM) \\
RURAL & 6. \\
SUBURBAN & 719. \\
URBAN & 3861.
\end{tabular}

PACKAGE CHARACTERISTICS

$\begin{array}{cccc}\text { FOR } & \text { DIMENSION } & \text { EFFECTIVE } & K(0) \\ \text { MATERIAL } & \text { (METERS) } & \text { DIMENSION } & \text {. METERS SQ. } \\ \text { SNF } & 3.000 E+00 & 3.000 E+00 & 6.250 E+00\end{array}$

K(O) IS TI TO DOSE RATE CONVERSION FACTOR

PACKAGE HANDLING THRESHOLDS (METERS)

PKGSZ1 $=$ 5.000E-01

PKGSZ2 $=1.000 E+00$

PACKAGES . LE. PKGSZI ARE HAND CARRIED

PACKAGES .GT. PKGSZ1 AND .LE. PKGSZ2 ARE HANDLED BY SMALL EQUIPMENT

PACKAGES .GT. PKGSZ2 ARE HANDLED BY HEAVY EQUIPMENT

MATERIAL CHARACTERISTICS

$\begin{array}{rrr}\text { MATERIAL } & \text { FRACTION } & \text { FRACTION } \\ \text { SNF } & \text { OF GAMMA } & \text { OF NEUTRON } \\ & 1.000 E+00 & 0.000 E+00\end{array}$


RUN DATE: [ 16-APR-95 AT 16:01:59]

_INCIDENT-FREE_HAND_CHECK_RAIL_URBAN_

MODE CHARACTERISTICS

$\begin{array}{lrrrrrrr}\text { MODE } & \begin{array}{c}\text { OISTANCE } \\ \text { TRAVELED }\end{array} & \begin{array}{c}\text { EXCLUSIVE } \\ \text { USE }\end{array} & \begin{array}{c}\text { NUMBER OF } \\ \text { SHIPMENTS }\end{array} & \text { MATERIALS } & \begin{array}{c}\text { TRANSPORT } \\ \text { INDEX (TI) }\end{array} & \begin{array}{c}\text { PACKAGES/ } \\ \text { SHIPMENT }\end{array} \\ \text { RAIL } & 1.00 E+00 & \text { NO } & 1.00 E+00 & & & \\ & & & & \text { SNF } & 1.40 E+01 & 1.00 E+00\end{array}$

BUILDING SHIELDING OPTION= 3

(1=TOTAL SHIELDING, 2=PARTIAL SHIELDING, 3=NO SHIELDING)

$\mathrm{RPD}=6.000 \mathrm{E}+00$

(RATIO OF PEDESTRIAN DENSITY (PEDESTRIAN/KM SQ OF SIDEMALK)

TO POPULATION DENSITY (PEOPLE/KM SQ IN URBAN AREAS)

$R R=1.000 E+00$

(TRANSMISSION FACTOR FOR RURAL AREAS)

RS $=1.000 E+00$

(TRANSMISSION FACTOR FOR SUBURBAN AREAS)

$\mathrm{RU}=1.000 \mathrm{E}+00$

(TRANSMISSION FACTOR FOR URBAN AREAS) 
No

1 FRACTION OF TRAVEL IN RURAL POPULATION ZONE

2 FRACTION OF TRAVEL IN SUBURBAN POPULATION ZONE

3 FRACTION OF TRAVEL IN URBAN POPULATION ZONE

4. VELOCITY IN RURAL POPULATION ZONE (KILOMETERS/HOUR)

5 VELOCITY IN SUBURBAN POP. ZONE 4.025E+01 (KILOHETERS/HOUR)

6 VELOCITY IN URBAN POPULATION 2.416E+01 ZONE (KILOMETERS/HOUR)

7 NUMBER OF CREWMEN

$5.000 E+00$

8 DISTANCE FROM SOURCE TO CREW 1.524E+02 (METERS)

9 NUMBER OF HANDLINGS

$0.000 E+00$

10 STOP TIME PER KM (HR/KM)

3.300E-02

11 MINIMUM STOP TIME PER TRIP (HR)

12 ZERO STOP TIME PER TRIP (HR)

$0.000 E+00$

$0.000 E+00$

13 MINIMUM NUMBER OF RAIL CLASSIF $0.000 E+00$ ICATIONS/INSPECTIONS

14 PERSONS EXPOSED WHILE STOPPED 1.000E+02

15 AVERAGE EXPOSURE DISTANCE WHILE STOPPED (METERS)

16 STORAGE TIME PER SHIPMENT (HR)

17 MUMBER OF EXPOSED PERSONS DURING STORAGE

18 AVERAGE EXPOSURE DISTANCE WHILE IN STORAGE (METERS)

19 NUMBER OF PEOPLE PER VEHICLE ON LINK

20 FRACTION OF URBAN TRAVEL DURING RUSH HOUR TRAFFIC

21 FRACTION OF URBAN TRAVEL ON CITY STREETS

22 FRACTION OF RURAL-SUBURBAN TRAVEL ON FREEWAYS

23 *TRAFFIC COUNT PASSING A SPECIFIC POINT-RURAL ZONE

24 tTRAFFIC COUNT PASSING A SPECIFIC POINT-SUBURBAN ZONE

25 *TRAFFIC COUNT PASSING A SPECIFIC POINT-URBAN ZONE

* (ONE WAY VEHICLES/HR)
2.000E+01

$0.000 E+00$

$0.000 E+00$

$1.000 E+02$

$3.000 E+00$

$0.000 E+00$

$0.000 E+00$

$0.000 E+00$

$1.000 E+00$

$5.000 E+00$

$5.000 E+00$ 
RUN DATE: [ 16-APR-95 AT 16:01:59]

_INCIDENT-FREE_HAND_CHECK_RAIL_URBAN_

ISOTOPE RELATED DATA

\begin{tabular}{|c|c|c|c|c|c|c|c|}
\hline NUCLIDE & $\begin{array}{l}\text { CURIES } \\
\text { PER PKG }\end{array}$ & $\begin{array}{r}\text { RELEAS } \\
\text { GROUP }\end{array}$ & \multicolumn{2}{|c|}{$\begin{array}{l}\text { RESUSP } \\
\text { FACTOR }\end{array}$} & $\begin{array}{l}\text { LUNG } \\
\text { TYPE }\end{array}$ & $\begin{array}{l}\text { DISPERS. } \\
\text { CATEGORY }\end{array}$ & $\begin{array}{l}\text { IYR INHAL REM/CI } \\
\text { LUNG MARROW }\end{array}$ \\
\hline $\begin{array}{r}\text { SNF } \\
C R=51\end{array}$ & $1.00 E+00$ & GRP1 & \multicolumn{2}{|c|}{$1.32 E+00$} & 1 & 2 & $0.00 E+00 \quad 0.00 E+00$ \\
\hline NUCLIDE & $\begin{array}{l}\text { HALF } \\
\text { LIFE }\end{array}$ & $\begin{array}{l}\text { GAMMA } \\
\text { ENERGY }\end{array}$ & $\begin{array}{l}\text { CLOUD } \\
\text { FACTOR }\end{array}$ & & $\begin{array}{l}\text { TRAN } \\
\text { :ROPS }\end{array}$ & $\begin{array}{l}\text { SFER } \\
\text { SOIL }\end{array}$ & $\begin{array}{l}\text { DEPOS } \\
\text { SPEED }\end{array}$ \\
\hline $\begin{array}{r}\text { SNF } \\
\text { CR-51 }\end{array}$ & $2.77 E+01$ & $3.26 \mathrm{E}-02$ & $5.01 E-03$ & 1.00 & $00 E+00$ & $0.00 E+00$ & $1.00 E-02$ \\
\hline NUCLIDE & $\begin{array}{l}\text { 50-YR EF } \\
\text { INHAL }\end{array}$ & $\begin{array}{l}\text { FECTIVE RE } \\
\text { E INGES }\end{array}$ & & & & & \\
\hline $\begin{array}{r}\text { SNF } \\
\text { CR-51 }\end{array}$ & $2.60 \mathrm{E}+$ & $02 \quad 1.30 E+$ & & & & & \\
\hline
\end{tabular}


RUN DATE: [ 16-APR-95 AT 16:01:59]

_INCIDENT-FREE_HAND_CHECK_RAIL_URBAN_

RELEASE RELATED DATA

ACCIDENT RATES (PER KM)

MODE RURAL SUBURBAN URBAN

RAIL $1.000 \mathrm{E}+00 \quad 1.000 \mathrm{E}+00 \quad 1.000 \mathrm{E}+00$

RELEASE FRACTIONS

GROUP SEVER: 1 SEVER: 2 SEVER: 3 SEVER: 4 SEVER: 5 SEVER: 6

$1 \quad 1.00 E+00 \quad 1.00 E+00 \quad 1.00 E+00 \quad 1.00 E+00 \quad 1.00 E+00 \quad 1.00 E+00$

\section{ACCIDENT SEVERITY FRACTIONS}

$$
\text { FOR RAIL }
$$

ZONE SEVER: 1 SEVER: 2 SEVER: 3 SEVER: 4 SEVER: 5 SEVER: 6

$1 \quad 1.00 E+00 \quad 0.00 E+00 \quad 0.00 E+00 \quad 0.00 E+00 \quad 0.00 E+00 \quad 0.00 E+00$

$2 \quad 1.00 E+00 \quad 0.00 E+00 \quad 0.00 E+00 \quad 0.00 E+00 \quad 0.00 E+00 \quad 0.00 E+00$

$\begin{array}{lllllll}1.00 E+00 & 0.00 E+00 & 0.00 E+00 & 0.00 E+00 & 0.00 E+00 & 0.00 E+00\end{array}$ 
RUN DATE: [ 16-APR-95 AT 16:01:59]

_INCIDENT-FREE_HAND_CHECK_RAIL_URBAN_

AEROSOLIZEO FRACTION OF RELEASED MATERIAL

$\begin{array}{cllllll}\text { DISP CAT } & \text { SEVER: 1 } & \text { SEVER: 2 } & \text { SEVER: 3 } & \text { SEVER: } 4 & \text { SEVER: } 5 & \text { SEVER: } 6 \\ 1 & 0.00 E+00 & 0.00 E+00 & 0.00 E+00 & 0.00 E+00 & 0.00 E+00 & 0.00 E+00 \\ 2 & 1.00 E+00 & 1.00 E+00 & 1.00 E+00 & 1.00 E+00 & 1.00 E+00 & 1.00 E+00 \\ 3 & 1.00 E-02 & 1.00 E-02 & 1.00 E-02 & 1.00 E-02 & 1.00 E-02 & 1.00 E-02 \\ 4 & 5.00 E-02 & 5.00 E-02 & 5.00 E-02 & 5.00 E-02 & 5.00 E-02 & 5.00 E-02 \\ 5 & 1.00 E-01 & 1.00 E-01 & 1.00 E-01 & 1.00 E-01 & 1.00 E-01 & 1.00 E-01 \\ 6 & 1.00 E+00 & 1.00 E+00 & 1.00 E+00 & 1.00 E+00 & 1.00 E+00 & 1.00 E+00 \\ 7 & 1.00 E+00 & 1.00 E+00 & 1.00 E+00 & 1.00 E+00 & 1.00 E+00 & 1.00 E+00 \\ 8 & 1.00 E+00 & 1.00 E+00 & 1.00 E+00 & 1.00 E+00 & 1.00 E+00 & 1.00 E+00 \\ 9 & 1.00 E+00 & 1.00 E+00 & 1.00 E+00 & 1.00 E+00 & 1.00 E+00 & 1.00 E+00 \\ 10 & 1.00 E+00 & 1.00 E+00 & 1.00 E+00 & 1.00 E+00 & 1.00 E+00 & 1.00 E+00 \\ 11 & 1.00 E+00 & 1.00 E+00 & 1.00 E+00 & 1.00 E+00 & 1.00 E+00 & 1.00 E+00\end{array}$

FRACTION OF AEROSOLS BELOW 10 MICRONS AED

$\begin{array}{ccccccc}\text { DISP CAT } & \text { SEVER: } 1 & \text { SEVER: } 2 & \text { SEVER: 3 } & \text { SEVER: } 4 & \text { SEVER: } 5 & \text { SEVER: } 6 \\ 1 & 0.00 E+00 & 0.00 E+00 & 0.00 E+00 & 0.00 E+00 & 0.00 E+00 & 0.00 E+00 \\ 2 & 1.00 E+00 & 1.00 E+00 & 1.00 E+00 & 1.00 E+00 & 1.00 E+00 & 1.00 E+00 \\ 3 & 5.00 E-02 & 5.00 E-02 & 5.00 E-02 & 5.00 E-02 & 5.00 E-02 & 5.00 E-02 \\ 4 & 5.00 E-02 & 5.00 E-02 & 5.00 E-02 & 5.00 E-02 & 5.00 E-02 & 5.00 E-02 \\ 5 & 5.00 E-02 & 5.00 E-02 & 5.00 E-02 & 5.00 E-02 & 5.00 E-02 & 5.00 E-02 \\ 6 & 5.00 E-02 & 5.00 E-02 & 5.00 E-02 & 5.00 E-02 & 5.00 E-02 & 5.00 E-02 \\ 7 & 1.00 E+00 & 1.00 E+00 & 1.00 E+00 & 1.00 E+00 & 1.00 E+00 & 1.00 E+00 \\ 8 & 1.00 E+00 & 1.00 E+00 & 1.00 E+00 & 1.00 E+00 & 1.00 E+00 & 1.00 E+00 \\ 9 & 1.00 E+00 & 1.00 E+00 & 1.00 E+00 & 1.00 E+00 & 1.00 E+00 & 1.00 E+00 \\ 10 & 1.00 E+00 & 1.00 E+00 & 1.00 E+00 & 1.00 E+00 & 1.00 E+00 & 1.00 E+00 \\ 11 & 1.00 E+00 & 1.00 E+00 & 1.00 E+00 & 1.00 E+00 & 1.00 E+00 & 1.00 E+00\end{array}$


RUN DATE: [ 16-APR-95 AT 16:01:59]

_INCIDENT-FREE_HAND_CHECK_RAIL_URBAN_

COST RELATED DATA

EMERGENCY RESPONSE COST

SEVER: 1 SEVER: 2 SEVER: 3 SEVER: 4 SEVER: 5 SEVER: 6

$0.00 E+00 \quad 0.00 E+00 \quad 0.00 E+00 \quad 0.00 E+00 \quad 0.00 E+00 \quad 0.00 E+00$

ON-SCENE COSTS

(RF=RELEASE FRACTION)

$R F=0 . \quad 0 .<R F<=.01 \quad .01<R F<=0.1 \quad .1<R F<=1$.
0 .
0 .
0 .
0 . 
RUN DATE: [ 16-APR-95 AT 16:01:59]

_INCIDENT-FREE_HAND_CHECK_RAIL_URBAN_

HEALTH RELATED DATA

\section{EARLY FATALITY PROBABILITIES}

$\begin{array}{rrrrr}\text { DOSE (REM) } & \text { LUNG-1 } & \text { LUNG-2 } & \text { LUNG-3 } & \text { MARROW } \\ 100000.000 & 1.000 E+00 & 1.000 E+00 & 1.000 E+00 & 1.000 E+00 \\ 80000.000 & 1.000 E+00 & 8.500 E-01 & 8.000 E-01 & 1.000 E+00 \\ 70000.000 & 1.000 E+00 & 8.000 E-01 & 5.000 E-01 & 1.000 E+00 \\ 40000.000 & 1.000 E+00 & 7.000 E-01 & 0.000 E+00 & 1.000 E+00 \\ 30000.000 & 1.000 E+00 & 5.000 E-01 & 0.000 E+00 & 1.000 E+00 \\ 25000.000 & 1.000 E+00 & 2.000 E-01 & 0.000 E+00 & 1.000 E+00 \\ 20000.000 & 1.000 E+00 & 8.000 E-02 & 0.000 E+00 & 1.000 E+00 \\ 10000.000 & 6.000 E-01 & 0.000 E+00 & 0.000 E+00 & 1.000 E+00 \\ 8000.000 & 1.000 E-01 & 0.000 E+00 & 0.000 E+00 & 1.000 E+00 \\ 6000.000 & 6.000 E-02 & 0.000 E+00 & 0.000 E+00 & 1.000 E+00 \\ 4000.000 & 3.000 E-02 & 0.000 E+00 & 0.000 E+00 & 1.000 E+00 \\ 3000.000 & 0.000 E+00 & 0.000 E+00 & 0.000 E+00 & 1.000 E+00 \\ 2000.000 & 0.000 E+00 & 0.000 E+00 & 0.000 E+00 & 1.000 E+00 \\ 1000.000 & 0.000 E+00 & 0.000 E+00 & 0.000 E+00 & 1.000 E+00 \\ 800.000 & 0.000 E+00 & 0.000 E+00 & 0.000 E+00 & 9.960 E-01 \\ 700.000 & 0.000 E+00 & 0.000 E+00 & 0.000 E+00 & 9.000 E-01 \\ 600.000 & 0.000 E+00 & 0.000 E+00 & 0.000 E+00 & 4.000 E-01 \\ 500.000 & 0.000 E+00 & 0.000 E+00 & 0.000 E+00 & 5.000 E-02 \\ 400.000 & 0.000 E+00 & 0.000 E+00 & 0.000 E+00 & 0.000 E+00 \\ 300.000 & 0.000 E+00 & 0.000 E+00 & 0.000 E+00 & 0.000 E+00 \\ 100.000 & 0.000 E+00 & 0.000 E+00 & 0.000 E+00 & 0.000 E+00 \\ 75.000 & 0.000 E+00 & 0.000 E+00 & 0.000 E+00 & 0.000 E+00 \\ 50.000 & 0.000 E+00 & 0.000 E+00 & 0.000 E+00 & 0.000 E+00 \\ 30.000 & 0.000 E+00 & 0.000 E+00 & 0.000 E+00 & 0.000 E+00 \\ 15.000 & 0.000 E+00 & 0.000 E+00 & 0.000 E+00 & 0.000 E+00 \\ 5.000 & 0.000 E+00 & 0.000 E+00 & 0.000 E+00 & 0.000 E+00 \\ 1.000 & 0.000 E+00 & 0.000 E+00 & 0.000 E+00 & 0.000 E+00 \\ 0.100 & 0.000 E+00 & 0.000 E+00 & 0.000 E+00 & 0.000 E+00 \\ 0.010 & 0.000 E+00 & 0.000 E+00 & 0.000 E+00 & 0.000 E+00 \\ 0.010 & 0.000 E+00 & 0.000 E+00 & 0.000 E+00 & 0.000 E+00\end{array}$


_INCIDENT-FREE_HAND_CHECK_RAIL_URBAN_

DISPERSAL ACCIDENT INPUT

$\begin{array}{cc}\begin{array}{c}\text { AREADA } \\ \text { (M SQ) }\end{array} & \begin{array}{c}\text { DILUTION } \\ \text { FACTOR* }\end{array} \\ 4.590 E+02 & 3.420 E-03 \\ 1.530 E+03 & 1.720 E-03 \\ 3.940 E+03 & 8.580 E-04 \\ 1.250 E+04 & 3.420 E-04 \\ 3.040 E+04 & 1.720 E-04 \\ 6.850 E+04 & 8.580 E-05 \\ 1.760 E+05 & 3.420 E-05 \\ 4.450 E+05 & 1.720 E-05 \\ 8.590 E+05 & 8.580 E-06 \\ 2.550 E+06 & 3.420 E-06 \\ 4.450 E+06 & 1.720 E-06 \\ 1.030 E+07 & 8.580 E-07 \\ 2.160 E+07 & 3.420 E-07 \\ 5.520 E+07 & 1.720 E-07 \\ 1.770 E+08 & 8.580 E-08 \\ 4.890 E+08 & 5.420 E-08 \\ 8.120 E+08 & 4.300 E-08 \\ 1.350 E+09 & 3.420 E-08\end{array}$

* Dilution Factor UNITS ARE (CI-SEC/M**3/CI-RELEASED)

NON-DISPERSAL ACCIDENT INPUT

$\begin{array}{lll}\text { RADIST(M) } & \\ \text { RURAL } & \text { SUBURBAN } & \text { URBAN } \\ 3.050 E+00 & 3.050 E+00 & 3.050 E+00 \\ 6.100 E+00 & 6.100 E+00 & 6.100 E+00 \\ 9.100 E+00 & 9.100 E+00 & 9.100 E+00 \\ 1.220 E+01 & 1.220 E+01 & 1.220 E+01 \\ 1.520 E+01 & 1.520 E+01 & 1.520 E+01 \\ 3.050 E+01 & 3.050 E+01 & 3.050 E+01 \\ 6.100 E+01 & 6.100 E+01 & 6.100 E+01 \\ 9.140 E+01 & 9.140 E+01 & 9.140 E+01 \\ 1.524 E+02 & 1.524 E+02 & 1.524 E+02 \\ 3.050 E+02 & 3.050 E+02 & 3.050 E+02\end{array}$

BUILDING DOSE FACTOR

FRACTION OF LAND UNDER CULTIVATION

CONTAMINATION CLEAN UP LEVEL (UCI/ $/ M^{\star *} 2$ )

BREATHING RATE ( $M * \star 3 / S E C)$
$=8.600 \mathrm{E}-03$

$=4.400 E-01$

$=1.000 E+09$

$=3.300 E-04$ 
RUN DATE: [ 16-APR-95 AT 16:01:59]

_INCIDENT-FREE_HAND_CHECK_RAIL_URBAN_

REGULATORY CHECKS

MODE 2 HAS BEEN REDESIGNATED AS EXCLUSIVE USE

FOR THE SHIPMENT OF

SHF BY MODE 2

THE DOSE RATE AT 2 METERS COULD EXCEED 10 MR/HR

PPS $\star T I$ HAS BEEN RESET TO EQUAL 14.00 
RUN DATE: [ 16-APR-95 AT 16:01:59]

_INCIDENT-FREE_HAND_CHECK_RAIL_URBAN_

$$
\begin{aligned}
& \text { INCIDENT-FREE SUMMARY }
\end{aligned}
$$

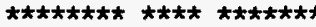

INCIDENT-FREE POPULATION EXPOSURE IN PERSON-REM

PASSENGR CREW HANDLERS OFF LINK ON LINK STOPS STORAGE TOTALS LINK $10.00 E+00 \quad 1.01 E-05 \quad 0.00 E+00 \quad 2.91 E-04 \quad 2.35 E-06 \quad 4.78 E-06 \quad 0.00 E+00 \quad 3.08 E-04$

TOTALS: $0.00 E+00 \quad 1.01 E-05 \quad 0.00 E+00 \quad 2.91 E-04 \quad 2.35 E-06 \quad 4.78 E-06 \quad 0.00 E+00 \quad 3.08 E-04$

MAXIMUM INDIVIDUAL IN-TRANSIT DOSE

LINK 1 3.34E-07 REM 
_INCIDENT-FREE_HAND_CHECK_RAIL_URBAN_

INCIDENT-FREE IMPORTANCE ANALYSIS SUMMARY FOR LINK 1

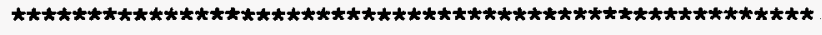

INDEX

1 K ZERO

2 NUMBER OF SHIPMENTS

3 PACKAGES PER SHIPMENT

4 DOSE RATE (TRANSPORT INDEX)

5 DISTANCE TRAVELED

6 FRACTION OF TRAVEL - URBAN

7 POPULATION DENSITY - URBAN

8 STOP TIME

9 PERSONS EXPOSED WHILE STOPPED

10 NUMBER OF PEOPLE PER VEHICLE

11 TRAFFIC COUNT - URBAN

12 NUMBER OF CREN MEMBERS

13 EXPOSURE TIME FOR HANDLERS

14 NUMBER OF HANDLINGS

15 DISTANCE FROM SOURCE TO CREW

16 PERSONS EXPOSED PER HANDLING

17 HANDLER EXPOSURE DISTANCE

18 NUMBER OF FLIGHT ATTENDANTS

19 TRAFFIC COUNT - SUBURBAN

20 FRACTION OF RUSH HOUR TRAVEL

21 TRAFFIC COUNT - RURAL

22 FRACTION OF TRAVEL ON FREEWAYS

23 STORAGE EXPOSURE DISTANCE

24 NUMBER OF PERSONS EXPOSED DURING STORAGE

25 FRACTION OF TRAVEL - SUBURBAN

26 RATIO OF PEDESTRIAN DENSITY (RPD)

27 VELOCITY - RURAL

28 POPULATION DENSITY - RURAL

29 FRACTION OF TRAVEL - RURAL

30 POPULATION DENSITY - SUBURBAN

31 VELOCITY - SUBURBAN

32 SUBURBAN SHIELDING FACTOR (RS)

33 STORAGE TIME PER SHIPMENT

34 URBAN SHIELDING FACTOR (RU)

35 FRACTION OF TRAVEL ON CITY STREETS

36 RURAL SHIELDING FACTOR (RR)

37 EXPOSURE DISTANCE WHILE STOPPED

38 VELOCITY - URBAN
IMPORTANCE

$3.080 E-06$

$3.080 E-06$

$3.080 \mathrm{E}-06$

$3.080 \mathrm{E}-06$

2.979E-06

2.931E-06

$2.908 \mathrm{E}-06$

4. $775 \mathrm{E}-08$

4.775E-08

2.350E-08

$2.350 E-08$

$0.000 E+00$

$0.000 E+00$

$0.000 E+00$

$0.000 E+00$

$0.000 E+00$

$0.000 E+00$

$0.000 E+00$

$0.000 \mathrm{E}+00$

$0.000 E+00$

$0.000 E+00$

$0.000 E+00$

$0.000 E+00$

$0.000 E+00$

$0.000 E+00$

$0.000 E+00$

$0.000 \mathrm{E}+00$

$0.000 E+00$

$0.000 E+00$

$0.000 E+00$

$0.000 E+00$

$0.000 E+00$

$0.000 E+00$

$0.000 E+00$

$0.000 E+00$

$0.000 E+00$

$-9.550 E-08$

$-2.955 E-06$

THE IMPORTANCE VALUE ESTIMATES THE PERSON-REM INFLUENCE OF A ONE PERCENT INCREASE IN THE PARAMETER

TOTAL EXPOSED POPULATION: INCIDENT-FREE
RAIL RURAL
$0.00 E+00$ PERSONS
RAIL SUBURBAN
$0.00 E+00$ PERSOHS
RAIL URBAN
$6.18 E+03$ PERSONS
TOTAL $6.18 E+03$ PERSONS

TOTAL EXPOSED POPULATION: ACCIDENT

(PERSONS UNOER PLUME FOOTPRINT FOR A SINGLE ACCIDENT)

EOI
RAIL RURAL
$0.00 E+00$ PERSONS
RAIL SUBURBAN
$0.00 E+00$ PERSONS
RAIL URBAN
$0.00 E+00$ PERSONS

END OF RUN 
A-110 


\section{A.7 RURFN.OUT \\ NONLINEAR UNIT RISK FACTORS FOR RAIL IN ALL POPULATION ZONES}


A-112 


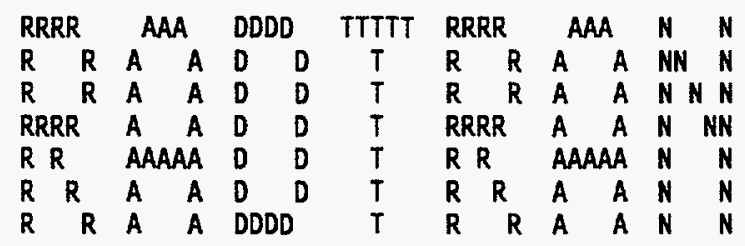

RADTRAN 4.0.17 VERSION DATE: NOVEMBER 8, 1994

MODE DESCRIPTIONS

$\begin{array}{cll}\text { NUMBER } & \text { NAME } & \text { CHARACTERIZATION } \\ 1 & \text { TRUCK } & \text { LONG HAUL VEHICLE } \\ 2 & \text { RAIL } & \text { COMMERCIAL TRAIN } \\ 3 & \text { BARGE } & \text { INLAND VESSEL } \\ 4 & \text { SHIP } & \text { OPEN SEA VESSEL } \\ 5 & \text { CARGO AIR } & \text { CARGO AIRCRAFT } \\ 6 & \text { PASS AIR } & \text { PASSENGER AIRCRAFT } \\ 7 & \text { P-VAN } & \text { PASSENGER VAN } \\ 8 & \text { CVAN-T } & \text { COMNERCIAL VAN } \\ 9 & \text { CVAN-R } & \text { COMNERCIAL VAN } \\ 10 & \text { CVAN-CA } & \text { COMMERCIAL VAN }\end{array}$




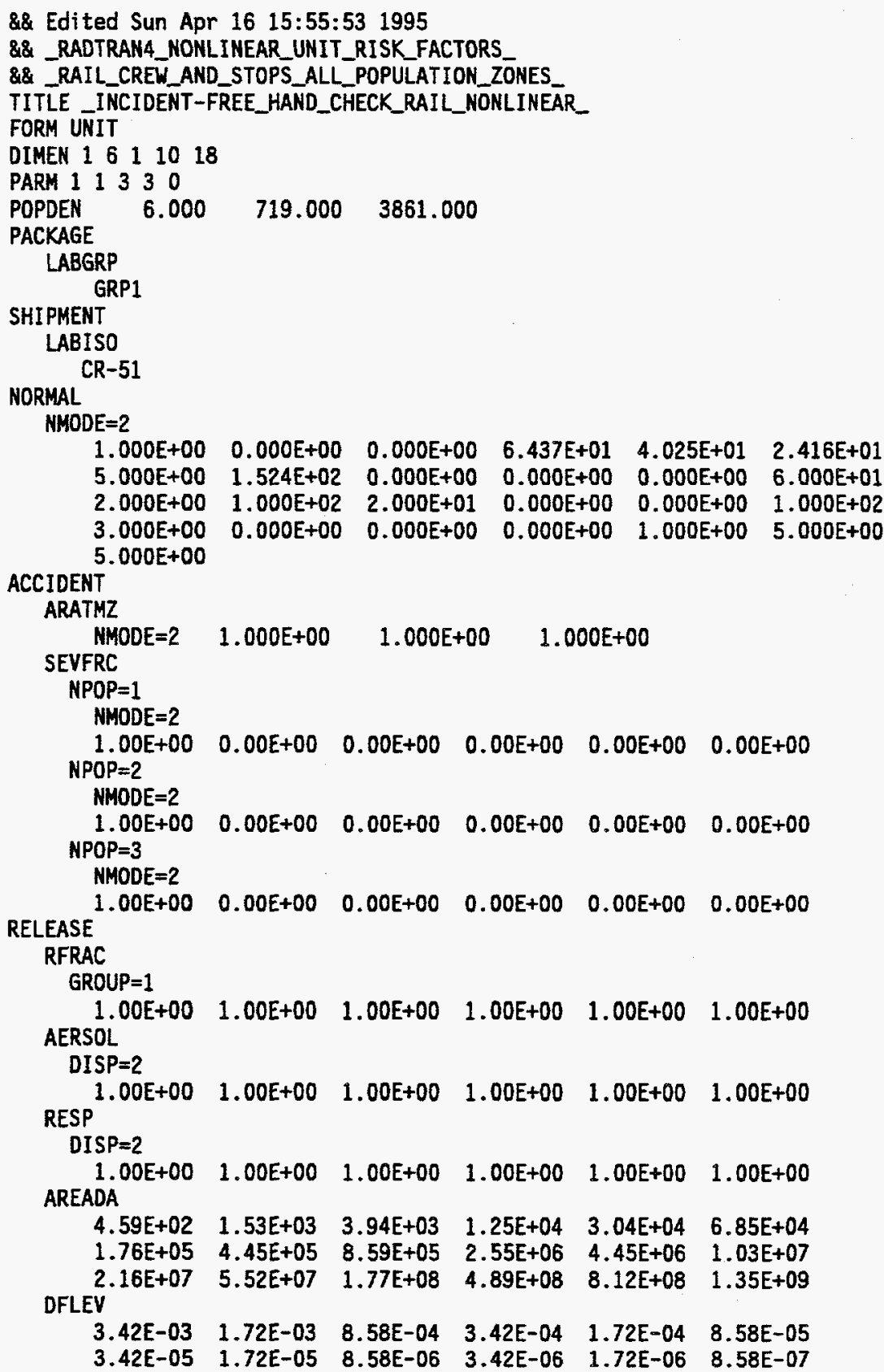


_INCIDENT-FREE_HAND_CHECK_RAIL_NONLINEAR_

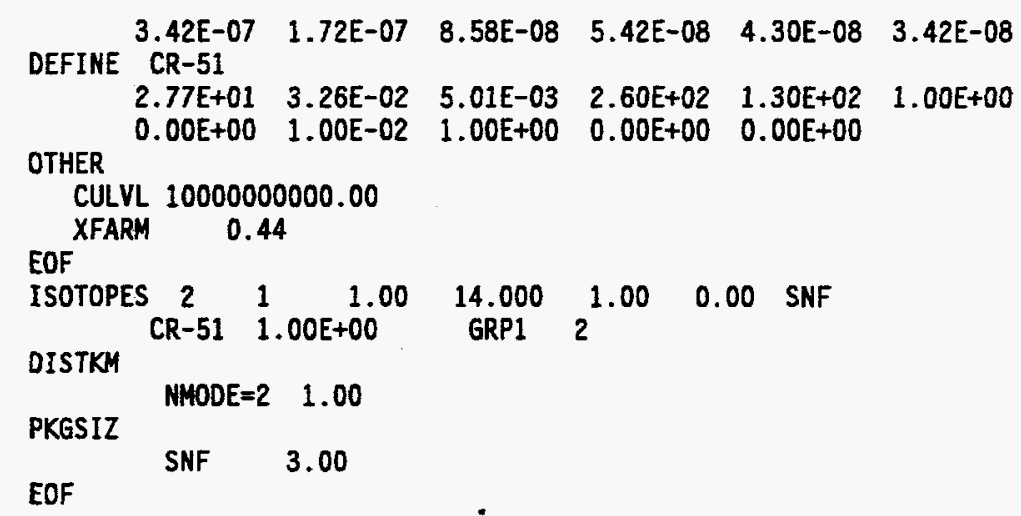


RUN DATE: [ 16-APR-95 AT 15:56:13 ]

_INCIDENT-FREE_HAND_CHECK_RAIL_NONLINEAR_

\begin{tabular}{lc}
\multicolumn{1}{c}{ ZONE } & POPULATION DENSITY \\
& (PERSONS PER SQ KM) \\
RURAL & 6. \\
SUBURBAN & 719. \\
URBAN & 3861.
\end{tabular}

PACKAGE CHARACTERISTICS

$\begin{array}{cccc}\text { FOR } & \text { DIMENSION } & \text { EFFECTIVE } & \text { K(0) } \\ \text { MATERIAL } & \text { (METERS) } & \text { DIMENSION } & \text { METERS SQ. } \\ \text { SNF } & 3.000 E+00 & 3.000 E+00 & 6.250 E+00\end{array}$

$K(0)$ IS TI TO DOSE RATE CONVERSION FACTOR

PACKAGE HANDLING THRESHOLDS (METERS)

PKGSZ1 $=5.000 E-01$

PKGSZ2 $=1.000 E+00$

PACKAGES .LE. PKGSZ1 ARE HAND CARRIED

PACKAGES .GT. PKGSZ1 AND .LE. PKGSZ2 ARE HANDLED BY SMALL EQUIPMENT

PACKAGES .GT. PKGSZZ ARE HANDLED BY HEAVY EQUIPMENT

MATERIAL CHARACTERISTICS

$\begin{array}{rrr} & \text { FRACTION } & \text { FRACTION } \\ \text { MATERIAL } & \text { OF GAMMA } & \text { OF NEUTRON } \\ \text { SNF } & 1.000 E+00 & 0.000 E+00\end{array}$


RUN DATE: [ 16-APR-95 AT 15:56:13 ]

_INCIDENT-FREE_HAND_CHECK_RAIL_MONLINEAR_

MODE CHARACTERISTICS

$\begin{array}{lrrrrrr}\text { MODE } & \begin{array}{c}\text { DISTANCE } \\ \text { TRAVELED }\end{array} & \begin{array}{c}\text { EXCLUSIVE } \\ \text { USE }\end{array} & \begin{array}{c}\text { NUMBER OF } \\ \text { SHIPMENTS }\end{array} & \text { MATERIALS } & \begin{array}{c}\text { TRANSPORT } \\ \text { INDEX (TI) }\end{array} & \begin{array}{c}\text { PACKAGES/ } \\ \text { SHIPMENT }\end{array} \\ \text { RAIL } & 1.00 E+00 & \text { NO } & 1.00 E+00 & & & \\ & & & & \text { SNF } & 1.40 E+01 & 1.00 E+00\end{array}$

BUILDING SHIELDING OPTION= 3

(1=TOTAL SHIELDING, 2=PARTIAL SHIELDING, 3=NO SHIELDING)

$R P D=6.000 E+00$

(RATIO OF PEDESTRIAN DENSITY (PEDESTRIAN/KM SQ OF SIDEMALK)

TO POPULATION DENSITY (PEOPLE/KY SQ IN URBAN AREAS)

$\mathrm{RR}=1.000 \mathrm{E}+00$

(TRANSMISSION FACTOR FOR RURAL AREAS)

RS $=1.000 E+00$

(TRANSMISSION FACTOR FOR SUBURBAN AREAS)

$R U=1.000 E+00$

(TRANSMISSION FACTOR FOR URBAN AREAS) 
NO

1 FRACTION OF TRAVEL

IN RURAL POPULATION ZONE

2 FRACTION OF TRAVEL IN SUBURBAN POPULATION ZONE

3 FRACTION OF TRAVEL IN URBAN POPULATION ZONE

4 VELOCITY IN RURAL POPULATION ZONE (KILONETERS/HOUR)

5 VELOCITY IN SUBURBAN POP. ZONE 4.025E+01 (KILOMETERS/HOUR)

6 VELOCITY IN URBAN POPULATION 2.416E+01 ZONE (KILOMETERS/HOUR)

7 NUMBER OF CREMMEN

$5.000 E+00$

8 DISTANCE FROM SOURCE TO CREN 1.524E+02 (METERS)

9 NUMBER OF HANDLINGS $0.000 E+00$

10 STOP TIME PER KM (HR/KM) $0.000 E+00$

11 MINIMUM STOP TIME PER TRIP $0.000 E+00$ (HR)

12 ZERO STOP TIME PER TRIP (HR) 6.000E+01

13 MINIMUM NUMBER OF RAIL CLASSIF 2.000E+00 ICATIONS/INSPECTIONS

14 PERSONS EXPOSED WHILE STOPPED $1.000 E+02$

15 AVERAGE EXPOSURE DISTANCE WHILE STOPPED (METERS)

16 STORAGE TIME PER SHIPMENT (HR)

17 NUMBER OF EXPOSED PERSONS DURING STORAGE

18 AVERAGE EXPOSURE DISTANCE WHILE IN STORAGE (METERS)

19 NUMBER OF PEOPLE PER VEHICLE ON LINK

20 FRACTION OF URBAN TRAVEL DURING RUSH HOUR TRAFFIC

21 FRACTION OF URBAN TRAVEL ON CITY STREETS

22 FRACTION OF RURAL-SUBURBAN TRAVEL ON FREEWAYS

23 *TRAFFIC COUNT PASSING A SPECIFIC POINT-RURAL ZONE

24 *TRAFFIC COUNT PASSING A SPECIFIC POINT-SUBURBAN ZONE

25 *TRAFFIC COUNT PASSING A SPECIFIC POINT-URBAN ZONE

* (ONE WAY VEHICLES/HR)
$2.000 E+01$

$0.000 \mathrm{E}+00$

$0.000 E+00$

$1.000 E+02$

3. $000 E+00$

$0.000 E+00$

$0.000 E+00$

$0.000 E+\infty 0$

$1.000 E+00$

$5.000 E+00$

$5.000 E+00$ 
RUN DATE: [ 16-APR-95 AT 15:56:13 ]

PAGE 7

_INCIDENT-FREE_HAND_CHECK_RAIL_NONLINEAR_

ISOTOPE RELATED DATA

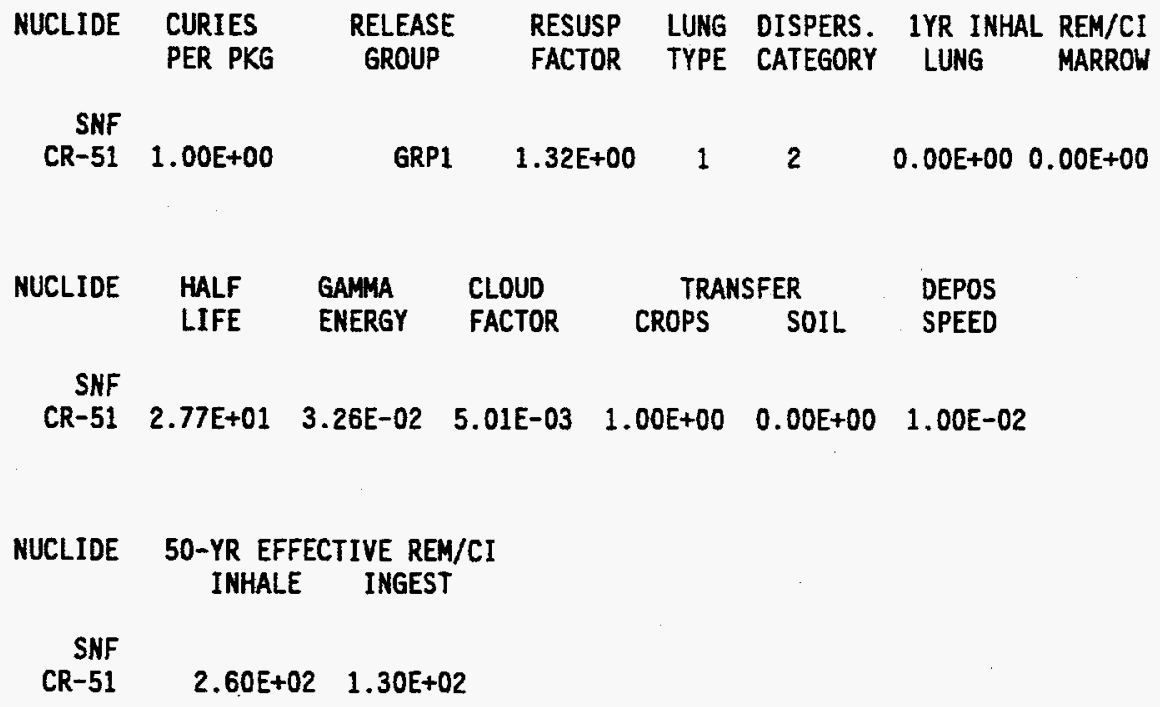


RUN DATE: [ 16-APR-95 AT 15:56:13 ]

_INCIDENT-FREE_HAND_CHECK_RAIL_NONLINEAR_

RELEASE RELATED DATA

ACCIDENT RATES (PER KM)

MODE RURAL SUBURBAN URBAN

RAIL $\quad 1.000 E+00 \quad 1.000 E+00 \quad 1.000 E+00$

RELEASE FRACTIONS

GROUP SEVER: 1 SEVER: 2 SEVER: 3 SEVER: 4 SEVER: 5 SEVER: 6

$1 \quad 1.00 E+00 \quad 1.00 E+00 \quad 1.00 E+00 \quad 1.00 E+00 \quad 1.00 E+00 \quad 1.00 E+00$

ACCIDENT SEVERITY FRACTIONS

FOR RAIL

ZONE SEVER: 1 SEVER: 2 SEVER: 3 SEVER: 4 SEVER: 5 SEVER: 6

$1 \quad 1.00 E+00 \quad 0.00 E+00 \quad 0.00 E+00 \quad 0.00 E+00 \quad 0.00 E+00 \quad 0.00 E+00$

$2 \quad 1.00 E+00 \quad 0.00 E+00 \quad 0.00 E+00 \quad 0.00 E+00 \quad 0.00 E+00 \quad 0.00 E+00$

$3 \quad 1.00 E+00 \quad 0.00 E+00 \quad 0.00 E+00 \quad 0.00 E+00 \quad 0.00 E+00 \quad 0.00 E+00$ 
_INCIDENT-FREE_HAND_CHECK_RAIL_NONLINEAR_

AEROSOLIZEO FRACTION OF RELEASEO MATERIAL

$\begin{array}{cllllll}\text { DISP CAT } & \text { SEVER: } 1 & \text { SEVER: } 2 & \text { SEVER: } 3 & \text { SEVER: } 4 & \text { SEVER: } 5 & \text { SEVER: } 6 \\ 1 & 0.00 E+00 & 0.00 E+00 & 0.00 E+00 & 0.00 E+00 & 0.00 E+00 & 0.00 E+00 \\ 2 & 1.00 E+00 & 1.00 E+00 & 1.00 E+00 & 1.00 E+00 & 1.00 E+00 & 1.00 E+00 \\ 3 & 1.00 E-02 & 1.00 E-02 & 1.00 E-02 & 1.00 E-02 & 1.00 E-02 & 1.00 E-02 \\ 4 & 5.00 E-02 & 5.00 E-02 & 5.00 E-02 & 5.00 E-02 & 5.00 E-02 & 5.00 E-02 \\ 5 & 1.00 E-01 & 1.00 E-01 & 1.00 E-01 & 1.00 E-01 & 1.00 E-01 & 1.00 E-01 \\ 6 & 1.00 E+00 & 1.00 E+00 & 1.00 E+00 & 1.00 E+00 & 1.00 E+00 & 1.00 E+00 \\ 7 & 1.00 E+00 & 1.00 E+00 & 1.00 E+00 & 1.00 E+00 & 1.00 E+00 & 1.00 E+00 \\ 8 & 1.00 E+00 & 1.00 E+00 & 1.00 E+00 & 1.00 E+00 & 1.00 E+00 & 1.00 E+00 \\ 9 & 1.00 E+00 & 1.00 E+00 & 1.00 E+00 & 1.00 E+00 & 1.00 E+00 & 1.00 E+00 \\ 10 & 1.00 E+00 & 1.00 E+00 & 1.00 E+00 & 1.00 E+00 & 1.00 E+00 & 1.00 E+00 \\ 11 & 1.00 E+00 & 1.00 E+00 & 1.00 E+00 & 1.00 E+00 & 1.00 E+00 & 1.00 E+00\end{array}$

FRACTION OF AEROSOLS BELOW 10 MICRONS AED

$\begin{array}{ccccccc}\text { DISP CAT } & \text { SEVER: } 1 & \text { SEVER: } 2 & \text { SEVER: } 3 & \text { SEVER: } 4 & \text { SEVER: } 5 & \text { SEVER: } 6 \\ 1 & 0.00 E+00 & 0.00 E+00 & 0.00 E+00 & 0.00 E+00 & 0.00 E+00 & 0.00 E+00 \\ 2 & 1.00 E+00 & 1.00 E+00 & 1.00 E+00 & 1.00 E+00 & 1.00 E+00 & 1.00 E+00 \\ 3 & 5.00 E-02 & 5.00 E-02 & 5.00 E-02 & 5.00 E-02 & 5.00 E-02 & 5.00 E-02 \\ 4 & 5.00 E-02 & 5.00 E-02 & 5.00 E-02 & 5.00 E-02 & 5.00 E-02 & 5.00 E-02 \\ 5 & 5.00 E-02 & 5.00 E-02 & 5.00 E-02 & 5.00 E-02 & 5.00 E-02 & 5.00 E-02 \\ 6 & 5.00 E-02 & 5.00 E-02 & 5.00 E-02 & 5.00 E-02 & 5.00 E-02 & 5.00 E-02 \\ 7 & 1.00 E+00 & 1.00 E+00 & 1.00 E+00 & 1.00 E+00 & 1.00 E+00 & 1.00 E+00 \\ 8 & 1.00 E+00 & 1.00 E+00 & 1.00 E+00 & 1.00 E+00 & 1.00 E+00 & 1.00 E+00 \\ 9 & 1.00 E+00 & 1.00 E+00 & 1.00 E+00 & 1.00 E+00 & 1.00 E+00 & 1.00 E+00 \\ 10 & 1.00 E+00 & 1.00 E+00 & 1.00 E+00 & 1.00 E+00 & 1.00 E+00 & 1.00 E+00 \\ 11 & 1.00 E+00 & 1.00 E+00 & 1.00 E+00 & 1.00 E+00 & 1.00 E+00 & 1.00 E+00\end{array}$


RUN DATE: [ 16-APR-95 AT 15:56:13 ]

_INCIDENT-FREE_HAND_CHECK_RAIL_MONLINEAR_

COST RELATED DATA

\section{EMERGENCY RESPONSE COST}

SEVER: 1 SEVER: 2 SEVER: 3 SEVER: 4 SEVER: 5 SEVER: 6

1

$\begin{array}{llllll}0.00 E+00 & 0.00 E+00 & 0.00 E+00 & 0.00 E+00 & 0.00 E+00 & 0.00 E+00\end{array}$

ON-SCENE COSTS

(RF=RELEASE FRACTION)

$R F=0 . \quad 0 .<R F<=.01 \quad .01<R F<=0.1 \quad .1<R F<=1$.
0.
0 .
0.
0 . 
RUN DATE: [ 16-APR-95 AT 15:56:13]

_INCIDENT-FREE_HAND_CHECK_RAIL_NONLINEAR_

HEALTH RELATED DATA

\section{EARLY FATALITY PROBABILITIES}

$\begin{array}{rrrrr}\text { DOSE(REM) } & \text { LUNG-1 } & \text { LUNG-2 } & \text { LUNG-3 } & \text { MARROW } \\ 100000.000 & 1.000 E+00 & 1.000 E+00 & 1.000 E+00 & 1.000 E+00 \\ 80000.000 & 1.000 E+00 & 8.500 E-01 & 8.000 E-01 & 1.000 E+00 \\ 70000.000 & 1.000 E+00 & 8.000 E-01 & 5.000 E-01 & 1.000 E+00 \\ 40000.000 & 1.000 E+00 & 7.000 E-01 & 0.000 E+00 & 1.000 E+00 \\ 30000.000 & 1.000 E+00 & 5.000 E-01 & 0.000 E+00 & 1.000 E+00 \\ 25000.000 & 1.000 E+00 & 2.000 E-01 & 0.000 E+00 & 1.000 E+00 \\ 20000.000 & 1.000 E+00 & 8.000 E-02 & 0.000 E+00 & 1.000 E+00 \\ 10000.000 & 6.000 E-01 & 0.000 E+00 & 0.000 E+00 & 1.000 E+00 \\ 8000.000 & 1.000 E-01 & 0.000 E+00 & 0.000 E+00 & 1.000 E+00 \\ 6000.000 & 6.000 E-02 & 0.000 E+00 & 0.000 E+00 & 1.000 E+00 \\ 4000.000 & 3.000 E-02 & 0.000 E+00 & 0.000 E+00 & 1.000 E+00 \\ 3000.000 & 0.000 E+00 & 0.000 E+00 & 0.000 E+00 & 1.000 E+00 \\ 2000.000 & 0.000 E+00 & 0.000 E+00 & 0.000 E+00 & 1.000 E+00 \\ 1000.000 & 0.000 E+00 & 0.000 E+00 & 0.000 E+00 & 1.000 E+00 \\ 800.000 & 0.000 E+00 & 0.000 E+00 & 0.000 E+00 & 9.960 E-01 \\ 700.000 & 0.000 E+00 & 0.000 E+00 & 0.000 E+00 & 9.000 E-01 \\ 600.000 & 0.000 E+00 & 0.000 E+00 & 0.000 E+00 & 4.000 E-01 \\ 500.000 & 0.000 E+00 & 0.000 E+00 & 0.000 E+00 & 5.000 E-02 \\ 400.000 & 0.000 E+00 & 0.000 E+00 & 0.000 E+00 & 0.000 E+00 \\ 300.000 & 0.000 E+00 & 0.000 E+00 & 0.000 E+00 & 0.000 E+00 \\ 100.000 & 0.000 E+00 & 0.000 E+00 & 0.000 E+00 & 0.000 E+00 \\ 75.000 & 0.000 E+00 & 0.000 E+00 & 0.000 E+00 & 0.000 E+00 \\ 50.000 & 0.000 E+00 & 0.000 E+00 & 0.000 E+00 & 0.000 E+00 \\ 30.000 & 0.000 E+00 & 0.000 E+00 & 0.000 E+00 & 0.000 E+00 \\ 15.000 & 0.000 E+00 & 0.000 E+00 & 0.000 E+00 & 0.000 E+00 \\ 5.000 & 0.000 E+00 & 0.000 E+00 & 0.000 E+00 & 0.000 E+00 \\ 1.000 & 0.000 E+00 & 0.000 E+00 & 0.000 E+00 & 0.000 E+00 \\ 0.100 & 0.000 E+00 & 0.000 E+00 & 0.000 E+00 & 0.000 E+00 \\ 0.010 & 0.000 E+00 & 0.000 E+00 & 0.000 E+00 & 0.000 E+00 \\ 0.010 & 0.000 E+00 & 0.000 E+00 & 0.000 E+00 & 0.000 E+00\end{array}$


RUN DATE: [ 16-APR-95 AT 15:56:13]

_INCIDENT-FREE_HAND_CHECK_RAIL_NONLINEAR_

DISPERSAL ACCIDENT INPUT

$\begin{array}{cc}\begin{array}{c}\text { AREADA } \\ \text { (M SQ) }\end{array} & \begin{array}{c}\text { DILUTION } \\ \text { FACTOR* } \\ 4.590 E+02\end{array} \\ 1.530 E+03 & 3.420 E-03 \\ 3.940 E+03 & 1.720 E-03 \\ 1.250 E+04 & 8.580 E-04 \\ 3.040 E+04 & 3.420 E-04 \\ 6.850 E+04 & 1.720 E-04 \\ 1.760 E+05 & 8.580 E-05 \\ 4.450 E+05 & 3.420 E-05 \\ 8.590 E+05 & 1.720 E-05 \\ 2.550 E+06 & 8.580 E-06 \\ 4.450 E+06 & 3.420 E-06 \\ 1.030 E+07 & 1.720 E-06 \\ 2.160 E+07 & 8.580 E-07 \\ 5.520 E+07 & 3.420 E-07 \\ 1.770 E+08 & 1.720 E-07 \\ 4.890 E+08 & 8.580 E-08 \\ 8.120 E+08 & 5.420 E-08 \\ 1.350 E+09 & 4.300 E-08 \\ & 3.420 E-08\end{array}$

* DILUTION FACTOR UNITS ARE (CI-SEC/N**3/CI-RELEASED)

\begin{tabular}{|c|c|c|}
\hline & RADIST(M) & \\
\hline $\begin{array}{l}\text { RURAL } \\
3.050 E+00 \\
6.100 E+00 \\
9.100 E+00 \\
1.220 E+01 \\
1.520 E+01 \\
3.050 E+01 \\
6.100 E+01 \\
9.140 E+01 \\
1.524 E+02 \\
3.050 E+02\end{array}$ & $\begin{array}{l}\text { SUBURBAN } \\
3.050 E+00 \\
6.100 E+00 \\
9.100 E+00 \\
1.220 E+01 \\
1.520 E+01 \\
3.050 E+01 \\
6.100 E+01 \\
9.140 E+01 \\
1.524 E+02 \\
3.050 E+02\end{array}$ & $\begin{array}{l}\text { URBAN } \\
3.050 E+00 \\
6.100 E+00 \\
9.100 E+00 \\
1.220 E+01 \\
1.520 E+01 \\
3.050 E+01 \\
6.100 E+01 \\
9.140 E+01 \\
1.524 E+02 \\
3.050 E+02\end{array}$ \\
\hline
\end{tabular}

BUILDING DOSE FACTOR

FRACTION OF LAND UNDER CULTIVATION

CONTAMINATION CLEAN. UP LEVEL (UCI $/ M^{* *} 2$ )

BREATHING RATE ( $\left.M{ }^{\star \star} 3 / \mathrm{SEC}\right)$
$=8.600 \mathrm{E}-03$

$=4.400 \mathrm{E}-01$

$=1.000 \mathrm{E}+09$

$=3.300 \mathrm{E}-04$ 
RUN DATE: [ 16-APR-95 AT 15:56:13]

_INCIDENT-FREE_HAND_CHECK_RAIL_NONLINEAR_

REGULATORY CHECKS

MODE 2 HAS BEEN REDESIGNATED AS EXCLUSIVE USE

FOR THE SHIPMENT OF

SNF BY MODE 2

THE DOSE RATE AT 2 METERS COULD EXCEED 10 MR/HR

PPS*TI HAS BEEN RESET TO EQUAL 14.00 
RUN DATE: [ 16-APR-95 AT 15:56:13]

_INCIDENT-FREE_HAND_CHECK_RAIL_NONLINEAR_

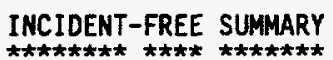

INCIDENT-FREE POPULATION EXPOSURE IN PERSON-REM

PASSENGR CREW HANDLERS OFF LINK ON LINK STOPS STORAGE TOTALS LINK $10.00 E+00$ 1.12E-02 $0.00 E+00 \quad 1.70 E-07 \quad 6.62 E-08 \quad 8.68 E-03 \quad 0.00 E+00 \quad 1.99 E-02$

TOTALS: $0.00 E+00 \quad 1.12 E-02 \quad 0.00 E+00 \quad 1.70 E-07 \quad 6.62 E-08 \quad 8.68 E-03 \quad 0.00 E+00 \quad 1.99 E-02$

MAXIMUM INDIVIDUAL IN-TRANSIT DOSE

LINK $1 \quad 3.34 E-07$ REM 
RUN DATE: [ 16-APR-95 AT 15:56:13 ]

_INCIDENT-FREE_HAND_CHECK_RAIL_NONLINEAR_

INCIDENT-FREE IMPORTANCE ANALYSIS SUMMARY FOR LINK 1

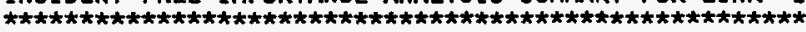

INDEX DESCRIPTION OF PARAMETER

1 DOSE RATE (TRANSPORT INDEX)

2 NUMBER OF SHIPMENTS

3 K ZERO

4 PACKAGES PER SHIPMENT

5 DISTANCE TRAVELED

6 PERSONS EXPOSED WHILE STOPPED

7 FRACTION OF TRAVEL - RURAL

8 POPULATION DENSITY - RURAL

9 NUMBER OF PEOPLE PER VEHICLE

10 TRAFFIC COUNT - RURAL

11 EXPOSURE TIME FOR HANDLERS

12 NUMBER OF CREW MEMBERS

13 NUMBER OF HANDLINGS

DISTANCE FROM SOURCE TO CREW

PERSONS EXPOSED PER HANDLING

HANDLER EXPOSURE DISTANCE

NUMBER OF FLIGHT ATTENDANTS

TRAFFIC COUNT - URBAN

TRAFFIC COUNT - SUBURBAN

FRACTION OF RUSH HOUR TRAVEL

FRACTION OF TRAVEL ON FREEMAYS

STORAGE EXPOSURE DISTANCE

NUMBER OF PERSONS EXPOSED DURING STORAGE

STORAGE TIME PER SHI PMENT

SUBURBAN SHIELDING FACTOR (RS)

VELOCITY - SUBURBAN

POPULATION DENSITY - SUBURBAN

FRACTION OF TRAVEL - SUBURBAN

RATIO OF PEDESTRIAN DENSITY (RPD)

FRACTION OF TRAVEL - URBAN

POPULATION DENSITY - URBAN

STOP TIME

URBAN SHIELDING FACTOR (RU)

FRACTION OF TRAVEL ON CITY STREETS

VELOCITY - URBAN

RURAL SHIELDING FACTOR (RR)

VELOCITY - RURAL

38 EXPOSURE DISTANCE WHILE STOPPED
IMPORTANCE

$1.989 \mathrm{E}-04$

$1.989 E-04$

$1.989 \mathrm{E}-04$

$1.989 E-04$

8.682E-05

8.682E-05

2.358E-09

$1.696 \mathrm{E}-09$

6.620E-10

$6.620 \mathrm{E}-10$

$0.000 E+00$

$0.000 E+00$

$0.000 E+00$

$0.000 E+00$

$0.000 E+00$

$0.000 E+00$

$0.000 E+00$

$0.000 \mathrm{E}+00$

$0.000 E+00$

$0.000 E+00$

$0.000 E+00$

$0.000 E+00$

$0.000 E+00$

$0.000 E+00$

$0.000 E+00$

$0.000 E+00$

$0.000 \mathrm{E}+00$

$0.000 E+00$

$0.000 E+00$

$0.000 E+00$

$0.000 E+00$

$0.000 E+00$

$0.000 E+00$

$0.000 E+00$

$0.000 E+00$

$0.000 E+00$

$-3.020 E-09$

$-1.736 E-04$

THE IMPORTANCE VALUE ESTIMATES THE PERSON-REM INFLUENCE OF A ONE PERCENT INCREASE IN THE PARAMETER

TOTAL EXPOSED POPULATION: INCIDENT-FREE
RAIL RURAL
RAIL SUBURBAN
9. $60 E+00$ PERSONS
RAIL URBAN
$0.00 E+00$ PERSONS
$0.00 E+00$ PERSONS
TOTAL $9.60 E+00$ PERSONS

TOTAL EXPOSED POPULATION: ACCIDENT

(PERSONS UNDER PLUME FOOTPRINT FOR A SINGLE ACCIDENT)

EOI
RAIL RURAL
$0.00 E+00$ PERSONS
RAIL SUBURBAN
$0.00 E+00$ PERSONS
RAIL URBAN
$0.00 E+00$ PERSONS

END OF RUN 


\section{APPENDIX B}

\section{RADTRAN 4 OUTPUT FOR ACCIDENT RISKS}


B-2 


\section{B.1 ACC6.0UT}

\section{RADTRAN4 OUTPUT FOR FIRST CASE}

B-3 
B-4 


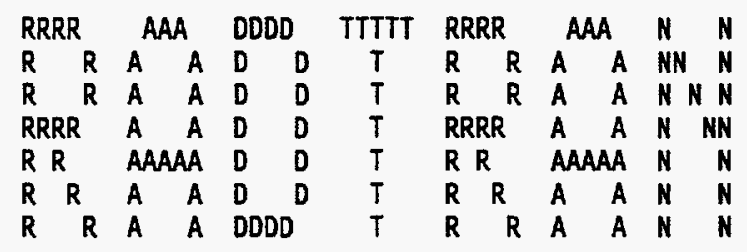

RADTRAN 4.0.17 VERSION DATE: NOVEMBER 8, 1994

\section{MODE DESCRIPTIONS}

$\begin{array}{cll}\text { NUMBER } & \text { NAME } & \text { CHARACTERIZATION } \\ 1 & \text { TRUCK } & \text { LONG HAUL VEHICLE } \\ 2 & \text { RAIL } & \text { COMNERCIAL TRAIN } \\ 3 & \text { BARGE } & \text { INLAND VESSEL } \\ 4 & \text { SHIP } & \text { OPEN SEA VESSEL } \\ 5 & \text { CARGO AIR } & \text { CARGO AIRCRAFT } \\ 6 & \text { PASS AIR } & \text { PASSENGER AIRCRAFT } \\ 7 & \text { P-VAN } & \text { PASSENGER VAN } \\ 8 & \text { CVAN-T } & \text { COMMERCIAL VAN } \\ 9 & \text { CVAN-R } & \text { COMMERCIAL VAN } \\ 10 & \text { CVAN-CA } & \text { COMMERCIAL VAN }\end{array}$


ECHO CHECK

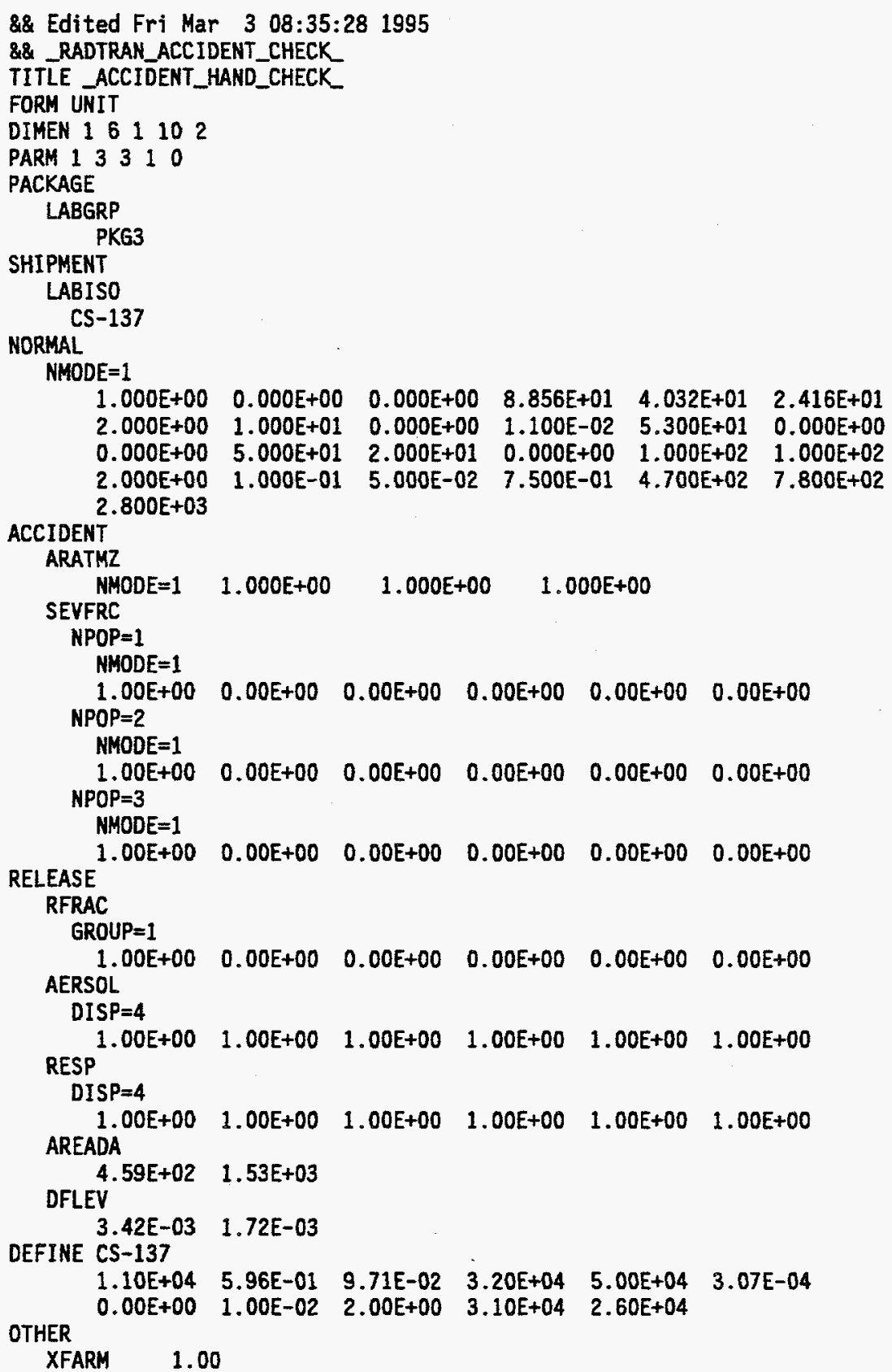

XFARM $\quad 1.00$ 
RUN DATE: [ 3-MAR-95 AT 08:35:48 ] _ACCIDENT_HAND_CHECK_

EOF

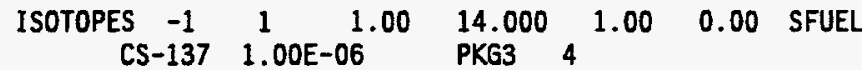
DISTKM

NMODE $=1 \quad 1.00$

PKGSIZ

SFUEL $\quad 5.20$

EOF 
ACCIDENT_HAND_CHECK_

\begin{tabular}{lc}
\multicolumn{1}{c}{ ZONE } & POPULATION DENSITY \\
& (PERSONS PER SQ KM) \\
RURAL & 6. \\
SUBURBAN & 719. \\
URBAN & 3861.
\end{tabular}

PACKAGE CHARACTERISTICS

$\begin{array}{cccc}\text { FOR } & \text { DIMENSION } & \text { EFFECTIVE } & \text { K(0) } \\ \text { MATERIAL } & \text { (METERS) } & \text { DIMENSION } & \text { METERS SQ. } \\ \text { SFUEL } & 5.200 E+00 & 4.677 E+00 & 1.115 E+01\end{array}$

$K(0)$ IS TI TO DOSE RATE CONVERSION FACTOR

PACKAGE HANDLING THRESHOLDS (METERS)

PKESZ1 $=5.000 \mathrm{E}-01$

PKGSZ2 $=1.000 E+00$

PACKAGES .LE. PKGSZI ARE HAND CARRIED

PACKAGES .GT. PKGSZ1 AND .LE. PKGSZ2 ARE HANDLED BY SMALL EQUIPMENT

PACKAGES .GT. PKGSZZ ARE HANDLED BY HEAVY EQUIPMENT

MATERIAL CHARACTERISTICS

$\begin{array}{rrr} & \text { FRACTION } & \text { FRACTION } \\ \text { MATERIAL } & \text { OF GAMMA } & \text { OF NEUTRON } \\ \text { SFUEL } & 1.000 E+00 & 0.000 E+00\end{array}$


RUN DATE: [ 3-MAR-95 AT 08:35:48 ]

PAGE 5

_ACCIDENT_HAND_CHECK_

MODE CHARACTERISTICS

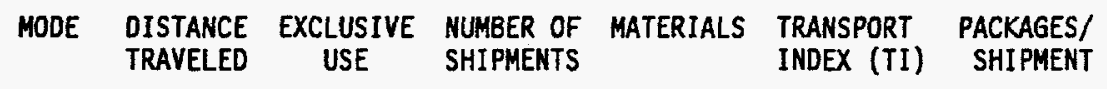

TRUCK $1.00 E+00$ YES $1.00 \mathrm{E}+00$

SFUEL $\quad 1.40 E+01 \quad 1.00 E+00$

BUILDING SHIELDING OPTION= 3

(1=TOTAL SHIELDING, 2=PARTIAL SHIELOING, 3=NO SHIELDING)

$R P D=6.000 E+00$

(RATIO OF PEDESTRIAN DENSITY (PEDESTRIAN/KM SQ OF SIDEWALK)

TO POPULATION DENSITY (PEOPLE/KM SQ IN URBAN AREAS)

$R R=1.000 E+00$

(TRANSMISSION FACTOR FOR RURAL AREAS)

RS $=1.000 E+00$

(TRANSMISSION FACTOR FOR SUBURBAN AREAS)

$R U=1.000 E+00$

(TRANSMISSION FACTOR FOR URBAN AREAS) 
_ACCIDENT_HAND_CHECK_

DNORML INPUT

No

1 FRACTION OF TRAVEL IN RURAL POPULATION ZONE

2 FRACTION OF TRAVEL IN SUBURBAN POPULATION ZONE

3 FRACTION OF TRAVEL IN URBAN POPULATION ZONE

4 VELOCITY IN RURAL POPULATION ZONE (KILOMETERS/HOUR)

5 VELOCITY IN SUBURBAN POP. ZONE 4.032E+01 (KILOMETERS/HOUR)

6 VELOCITY IN URBAN POPULATION $2.416 E+01$ ZONE (KILOMETERS/HOUR)

7 NUMBER OF CREMMEN

$2.000 E+00$

8 DISTANCE FROM SOURCE TO CREW (METERS)

9 NUMBER OF HANOLINGS

1. $000 \mathrm{E}+01$

$0.000 E+00$

10 STOP TIME PER KM (HR/KM)

$1.100 E-02$

11 MINIMUM STOP TIME PER TRIP (HR)

12 ZERO STOP TIME PER TRIP (HR) $0.000 E+00$

13 MINIMUM NUMBER OF RAIL CLASSIF $0.000 E+00$ ICATIONS/INSPECTIONS

14 PERSONS EXPOSED WHILE STOPPED $5.000 E+01$

15 AVERAGE EXPOSURE DISTANCE WHILE STOPPED (METERS)

16 STORAGE TIME PER SHIPMENT (HR)

17 NUMBER OF EXPOSED PERSONS DURING STORAGE

18 AVERAGE EXPOSURE DISTANCE WHILE IN STORAGE (METERS)

19 NUMBER OF PEOPLE PER VEHICLE ON LINK

20 FRACTION OF URBAN TRAVEL DURING RUSH HOUR TRAFFIC

21 FRACTION OF URBAN TRAVEL ON CITY STREETS

22 FRACTION OF RURAL-SUBURBAN TRAVEL ON FREEWAYS

23 *TRAFFIC COUNT PASSING A SPECIFIC POINT-RURAL ZONE

24 *TRAFFIC COUNT PASSING A SPECIFIC POINT-SUBURBAN ZONE

25 *TRAFFIC COUNT PASSING A SPECIFIC POINT-URBAN ZONE

* (ONE WAY VEHICLES/HR)
$2.000 E+01$

$0.000 E+00$

1. $000 \mathrm{E}+02$

$1.000 E+02$

$2.000 \mathrm{E}+00$

5.000E-02

$4.700 \mathrm{E}+02$

$7.800 E+02$

2. $800 E+03$
1.000E-01

7.500E-01 
RUN DATE: [ 3-MAR-95 AT 08:35:48 ]

PAGE

ACCIDENT_HAND_CHECK

ISOTOPE RELATED DATA

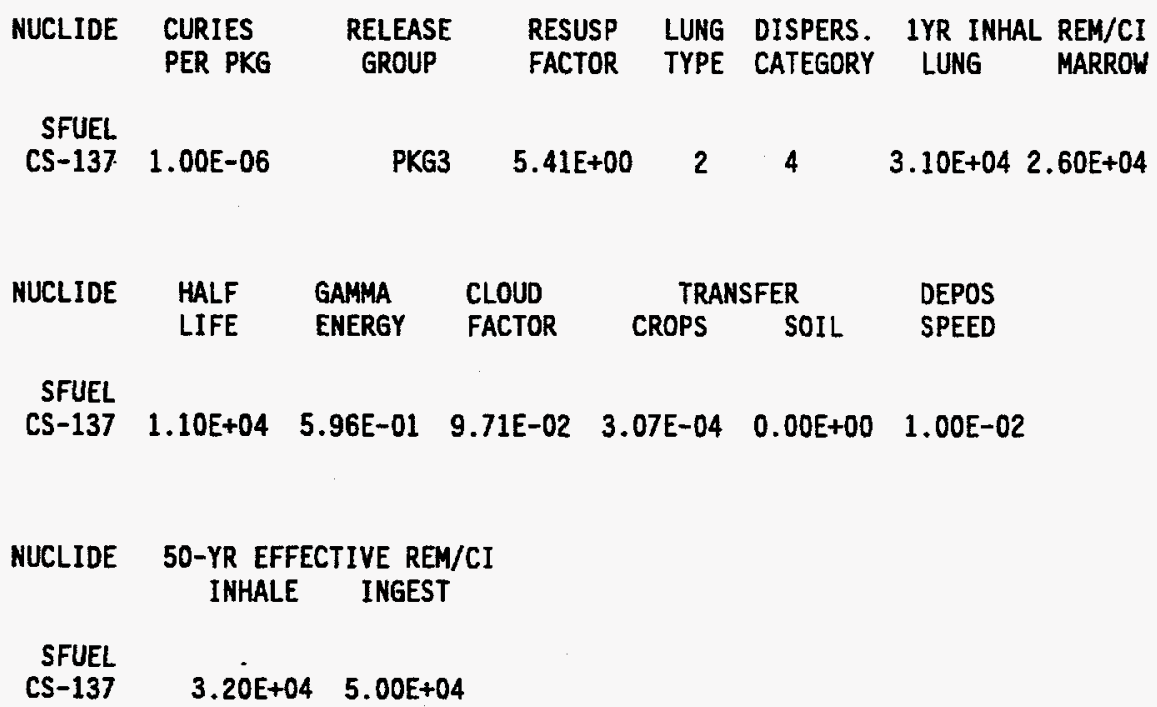


_ACCIDENT_HAND_CHECK_

RELEASE RELATED DATA

\section{ACCIDENT RATES (PER KM)}

$\begin{array}{rrrr}\text { MODE } & \text { RURAL } & \text { SUBURBAN } & \text { URBAN } \\ \text { TRUCK } & 1.000 E+00 & 1.000 E+00 & 1.000 E+00\end{array}$

\section{RELEASE FRACTIONS}

GROUP SEVER: 1 SEVER: 2 SEVER: 3 SEVER: 4 SEVER: 5 SEVER: 6

$1 \quad 1.00 E+00 \quad 0.00 E+00 \quad 0.00 E+00 \quad 0.00 E+00 \quad 0.00 E+00 \quad 0.00 E+00$

\section{ACCIDENT SEVERITY FRACTIONS}

FOR TRUCK

$\begin{array}{cllllll}\text { ZONE } & \text { SEVER: } 1 & \text { SEVER: } 2 & \text { SEVER: } 3 & \text { SEVER: } 4 & \text { SEVER: } 5 & \text { SEVER: } 6 \\ 1 & 1.00 E+00 & 0.00 E+00 & 0.00 E+00 & 0.00 E+00 & 0.00 E+00 & 0.00 E+00 \\ 2 & 1.00 E+00 & 0.00 E+00 & 0.00 E+00 & 0.00 E+00 & 0.00 E+00 & 0.00 E+00 \\ 3 & 1.00 E+00 & 0.00 E+00 & 0.00 E+00 & 0.00 E+00 & 0.00 E+00 & 0.00 E+00\end{array}$


_ACCIDENT_HAND_CHECK_

AEROSOLIZED FRACTION OF RELEASED MATERIAL

$\begin{array}{cllllll}\text { DISP CAT } & \text { SEVER: } 1 & \text { SEVER: } 2 & \text { SEVER: } 3 & \text { SEVER: } 4 & \text { SEVER: } 5 & \text { SEVER: } 6 \\ 1 & 0.00 E+00 & 0.00 E+00 & 0.00 E+00 & 0.00 E+00 & 0.00 E+00 & 0.00 E+00 \\ 2 & 1.00 E-06 & 1.00 E-06 & 1.00 E-06 & 1.00 E-06 & 1.00 E-06 & 1.00 E-06 \\ 3 & 1.00 E-02 & 1.00 E-02 & 1.00 E-02 & 1.00 E-02 & 1.00 E-02 & 1.00 E-02 \\ 4 & 1.00 E+00 & 1.00 E+00 & 1.00 E+00 & 1.00 E+00 & 1.00 E+00 & 1.00 E+00 \\ 5 & 1.00 E-01 & 1.00 E-01 & 1.00 E-01 & 1.00 E-01 & 1.00 E-01 & 1.00 E-01 \\ 6 & 1.00 E+00 & 1.00 E+00 & 1.00 E+00 & 1.00 E+00 & 1.00 E+00 & 1.00 E+00 \\ 7 & 1.00 E+00 & 1.00 E+00 & 1.00 E+00 & 1.00 E+00 & 1.00 E+00 & 1.00 E+00 \\ 8 & 1.00 E+00 & 1.00 E+00 & 1.00 E+00 & 1.00 E+00 & 1.00 E+00 & 1.00 E+00 \\ 9 & 1.00 E+00 & 1.00 E+00 & 1.00 E+00 & 1.00 E+00 & 1.00 E+00 & 1.00 E+00 \\ 10 & 1.00 E+00 & 1.00 E+00 & 1.00 E+00 & 1.00 E+00 & 1.00 E+00 & 1.00 E+00 \\ 11 & 1.00 E+00 & 1.00 E+00 & 1.00 E+00 & 1.00 E+00 & 1.00 E+00 & 1.00 E+00\end{array}$

FRACTION OF AEROSOLS BELOW 10 MICRONS AED

$\begin{array}{cllllll}\text { DISP CAT } & \text { SEVER: } 1 & \text { SEVER: } 2 & \text { SEVER: } 3 & \text { SEVER: } 4 & \text { SEVER: } 5 & \text { SEVER: } 6 \\ 1 & 0.00 E+00 & 0.00 E+00 & 0.00 E+00 & 0.00 E+00 & 0.00 E+00 & 0.00 E+00 \\ 2 & 5.00 E-02 & 5.00 E-02 & 5.00 E-02 & 5.00 E-02 & 5.00 E-02 & 5.00 E-02 \\ 3 & 5.00 E-02 & 5.00 E-02 & 5.00 E-02 & 5.00 E-02 & 5.00 E-02 & 5.00 E-02 \\ 4 & 1.00 E+00 & 1.00 E+00 & 1.00 E+00 & 1.00 E+00 & 1.00 E+00 & 1.00 E+00 \\ 5 & 5.00 E-02 & 5.00 E-02 & 5.00 E-02 & 5.00 E-02 & 5.00 E-02 & 5.00 E-02 \\ 6 & 5.00 E-02 & 5.00 E-02 & 5.00 E-02 & 5.00 E-02 & 5.00 E-02 & 5.00 E-02 \\ 7 & 1.00 E+00 & 1.00 E+00 & 1.00 E+00 & 1.00 E+00 & 1.00 E+00 & 1.00 E+00 \\ 8 & 1.00 E+00 & 1.00 E+00 & 1.00 E+00 & 1.00 E+00 & 1.00 E+00 & 1.00 E+00 \\ 9 & 1.00 E+00 & 1.00 E+00 & 1.00 E+00 & 1.00 E+00 & 1.00 E+00 & 1.00 E+00 \\ 10 & 1.00 E+00 & 1.00 E+00 & 1.00 E+00 & 1.00 E+00 & 1.00 E+00 & 1.00 E+00 \\ 11 & 1.00 E+00 & 1.00 E+00 & 1.00 E+00 & 1.00 E+00 & 1.00 E+00 & 1.00 E+00\end{array}$


RUN DATE: [ 3-MAR-95 AT 08:35:48 ]

ACCIDENT_HAND_CHECK_

COST RELATED DATA

EMERGENCY RESPONSE COST

SEVER: 1 SEVER: 2 SEVER: 3 SEVER: 4 SEVER: 5 SEVER: 6

$10.00 E+00 \quad 0.00 E+00 \quad 0.00 E+00 \quad 0.00 E+00 \quad 0.00 E+00 \quad 0.00 E+00$

ON-SCENE COSTS

(RF=RELEASE FRACTION)

$R F=0 . \quad 0.0 .<R F<=.01 \quad 0.01<R F<=0.10 . .1<R F<=1,0$. 
RUN DATE: [ 3-MAR-95 AT 08:35:48 ]

_ACCIDENT_HAND_CHECK_

HEALTH RELATED DATA

\section{EARLY FATALITY PROBABILITIES}

$\begin{array}{rrrrr}\text { DOSE (REM) } & \text { LUNG-1 } & \text { LUNG }-2 & \text { LUNG-3 } & \text { MARROW } \\ 100000.000 & 1.000 E+00 & 1.000 E+00 & 1.000 E+00 & 1.000 E+00 \\ 80000.000 & 1.000 E+00 & 8.500 E-01 & 8.000 E-01 & 1.000 E+00 \\ 70000.000 & 1.000 E+00 & 8.000 E-01 & 5.000 E-01 & 1.000 E+00 \\ 40000.000 & 1.000 E+00 & 7.000 E-01 & 0.000 E+00 & 1.000 E+00 \\ 30000.000 & 1.000 E+00 & 5.000 E-01 & 0.000 E+00 & 1.000 E+00 \\ 25000.000 & 1.000 E+00 & 2.000 E-01 & 0.000 E+00 & 1.000 E+00 \\ 20000.000 & 1.000 E+00 & 8.000 E-02 & 0.000 E+00 & 1.000 E+00 \\ 10000.000 & 6.000 E-01 & 0.000 E+00 & 0.000 E+00 & 1.000 E+00 \\ 8000.000 & 1.000 E-01 & 0.000 E+00 & 0.000 E+00 & 1.000 E+00 \\ 6000.000 & 6.000 E-02 & 0.000 E+00 & 0.000 E+00 & 1.000 E+00 \\ 4000.000 & 3.000 E-02 & 0.000 E+00 & 0.000 E+00 & 1.000 E+00 \\ 3000.000 & 0.000 E+00 & 0.000 E+00 & 0.000 E+00 & 1.000 E+00 \\ 2000.000 & 0.000 E+00 & 0.000 E+00 & 0.000 E+00 & 1.000 E+00 \\ 1000.000 & 0.000 E+00 & 0.000 E+00 & 0.000 E+00 & 1.000 E+00 \\ 800.000 & 0.000 E+00 & 0.000 E+00 & 0.000 E+00 & 9.960 E-01 \\ 700.000 & 0.000 E+00 & 0.000 E+00 & 0.000 E+00 & 9.000 E-01 \\ 600.000 & 0.000 E+00 & 0.000 E+00 & 0.000 E+00 & 4.000 E-01 \\ 500.000 & 0.000 E+00 & 0.000 E+00 & 0.000 E+00 & 5.000 E-02 \\ 400.000 & 0.000 E+00 & 0.000 E+00 & 0.000 E+00 & 0.000 E+00 \\ 300.000 & 0.000 E+00 & 0.000 E+00 & 0.000 E+00 & 0.000 E+00 \\ 100.000 & 0.000 E+00 & 0.000 E+00 & 0.000 E+00 & 0.000 E+00 \\ 75.000 & 0.000 E+00 & 0.000 E+00 & 0.000 E+00 & 0.000 E+00 \\ 50.000 & 0.000 E+00 & 0.000 E+00 & 0.000 E+00 & 0.000 E+00 \\ 30.000 & 0.000 E+00 & 0.000 E+00 & 0.000 E+00 & 0.000 E+00 \\ 15.000 & 0.000 E+00 & 0.000 E+00 & 0.000 E+00 & 0.000 E+00 \\ 5.000 & 0.000 E+00 & 0.000 E+00 & 0.000 E+00 & 0.000 E+00 \\ 1.000 & 0.000 E+00 & 0.000 E+00 & 0.000 E+00 & 0.000 E+00 \\ 0.100 & 0.000 E+00 & 0.000 E+00 & 0.000 E+00 & 0.000 E+00 \\ 0.010 & 0.000 E+00 & 0.000 E+00 & 0.000 E+00 & 0.000 E+00 \\ 0.010 & 0.000 E+00 & 0.000 E+00 & 0.000 E+00 & 0.000 E+00\end{array}$


RUN DATE: [ 3-MAR-95 AT 08:35:48 ]

_ACCIDENT_HAND_CHECK_

DISPERSAL ACCIDENT INPUT

$\begin{array}{cc}\begin{array}{c}\text { AREADA } \\ \text { (M SQ) }\end{array} & \begin{array}{c}\text { DILUTION } \\ \text { FACTOR* }\end{array} \\ 4.590 E+02 & 3.420 E-03 \\ 1.530 E+03 & 1.720 E-03\end{array}$

* DILUTION FACTOR UNITS ARE (CI-SEC/M**3/CI-RELEASED)

NON-DISPERSAL ACCIDENT INPUT

$\begin{array}{lll} & \text { RADIST(M) } & \\ \text { RURAL } & \text { SUBURBAN } & \text { URBAN } \\ 3.050 E+00 & 3.050 E+00 & 3.050 E+00 \\ 6.100 E+00 & 6.100 E+00 & 6.100 E+00 \\ 9.100 E+00 & 9.100 E+00 & 9.100 E+00 \\ 1.220 E+01 & 1.220 E+01 & 1.220 E+01 \\ 1.520 E+01 & 1.520 E+01 & 1.520 E+01 \\ 3.050 E+01 & 3.050 E+01 & 3.050 E+01 \\ 6.100 E+01 & 6.100 E+01 & 6.100 E+01 \\ 9.140 E+01 & 9.140 E+01 & 9.140 E+01 \\ 1.524 E+02 & 1.524 E+02 & 1.524 E+02 \\ 3.050 E+02 & 3.050 E+02 & 3.050 E+02\end{array}$

BUILDING DOSE FACTOR

FRACTION OF LAND UNDER CULTIVATION

CONTAMINATION CLEAN UP LEVEL (UCI/M**2)

BREATHING RATE (M**3/SEC)
$=8.600 E-03$

$=1.000 E+00$

$=2.000 E-01$

$=3.300 E-04$ 
_ACCIDENT_HAND_CHECK_

\section{REGULATORY CHECKS}

FOR THE SHIPMENT OF SFUEL BY MODE 1

THE DOSE RATE AT 2 METERS COULD EXCEED $10 \mathrm{MR} / \mathrm{HR}$

PPS*TI HAS BEEN RESET TO EQUAL 13.00 
RUN DATE: [ 3-MAR-95 AT 08:35:48] ACCIDENT_HAND_CHECK_ 
RUN DATE: [ 3-MAR-95 AT 08:35:48 ]

_ACCIDENT_HAND_CHECK_

MODE TRUCK

1-YEAR LUNG DOSE - INHALATION PATHWAY

$B D F=1 \quad$ (REM)

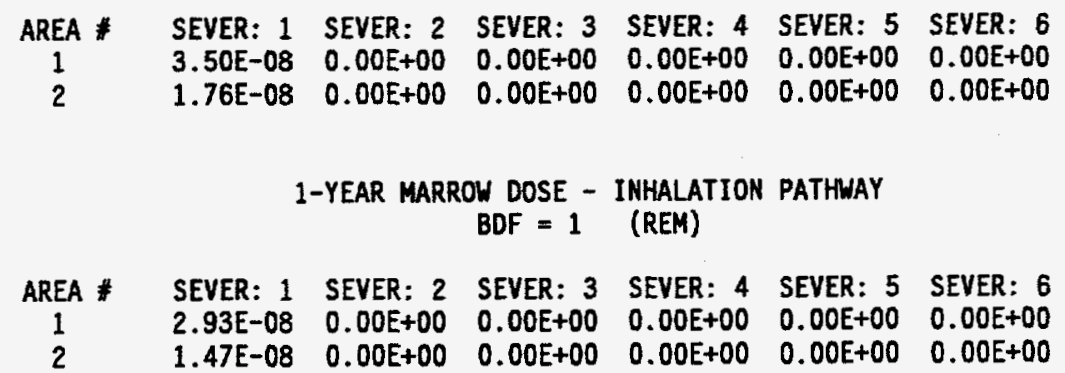


RUN DATE: [ 3-MAR-95 AT 08:35:48 ]

_ACCIDENT_HAND_CHECK_

MODE TRUCK

GROUND SURFACE CONTAMINATION TABLE (MICRO CI/M**2)

BEFORE CLEANUP

AREA * SEVER: 1 SEVER: 2 SEVER: 3 SEVER: 4 SEVER: 5 SEVER: 6

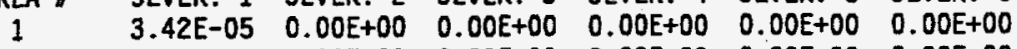

$2 \quad \begin{array}{lllllll}1.72 E-05 & 0.00 E+00 & 0.00 E+00 & 0.00 E+00 & 0.00 E+00 & 0.00 E+00\end{array}$ 
RUN DATE: [ 3-MAR-95 AT 08:35:48 ]

PAGE 17

ACCIDENT_HAND_CHECK_

\section{INCIDENT-FREE SUMMARY

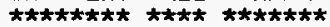

INCIDENT-FREE POPULATION EXPOSURE IN PERSON-REM

PASSENGR CREW HANDLERS OFF LINK ON LINK STOPS STORAGE TOTALS LINK $10.00 E+00$ 3.55E-05 $0.00 E+00 \quad 2.06 E-07 \quad 1.19 E-05 \quad 9.60 E-01 \quad 0.00 E+00 \quad 9.60 E-01$

TOTALS: $0.00 E+00 \quad 3.55 E-05 \quad 0.00 E+00 \quad 2.06 E-07 \quad 1.19 E-05 \quad 9.60 E-01 \quad 0.00 E+00 \quad 9.60 E-01$

MAXIMUM INDIVIDUAL IN-TRANSIT DOSE

LINK 1 5.96E-07 REM 
ACCIDENT_HAND_CHECK

INCIDENT-FREE IMPORTANCE ANALYSIS SUMMARY FOR LINK 1

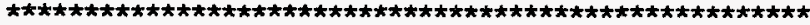

INDEX

1 DOSE RATE (TRANSPORT INDEX)

2 K ZERO

3 NUMBER OF SHIPMENTS

4 PACKAGES PER SHIPMENT

5 DISTANCE TRAVELED

6 PERSONS EXPOSED NHILE STOPPED

7 STOP TIME

8 FRACTION OF TRAVEL - RURAL

9 NUMBER OF CREW MEMBERS

10 NUMBER OF PEOPLE PER VEHICLE

11 TRAFFIC COUNT - RURAL

12 POPULATION DENSITY - RURAL

13 FRACTION OF RUSH HOUR TRAVEL

14 STORAGE EXPOSURE DISTANCE

15 NUMBER OF HANDLINGS

16 EXPOSURE TIME FOR HANDLERS

17 PERSONS EXPOSED PER HANDLING

18 NUMBER OF FLIGHT ATTENDANTS

19 TRAFFIC COUNT - URBAN

20 TRAFFIC COUNT - SUBURBAN

21 HANDLER EXPOSURE DISTANCE

22 NUMBER OF PERSONS EXPOSED DURING STORAGE

23 STORAGE TIME PER SHIPMENT

24 SUBURBAN SHIELDING FACTOR (RS)

25 VELOCITY - SUBURBAN

26 POPULATION DENSITY - SUBURBAN

27 FRACTION OF TRAVEL - SUBURBAN

28 RATIO OF PEDESTRIAN DENSITY (RPD)

29 FRACTION OF TRAVEL - URBAN

30 POPULATION DENSITY - URBAN

31 URBAN SHIELDING FACTOR (RU)

32 FRACTION OF TRAVEL ON CITY STREETS

33 RURAL SHIELDING FACTOR (RR)

34 VELOCITY - URBAN

35 FRACTION OF TRAVEL ON FREEWAYS

36 VELOCITY - RURAL

37 DISTANCE FROM SOURCE TO CREW

38 EXPOSURE DISTANCE WHILE STOPPED
IMPORTANCE

$9.596 E-03$

$9.596 \mathrm{E}-03$

$9.596 \mathrm{E}-03$

$9.596 \mathrm{E}-03$

$9.596 \mathrm{E}-03$

$9.596 \mathrm{E}-03$

9.596E-03

4.767E-07

3.552E-07

$1.194 E-07$

$1.194 \mathrm{E}-07$

2.057E-09

$9.237 \mathrm{E}-16$

$0.000 E+00$

$0.000 E+00$

$0.000 E+00$

$0.000 E+00$

$0.000 E+00$

$0.000 E+00$

$0.000 E+00$

$0.000 E+00$

$0.000 E+00$

$0.000 E+00$

$0.000 E+00$

$0.000 E+00$

$0.000 E+00$

$0.000 E+00$

$0.000 E+00$

$0.000 E+00$

$0.000 E+00$

$0.000 E+00$

$0.000 E+00$

$0.000 E+00$

$0.000 E+00$

$-1.089 E-07$

$-5.961 E-07$

$-6.594 E-07$

$-1.919 E-02$

THE IMPORTANCE VALUE ESTIMATES THE PERSON-REM INFLUENCE OF A ONE PERCENT INCREASE IN THE PARAMETER 
_ACCIDENT_HAND_CHECK_

\section{ACCIDENT SUMMARY

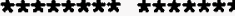

NUMBER OF EXPECTED ACCIDENTS -- MODE TRUCK

$\begin{array}{clll}\text { CATEGORY } & \text { RURAL } & \text { SUBURB } & \text { URBAN } \\ 1 & 1.00 E+00 & 0.00 E+00 & 0.00 E+00 \\ 2 & 0.00 E+00 & 0.00 E+00 & 0.00 E+00 \\ 3 & 0.00 E+00 & 0.00 E+00 & 0.00 E+00 \\ 4 & 0.00 E+00 & 0.00 E+00 & 0.00 E+00 \\ 5 & 0.00 E+00 & 0.00 E+00 & 0.00 E+00 \\ 6 & 0.00 E+00 & 0.00 E+00 & 0.00 E+00\end{array}$

EARLY. FATALITY CONSEQUENCES -- MODE TRUCK

$\begin{array}{clll}\text { CATEGORY } & \text { RURAL } & \text { SUBURB } & \text { URBAN } \\ 1 & 0.00 E+00 & 0.00 E+00 & 0.00 E+00 \\ 2 & 0.00 E+00 & 0.00 E+00 & 0.00 E+00 \\ 3 & 0.00 E+00 & 0.00 E+00 & 0.00 E+00 \\ 4 & 0.00 E+00 & 0.00 E+00 & 0.00 E+00 \\ 5 & 0.00 E+00 & 0.00 E+00 & 0.00 E+00 \\ 6 & 0.00 E+00 & 0.00 E+00 & 0.00 E+00\end{array}$

ECONOMIC CONSEQUENCES -- MODE TRUCK

$\begin{array}{clll}\text { CATEGORY } & \text { RURAL } & \text { SUBURB } & \text { URBAN } \\ 1 & 0.00 E+00 & 0.00 E+00 & 0.00 E+00 \\ 2 & 0.00 E+00 & 0.00 E+00 & 0.00 E+00 \\ 3 & 0.00 E+00 & 0.00 E+00 & 0.00 E+00 \\ 4 & 0.00 E+00 & 0.00 E+00 & 0.00 E+00 \\ 5 & 0.00 E+00 & 0.00 E+00 & 0.00 E+00 \\ 6 & 0.00 E+00 & 0.00 E+00 & 0.00 E+00\end{array}$

RADIOLOGICAL CONSEQUENCES -- MODE TRUCK 50 YEAR POPULATION DOSE IN PERSON REM

$\begin{array}{clll}\text { CATEGORY } & \text { RURAL } & \text { SUBURB } & \text { URBAN } \\ 1 & 6.60 E-07 & 1.64 E-05 & 5.32 E-05 \\ 2 & 0.00 E+00 & 0.00 E+00 & 0.00 E+00 \\ 3 & 0.00 E+00 & 0.00 E+00 & 0.00 E+00 \\ 4 & 0.00 E+00 & 0.00 E+00 & 0.00 E+00 \\ 5 & 0.00 E+00 & 0.00 E+00 & 0.00 E+00 \\ 6 & 0.00 E+00 & 0.00 E+00 & 0.00 E+00\end{array}$


RUN DATE: [ 3-MAR-95 AT 08:35:48]

_ACCIDENT_HAND_CHECK_

EXPECTED VALUES OF POPULATION RISK IN PERSON REM

$\begin{array}{rllllll} & \text { GROUND } & \text { INHALED } & \text { RESUSPD } & \text { CLOUDSH } & \text { *INGESTION TOTAL } \\ \text { SFUEL } & & & & & & \\ \text { CS-137 } & 1.35 E-07 & 2.84 E-10 & 1.25 E-09 & 2.61 E-12 & 5.23 E-07 & 6.60 E-07 \\ \text { TOTALS: } & 1.35 E-07 & 2.84 E-10 & 1.25 E-09 & 2.61 E-12 & 5.23 E-07 & 6.60 E-07\end{array}$

* NOTE THAT INGESTION RISK IS A SOCIETAL RISK;

THE USER MAY WISH TO TREAT THIS VALUE SEPARATELY. 
RUN DATE: [ 3-MAR-95 AT 08:35:48 ]

_ACCIDENT_HAND_CHECK

EXPECTED RISK VALUES - OTHER

\begin{tabular}{|c|c|c|}
\hline LINK & $\begin{array}{c}E C O N \\
\$ \$ \\
0.00 E+00\end{array}$ & $\begin{array}{l}\text { EARLY } \\
\text { FATALITY } \\
0.00 E+00\end{array}$ \\
\hline TOTAI & $0.00 E+00$ & $0.00 E+00$ \\
\hline
\end{tabular}

TOTAL EXPOSED POPULATION: INCIDENT-FREE
TRUCK RURAL
TRUCK SUBURBAN
9.60E+00 PERSONS
$0.00 E+00$ PERSONS
TRUCK URBAN
$0.00 E+00$ PERSONS
TOTAL 9.60E+00 PERSONS

TOTAL EXPOSED POPULATION: ACCIDENT

(PERSONS UNDER PLUME FOOTPRINT FOR A SINGLE ACCIDENT)

TRUCK RURAL

TRUCK SUBURBAN

TRUCK URBAN
9.18E-03 PERSONS

$1.10 E+00$ PERSONS

5.91E+00 PERSONS

EOI

END OF RUN 
B-26 


\section{B.2 RISK2.OUT}

RADTRAN 4 OUTPUT FOR SECOND CASE 
B-28 


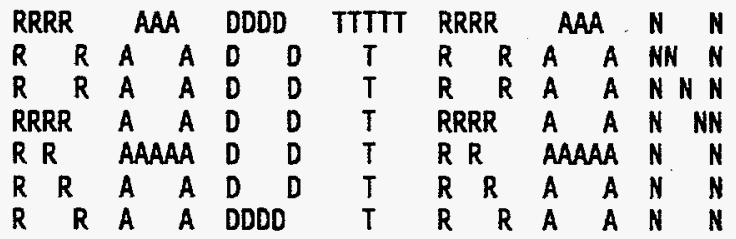

RADTRAN 4.0.17 VERSION DATE: NOVEMBER 8, 1994

MODE DESCRIPTIONS

$\begin{array}{cll}\text { NUMBER } & \text { NAME } & \text { CHARACTERIZATION } \\ 1 & \text { TRUCK } & \text { LONG HAUL VEHICLE } \\ 2 & \text { RAIL } & \text { COMMERCIAL TRAIN } \\ 3 & \text { BARGE } & \text { INLAND VESSEL } \\ 4 & \text { SHIP } & \text { OPEN SEA VESSEL } \\ 5 & \text { CARGO AIR } & \text { CARGO AIRCRAFT } \\ 6 & \text { PASS AIR } & \text { PASSENGER AIRCRAFT } \\ 7 & \text { P-VAN } & \text { PASSENGER VAN } \\ 8 & \text { CVAN-T } & \text { COMMERCIAL VAN } \\ 9 & \text { CVAN-R } & \text { COMMERCIAL VAN } \\ 10 & \text { CVAN-CA } & \text { COMMERCIAL VAN }\end{array}$


ECHO CHECK

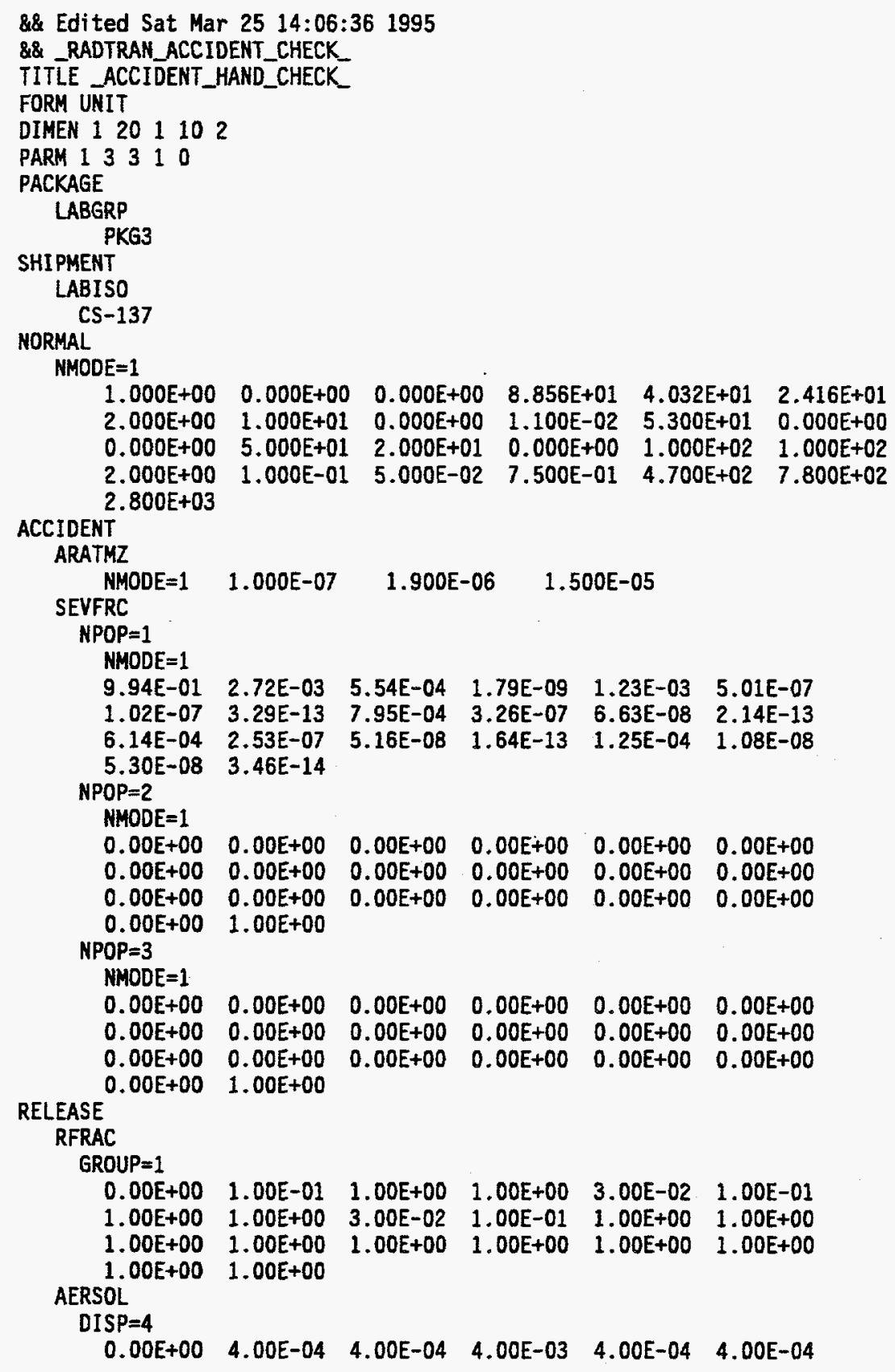


_ACCIDENT_HAND_CHECK_

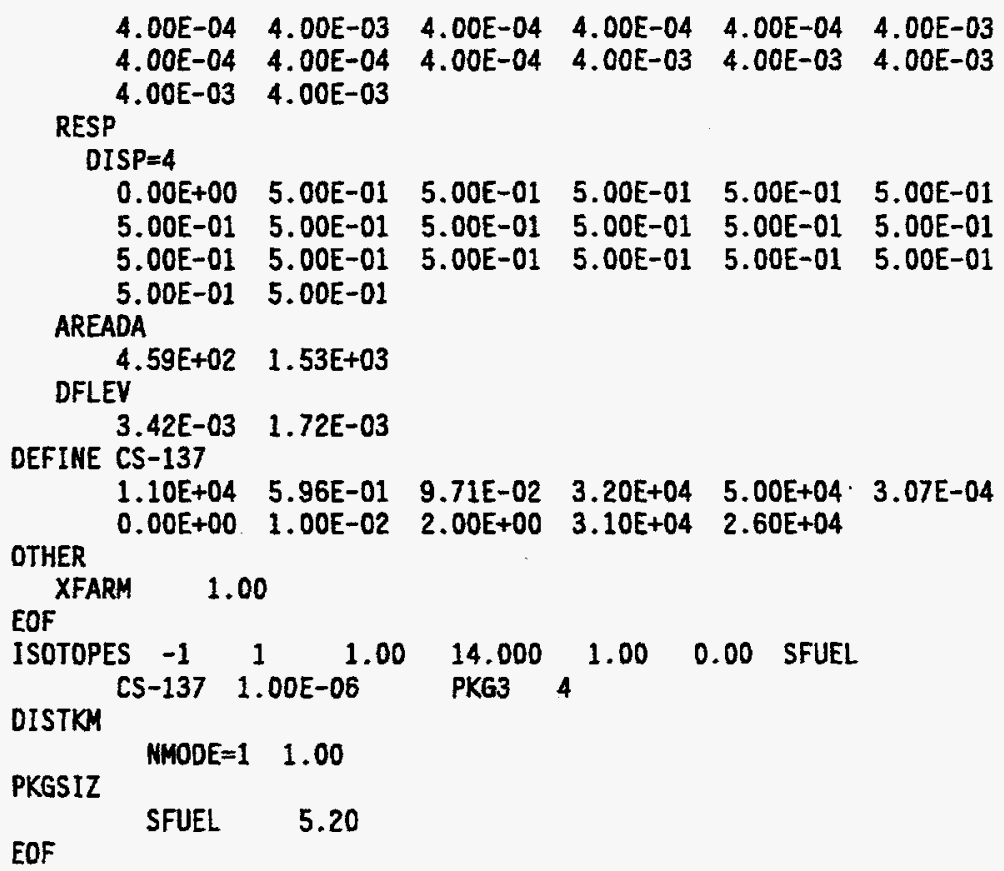


_ACCIDENT_HAND_CHECK_

\begin{tabular}{lc}
\multicolumn{1}{c}{ ZONE } & $\begin{array}{c}\text { POPULATION DENSITY } \\
\text { (PERSONS PER SQ KN) }\end{array}$ \\
RURAL & 6. \\
SUBURBAN & 719. \\
URBAN & 3861.
\end{tabular}

PACKAGE CHARACTERISTICS

$\begin{array}{cccc}\text { FOR } & \text { DIMENSION } & \text { EFFECTIVE } & \text { K(0) } \\ \text { MATERIAL } & \text { (METERS) } & \text { DIMENSION } & \text { METERS SQ. } \\ \text { SFUEL } & 5.200 E+00 & 4.677 E+00 & 1.115 E+01\end{array}$

$K(0)$ IS TI TO DOSE RATE CONVERSION FACTOR

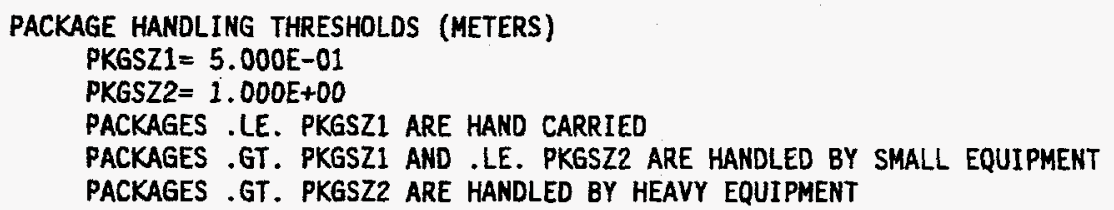

MATERIAL CHARACTERISTICS

$\begin{array}{rrr} & \text { FRACTION } & \text { FRACTION } \\ \text { MATERIAL } & \text { OF GAMMA } & \text { OF NEUTRON } \\ \text { SFUEL } & 1.000 E+00 & 0.000 E+00\end{array}$


RUN DATE: [ 25-MAR-95 AT 14:06:57]

ACCIDENT_HAND_CHECK_

MODE CHARACTERISTICS

$\begin{array}{ccccccc}\text { MODE } & \begin{array}{c}\text { DISTANCE } \\ \text { TRAVELED }\end{array} & \begin{array}{c}\text { EXCLUSIVE } \\ \text { USE }\end{array} & \begin{array}{c}\text { NUMBER OF } \\ \text { SHIPMENTS }\end{array} & \text { MATERIALS } & \begin{array}{c}\text { TRANSPORT } \\ \text { INDEX (TI) }\end{array} & \begin{array}{c}\text { PACKAGES/ } \\ \text { SHIPMENT }\end{array} \\ \text { TRUCK } & 1.00 E+00 & \text { YES } & 1.00 E+00 & & & \\ & & & & \text { SFUEL } & 1.40 E+01 & 1.00 E+00\end{array}$

BUILDING SHIELDING OPTION $=3$

(1=TOTAL SHIELDING, 2=PARTIAL SHIELDING, 3=NO SHIELDING)

$R P D=6.000 E+00$

(RATIO OF PEDESTRIAN DENSITY (PEDESTRIAN/KM SQ OF SIDEWALK)

TO POPULATION DENSITY (PEOPLE/KM SQ IN URBAN AREAS)

$R R=1.000 E+00$

(TRANSMISSION FACTOR FOR RURAL AREAS)

$R S=1.000 E+00$

(TRANSMISSION FACTOR FOR SUBURBAN AREAS)

$R U=1.000 E+00$

(TRANSMISSION FACTOR FOR URBAN AREAS) 
_ACCIOENT_HANO_CHECK_

DNORML INPUT

\begin{tabular}{|c|c|c|}
\hline 10 & & TRUCK \\
\hline & $\begin{array}{l}\text { FRACTION OF TRAVEL } \\
\text { IN RURAL POPULATION ZONE }\end{array}$ & $1.000 E+00$ \\
\hline 2 & $\begin{array}{l}\text { FRACTION OF TRAVEL } \\
\text { IN SUBURBAN POPULATION ZONE }\end{array}$ & $0.000 E+00$ \\
\hline & $\begin{array}{l}\text { FRACTION OF TRAVEL } \\
\text { IN URBAN POPULATION ZONE }\end{array}$ & $0.000 E+00$ \\
\hline & $\begin{array}{l}\text { VELOCITY IN RURAL POPULATION } \\
\text { ZONE (KILOMETERS/HOUR) }\end{array}$ & $8.856 E+01$ \\
\hline & $\begin{array}{l}\text { VELOCITY IN SUBURBAN POP. ZONE } \\
\text { (KILOMETERS/HOUR) }\end{array}$ & $4.032 E+01$ \\
\hline & $\begin{array}{l}\text { VELOCITY IN URBAN POPULATION } \\
\text { ZONE (KILOMETERS/HOUR) }\end{array}$ & $2.416 \mathrm{E}+01$ \\
\hline & NUMBER OF CREMMEN & $2.000 E+00$ \\
\hline & $\begin{array}{l}\text { DISTANCE FROM SOURCE TO CREW } \\
\text { (METERS) }\end{array}$ & $1.000 E+01$ \\
\hline & NUMBER OF HANDLINGS & $0.000 E+00$ \\
\hline & STOP TIME PER KM (HR/KM) & $1.100 E-02$ \\
\hline & $\begin{array}{l}\text { MINIMUM STOP TIME PER TRIP } \\
(H R)\end{array}$ & $5.300 E+01$ \\
\hline & ZERO STOP TIME PER TRIP (HR) & $0.000 E+00$ \\
\hline & $\begin{array}{l}\text { MINIMUM NUMBER OF RAIL CLASSIF } \\
\text { ICATIONS/INSPECTIONS }\end{array}$ & $0.000 E+00$ \\
\hline & PERSONS EXPOSED WHILE STOPPED & $5.000 E+01$ \\
\hline & $\begin{array}{l}\text { AVERAGE EXPOSURE DISTANCE } \\
\text { WHILE STOPPED (METERS) }\end{array}$ & $2.000 E+01$ \\
\hline & $\begin{array}{l}\text { STORAGE TIME PER SHIPMENT } \\
\text { (HR) }\end{array}$ & $0.000 E+00$ \\
\hline & $\begin{array}{l}\text { NUMBER OF EXPOSED PERSONS } \\
\text { DURING STORAGE }\end{array}$ & $1.000 E+02$ \\
\hline & $\begin{array}{l}\text { AVERAGE EXPOSURE DISTANCE } \\
\text { WHILE IN STORAGE (METERS) }\end{array}$ & $1.000 E+02$ \\
\hline & $\begin{array}{l}\text { NLMBER OF PEOPLE PER VEHICLE } \\
\text { ON LINK }\end{array}$ & $2.000 E+00$ \\
\hline & $\begin{array}{l}\text { FRACTION OF URBAN TRAVEL } \\
\text { DURING RUSH HOUR TRAFFIC }\end{array}$ & $1: 000 E-01$ \\
\hline & $\begin{array}{l}\text { FRACTION OF URBAN TRAVEL } \\
\text { ON CITY STREETS }\end{array}$ & $5.000 E-02$ \\
\hline & $\begin{array}{l}\text { FRACTIION OF RURAL-SUBURBAN } \\
\text { TRAVEL ON FREEWAYS }\end{array}$ & $7.500 E-01$ \\
\hline & $\begin{array}{l}\text { *TRAFFIC COUNT PASSING A } \\
\text { SPECIFIC POINT-RURAL ZONE }\end{array}$ & $4.700 E+02$ \\
\hline & $\begin{array}{l}\text { *TRAFFIC COUNT PASSING A } \\
\text { SPECIFIC POINT-SUBURBAN ZONE }\end{array}$ & $7.800 E+02$ \\
\hline & $\begin{array}{l}\text { *TRAFFIC COUNT PASSING A } \\
\text { SPECIFIC POINT-URBAN ZONE }\end{array}$ & \\
\hline
\end{tabular}


RUN DATE: [ 25-MAR-95 AT 14:06:57]

_ACCIDENT_HAND_CHECK_

ISOTOPE RELATED DATA

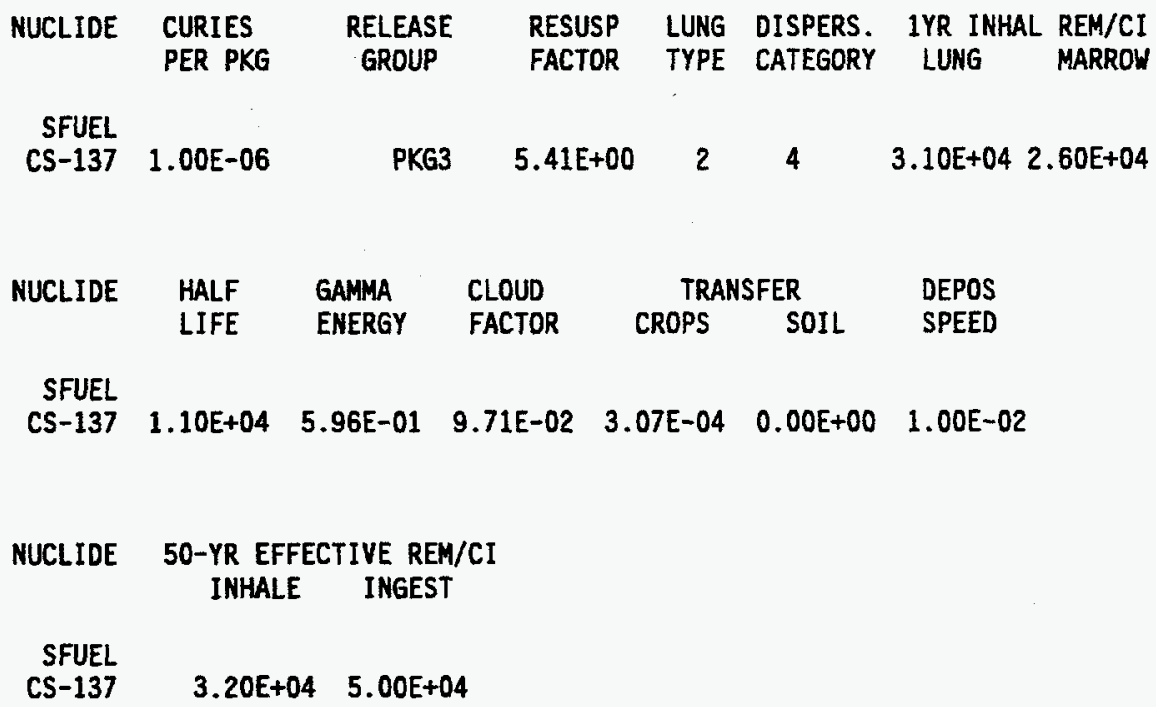

SFUEL

CS-137

$3.20 E+04 \quad 5.00 E+04$ 
_ACCIDENT_HAND_CHECK_

RELEASE RELATED DATA

ACCIDENT RATES (PER KM)

MODE RURAL SUBURBAN URBAN

TRUCK 1.000E-07 1.900E-06 $1.500 \mathrm{E}-05$

\section{RELEASE FRACTIONS}

\begin{tabular}{|c|c|c|c|c|c|c|c|}
\hline $\begin{array}{c}\text { GROUP } \\
1\end{array}$ & $\begin{array}{l}\text { SEVER: } 1 \\
0.00 E+00\end{array}$ & $\begin{array}{l}\text { SEVER: } 2 \\
1.00 E-01\end{array}$ & $\begin{array}{l}\text { SEVER: } 3 \\
1.00 E+00\end{array}$ & $\begin{array}{l}\text { SEVER: } 4 \\
1.00 E+00\end{array}$ & $\begin{array}{l}\text { SEVER: } 5 \\
3.00 E-02\end{array}$ & $\begin{array}{l}\text { SEVER: } 6 \\
1.00 E-01\end{array}$ & $\begin{array}{l}\text { SEVER: } 7 \\
1.00 \mathrm{E}+00\end{array}$ \\
\hline $\begin{array}{c}\text { GROUP } \\
1\end{array}$ & $\begin{array}{l}\text { SEVER: } 8 \\
1.00 E+00\end{array}$ & $\begin{array}{l}\text { SEVER: } 9 \\
3.00 E-02\end{array}$ & $\begin{array}{l}\text { SEVER:10 } \\
1.00 E-01\end{array}$ & $\begin{array}{l}\text { SEVER:11 } \\
1.00 E+00\end{array}$ & $\begin{array}{l}\text { SEVER:12 } \\
1.00 E+00\end{array}$ & $\begin{array}{l}\text { SEVER: } 13 \\
1.00 E+00\end{array}$ & $\begin{array}{l}\text { SEVER: } 14 \\
1.00 E+00\end{array}$ \\
\hline $\begin{array}{c}\text { GROUP } \\
1\end{array}$ & $\begin{array}{l}\text { SEVER:15 } \\
1.00 E+00\end{array}$ & $\begin{array}{l}\text { SEVER:16 } \\
1.00 E+00\end{array}$ & $\begin{array}{l}\text { SEVER:17 } \\
1.00 E+00\end{array}$ & $\begin{array}{l}\text { SEVER:18 } \\
1.00 E+00\end{array}$ & $\begin{array}{l}\text { SEVER:19 } \\
1.00 E+00\end{array}$ & $\begin{array}{l}\text { SEVER:20 } \\
1.00 E+00\end{array}$ & \\
\hline \multicolumn{8}{|c|}{ ACCIDENT SEVERITY FRACTIONS } \\
\hline $\begin{array}{c}\text { ZONE } \\
1 \\
2 \\
3\end{array}$ & $\begin{array}{l}\text { SEVER: } 1 \\
9.94 E-01 \\
0.00 E+00 \\
0.00 E+00\end{array}$ & $\begin{array}{l}\text { SEVER: } 2 \\
2.72 E-03 \\
0.00 E+00 \\
0.00 E+00\end{array}$ & $\begin{array}{l}\text { SEVER: } 3 \\
5.54 E-04 \\
0.00 E+00 \\
0.00 E+00\end{array}$ & $\begin{array}{l}\text { SEVER: } 4 \\
1.79 E-09 \\
0.00 E+00 \\
0.00 E+00\end{array}$ & $\begin{array}{l}\text { SEVER: } 5 \\
1.23 E-03 \\
0.00 E+00 \\
0.00 E+00\end{array}$ & $\begin{array}{l}\text { SEVER: } 6 \\
5.01 E-07 \\
0.00 E+00 \\
0.00 E+00\end{array}$ & $\begin{array}{l}\text { SEVER: } 7 \\
1.02 E-07 \\
0.00 E+00 \\
0.00 E+00\end{array}$ \\
\hline $\begin{array}{c}\text { ZONE } \\
1 \\
2 \\
3\end{array}$ & $\begin{array}{l}\text { SEVER: } 8 \\
3.29 E-13 \\
0.00 E+00 \\
0.00 E+00\end{array}$ & $\begin{array}{l}\text { SEVER: } 9 \\
7.95 E-04 \\
0.00 E+00 \\
0.00 E+00\end{array}$ & $\begin{array}{l}\text { SEVER:10 } \\
3.26 E-07 \\
0.00 E+00 \\
0.00 E+00\end{array}$ & $\begin{array}{l}\text { SEVER: } 11 \\
6.63 E-08 \\
0.00 E+00 \\
0.00 E+00\end{array}$ & $\begin{array}{l}\text { SEVER: } 12 \\
2.14 E-13 \\
0.00 E+00 \\
0.00 E+00\end{array}$ & $\begin{array}{l}\text { SEVER: } 13 \\
6.14 E-04 \\
0.00 E+00 \\
0.00 E+00\end{array}$ & $\begin{array}{l}\text { SEVER: } 14 \\
2.53 E-07 \\
0.00 E+00 \\
0.00 E+00\end{array}$ \\
\hline $\begin{array}{c}\text { ZONE } \\
1 \\
2 \\
3\end{array}$ & $\begin{array}{l}\text { SEVER:15 } \\
5.16 E-08 \\
0.00 E+00 \\
0.00 E+00\end{array}$ & $\begin{array}{l}\text { SEVER: } 16 \\
1.64 E-13 \\
0.00 E+00 \\
0.00 E+00\end{array}$ & $\begin{array}{l}\text { SEVER: } 17 \\
1.25 E-04 \\
0.00 E+00 \\
0.00 E+00\end{array}$ & $\begin{array}{l}\text { SEVER:18 } \\
1.08 E-08 \\
0.00 E+00 \\
0.00 E+00\end{array}$ & $\begin{array}{l}\text { SEVER:19 } \\
5.30 E-08 \\
0.00 E+00 \\
0.00 E+00\end{array}$ & $\begin{array}{l}\text { SEVER: } 20 \\
3.46 E-14 \\
1.00 E+00 \\
1.00 E+00\end{array}$ & \\
\hline
\end{tabular}


_ACCIDENT_HAND_CHECK_

\author{
AEROSOLIZED FRACTION OF RELEASED MATERIAL
}

\begin{tabular}{|c|c|c|c|c|c|c|c|}
\hline $\begin{array}{c}\text { DISP CAT } \\
1 \\
2 \\
3 \\
4 \\
5 \\
6 \\
7 \\
8 \\
9 \\
10 \\
11\end{array}$ & $\begin{array}{l}\text { SEVER: } 1 \\
0.00 E+00 \\
1.00 E-06 \\
1.00 E-02 \\
0.00 E+00 \\
1.00 E-01 \\
1.00 E+00 \\
1.00 E+00 \\
1.00 E+00 \\
1.00 E+00 \\
1.00 E+00 \\
1.00 E+00\end{array}$ & $\begin{array}{l}\text { SEVER: } 2 \\
0.00 E+00 \\
1.00 E-06 \\
1.00 E-02 \\
4.00 E-04 \\
1.00 E-01 \\
1.00 E+00 \\
1.00 E+00 \\
1.00 E+00 \\
1.00 E+00 \\
1.00 E+00 \\
1.00 E+00\end{array}$ & $\begin{array}{l}\text { SEVER: } 3 \\
0.00 E+00 \\
1.00 E-06 \\
1.00 E-02 \\
4.00 E-04 \\
1.00 E-01 \\
1.00 E+00 \\
1.00 E+00 \\
1.00 E+00 \\
1.00 E+00 \\
1.00 E+00 \\
1.00 E+00\end{array}$ & $\begin{array}{l}\text { SEVER: } 4 \\
0.00 E+00 \\
1.00 E-06 \\
1.00 E-02 \\
4.00 E-03 \\
1.00 E-01 \\
1.00 E+00 \\
1.00 E+00 \\
1.00 E+00 \\
1.00 E+00 \\
1.00 E+00 \\
1.00 E+00\end{array}$ & $\begin{array}{l}\text { SEVER: } 5 \\
0.00 E+00 \\
1.00 E-06 \\
1.00 E-02 \\
4.00 E-04 \\
1.00 E-01 \\
1.00 E+00 \\
1.00 E+00 \\
1.00 E+00 \\
1.00 E+00 \\
1.00 E+00 \\
1.00 E+00\end{array}$ & $\begin{array}{l}\text { SEVER: } 6 \\
0.00 E+00 \\
1.00 E-06 \\
1.00 E-02 \\
4.00 E-04 \\
1.00 E-01 \\
1.00 E+00 \\
1.00 E+00 \\
1.00 E+00 \\
1.00 E+00 \\
1.00 E+00 \\
1.00 E+00\end{array}$ & $\begin{array}{l}\text { SEVER: } 7 \\
0.00 E+00 \\
1.00 E-06 \\
1.00 E-02 \\
4.00 E-04 \\
1.00 E-01 \\
1.00 E+00 \\
1.00 E+00 \\
1.00 E+00 \\
1.00 E+00 \\
1.00 E+00 \\
1.00 E+00\end{array}$ \\
\hline $\begin{array}{c}\text { DISP CAT } \\
1 \\
2 \\
3 \\
4 \\
5 \\
6 \\
7 \\
8 \\
9 \\
10 \\
11\end{array}$ & $\begin{array}{l}\text { SEVER: } 8 \\
0.00 E+00 \\
1.00 E-06 \\
1.00 E-02 \\
4.00 E-03 \\
1.00 E-01 \\
1.00 E+00 \\
1.00 E+00 \\
1.00 E+00 \\
1.00 E+00 \\
1.00 E+00 \\
1.00 E+00\end{array}$ & $\begin{array}{l}\text { SEVER: } 9 \\
0.00 E+00 \\
1.00 E-06 \\
1.00 E-02 \\
4.00 E-04 \\
1.00 E-01 \\
1.00 E+00 \\
1.00 E+00 \\
1.00 E+00 \\
1.00 E+00 \\
1.00 E+00 \\
1.00 E+00\end{array}$ & $\begin{array}{l}\text { SEVER:10 } \\
0.00 E+00 \\
1.00 E-06 \\
1.00 E-02 \\
4.00 E-04 \\
1.00 E-01 \\
1.00 E+00 \\
1.00 E+00 \\
1.00 E+00 \\
1.00 E+00 \\
1.00 E+00 \\
1.00 E+00\end{array}$ & $\begin{array}{l}\text { SEVER:11 } \\
0.00 E+00 \\
1.00 E-06 \\
1.00 E-02 \\
4.00 E-04 \\
1.00 E-01 \\
1.00 E+00 \\
1.00 E+00 \\
1.00 E+00 \\
1.00 E+00 \\
1.00 E+00 \\
1.00 E+00\end{array}$ & $\begin{array}{l}\text { SEVER: } 12 \\
0.00 E+00 \\
1.00 E-06 \\
1.00 E-02 \\
4.00 E-03 \\
1.00 E-01 \\
1.00 E+00 \\
1.00 E+00 \\
1.00 E+00 \\
1.00 E+00 \\
1.00 E+00 \\
1.00 E+00\end{array}$ & $\begin{array}{l}\text { SEVER: } 13 \\
0.00 E+00 \\
1.00 E-06 \\
1.00 E-02 \\
4.00 E-04 \\
1.00 E-01 \\
1.00 E+00 \\
1.00 E+00 \\
1.00 E+00 \\
1.00 E+00 \\
1.00 E+00 \\
1.00 E+00\end{array}$ & $\begin{array}{l}\text { SEVER : } 14 \\
0.00 E+00 \\
1.00 E-06 \\
1.00 E-02 \\
4.00 E-04 \\
1.00 E-01 \\
1.00 E+00 \\
1.00 E+00 \\
1.00 E+00 \\
1.00 E+00 \\
1.00 E+00 \\
1.00 E+00\end{array}$ \\
\hline $\begin{array}{c}\text { DISP CAT } \\
1 \\
2 \\
3 \\
4 \\
5 \\
6 \\
7 \\
8 \\
9 \\
10 \\
11\end{array}$ & $\begin{array}{l}\text { SEVER:15 } \\
0.00 E+00 \\
1.00 E-06 \\
1.00 E-02 \\
4.00 E-04 \\
1.00 E-01 \\
1.00 E+00 \\
1.00 E+00 \\
1.00 E+00 \\
1.00 E+00 \\
1.00 E+00 \\
1.00 E+00\end{array}$ & $\begin{array}{l}\text { SEVER: } 16 \\
0.00 E+00 \\
1.00 E-06 \\
1.00 E-02 \\
4.00 E-03 \\
1.00 E-01 \\
1.00 E+00 \\
1.00 E+00 \\
1.00 E+00 \\
1.00 E+00 \\
1.00 E+00 \\
1.00 E+00\end{array}$ & $\begin{array}{l}\text { SEVER: } 17 \\
0.00 E+00 \\
1.00 E-06 \\
1.00 E-02 \\
4.00 E-03 \\
1.00 E-01 \\
1.00 E+00 \\
1.00 E+00 \\
1.00 E+00 \\
1.00 E+00 \\
1.00 E+00 \\
1.00 E+00\end{array}$ & $\begin{array}{l}\text { SEVER:18 } \\
0.00 E+00 \\
1.00 E-06 \\
1.00 E-02 \\
4.00 E-03 \\
1.00 E-01 \\
1.00 E+00 \\
1.00 E+00 \\
1.00 E+00 \\
1.00 E+00 \\
1.00 E+00 \\
1.00 E+00\end{array}$ & $\begin{array}{l}\text { SEVER:19 } \\
0.00 E+00 \\
1.00 E-06 \\
1.00 E-02 \\
4.00 E-03 \\
1.00 E-01 \\
1.00 E+00 \\
1.00 E+00 \\
1.00 E+00 \\
1.00 E+00 \\
1.00 E+00 \\
1.00 E+00\end{array}$ & $\begin{array}{l}\text { SEVER:20 } \\
0.00 E+00 \\
1.00 E-06 \\
1.00 E-02 \\
4.00 E-03 \\
1.00 E-01 \\
1.00 E+00 \\
1.00 E+00 \\
1.00 E+00 \\
1.00 E+00 \\
1.00 E+00 \\
1.00 E+00\end{array}$ & \\
\hline
\end{tabular}


_ACCIDENT_HAND_CHECK_

FRACTION OF AEROSOLS BELOW 10 MICRONS AED

\begin{tabular}{|c|c|c|c|c|c|c|c|}
\hline $\begin{array}{c}\text { DISP CAT } \\
1 \\
2 \\
3 \\
4 \\
5 \\
6 \\
7 \\
8 \\
9 \\
10 \\
11\end{array}$ & $\begin{array}{l}\text { SEVER: } 1 \\
0.00 E+00 \\
5.00 E-02 \\
5.00 E-02 \\
0.00 E+00 \\
5.00 E-02 \\
5.00 E-02 \\
1.00 E+00 \\
1.00 E+00 \\
1.00 E+00 \\
1.00 E+00 \\
1.00 E+00\end{array}$ & $\begin{array}{l}\text { SEVER: } 2 \\
0.00 E+00 \\
5.00 E-02 \\
5.00 E-02 \\
5.00 E-01 \\
5.00 E-02 \\
5.00 E-02 \\
1.00 E+00 \\
1.00 E+00 \\
1.00 E+00 \\
1.00 E+00 \\
1.00 E+00\end{array}$ & $\begin{array}{l}\text { SEVER: } 3 \\
0.00 E+00 \\
5.00 E-02 \\
5.00 E-02 \\
5.00 E-01 \\
5.00 E-02 \\
5.00 E-02 \\
1.00 E+00 \\
1.00 E+00 \\
1.00 E+00 \\
1.00 E+00 \\
1.00 E+00\end{array}$ & $\begin{array}{l}\text { SEVER: } 4 \\
0.00 E+00 \\
5.00 E-02 \\
5.00 E-02 \\
5.00 E-01 \\
5.00 E-02 \\
5.00 E-02 \\
1.00 E+00 \\
1.00 E+00 \\
1.00 E+00 \\
1.00 E+00 \\
1.00 E+00\end{array}$ & $\begin{array}{l}\text { SEVER: } 5 \\
0.00 E+00 \\
5.00 E-02 \\
5.00 E-02 \\
5.00 E-01 \\
5.00 E-02 \\
5.00 E-02 \\
1.00 E+00 \\
1.00 E+00 \\
1.00 E+00 \\
1.00 E+00 \\
1.00 E+00\end{array}$ & $\begin{array}{l}\text { SEVER: } 6 \\
0.00 \mathrm{E}+00 \\
5.00 \mathrm{E}-02 \\
5.00 \mathrm{E}-02 \\
5.00 \mathrm{E}-01 \\
5.00 \mathrm{E}-02 \\
5.00 \mathrm{E}-02 \\
1.00 \mathrm{E}+00 \\
1.00 \mathrm{E}+00 \\
1.00 \mathrm{E}+00 \\
1.00 \mathrm{E}+00 \\
1.00 \mathrm{E}+00\end{array}$ & $\begin{array}{l}\text { SEVER: } 7 \\
0.00 E+00 \\
5.00 E-02 \\
5.00 E-02 \\
5.00 E-01 \\
5.00 E-02 \\
5.00 E-02 \\
1.00 E+00 \\
1.00 E+00 \\
1.00 E+00 \\
1.00 E+00 \\
1.00 E+00\end{array}$ \\
\hline $\begin{array}{c}\text { DISP CAT } \\
1 \\
2 \\
3 \\
4 \\
5 \\
6 \\
7 \\
8 \\
9 \\
10 \\
11\end{array}$ & $\begin{array}{l}\text { SEVER: } 8 \\
0.00 E+00 \\
5.00 E-02 \\
5.00 E-02 \\
5.00 E-01 \\
5.00 E-02 \\
5.00 E-02 \\
1.00 E+00 \\
1.00 E+00 \\
1.00 E+00 \\
1.00 E+00 \\
1.00 E+00\end{array}$ & $\begin{array}{l}\text { SEVER: } 9 \\
0.00 E+00 \\
5.00 E-02 \\
5.00 E-02 \\
5.00 E-01 \\
5.00 E-02 \\
5.00 E-02 \\
1.00 E+00 \\
1.00 E+00 \\
1.00 E+00 \\
1.00 E+00 \\
1.00 E+00\end{array}$ & $\begin{array}{l}\text { SEVER:10 } \\
0.00 E+00 \\
5.00 E-02 \\
5.00 E-02 \\
5.00 E-01 \\
5.00 E-02 \\
5.00 E-02 \\
1.00 E+00 \\
1.00 E+00 \\
1.00 E+00 \\
1.00 E+00 \\
1.00 E+00\end{array}$ & $\begin{array}{l}\text { SEVER: } 11 \\
0.00 E+00 \\
5.00 E-02 \\
5.00 E-02 \\
5.00 E-01 \\
5.00 E-02 \\
5.00 E-02 \\
1.00 E+00 \\
1.00 E+00 \\
1.00 E+00 \\
1.00 E+00 \\
1.00 E+00\end{array}$ & $\begin{array}{l}\text { SEVER:12 } \\
0.00 E+00 \\
5.00 E-02 \\
5.00 E-02 \\
5.00 E-01 \\
5.00 E-02 \\
5.00 E-02 \\
1.00 E+00 \\
1.00 E+00 \\
1.00 E+00 \\
1.00 E+00 \\
1.00 E+00\end{array}$ & $\begin{array}{l}\text { SEVER: } 13 \\
0.00 E+00 \\
5.00 E-02 \\
5.00 E-02 \\
5.00 E-01 \\
5.00 E-02 \\
5.00 E-02 \\
1.00 E+00 \\
1.00 E+00 \\
1.00 E+00 \\
1.00 E+00 \\
1.00 E+00\end{array}$ & $\begin{array}{l}\text { SEVER:14 } \\
0.00 E+00 \\
5.00 E-02 \\
5.00 E-02 \\
5.00 E-01 \\
5.00 E-02 \\
5.00 E-02 \\
1.00 E+00 \\
1.00 E+00 \\
1.00 E+00 \\
1.00 E+00 \\
1.00 E+00\end{array}$ \\
\hline $\begin{array}{c}\text { DISP CAT } \\
1 \\
2 \\
3 \\
4 \\
5 \\
6 \\
7 \\
8 \\
9 \\
10 \\
11\end{array}$ & $\begin{array}{l}\text { SEVER:15 } \\
0.00 E+00 \\
5.00 E-02 \\
5.00 E-02 \\
5.00 E-01 \\
5.00 E-02 \\
5.00 E-02 \\
1.00 E+00 \\
1.00 E+00 \\
1.00 E+00 \\
1.00 E+00 \\
1.00 E+00\end{array}$ & $\begin{array}{l}\text { SEVER:16 } \\
0.00 E+00 \\
5.00 E-02 \\
5.00 E-02 \\
5.00 E-01 \\
5.00 E-02 \\
5.00 E-02 \\
1.00 E+00 \\
1.00 E+00 \\
1.00 E+00 \\
1.00 E+00 \\
1.00 E+00\end{array}$ & $\begin{array}{l}\text { SEVER:17 } \\
0.00 E+00 \\
5.00 E-02 \\
5.00 E-02 \\
5.00 E-01 \\
5.00 E-02 \\
5.00 E-02 \\
1.00 E+00 \\
1.00 E+00 \\
1.00 E+00 \\
1.00 E+00 \\
1.00 E+00\end{array}$ & $\begin{array}{l}\text { SEVER:18 } \\
0.00 E+00 \\
5.00 E-02 \\
5.00 E-02 \\
5.00 E-01 \\
5.00 E-02 \\
5.00 E-02 \\
1.00 E+00 \\
1.00 E+00 \\
1.00 E+00 \\
1.00 E+00 \\
1.00 E+00\end{array}$ & $\begin{array}{l}\text { SEVER:19 } \\
0.00 E+00 \\
5.00 E-02 \\
5.00 E-02 \\
5.00 E-01 \\
5.00 E-02 \\
5.00 E-02 \\
1.00 E+00 \\
1.00 E+00 \\
1.00 E+00 \\
1.00 E+00 \\
1.00 E+00\end{array}$ & $\begin{array}{l}\text { SEVER: } 20 \\
0.00 E+00 \\
5.00 E-02 \\
5.00 E-02 \\
5.00 E-01 \\
5.00 E-02 \\
5.00 E-02 \\
1.00 E+00 \\
1.00 E+00 \\
1.00 E+00 \\
1.00 E+00 \\
1.00 E+00\end{array}$ & \\
\hline
\end{tabular}


ACCIDENT_HAND_CHECK_

COST RELATED DATA

EMERGENCY RESPONSE COST

$\begin{array}{lllllll}\text { SEVER: } 1 & \text { SEVER: } 2 & \text { SEVER: } 3 & \text { SEVER: } 4 & \text { SEVER: } 5 & \text { SEVER: } 6 & \text { SEVER: } 7 \\ 0.00 E+00 & 0.00 E+00 & 0.00 E+00 & 0.00 E+00 & 0.00 E+00 & 0.00 E+00 & 0.00 E+00 \\ & & & & & & \\ \text { SEVER: } 8 & \text { SEVER: } 9 & \text { SEVER:10 } & \text { SEVER:11 } & \text { SEVER:12 } & \text { SEVER:13 } & \text { SEVER:14 } \\ 0.00 E+00 & 0.00 E+00 & 0.00 E+00 & 0.00 E+00 & 0.00 E+00 & 0.00 E+00 & 0.00 E+00 \\ & & & & & & \\ \text { SEVER:15 } & \text { SEVER:16 } & \text { SEVER:17 } & \text { SEVER:18 } & \text { SEVER:19 } & \text { SEVER:20 } \\ 0.00 E+00 & 0.00 E+00 & 0.00 E+00 & 0.00 E+00 & 0.00 E+00 & 0.00 E+00 & \end{array}$

ON-SCENE COSTS

(RF=RELEASE FRACTION)

$R F=0$.

0.

$0 .<R F<=.01$

0 .

$.01<R F<=0.1$ $.1<\mathrm{RF}<=1$.

. 0 . 
RUN DATE: [ 25-MAR-95 AT 14:06:57 ]

_ACCIDENT_HAND_CHECK_

HEALTH RELATED DATA

\section{EARLY FATALITY PROBABILITIES}

$\begin{array}{rrrrr}\text { DOSE (REM) } & \text { LUNG-1 } & \text { LUNG-2 } & \text { LUNG }-3 & \text { MARROW } \\ 100000.000 & 1.000 E+00 & 1.000 E+00 & 1.000 E+00 & 1.000 E+00 \\ 80000.000 & 1.000 E+00 & 8.500 E-01 & 8.000 E-01 & 1.000 E+00 \\ 70000.000 & 1.000 E+00 & 8.000 E-01 & 5.000 E-01 & 1.000 E+00 \\ 40000.000 & 1.000 E+00 & 7.000 E-01 & 0.000 E+00 & 1.000 E+00 \\ 30000.000 & 1.000 E+00 & 5.000 E-01 & 0.000 E+00 & 1.000 E+00 \\ 25000.000 & 1.000 E+00 & 2.000 E-01 & 0.000 E+00 & 1.000 E+00 \\ 20000.000 & 1.000 E+00 & 8.000 E-02 & 0.000 E+00 & 1.000 E+00 \\ 10000.000 & 6.000 E-01 & 0.000 E+00 & 0.000 E+00 & 1.000 E+00 \\ 8000.000 & 1.000 E-01 & 0.000 E+00 & 0.000 E+00 & 1.000 E+00 \\ 6000.000 & 6.000 E-02 & 0.000 E+00 & 0.000 E+00 & 1.000 E+00 \\ 4000.000 & 3.000 E-02 & 0.000 E+00 & 0.000 E+00 & 1.000 E+00 \\ 3000.000 & 0.000 E+00 & 0.000 E+00 & 0.000 E+00 & 1.000 E+00 \\ 2000.000 & 0.000 E+00 & 0.000 E+00 & 0.000 E+00 & 1.000 E+00 \\ 1000.000 & 0.000 E+00 & 0.000 E+00 & 0.000 E+00 & 1.000 E+00 \\ 800.000 & 0.000 E+00 & 0.000 E+00 & 0.000 E+00 & 9.960 E-01 \\ 700.000 & 0.000 E+00 & 0.000 E+00 & 0.000 E+00 & 9.000 E-01 \\ 600.000 & 0.000 E+00 & 0.000 E+00 & 0.000 E+00 & 4.000 E-01 \\ 500.000 & 0.000 E+00 & 0.000 E+00 & 0.000 E+00 & 5.000 E-02 \\ 400.000 & 0.000 E+00 & 0.000 E+00 & 0.000 E+00 & 0.000 E+00 \\ 300.000 & 0.000 E+00 & 0.000 E+00 & 0.000 E+00 & 0.000 E+00 \\ 100.000 & 0.000 E+00 & 0.000 E+00 & 0.000 E+00 & 0.000 E+00 \\ 75.000 & 0.000 E+00 & 0.000 E+00 & 0.000 E+00 & 0.000 E+00 \\ 50.000 & 0.000 E+00 & 0.000 E+00 & 0.000 E+00 & 0.000 E+00 \\ 30.000 & 0.000 E+00 & 0.000 E+00 & 0.000 E+00 & 0.000 E+00 \\ 15.000 & 0.000 E+00 & 0.000 E+00 & 0.000 E+00 & 0.000 E+00 \\ 5.000 & 0.000 E+00 & 0.000 E+00 & 0.000 E+00 & 0.000 E+00 \\ 1.000 & 0.000 E+00 & 0.000 E+00 & 0.000 E+00 & 0.000 E+00 \\ 0.100 & 0.000 E+00 & 0.000 E+00 & 0.000 E+00 & 0.000 E+00 \\ 0.010 & 0.000 E+00 & 0.000 E+00 & 0.000 E+00 & 0.000 E+00 \\ 0.010 & 0.000 E+00 & 0.000 E+00 & 0.000 E+00 & 0.000 E+00\end{array}$


RUN DATE: [ 25-MAR-95 AT 14:06:57]

ACCIDENT_HAND_CHECK_

DISPERSAL ACCIDENT INPUT

$\begin{array}{cc}\text { AREADA } & \text { DILUTION } \\ \text { (M SQ) } & \text { FACTOR* } \\ 4.590 E+02 & 3.420 E-03 \\ 1.530 E+03 & 1.720 E-03\end{array}$

* DILUTION FACTOR UNITS ARE (CI-SEC/M**3/CI-RELEASED)

NON-DISPERSAL ACCIDENT INPUT

RADIST(M)

RURAL SUBURBAN URBAN

$3.050 E+00 \quad 3.050 E+00 \quad 3.050 E+00$

$6.100 E+00 \quad 6.100 E+00 \quad 6.100 E+00$

$9.100 E+00 \quad 9.100 E+00 \quad 9.100 E+00$

$1.220 E+01 \quad 1.220 E+01 \quad 1.220 E+01$

$1.520 \mathrm{E}+01 \quad 1.520 \mathrm{E}+01 \quad 1.520 \mathrm{E}+01$

$3.050 E+01 \quad 3.050 E+01 \quad 3.050 E+01$

$6.100 E+01 \quad 6.100 E+01 \quad 6.100 E+01$

$9.140 E+01 \quad 9.140 E+01 \quad 9.140 E+01$

$1.524 \mathrm{E}+02 \quad 1.524 \mathrm{E}+02 \quad 1.524 \mathrm{E}+02$

$3.050 E+02 \quad 3.050 E+02 \quad 3.050 E+02$

BUILDING DOSE FACTOR

FRACTION OF LAND UNOER CULTIVATION

CONTAMINATION CLEAN UP LEVEL (UCI/M**2)

BREATHING RATE ( $\left.M^{\star *} 3 / \mathrm{SEC}\right)$
$=8.600 E-03$

$=1.000 E+00$

$=2.000 \mathrm{E}-01$

$=3.300 \mathrm{E}-04$ 
RUN DATE: [ 25-MAR-95 AT 14:06:57]

ACCIDENT_HAND_CHECK_

\section{REGULATORY CHECKS}

FOR THE SHIPMENT OF SFUEL BY MODE 1

THE DOSE RATE AT 2 METERS COULD EXCEED 10 MR/HR

PPS*TI HAS BEEN RESET TO EQUAL 13.00 
RUN DATE: [ 25-MAR-95 AT 14:06:57]

_ACCIDENT_HAND_CHECK_

CALCULATIONAL INFORMATION FOR MODE

TRUCK 
RUN DATE: [ 25-MAR-95 AT 14:06:57]

ACCIDENT_HAND_CHECK_

MODE TRUCK

1-YEAR LUNG DOSE - INHALATION PATHWAY

$B D F=1 \quad$ (REM)

$\begin{array}{cllllllll}\text { AREA \# } & \text { SEVER: } 1 & \text { SEVER: } 2 & \text { SEVER: } 3 & \text { SEVER: } 4 & \text { SEVER: } 5 & \text { SEVER: } 6 & \text { SEVER: } 7 \\ 1 & 0.00 E+00 & 7.00 E-13 & 7.00 E-12 & 7.00 E-11 & 2.10 E-13 & 7.00 E-13 & 7.00 E-12 \\ 2 & 0.00 E+00 & 3.51 E-13 & 3.51 E-12 & 3.51 E-11 & 1.05 E-13 & 3.51 E-13 & 3.51 E-12 \\ & & & & & & & \\ \text { AREA \# } & \text { SEVER: } 8 & \text { SEVER: } 9 & \text { SEVER:10 } & \text { SEVER:11 } & \text { SEVER:12 } & \text { SEVER: } 13 & \text { SEVER:14 } \\ 1 & 7.00 E-11 & 2.10 E-13 & 7.00 E-13 & 7.00 E-12 & 7.00 E-11 & 7.00 E-12 & 7.00 E-12 \\ 2 & 3.51 E-11 & 1.05 E-13 & 3.51 E-13 & 3.51 E-12 & 3.51 E-11 & 3.51 E-12 & 3.51 E-12 \\ & & & & & & & \\ \text { AREA \# } & \text { SEVER:15 } & \text { SEVER:16 } & \text { SEVER:17 } & \text { SEVER:18 } & \text { SEVER:19 } & \text { SEVER:20 } & \\ 1 & 7.00 E-12 & 7.00 E-11 & 7.00 E-11 & 7.00 E-11 & 7.00 E-11 & 7.00 E-11 & \\ 2 & 3.51 E-12 & 3.51 E-11 & 3.51 E-11 & 3.51 E-11 & 3.51 E-11 & 3.51 E-11 & \end{array}$


_ACCIDENT_HAND_CHECK_

1-YEAR MARROW DOSE - INHALATION PATHMAY

$$
B D F=1 \quad \text { (REM) }
$$

$\begin{array}{clllllll}\text { AREA \# } & \text { SEVER: } 1 & \text { SEVER: } 2 & \text { SEVER: } 3 & \text { SEVER: } 4 & \text { SEVER: } 5 & \text { SEVER: } 6 & \text { SEVER: } 7 \\ 1 & 0.00 E+00 & 5.87 E-13 & 5.87 E-12 & 5.87 E-11 & 1.76 E-13 & 5.87 E-13 & 5.87 E-12 \\ 2 & 0.00 E+00 & 2.95 E-13 & 2.95 E-12 & 2.95 E-11 & 8.84 E-14 & 2.95 E-13 & 2.95 E-12 \\ & & & & & & & \\ \text { AREA * } & \text { SEVER: } 8 & \text { SEVER: } 9 & \text { SEVER:10 } & \text { SEVER:11 } & \text { SEVER:12 } & \text { SEVER:13 } & \text { SEVER:14 } \\ 1 & 5.87 E-11 & 1.76 E-13 & 5.87 E-13 & 5.87 E-12 & 5.87 E-11 & 5.87 E-12 & 5.87 E-12 \\ 2 & 2.95 E-11 & 8.84 E-14 & 2.95 E-13 & 2.95 E-12 & 2.95 E-11 & 2.95 E-12 & 2.95 E-12 \\ & & & & & & & \\ \text { AREA \# } & \text { SEVER:15 } & \text { SEVER:16 } & \text { SEVER:17 } & \text { SEVER:18 } & \text { SEVER:19 } & \text { SEVER:20 } & \\ 1 & 5.87 E-12 & 5.87 E-11 & 5.87 E-11 & 5.87 E-11 & 5.87 E-11 & 5.87 E-11 & \\ 2 & 2.95 E-12 & 2.95 E-11 & 2.95 E-11 & 2.95 E-11 & 2.95 E-11 & 2.95 E-11 & \end{array}$


RUN DATE: [ 25-MAR-95 AT 14:06:57 ]

_ACCIDENT_HAND_CHECK

MODE TRUCK

GROUND SURFACE CONTAMINATION TABLE (MICRO CI/M**2) BEFORE CLEANUP

$\begin{array}{cllllllll}\text { AREA \# } & \text { SEVER: } 1 & \text { SEVER: } 2 & \text { SEVER: } 3 & \text { SEVER: } 4 & \text { SEVER: } 5 & \text { SEVER: } 6 & \text { SEVER: } 7 \\ 1 & 0.00 E+00 & 1.37 E-09 & 1.37 E-08 & 1.37 E-07 & 4.10 E-10 & 1.37 E-09 & 1.37 E-08 \\ 2 & 0.00 E+00 & 6.87 E-10 & 6.87 E-09 & 6.87 E-08 & 2.06 E-10 & 6.87 E-10 & 6.87 E-09 \\ & & & & & & & \\ \text { AREA \# } & \text { SEVER: } 8 & \text { SEVER: } 9 & \text { SEVER:10 } & \text { SEVER:11 } & \text { SEVER:12 } & \text { SEVER:13 } & \text { SEVER:14 } \\ 1 & 1.37 E-07 & 4.10 E-10 & 1.37 E-09 & 1.37 E-08 & 1.37 E-07 & 1.37 E-08 & 1.37 E-08 \\ 2 & 6.87 E-08 & 2.06 E-10 & 6.87 E-10 & 6.87 E-09 & 6.87 E-08 & 6.87 E-09 & 6.87 E-09 \\ & & & & & & & \\ \text { AREA \# } & \text { SEVER:15 } & \text { SEVER:16 } & \text { SEVER:17 } & \text { SEVER:18 } & \text { SEVER:19 } & \text { SEVER:20 } & \\ 1 & 1.37 E-08 & 1.37 E-07 & 1.37 E-07 & 1.37 E-07 & 1.37 E-07 & 1.37 E-07 & \\ 2 & 6.87 E-09 & 6.87 E-08 & 6.87 E-08 & 6.87 E-08 & 6.87 E-08 & 6.87 E-08 & \end{array}$


_ACCIDENT_HAND_CHECK_

\section{INCIDENT-FREE SUMMARY

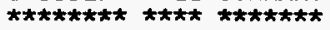

INCIDENT-FREE POPULATION EXPOSURE IN PERSON-REM

PASSENGR CREN HANDLERS OFF LINK ON LINK STOPS STORAGE TOTALS LINK $10.00 \mathrm{E}+00$ 3.55E-05 $0.00 \mathrm{E}+00 \quad 2.06 \mathrm{E}-07 \quad 1.19 \mathrm{E}-05$ 9.60E-01 $0.00 \mathrm{E}+00 \quad 9.60 \mathrm{E}-01$

TOTALS: $0.00 E+00 \quad 3.55 E-05 \quad 0.00 E+00 \quad 2.06 E-07 \quad 1.19 E-05 \quad 9.60 E-01 \quad 0.00 E+00 \quad 9.60 E-01$

MAXIMUM INDIVIDUAL IN-TRANSIT DOSE

LINK 1 5.96E-07 REM 
_ACCIDENT_HAND_CHECK_

INCIDENT-FREE IMPORTANCE ANALYSIS SUMMARY FOR LINK 1

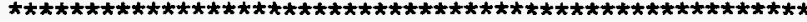

DESCRIPTION OF PARAMETER

1 DOSE RATE (TRANSPORT INDEX)

2 K ZERO

3 NUMBER OF SHIPMENTS

4 PACKAGES PER SHIPMENT

5 DISTANCE TRAVELED

6 PERSONS EXPOSED WHILE STOPPED

7 STOP TIME

FRACTION OF TRAVEL - RURAL

NUMBER OF CREN MEMBERS

NUMBER OF PEOPLE PER VEHICLE

TRAFFIC COUNT - RURAL

POPULATION DENSITY - RURAL

FRACTION OF RUSH HOUR TRAVEL

STORAGE EXPOSURE DISTANCE

NUMBER OF HANDLINGS

EXPOSURE TIME FOR HANDLERS

PERSONS EXPOSED PER HANDLING

NUMBER OF FLIGHT ATTENDANTS

TRAFFIC COUNT - URBAN

TRAFFIC COUNT - SUBURBAN

HANDLER EXPOSURE DISTANCE

NUMBER OF PERSONS EXPOSED DURING STORAGE

STORAGE TIME PER SHIPMENT

SUBURBAN SHIELDING FACTOR (RS)

VELOCITY - SUBURBAN

POPULATION DENSITY - SUBURBAN

FRACTION OF TRAVEL - SUBURBAN

RATIO OF PEDESTRIAN DENSITY (RPD)

FRACTION OF TRAVEL - URBAN

POPULATION DENSITY - URBAN

URBAN SHIELDING FACTOR (RU)

FRACTION OF TRAVEL ON CITY STREETS

RURAL SHIELDING FACTOR (RR)

VELOCITY - URBAN

FRACTION OF TRAVEL ON FREEWAYS

VELOCITY - RURAL

DISTANCE FROM SOURCE TO CREW

EXPOSURE DISTANCE WHILE STOPPED
IMPORTANCE

9.596E-03

9.596E-03

9.596E-03

$9.596 \mathrm{E}-03$

$9.596 \mathrm{E}-03$

9.596E-03

9.596E-03

4.767E-07

3.552E-07

$1.194 \mathrm{E}-07$

1.194E-07

2.057E-09

9. $237 \mathrm{E}-16$

$0.000 \mathrm{E}+00$

$0.000 E+00$

$0.000 E+00$

$0.000 E+00$

$0.000 E+00$

$0.000 E+00$

$0.000 E+00$

$0.000 E+00$

$0.000 E+00$

$0.000 E+00$

$0.000 E+00$

$0.000 E+00$

$0.000 E+00$

$0.000 E+00$

$0.000 E+00$

$0.000 E+00$

$0.000 E+00$

$0.000 E+00$

$0.000 E+00$

$0.000 E+00$

$0.000 E+00$

$-1.089 \mathrm{E}-07$

$-5.961 E-07$

$-6.594 E-07$

$-1.919 E-02$

THE IMPORTANCE VALUE ESTIMATES THE PERSON-REM INFLUENCE OF A ONE PERCENT INCREASE IN THE PARAMETER 
_ACCIDENT_HAND_CHECK_

ACCIDENT SUMMARY

NUMBER OF EXPECTED ACCIDENTS -- MODE TRUCK

$\begin{array}{clll}\text { CATEGORY } & \text { RURAL } & \text { SUBURB } & \text { URBAN } \\ 1 & 9.94 E-08 & 0.00 E+00 & 0.00 E+00 \\ 2 & 2.72 E-10 & 0.00 E+00 & 0.00 E+00 \\ 3 & 5.54 E-11 & 0.00 E+00 & 0.00 E+00 \\ 4 & 1.79 E-16 & 0.00 E+00 & 0.00 E+00 \\ 5 & 1.23 E-10 & 0.00 E+00 & 0.00 E+00 \\ 6 & 5.01 E-14 & 0.00 E+00 & 0.00 E+00 \\ 7 & 1.02 E-14 & 0.00 E+00 & 0.00 E+00 \\ 8 & 3.29 E-20 & 0.00 E+00 & 0.00 E+00 \\ 9 & 7.95 E-11 & 0.00 E+00 & 0.00 E+00 \\ 10 & 3.26 E-14 & 0.00 E+00 & 0.00 E+00 \\ 11 & 6.63 E-15 & 0.00 E+00 & 0.00 E+00 \\ 12 & 2.14 E-20 & 0.00 E+00 & 0.00 E+00 \\ 13 & 6.14 E-11 & 0.00 E+00 & 0.00 E+00 \\ 14 & 2.53 E-14 & 0.00 E+00 & 0.00 E+00 \\ 15 & 5.16 E-15 & 0.00 E+00 & 0.00 E+00 \\ 16 & 1.64 E-20 & 0.00 E+00 & 0.00 E+00 \\ 17 & 1.25 E-11 & 0.00 E+00 & 0.00 E+00 \\ 18 & 1.08 E-15 & 0.00 E+00 & 0.00 E+00 \\ 19 & 5.30 E-15 & 0.00 E+00 & 0.00 E+00 \\ 20 & 3.46 E-21 & 0.00 E+00 & 0.00 E+00\end{array}$


RUM DATE: [ 25-MAR-95 AT 14:06:57]

ACCIDENT_HAND_CHECK

\section{EARLY FATALITY CONSEQUENCES -- MODE}

CATEG
1
2
3
4
5
6
7
8
9
10
11
12
13
14
15
16
17
18
19
20

RURAL

$0.00 E+00$

$0.00 E+00$

$0.00 \mathrm{E}+00$

$0.00 E+00$

$0.00 E+00$

$0.00 E+00$

$0.00 \mathrm{E}+00$

$0.00 E+00$

$0.00 E+00$

$0.00 E+00$

$0.00 E+00$

$0.00 E+00$

$0.00 \mathrm{E}+00$

$0.00 E+00$

$0.00 E+00$

$0.00 E+00$

$0.00 E+00$

$0.00 E+00$

$0.00 \mathrm{E}+00$

$0.00 \mathrm{E}+00$
SUBURB

$0.00 E+00$

$0.00 E+00$

$0.00 E+00$

$0.00 E+00$

$0.00 E+00$

$0.00 E+00$

$0.00 \mathrm{E}+00$

$0.00 E+00$

$0.00 E+00$

$0.00 E+00$

$0.00 E+00$

$0.00 E+00$

$0.00 E+00$

$0.00 E+00$

D. $00 E+00$

$0.00 E+00$

$0.00 \mathrm{E}+00$

$0.00 E+00$

$0.00 \mathrm{E}+00$

$0.00 E+00$
TRUCK

URBAN

$0.00 E+00$

$0.00 E+00$

$0.00 E+00$

$0.00 E+00$

$0.00 E+00$

$0.00 E+00$

$0.00 E+00$

$0.00 E+00$

$0.00 E+00$

$0.00 E+00$

$0.00 E+00$

$0.00 E+00$

$0.00 E+00$

$0.00 E+00$

$0.00 E+00$

$0.00 E+00$

$0.00 E+00$

$0.00 E+00$

$0.00 E+00$

$0.00 E+00$ 
_ACCIDENT_HAND_CHECK_

ECONOMIC CONSEQUENCES -- MODE TRUCK

$\begin{array}{clll}\text { CATEGORY } & \text { RURAL } & \text { SUBURB } & \text { URBAN } \\ 1 & 0.00 E+00 & 0.00 E+00 & 0.00 E+00 \\ 2 & 0.00 E+00 & 0.00 E+00 & 0.00 E+00 \\ 3 & 0.00 E+00 & 0.00 E+00 & 0.00 E+00 \\ 4 & 0.00 E+00 & 0.00 E+00 & 0.00 E+00 \\ 5 & 0.00 E+00 & 0.00 E+00 & 0.00 E+00 \\ 6 & 0.00 E+00 & 0.00 E+00 & 0.00 E+00 \\ 7 & 0.00 E+00 & 0.00 E+00 & 0.00 E+00 \\ 8 & 0.00 E+00 & 0.00 E+00 & 0.00 E+00 \\ 9 & 0.00 E+00 & 0.00 E+00 & 0.00 E+00 \\ 10 & 0.00 E+00 & 0.00 E+00 & 0.00 E+00 \\ 11 & 0.00 E+00 & 0.00 E+00 & 0.00 E+00 \\ 12 & 0.00 E+00 & 0.00 E+00 & 0.00 E+00 \\ 13 & 0.00 E+00 & 0.00 E+00 & 0.00 E+00 \\ 14 & 0.00 E+00 & 0.00 E+00 & 0.00 E+00 \\ 15 & 0.00 E+00 & 0.00 E+00 & 0.00 E+00 \\ 16 & 0.00 E+00 & 0.00 E+00 & 0.00 E+00 \\ 17 & 0.00 E+00 & 0.00 E+00 & 0.00 E+00 \\ 18 & 0.00 E+00 & 0.00 E+00 & 0.00 E+00 \\ 19 & 0.00 E+00 & 0.00 E+00 & 0.00 E+00 \\ 20 & 0.00 E+00 & 0.00 E+00 & 0.00 E+00\end{array}$


_ACCIDENT_HAND_CHECK

RADIOLOGICAL CONSEQUENCES -- MODE TRUCK

50 YEAR POPULATION DOSE IN PERSON REM

$\begin{array}{clll}\text { CATEGORY } & \text { RURAL } & \text { SUBURB } & \text { URBAN } \\ 1 & 0.00 E+00 & 0.00 E+00 & 0.00 E+00 \\ 2 & 2.64 E-11 & 6.52 E-10 & 2.12 E-09 \\ 3 & 2.64 E-10 & 6.52 E-09 & 2.12 E-08 \\ 4 & 2.64 E-09 & 6.52 E-08 & 2.12 E-07 \\ 5 & 7.91 E-12 & 1.96 E-10 & 6.35 E-10 \\ 6 & 2.64 E-11 & 6.52 E-10 & 2.12 E-09 \\ 7 & 2.64 E-10 & 6.52 E-09 & 2.12 E-08 \\ 8 & 2.64 E-09 & 6.52 E-08 & 2.12 E-07 \\ 9 & 7.91 E-12 & 1.96 E-10 & 6.35 E-10 \\ 10 & 2.64 E-11 & 6.52 E-10 & 2.12 E-09 \\ 11 & 2.64 E-10 & 6.52 E-09 & 2.12 E-08 \\ 12 & 2.64 E-09 & 6.52 E-08 & 2.12 E-07 \\ 13 & 2.64 E-10 & 6.52 E-09 & 2.12 E-08 \\ 14 & 2.64 E-10 & 6.52 E-09 & 2.12 E-08 \\ 15 & 2.64 E-10 & 6.52 E-09 & 2.12 E-08 \\ 16 & 2.64 E-09 & 6.52 E-08 & 2.12 E-07 \\ 17 & 2.64 E-09 & 6.52 E-08 & 2.12 E-07 \\ 18 & 2.64 E-09 & 6.52 E-08 & 2.12 E-07 \\ 19 & 2.64 E-09 & 6.52 E-08 & 2.12 E-07 \\ 20 & 2.64 E-09 & 6.52 E-08 & 2.12 E-07\end{array}$


RUN DATE: [ 25-MAR-95 AT 14:06:57]

_ACCIDENT_HAND_CHECK_

EXPECTED VALUES OF POPULATION RISK IN PERSON REM

SFUEL

GROUND INHALED RESUSPD CLOUDSH *INGESTION TOTAL

CS-137 $\quad 1.49 E-20 \quad 1.57 E-23 \quad 6.91 E-23 \quad 2.88 E-25 \quad 5.76 E-20 \quad 7.26 E-20$

TOTALS: $\quad 1.49 \mathrm{E}-20 \quad 1.57 \mathrm{E}-23 \quad 6.91 \mathrm{E}-23 \quad 2.88 \mathrm{E}-25 \quad 5.76 \mathrm{E}-20 \quad 7.26 \mathrm{E}-20$

* NOTE THAT INGESTION RISK IS A SOCIETAL RISK;

THE USER MAY WISH TO TREAT THIS VALUE SEPARATELY. 
RUN DATE: [ 25-MAR-95 AT 14:06:57 ]

ACCIDENT_HAND_CHECK_

EXPECTED RISK VALUES - OTHER

\begin{tabular}{|c|c|c|}
\hline LINK & $\begin{array}{c}E C O N \\
\$ \$ \\
0.00 E+00\end{array}$ & $\begin{array}{l}\text { EARLY } \\
\text { FATALITY } \\
0.00 E+00\end{array}$ \\
\hline & $0.00 E+0$ & 0.0 \\
\hline
\end{tabular}

TOTAL EXPOSED POPULATION: INCIDENT-FREE

TRUCK RURAL

TRUCK SUBURBAN

TRUCK URBAN

\subsection{E+00 PERSONS}

$0.00 E+00$ PERSONS

$0.00 E+00$ PERSONS

TOTAL

9.60E+00 PERSONS

EOI

(PERSONS UNDER PLUME FOOTPRINT FOR A SINGLE ACCIDENT)

TRUCK RURAL

9.18E-03 PERSONS

TRUCK SUBURBAN

1.10E+0O PERSONS

TRUCK URBAN

5.91E+00 PERSONS

END OF RUN 


\section{APPENDIX C}

\section{RISKIND OUTPUT FOR INCIDENT-FREE MAXIMALLY}

EXPOSED INDIVIDUAL SCENARIOS 
C-2 
C.1 T14-PUB.OUT

RADIATION DOSES FOR GENERAL POPULATION TRUCK SCENARIO 
C-4 
\&INDATA

TITLE = ' TRUCK INCIDENT FREE PUBLIC - SNF',

OUTFIL = 'RISKIND. OUT',

IACDT $=0$, IRUTIN $=1$,

FRAD $=1.0,0.0$, TIDX $=21.1$, HSIZE $=4.77$, RSIZE $=0.5048$,

ISTATE $=10, I M O D=1$, IZONE $=3, I R D T Y=3$,

$I A D D=4$

SPEED $=56.3$.

XNAME $=$

- trafFic jam',

' REGULATORY CHECK',

- SERVICE STATION',

' RESIDENT',

XRECEP $=$

$.001,2 * 0$

$.002, \quad 2 \star 0$,

$.020,2 * 0$

$.030,2 \star 0$.

INDR $=1,0,0,1$,

ISHLT $=4,0,0,1$,

TSTP $=0.5,1.0,2.0,0.0$.

\&END 


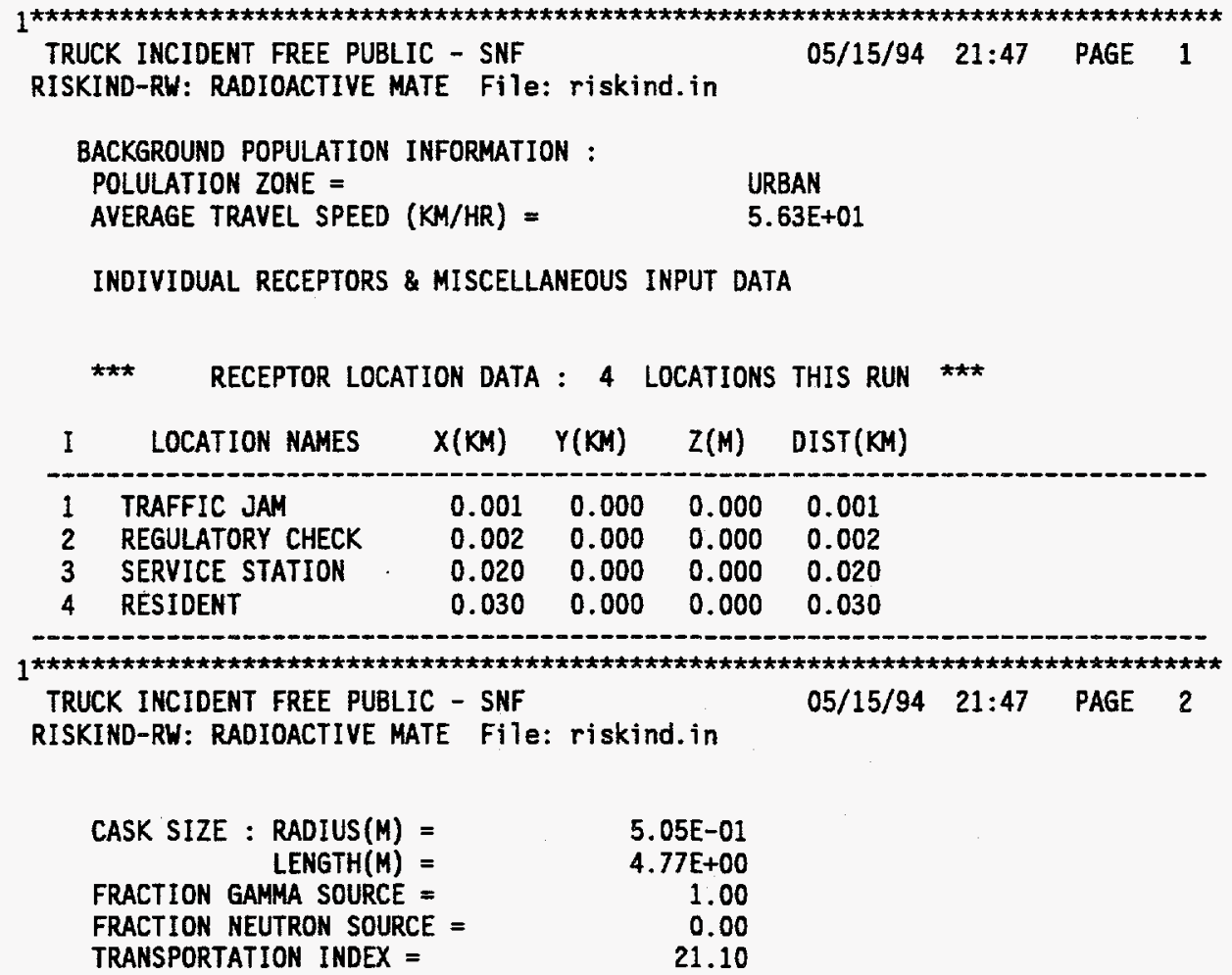

RECEPTOR ROUTINE DOSE FROM EACH SHIPMENT

\begin{tabular}{|c|c|c|c|c|c|}
\hline \multirow[b]{2}{*}{ I } & \multirow[b]{2}{*}{ LOCATION NAMES } & \multirow[b]{2}{*}{ DISTANCE(M) } & \multicolumn{2}{|c|}{$\begin{array}{l}\text { DURING } \\
\text { STOP }\end{array}$} & \multirow{2}{*}{$\begin{array}{l}\text { DURING } \\
\text { TRANSPORT } \\
\text { REM }\end{array}$} \\
\hline & & & HOUR & REM & \\
\hline $\begin{array}{l}1 \\
2 \\
3 \\
4\end{array}$ & $\begin{array}{l}\text { TRAFFIC JAM } \\
\text { REGULATORY CHECK } \\
\text { SERVICE STATION } \\
\text { RESIDENT }\end{array}$ & $\begin{array}{r}1.0 \\
2.0 \\
20.0 \\
30.0\end{array}$ & $\begin{array}{l}0.5 \\
1.0 \\
2.0 \\
0.0\end{array}$ & $\begin{array}{l}5.28 E-03 \\
1.00 E-02 \\
4.07 E-04 \\
0.00 E+00\end{array}$ & $\begin{array}{l}1.06 \mathrm{E}-06 \\
1.56 \mathrm{E}-06 \\
2.02 \mathrm{E}-07 \\
5.02 \mathrm{E}-08\end{array}$ \\
\hline
\end{tabular}




\section{C.2 R14-OCC.OUT}

RADIATION DOSES FOR OCCUPATIONAL RAIL SCENARIO 
\&INDATA

TITLE = ' RAIL INCIDENT FREE OCCUPATIONAL - SNF',

OUTFIL = 'RISKIND.OUT',

IACDT $=0$, IRUTIN $=1$,

FRAD $=1,0,0.0$, TIDX $=21.1$, HSIZE $=5.13$, RSIZE $=1.041$,

ISTATE $=10$, IMOD $=1$, IZONE $=3$,

IADD $=2$,

SPEED $=56.3$.

XNAME $=$

' WORKER',

' REGULATORY CHECK',

XRECEP $=$

$.001,2 * 0$

$.002,2^{\star} 0$.

INDR $=0,0$,

ISHLT $=0,0$,

TSTP $=0.16,1.0$.

\&END 


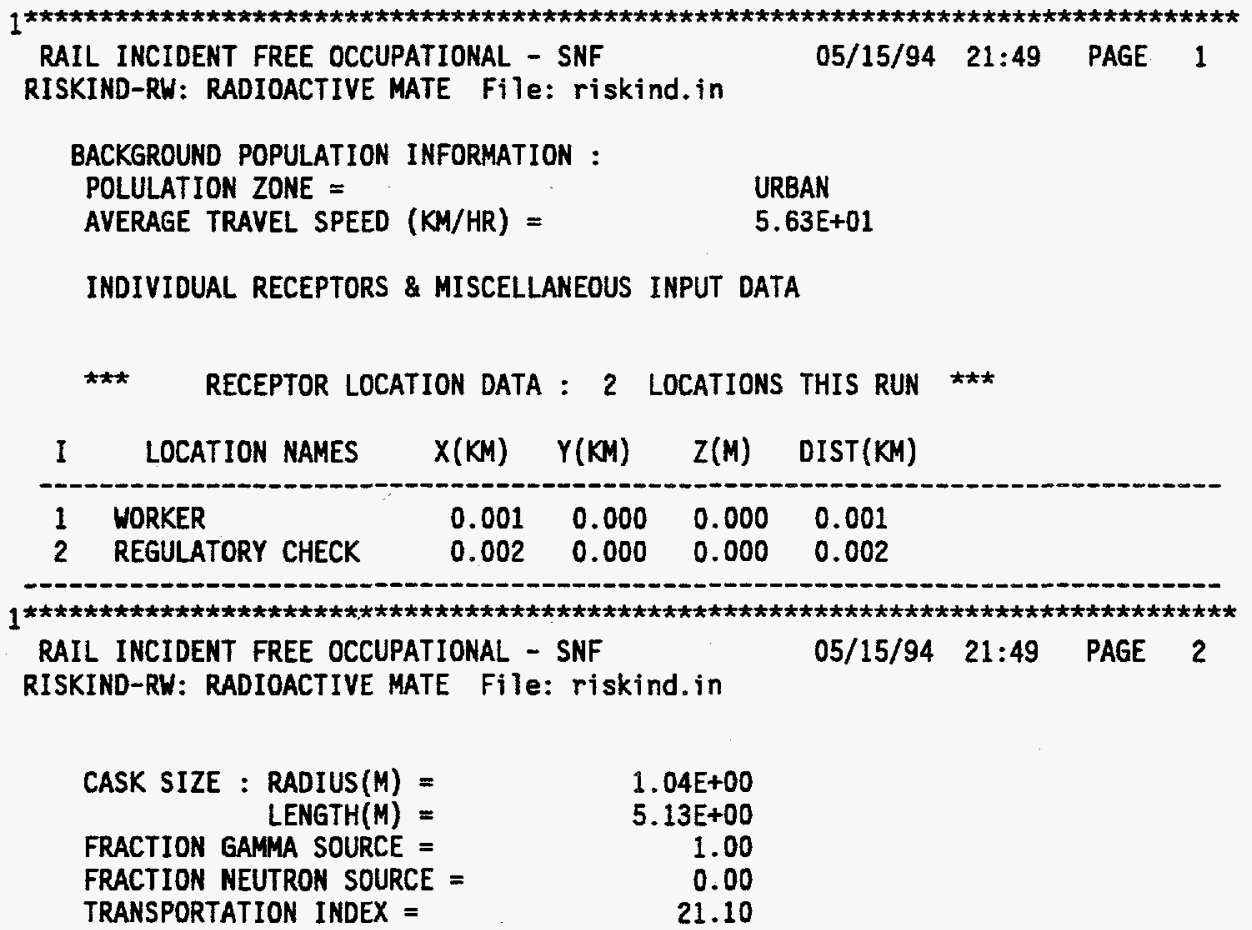

RECEPTOR ROUTINE DOSE FROM EACH SHIPMENT

\begin{tabular}{|c|c|c|c|c|c|}
\hline \multirow[b]{2}{*}{ I } & \multirow[b]{2}{*}{ LOCATION NAMES } & \multirow[b]{2}{*}{ DISTANCE(M) } & \multicolumn{2}{|c|}{$\begin{array}{l}\text { DURING } \\
\text { STOP }\end{array}$} & \multirow{2}{*}{$\begin{array}{l}\text { DURING } \\
\text { TRANSPORT } \\
\text { REM }\end{array}$} \\
\hline & & & HOUR & REM & \\
\hline $\begin{array}{l}1 \\
2\end{array}$ & $\begin{array}{l}\text { WORKER } \\
\text { REGULATORY CHECK }\end{array}$ & $\begin{array}{l}1.0 \\
2.0\end{array}$ & $\begin{array}{l}0.2 \\
1.0\end{array}$ & $\begin{array}{l}3.38 E-03 \\
1.01 E-02\end{array}$ & $\begin{array}{l}2.17 E-06 \\
1.61 E-06\end{array}$ \\
\hline
\end{tabular}


C.3 R14-PUB.OUT

RADIATION DOSES FOR GENERAL POPULATION RAIL SCENARIO 
C-12 
\&INDATA

TITLE $=$ ' RAIL INCIDENT FREE PUBLIC - SNF',

OUTFIL = 'RISKIND.OUT',

IACDT $=0$, IRUTIN $=1$,

FRAD $=1.0,0.0, T I D X=21.1$, HSIZE $=5.13$, RSIZE $=1.041$,

ISTATE $=10$, IMOD $=2$, IZONE $=3$,

$I A D D=4$,

SPEED $=56.3$,

XNAME $=$

' REgulatory check',

- RAILYARD".

' RESIDENT",

' STRANDED CASK',

XRECEP $=$

$.002,2 * 0$,

$.010,2 * 0$

$.030,22^{\star} 0$,

$.200,2 * 0$,

INDR $=0,0,1,1$,

ISHLT $=0,0,4,4$

TSTP $=1.0,2.0,0.0,20.0$

\&END 


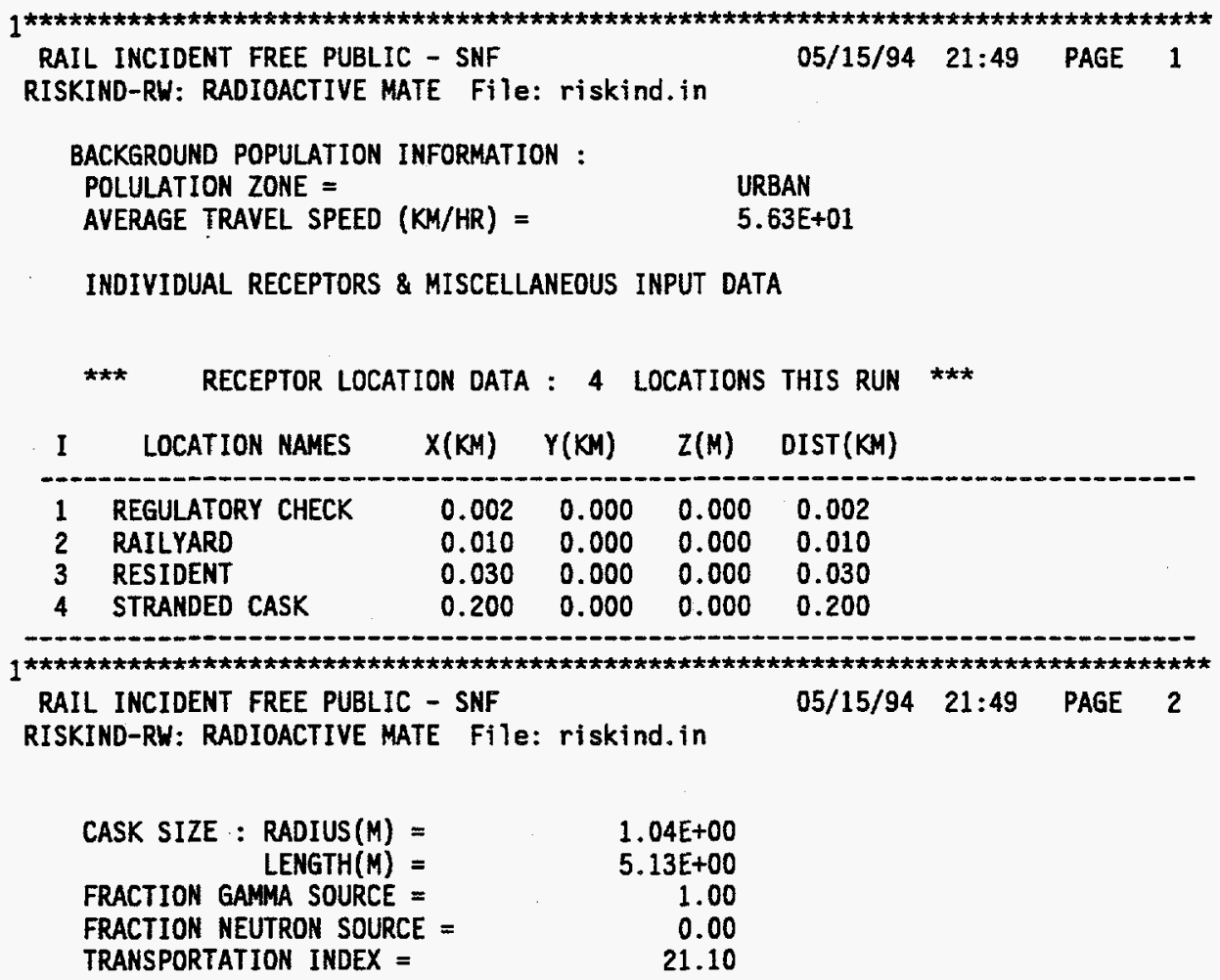

RECEPTOR ROUTINE DOSE FROM EACH SHIPMENT

\begin{tabular}{|c|c|c|c|c|c|}
\hline \multirow[b]{2}{*}{1} & \multirow[b]{2}{*}{ LOCATION NAMES } & \multirow[b]{2}{*}{ DISTANCE $(M)$} & \multicolumn{2}{|c|}{$\begin{array}{l}\text { DURING } \\
\text { STOP }\end{array}$} & \multirow{2}{*}{$\begin{array}{l}\text { DURING } \\
\text { TRANSPORT } \\
\text { REM }\end{array}$} \\
\hline & & & HOUR & REM & \\
\hline $\begin{array}{l}1 \\
2 \\
3 \\
4\end{array}$ & $\begin{array}{l}\text { REGULATORY CHECK } \\
\text { RAILYARD } \\
\text { RESIDENT } \\
\text { STRANDED CASK }\end{array}$ & $\begin{array}{r}2.0 \\
10.0 \\
30.0 \\
200.0\end{array}$ & $\begin{array}{r}1.0 \\
2.0 \\
0.0 \\
20.0\end{array}$ & $\begin{array}{l}1.01 E-02 \\
1.67 E-03 \\
0.00 E+00 \\
1.10 E-05\end{array}$ & $\begin{array}{l}1.61 \mathrm{E}-06 \\
4.72 \mathrm{E}-07 \\
7.17 \mathrm{E}-08 \\
3.71 \mathrm{E}-09\end{array}$ \\
\hline
\end{tabular}




\section{APPENDIX D}

RISKIND OUTPUT FOR TRANSPORTATION ACCIDENT SCENARIO 
D-2 


\section{D.1 VAL.OUT}

RADIATION DOSES FOR ALL EXPOSURE PATHWAYS 
D-4 


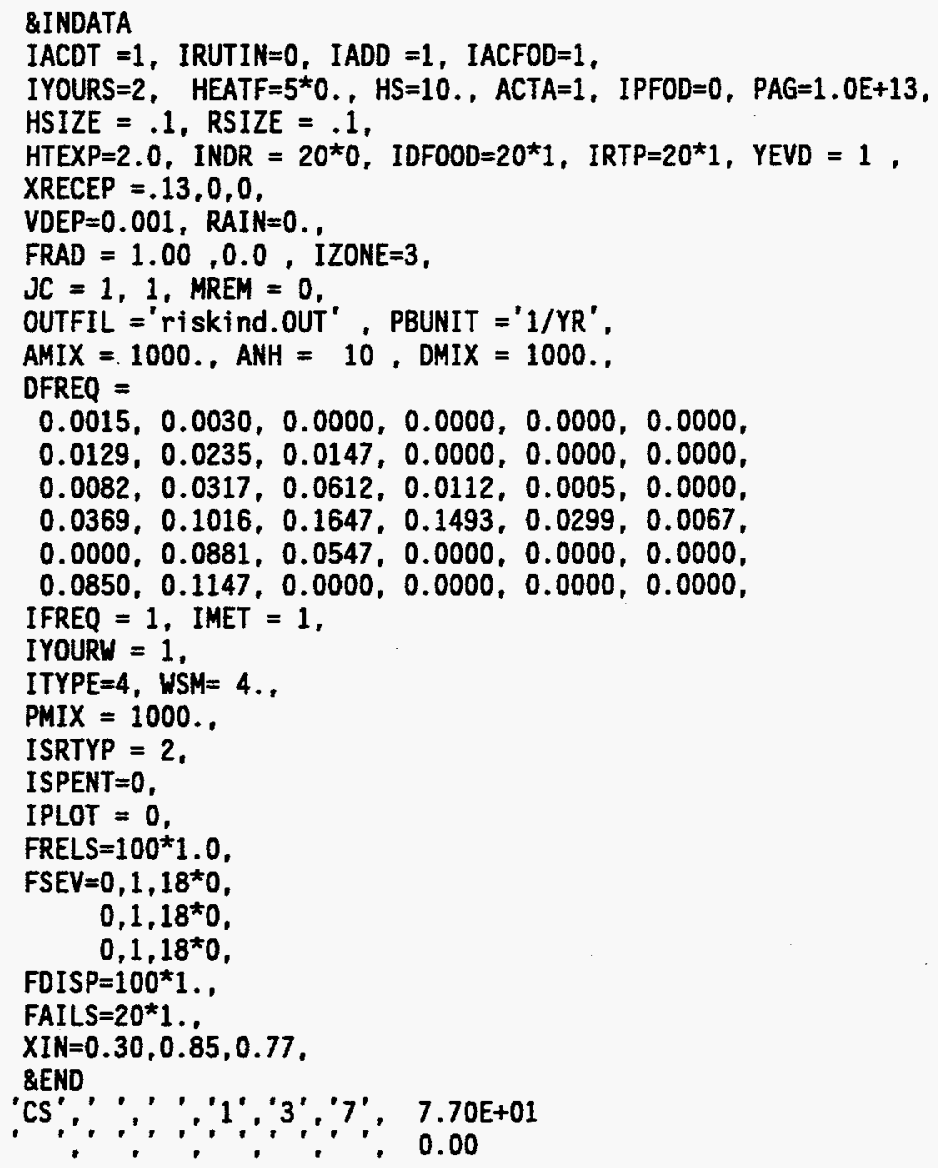




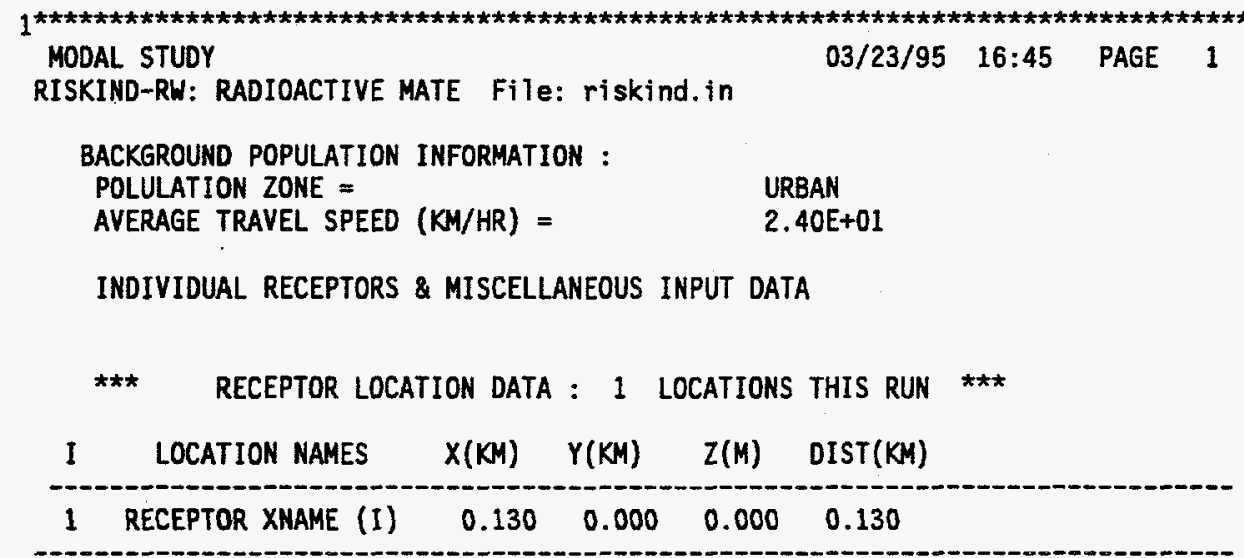

ACCIDENT DATA FROM STATE OF UT

ACCIDENT TRAFFIC FATALITY $(1 / K M)=\quad 0.00 E+00$

ACCIDENT PROBABILITY $(1 / \mathrm{KM})=1.00 \mathrm{E}+00$

INDIVIDUAL DRINKING WATER INTAKE (L/DAY) $=1.00 E+00$

ALL INITIALLY CONTAMINATED FOOD STUFFS ARE DISCARDED

TRANSPORTATION MODE =

TRUCK

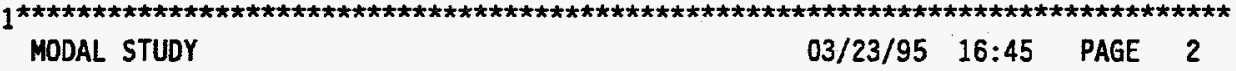

RISKIND-RW: RADIOACTIVE MATE File: riskind.in

FRACTION RELEASED AND ALSO DISPERSED FOR EACH RESPONSE REGION

\begin{tabular}{|c|c|c|c|c|c|c|c|c|c|c|}
\hline NUCLIDE & & & & & ESPONSE & REGION & & & & \\
\hline & 1 & 2 & 3 & 4 & 5 & 6 & 7 & 8 & 9 & 10 \\
\hline $\begin{array}{l}\text { PART. } \\
\text { RU } \\
\text { CS } \\
\text { IODINE } \\
\text { I.GAS }\end{array}$ & $\begin{array}{l}1 . E+\infty 0 \\
1 . E+00 \\
1 . E+\infty 0 \\
1 . E+\infty 0 \\
1 . E+\infty 0\end{array}$ & $\begin{array}{l}1 . E+00 \\
1 . E+00 \\
1 . E+00 \\
1 . E+00 \\
1 . E+00\end{array}$ & $\begin{array}{l}1 \cdot E+00 \\
1 . E+00 \\
1 \cdot E+00 \\
1 . E+00 \\
1 \cdot E+00\end{array}$ & $\begin{array}{l}\text { 1. } E+00 \\
1 . E+00 \\
1 . E+00 \\
1 . E+00 \\
1 . E+00\end{array}$ & $\begin{array}{l}1 . E+00 \\
1 . E+00 \\
1 . E+00 \\
1 . E+00 \\
1 . E+00\end{array}$ & $\begin{array}{l}\text { 1. } E+\infty 0 \\
1 . E+00 \\
1 . E+\infty 0 \\
1 . E+\infty 0 \\
1 . E+00\end{array}$ & $\begin{array}{l}1 . E+00 \\
1 . E+00 \\
1 . E+00 \\
1 . E+00 \\
1 . E+00\end{array}$ & $\begin{array}{l}1 . E+00 \\
1 . E+00 \\
1 . E+00 \\
1 . E+00 \\
1 . E+00\end{array}$ & $\begin{array}{l}1 . E+00 \\
1 . E+00 \\
1 . E+00 \\
1 . E+00 \\
1 . E+00\end{array}$ & $\begin{array}{l}1 . E+00 \\
1 . E+00 \\
1 . E+00 \\
1 . E+00 \\
1 . E+00\end{array}$ \\
\hline PROB & $0 . E+00$ & 1. $E+00$ & $0 . E+00$ & $0 . E+00$ & $0 . E+00$ & $0 . E+\infty 0$ & $0 . E+\infty$ & $0 . E+00$ & $0 . E+00$ & $0 . E+00$ \\
\hline
\end{tabular}

FRACTION RELEASED AND ALSO OISPERSED FOR EACH RESPONSE REGION

NUCLIDE

RESPONSE REGION

\begin{tabular}{|c|c|c|c|c|c|c|c|c|c|c|}
\hline TYPE & & & & & & & & & & \\
\hline & 11 & 12 & 13 & 14 & 15 & 16 & 17 & 18 & 19 & 20 \\
\hline $\begin{array}{l}\text { RU } \\
\text { CS } \\
\text { IODINE } \\
\text { I.GAS }\end{array}$ & $\begin{array}{l}1 . E+\infty O \\
1 . E+O D \\
1 . E+00 \\
1 . E+00 \\
1 . E+00\end{array}$ & $\begin{array}{l}1 \cdot E+00 \\
1 \cdot E+00 \\
1 \cdot E+00 \\
1 \cdot E+00 \\
1 \cdot E+00\end{array}$ & $\begin{array}{l}\text { 1. } E+00 \\
1 . E+00 \\
1 . E+00 \\
1 . E+00 \\
1 . E+00\end{array}$ & $\begin{array}{l}\text { 1. } E+00 \\
1 . E+00 \\
1 . E+00 \\
1 . E+00 \\
1 . E+00\end{array}$ & $\begin{array}{l}\text { 1. } E+00 \\
1 . E+00 \\
1 . E+00 \\
1 . E+00 \\
1 . E+00\end{array}$ & $\begin{array}{l}1 . E+00 \\
1 . E+00 \\
1 . E+00 \\
1 . E+00 \\
1 . E+00\end{array}$ & $\begin{array}{l}1 . E+00 \\
1 . E+00 \\
1 . E+00 \\
1 . E+00 \\
1 . E+00\end{array}$ & $\begin{array}{l}1 . E+00 \\
1 . E+00 \\
1 \cdot E+00 \\
1 . E+00 \\
1 . E+00\end{array}$ & $\begin{array}{l}1 . E+00 \\
1 . E+00 \\
1 . E+00 \\
1 . E+00 \\
1 . E+00\end{array}$ & $\begin{array}{l}1 . E+00 \\
1 . E+00 \\
1 . E+00 \\
1 . E+00 \\
1 . E+00\end{array}$ \\
\hline & $\mathrm{F}+0 \mathrm{O}$ & & r. & & $0 . F+00$ & a. $E+\infty 0$ & $0 . E+0$ & $0 . F+00$ & $0 . E+00$ & $0 . E+\infty 0$ \\
\hline
\end{tabular}




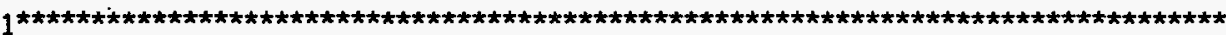

MODAL STUDY

RISKIND-RW: RADIOACTIVE MATE File: riskind.in

EXTERNAL DOSE RATE MULTIPLIER FOR EACH ACCIDENT RESPONSE REGION (MULTIPLIER OF TI)

\begin{tabular}{|c|c|c|c|c|c|c|c|c|c|c|}
\hline \multirow[t]{2}{*}{ TYPE } & \multicolumn{9}{|c|}{$\begin{array}{l}\text { RESPONSE REGION } \\
\text { REN }\end{array}$} & \multirow[b]{2}{*}{10} \\
\hline & 1 & 2 & 3 & 4 & 5 & 6 & 7 & 8 & 9 & \\
\hline $\begin{array}{l}\text { GAMMA } \\
\text { NUTRN }\end{array}$ & $\begin{array}{l}1.00 \\
1.00\end{array}$ & $\begin{array}{l}2.00 \\
2.00\end{array}$ & $\begin{array}{l}2.00 \\
2.00\end{array}$ & $\begin{array}{l}2.00 \\
2.00\end{array}$ & $\begin{array}{l}2.00 \\
2.00\end{array}$ & $\begin{array}{l}2.00 \\
2.00\end{array}$ & $\begin{array}{l}2.00 \\
2.00\end{array}$ & $\begin{array}{l}2.00 \\
2.00\end{array}$ & $\begin{array}{l}2.00 \\
2.00\end{array}$ & $\begin{array}{l}2.00 \\
2.00\end{array}$ \\
\hline
\end{tabular}

EXTERNAL DOSE RATE MULTIPLIER FOR EACH ACCIDENT RESPONSE REGION

(MULTIPLIER OF TI)

TYPE

\begin{tabular}{|c|c|c|c|c|c|c|c|c|c|c|}
\hline IITE & 11 & 12 & 13 & 14 & 15 & 16 & 17 & 18 & 19 & 20 \\
\hline $\begin{array}{l}\text { GAMMA } \\
\text { NUTRN }\end{array}$ & $\begin{array}{l}2.00 \\
2.00\end{array}$ & $\begin{array}{l}2.00 \\
2.00\end{array}$ & $\begin{array}{l}2.00 \\
2.00\end{array}$ & $\begin{array}{l}2.00 \\
2.00\end{array}$ & $\begin{array}{l}2.00 \\
2.00\end{array}$ & $\begin{array}{l}2.00 \\
2.00\end{array}$ & $\begin{array}{l}2.00 \\
2.00\end{array}$ & $\begin{array}{l}2.00 \\
2.00\end{array}$ & $\begin{array}{l}2.00 \\
2.00\end{array}$ & $\begin{array}{l}2.00 \\
2.00\end{array}$ \\
\hline
\end{tabular}

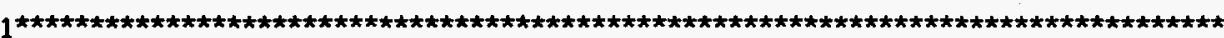

MODAL STUDY

RISKIND-RW: RADIOACTIVE MATE File: riskind.in

$03 / 23 / 95$ 16:45 PAGE 4

$\begin{array}{rr}\text { CASK SIZE : RADIUS }(M)= & 1.00 E-01 \\ \text { LENGTH }(M)= & 1.00 E-01 \\ \text { FRACTION GAMMA SOURCE }= & 1.00 \\ \text { FRACTION NEUTRON SOURCE }= & 0.00 \\ \text { TRAMSPORTATION INDEX }= & 10.00\end{array}$

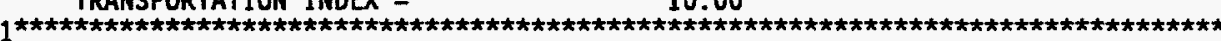

MODAL STUDY

RISKIND-RW: RADIOACTIVE MATE File: riskind.in

03/23/95 16:45 PAGE 5

ACCIDENT SEVERITY NUMBER $=$
ACCIDENT SCENARIO PROBABILITY, $1 / \mathrm{YR}^{2}=1.00 E+00$

\begin{tabular}{|c|c|c|c|c|c|c|c|}
\hline NUCLIDE & $\operatorname{DCAY}(1 / Y R)$ & CURIES & \multicolumn{5}{|c|}{ RELATIVE HAZARD } \\
\hline & & & PLUME & INGESTION & G. & INE & \\
\hline CS 137 & $2.30 E-02$ & $7.70 E+01$ & $1.00 E+00$ & $1.00 E+00$ & & $E+00$ & \\
\hline 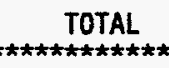 & & & 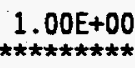 & 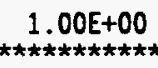 & & $\begin{array}{l}E+00 \\
\star \star \star \star \star \star\end{array}$ & \\
\hline MODAL STUDY & & & & $03 / 23 / 95$ & $6: 45$ & PAGE & 6 \\
\hline
\end{tabular}

RISKIND-RW: RADIOACTIVE MATE File: riskind.in

\begin{tabular}{lccc} 
NUCLIDE & INVENTORY(Ci) & FRAC-REL & Ci-REL \\
\hline CS 137 & $7.70 E+01$ & $1.00 E+00$ & $7.70 E+01$
\end{tabular}

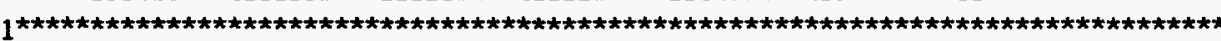

MODAL STUDY $\quad 03 / 23 / 95 \quad 16: 45$ PAGE 7

RISKIND-RW: RADIOACTIVE MATE File: riskind.in

ONE YEAR COMMITTED ACUTE INHALATION DOSE CONVERSION FACTORS

(RAD/SEC/PCI/M3)

\begin{tabular}{lccccc} 
NUCLIDE & R.MARROW & \multicolumn{1}{c}{ LUNG } & GI-LLI & EFFECTIVE & GONADS \\
\hline CS137 & $9.80 E-12$ & $1.05 E-11$ & $1.05 E-11$ & $1.02 E-11$ & $9.45 E-12$
\end{tabular}

50 YEAR CHRONIC INHALATION DOSE COMMITMENT FACTORS (REM/YR/PCI/M3)

NUCLIDE R.MARROW LUNG GI-LLI EFFECTIVE GONADS 


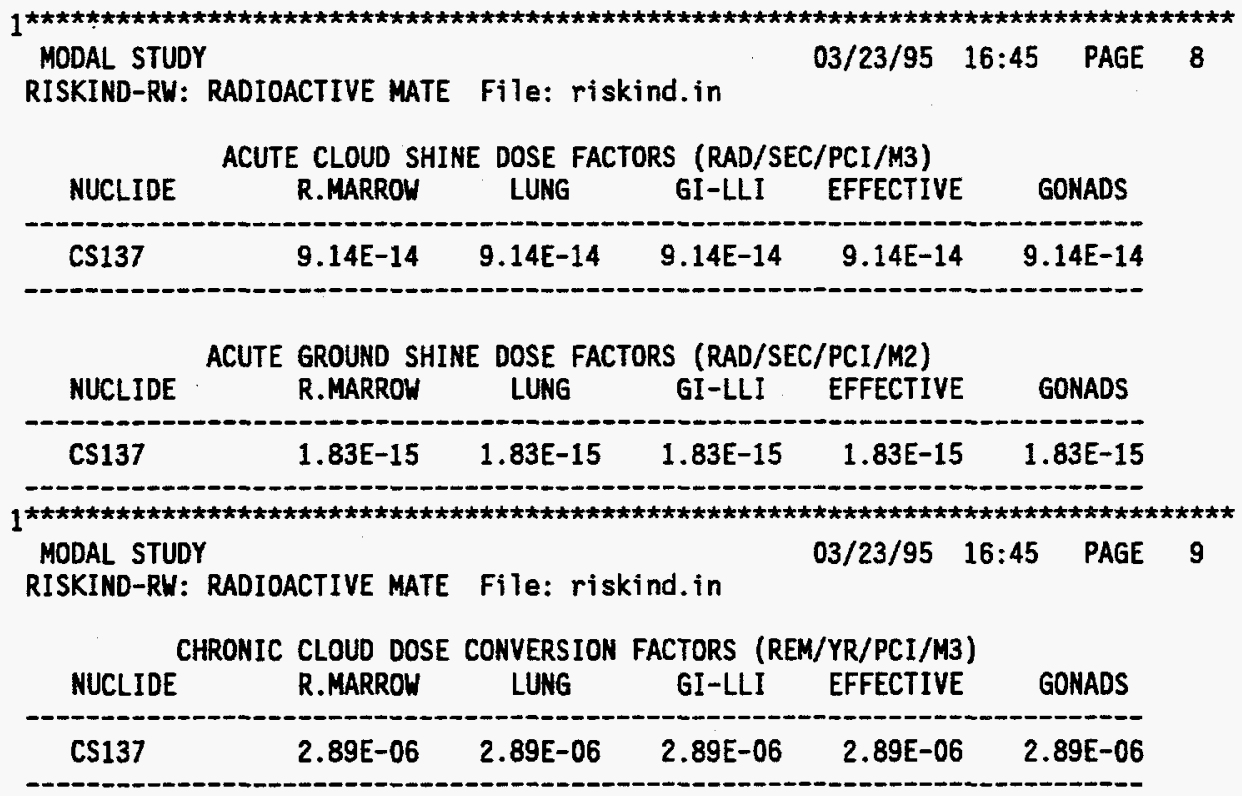

CHRONIC GROUND DOSE CONVERSION FACTORS (REM/YR/PCI/M2)

\begin{tabular}{|c|c|c|c|c|c|}
\hline NUCLIDE & R.MARROW & LUNG & GI-LLI & EFFECTIVE & GONADS \\
\hline CS137 & $5.76 E-08$ & $5.76 E-08$ & $5.76 E-08$ & $5.76 E-08$ & $5.76 \mathrm{E}-08$ \\
\hline
\end{tabular}

\begin{tabular}{cccccr} 
NUCLIDE & $\begin{array}{c}\text { INGESTION DOSE CONVERSION FACTORS (REM/PCI) } \\
\text { R.MARROW }\end{array}$ & LUNG & GI-LLI & EFFECTIVE & GONADS \\
\hline CS137 & $4.90 E-08$ & $4.72 E-08$ & $5.30 E-08$ & $5.02 E-08$ & $5.16 E-08$ \\
\hline
\end{tabular}

DISPERSION CALCULATION PARAMETERS (PUFIND):

$\begin{array}{lc}\text { PHYSICAL RELEASE HEIGHT = } & 10.00(\mathrm{M}) \\ \text { SOURCE EFFECTIVE SIZE }= & 0.14(\mathrm{M}) \\ \text { ANAMETER HEIGHT }= & 10.00(\mathrm{M}) \\ \text { AMBIENT TEMPERATURE }= & 288.15(\mathrm{~K}) \\ \text { DRY DEPOSITION VELOCITY }= & 0.0010(\mathrm{M} / \mathrm{S}) \\ \text { DEPOSITION VEL OVER VATER }= & 0.010(\mathrm{M} / \mathrm{S}) \\ \text { DEPOSITION VEL OVER VEGETATION }= & 0.010(\mathrm{M} / \mathrm{S}) \\ \text { STABILITY CLASS }= & 4 \\ \text { WIND SPEED }= & 4.00(\mathrm{M} / \mathrm{S}) \\ \text { SOURCE TEMPERATURE }= & 0.00(\mathrm{~K}) \\ \text { HEAT FLUX }= & 0.00(\mathrm{CAL} / \mathrm{SEC}) \\ \text { FINAL EFFECTIVE RELEASE HEIGHT }= & 10.00(\mathrm{M})\end{array}$

SUMMARY OF RECEPTOR CONCENTRATION AND DEPLETION FACTORS

\begin{tabular}{|c|c|c|c|c|c|c|}
\hline RCPTR & $\operatorname{DIST}(K M)$ & ANGLE & $\begin{array}{r}X 0 Q(G A S) \\
\left(S E C / M^{\star \star} 3\right)\end{array}$ & 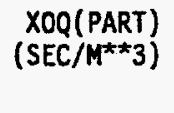 & $\begin{array}{l}\text { DEPLTION } \\
\text { FRACTION }\end{array}$ & $\begin{array}{l}\text { DEPOSITION } \\
\left(1 / M^{* *} 2\right)\end{array}$ \\
\hline 1 & $1.30 \mathrm{E}-01$ & $0.00 E+00$ & 2.64E-04 & $2.63 E-04$ & $2.55 \mathrm{E}-04$ & $2.63 E-07$ \\
\hline
\end{tabular}


1 1

MODAL STUDY

03/23/95 16:45 PAGE 10

RISKIND-RW: RADIOACTIVE MATE File: riskind.in

SUMMARY OF RECEPTOR POTENTIAL DOSE CONSEQUENCES

FATALITY AND GENETIC RISKS

I LOCATION NAMES

\begin{tabular}{llcc}
\multicolumn{2}{c}{ ACUTE } & \multicolumn{2}{c}{ LONG TERM } \\
\hline EDE & CHANCE & EDE & CHANCE \\
REM & FATALITY & REM & FATALITY GENETIC.E
\end{tabular}

1 RECEPTOR XNAME (I) $\quad 2.29 E-01 \quad 0.00 E+00 \quad 1.97 E+00 \quad 9.84 E-04 \quad 2.56 E-04$

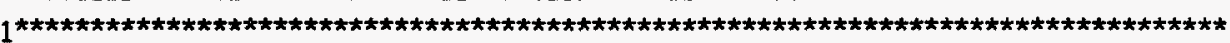

MODAL STUDY

RISKIND-RW: RADIOACTIVE MATE File: riskind.in

03/23/95 16:45 PAGE 11

TIME-INTEGRATED( 1 YEARS) DOSE CONSEQUENCE (REM)

RECEPTOR 1 : RECEPTOR XNAME (I)

DOWN WIND DIST $(\mathrm{KM})=1.30 \mathrm{E}-01$

CROSS WIND DIST $(\mathrm{KM})=0.00 E+00$

\begin{tabular}{lccccc} 
PATHWAY & R.MARROW & LUNG & GI-LLI & EFFECTIVE & GONADS \\
\hline PLUME & $2.21 E-01$ & $2.34 E-01$ & $2.36 E-01$ & $2.29 E-01$ & $2.33 E-01$ \\
EXT CASK & $2.42 E-07$ & $2.42 E-07$ & $2.42 E-07$ & $2.42 E-07$ & $2.42 E-07$ \\
RESUSPENSN. & $7.31 E-03$ & $7.76 E-03$ & $7.84 E-03$ & $7.60 E-03$ & $7.72 E-03$ \\
INGESTION & $1.19 E+00$ & $1.14 E+00$ & $1.28 E+00$ & $1.22 E+00$ & $1.25 E+00$ \\
EXT GROUND & $5.16 E-01$ & $5.16 E-01$ & $5.16 E-01$ & $5.16 E-01$ & $5.16 E-01$ \\
EXT CLOUD & $7.99 E-05$ & $7.99 E-05$ & $7.99 E-05$ & $7.99 E-05$ & $7.99 E-05$ \\
WATER & $0.00 E+00$ & $0.00 E+00$ & $0.00 E+00$ & $0.00 E+00$ & $0.00 E+00$ \\
\hline TOTAL & $1.93 E+00$ & $1.90 E+00$ & $2.04 E+00$ & $1.97 E+00$ & $2.01 E+00$
\end{tabular}

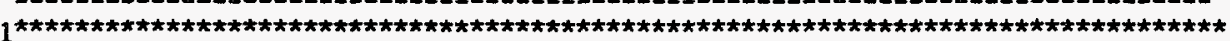

MODAL STUDY

RISKIND-RW: RADIOACTIVE MATE File: riskind.in

03/23/95 16:45 PAGE 12

SUMMARY OF PROBABILITY WEIGHTED RISKS

RECEPTOR 1 : RECEPTOR XNAME (I)

DOWN WIND DIST $(\mathrm{KM})=1.30 \mathrm{E}-01$

CROSS WIND DIST $(K M)=0.00 E+00$

\begin{tabular}{|c|c|c|c|c|}
\hline & \multicolumn{2}{|c|}{ INCIDENT FREE } & \multicolumn{2}{|c|}{ ACCIDENTS } \\
\hline & $\begin{array}{l}\text { TRANSIT } \\
1 / \text { CASK }\end{array}$ & $\begin{array}{l}\text { STOP } \\
1 / \text { STOP }\end{array}$ & $\begin{array}{c}\text { AIR/GROUND } \\
\text { 1/EVENT }\end{array}$ & $\begin{array}{l}\text { WATER } \\
\text { 1/EVENT }\end{array}$ \\
\hline $\begin{array}{l}\text { EFFECTIVE DOSE } \\
\text { (REM) }\end{array}$ & $0.00 E+00$ & $0.00 E+00$ & $1.97 \mathrm{E}+00$ & $0.00 E+00$ \\
\hline LATENT CANCER & & & & \\
\hline $\begin{array}{l}\text { MORTALITY } \\
\text { (CHANCE) }\end{array}$ & $0.00 \mathrm{E}+00$ & $0.00 E+00$ & $9.84 E-04$ & $0.00 E+00$ \\
\hline $\begin{array}{l}\text { GENETIC EFFECT } \\
\text { (CHANCE) }\end{array}$ & $0.00 \mathrm{E}+00$ & $0.00 E+00$ & $2.56 E-04$ & $0.00 E+00$ \\
\hline $\begin{array}{l}\text { ACUT'E MORTALITY } \\
\text { (CHANCE) }\end{array}$ & & & $0.00 E+00$ & \\
\hline
\end{tabular}




\section{D.2 VAL-V.OUT}

RADIATION DOSES INCLUDING ONLY VEGETABLE INGESTION PATHWAY 
D-12 


\section{\&INDATA}

IACDT $=1$, IRUTIN=0, IADD $=1$, IACFOD $=1$,

IYOURS $=2, H E A T F=5 * 0 ., H S=10 ., A C T A=1, I P F O D=0, P A G=1.0 E+13$,

HSIZE $=.1$, RSIZE $=.1$.

HTEXP=2.0, INDR $=20 * 0, I D F O O D=20 * 1, I R T P=20 \star 1$, YEVD $=1$,

XRECEP $=.13,0,0$

VDEP $=0.001, R A I N=0$. ,

FRAD $=1.00,0.0$, IZONE $=3$.

$\mathrm{JC}=1,1, \operatorname{MREM}=0$,

OUTFIL $=$ 'riskind.OUT', PBUNIT $=$ '1/YR',

AMIX $=1000 .$, ANH $=10$, DMIX $=1000$.

DFREQ $=$

$0.0015,0.0030,0.0000,0.0000,0.0000,0.0000$

$0.0129,0.0235,0.0147,0.0000,0.0000,0.0000$.

$0.0082,0.0317,0.0612,0.0112,0.0005,0.0000$,

$0.0369,0.1016,0.1647,0.1493,0.0299,0.0067$,

$0.0000,0.0881,0.0547,0.0000,0.0000,0.0000$,

$0.0850,0.1147,0.0000,0.0000,0.0000,0.0000$.

IFREQ $=1$. IMET $=1$.

IYOURW $=1$,

ITYPE $=4$, WSH $=4$.

PMIX $=1000$.

ISRTYP $=2$,

ISPENT $=0$,

IPLOT $=0$

FRELS $=100 * 1.0$,

FSEV $=0,1,18 * 0$ $0,1,18 * 0$ $0,1,18^{\star} 0$,

FDISP $=100 * 1$,

FAILS $=20 * 1$.

$X I N=0.00,0.00,0.77$,

\&END

'CS', $\because 1{ }^{\prime}, 3^{\prime}, 7^{\prime}, 7.70 \mathrm{E}+01$ 


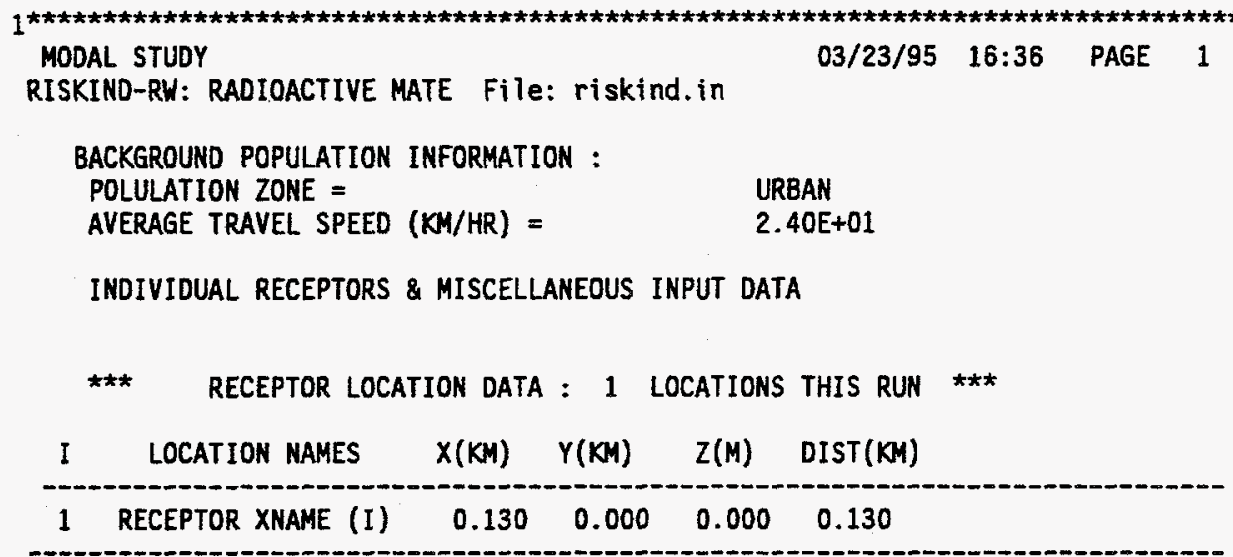

ACCIDENT DATA FROM STATE OF UT ACCIDENT TRAFFIC FATALITY $(1 / K M)=0.00 E+00$ ACCIDENT PROBABILITY $(1 / K M)=1.00 E+00$ INDIVIDUAL DRINKING WATER INTAKE $(L / D A Y)=1.00 E+00$ ALL INITIALLY CONTAMINATED FOOD STUFFS ARE DISCARDED

TRANSPORTATION MODE = TRUCK

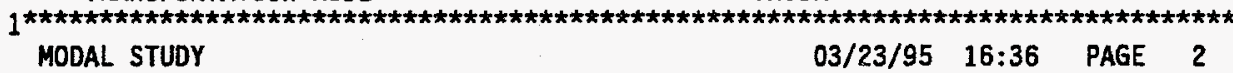
RISKIND-RW: RADIOACTIVE MATE File: riskind.in

FRACTION RELEASED AND ALSO DISPERSED FOR EACH RESPONSE REGION

\begin{tabular}{|c|c|c|c|c|c|c|c|c|c|c|}
\hline \multirow{2}{*}{$\begin{array}{l}\text { NUCLIDE } \\
\text { TYPE }\end{array}$} & \multicolumn{10}{|c|}{ RESPONSE REGION } \\
\hline & 1 & 2 & 3 & 4 & 5 & 6 & 7 & 8 & 9 & 10 \\
\hline $\begin{array}{l}\text { PART. } \\
\text { RU } \\
\text { CS } \\
\text { IODINE } \\
\text { I.GAS }\end{array}$ & $\begin{array}{l}\text { 1.E+O0 } \\
1 . E+00 \\
1 . E+00 \\
1 . E+\infty 0 \\
1 . E+00\end{array}$ & $\begin{array}{l}1 . E+00 \\
1 . E+00 \\
1 . E+00 \\
1 . E+00 \\
1 . E+00\end{array}$ & $\begin{array}{l}1 . E+00 \\
1 . E+00 \\
1 . E+00 \\
1 . E+00 \\
1 . E+00\end{array}$ & $\begin{array}{l}1 . E+00 \\
1 . E+00 \\
1 . E+00 \\
1 . E+00 \\
1 . E+00\end{array}$ & $\begin{array}{l}1 . E+\infty 0 \\
1 . E+00 \\
1 . E+\infty 0 \\
1 . E+\infty 0 \\
1 . E+00\end{array}$ & $\begin{array}{l}1 . E+\infty 0 \\
1 . E+\infty 0 \\
1 . E+\infty 0 \\
1 . E+00 \\
1 . E+00\end{array}$ & $\begin{array}{l}1 . E+00 \\
1 . E+00 \\
1 . E+00 \\
1 . E+00 \\
1 . E+00\end{array}$ & $\begin{array}{l}1 . E+00 \\
1 . E+00 \\
1 . E+00 \\
1 . E+00 \\
1 . E+00\end{array}$ & $\begin{array}{l}1 . E+00 \\
1 . E+00 \\
1 . E+00 \\
1 . E+00 \\
1 . E+00\end{array}$ & $\begin{array}{l}\text { 1. } E+\infty 0 \\
1 . E+\infty 0 \\
1 . E+00 \\
1 . E+00 \\
1 . E+00\end{array}$ \\
\hline PROB & $0 . E+00$ & 1. $E+00$ & $0 . E+00$ & $0 . E+00$ & $0 . E+D 0$ & O. E+00 & D. E+DO & $0 . E+00$ & $0 . E+00$ & $0 . E+00$ \\
\hline
\end{tabular}

FRACTION RELEASED AND ALSO DISPERSED FOR EACH RESPONSE REGION

\begin{tabular}{|c|c|c|c|c|c|c|c|c|c|c|}
\hline \multirow{2}{*}{$\begin{array}{l}\text { NUCLIDE } \\
\text { TYPE }\end{array}$} & \multicolumn{10}{|c|}{ RESPONSE REGION } \\
\hline & 11 & 12 & 13 & 14 & 15 & 16 & 17 & 18 & 19 & 20 \\
\hline $\begin{array}{l}\text { PART. } \\
\text { RU } \\
\text { CS } \\
\text { IODINE } \\
\text { I.GAS }\end{array}$ & $\begin{array}{l}\text { 1. } E+00 \\
1 . E+00 \\
\text { 1. E+00 } \\
\text { 1. E+00 } \\
\text { 1. E+00 }\end{array}$ & $\begin{array}{l}\text { 1. } E+\infty 0 \\
\text { 1. } E+\infty 0 \\
1 . E+\infty 0 \\
1 . E+\infty 0 \\
1 . E+\infty 0\end{array}$ & $\begin{array}{l}\text { 1. } E+00 \\
\text { 1. } E+\infty 0 \\
1 . E+00 \\
\text { 1. } E+00 \\
1 . E+\infty 0\end{array}$ & $\begin{array}{l}1 . E+00 \\
1 . E+00 \\
1 . E+00 \\
1 . E+00 \\
1 . E+00\end{array}$ & $\begin{array}{l}1 . E+\infty 0 \\
1 . E+\infty 0 \\
1 . E+\infty 0 \\
1 . E+\infty 0 \\
1 . E+\infty 0\end{array}$ & $\begin{array}{l}\text { 1. } E+\infty 0 \\
1 . E+\infty 0 \\
1 . E+\infty 0 \\
1 . E+\infty 0 \\
1 . E+\infty 0\end{array}$ & $\begin{array}{l}\text { 1. } E+\infty 0 \\
1 . E+\infty 0 \\
1 . E+00 \\
1 . E+\infty 0 \\
1 . E+\infty 0\end{array}$ & $\begin{array}{l}1 . E+00 \\
1 . E+00 \\
1 . E+00 \\
1 . E+00 \\
1 . E+00\end{array}$ & $\begin{array}{l}1 . E+00 \\
1 . E+00 \\
1 . E+00 \\
1 . E+00 \\
1 . E+00\end{array}$ & $\begin{array}{l}1 . E+\infty 0 \\
1 . E+\infty 0 \\
1 . E+\infty 0 \\
1 . E+\infty 0 \\
1 . E+\infty 0\end{array}$ \\
\hline PROB & $0 . E+\infty 0$ & $0 . E+00$ & $0 . E+00$ & $0 . E+00$ & $0 . E+\infty 0$ & $0 . E+O O$ & $0 . E+00$ & $0 . E+00$ & $0 . E+00$ & $0 . E+00$ \\
\hline
\end{tabular}


1 1

MODAL STUDY

RISKIND-RW: RADIOACTIVE MATE File: riskind.in

EXTERNAL DOSE RATE MULTIPLIER FOR EACH ACCIDENT RESPONSE REGION (MULTIPLIER OF TI)

\begin{tabular}{|c|c|c|c|c|c|c|c|c|c|c|}
\hline \multirow[t]{2}{*}{ TYPE } & \multicolumn{10}{|c|}{ RESPONSE REGION } \\
\hline & 1 & 2 & 3 & 4 & 5 & 6 & 7 & 8 & 9 & 10 \\
\hline $\begin{array}{l}\text { GAMNA } \\
\text { NUTRN }\end{array}$ & $\begin{array}{l}1.00 \\
1.00\end{array}$ & $\begin{array}{l}2.00 \\
2.00\end{array}$ & $\begin{array}{l}2.00 \\
2.00\end{array}$ & $\begin{array}{l}2.00 \\
2.00\end{array}$ & $\begin{array}{l}2.00 \\
2.00\end{array}$ & $\begin{array}{l}2.00 \\
2.00\end{array}$ & $\begin{array}{l}2.00 \\
2.00\end{array}$ & $\begin{array}{l}2.00 \\
2.00\end{array}$ & $\begin{array}{l}2.00 \\
2.00\end{array}$ & $\begin{array}{l}2.00 \\
2.00\end{array}$ \\
\hline
\end{tabular}

EXTERNAL DOSE RATE MULTIPLIER FOR EACH ACCIDENT RESPONSE REGION (MULTIPLIER OF TI)

\begin{tabular}{|c|c|c|c|c|c|c|c|c|c|c|}
\hline \multirow[t]{2}{*}{ TYPE } & \multicolumn{10}{|c|}{ RESPONSE REGION } \\
\hline & 11 & 12 & 13 & 14 & 15 & 16 & 17 & 18 & 19 & 20 \\
\hline $\begin{array}{l}\text { GAMMA } \\
\text { NUTRN }\end{array}$ & $\begin{array}{l}2.00 \\
2.00\end{array}$ & $\begin{array}{l}2.00 \\
2.00\end{array}$ & $\begin{array}{l}2.00 \\
2.00\end{array}$ & $\begin{array}{l}2.00 \\
2.00\end{array}$ & $\begin{array}{l}2.0 \\
2.0\end{array}$ & $\begin{array}{l}2.0 \\
2.0\end{array}$ & $\begin{array}{l}2.00 \\
2.00\end{array}$ & $\begin{array}{l}2.00 \\
2.00\end{array}$ & $\begin{array}{l}2.00 \\
2.00\end{array}$ & $\begin{array}{l}2.00 \\
2.00\end{array}$ \\
\hline
\end{tabular}

1 1

MODAL STUDY

RISKIND-RH: RADIOACTIVE MATE File: riskind.in

03/23/95 16:36 PAGE 4

$\begin{array}{rr}\text { CASK SIZE : RADIUS }(M)= & 1.00 E-01 \\ \text { LENGTH }(M)= & 1.00 E-01 \\ \text { FRACTION GAMMA SOURCE }= & 1.00 \\ \text { FRACTION NEUTRON SOURCE } & 0.00 \\ \text { TRANSPORTATION INDEX }= & 10.00\end{array}$

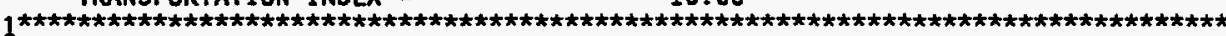

MODAL STUDY $\quad 03 / 23 / 95 \quad 16: 36$ PAGE 5

RISKIND-RW: RADIOACTIVE MATE File: riskind.in

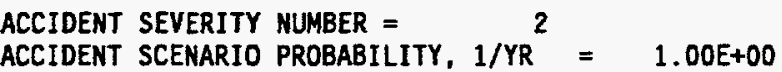

\begin{tabular}{|c|c|c|c|c|c|}
\hline \multirow[t]{2}{*}{ NUCLIDE } & \multirow[t]{2}{*}{$\operatorname{DCAY}(1 / Y R)$} & \multirow[t]{2}{*}{ CURIES } & \multicolumn{3}{|c|}{ RELATIVE HAZARD } \\
\hline & & & PLUME & INGESTION & G.SHINE \\
\hline CS 137 & $2.30 \mathrm{E}-02$ & $7.70 \mathrm{E}+01$ & $1.00 \mathrm{E}+00$ & $1.00 E+00$ & $1.00 E+00$ \\
\hline
\end{tabular}

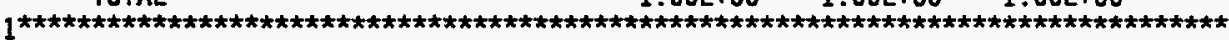

MOOAL STUDY

RISKIND-RW: RADIOACTIVE MATE File: riskind.in

03/23/95 16:36 PAGE 6

\begin{tabular}{llcc} 
NUCLIDE & INVENTORY(Ci) & FRAC-REL & Ci-REL \\
\hline CS 137 & $7.70 E+01$ & $1.00 E+00$ & $7.70 E+01$
\end{tabular}

1 1

MODAL STUDY $\quad 03 / 23 / 95 \quad 16: 36$ PAGE 7

RISKIND-RW: RADIOACTIVE MATE File: riskind.in

ONE YEAR COMMITTED ACUTE INHALATION DOSE CONVERSION FACTORS

\begin{tabular}{|c|c|c|c|c|c|}
\hline \multicolumn{6}{|c|}{ (RAD/SEC/PCI/M3) } \\
\hline NUCLIDE & R.MARROW & LUNG & GI-LLI & EFFECTIVE & GONADS \\
\hline $\operatorname{cs} 137$ & $9.80 E-12$ & $1.05 \mathrm{E}-11$ & $1.05 \mathrm{E}-11$ & $1.02 E-11$ & $9.45 E-12$ \\
\hline
\end{tabular}

50 YEAR CHRONIC INHALATION DOSE COMMITMENT FACTORS (REM/YR/PCI/M3)

NUCLIDE R.MARROW LUNG GI-LLI EFFECTIVE GONADS 


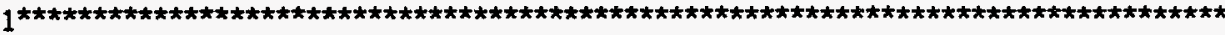

MODAL STUDY

RISKIND-RW: RADIOACTIVE MATE File: riskind.in

ACUTE CLOUD SHINE DOSE FACTORS (RAD/SEC/PCI/M3)

\begin{tabular}{|c|c|c|c|c|c|}
\hline NUCLIDE & R.MARROW & LUNG & GI-LLI & EFFECTIVE & GONADS \\
\hline $\operatorname{cs} 13$ & $9.14 E-14$ & $9.14 E-14$ & $9.14 E-14$ & $9.14 E-14$ & $9.14 E-14$ \\
\hline
\end{tabular}

\begin{tabular}{cccccc} 
NUCLIDE & R.MARROW & LUNG & GI-LLI & EFFECTIVE & GONADS \\
\hline CS137 & $1.83 E-15$ & $1.83 E-15$ & $1.83 E-15$ & $1.83 E-15$ & $1.83 E-15$
\end{tabular}

1 1

MODAL STUDY

RISKIND-RW: RADIOACTIVE MATE File: riskind.in

03/23/95 16:36 PAGE 9

CHRONIC CLOUD DOSE CONVERSION FACTORS (REM/YR/PCI/M3)

\begin{tabular}{llllll} 
NUCLIDE & R.MARROW & LUNG & GI-LLI & EFFECTIVE & GONADS \\
\hline CS137 & $2.89 E-06$ & $2.89 E-06$ & $2.89 E-06$ & $2.89 E-06$ & $2.89 E-06$
\end{tabular}

CHRONIC GROUND DOSE CONVERSION FACTORS (REM/YR/PCI/M2)

\begin{tabular}{llllll} 
NUCLIDE & R.MARROH & LUNG & GI-LLI & EFFECTIVE & GONADS \\
\hline CS137 & $5.76 E-08$ & $5.76 E-08$ & $5.76 E-08$ & $5.76 E-08$ & $5.76 E-08$ \\
\hline
\end{tabular}

\begin{tabular}{ccccrr} 
NUCLIDE & R.MARROW & LUNG & GI-LLI & EFFECTIVE & GONADS \\
\hline CS137 & $4.90 E-08$ & $4.72 E-08$ & $5.30 E-08$ & $5.02 E-08$ & $5.16 E-08$ \\
\hline
\end{tabular}

DISPERSION CALCULATION PARAMETERS (PUFIND):

PHYSICAL RELEASE HEIGHT = SOURCE EFFECTIVE SIZE =

ANAMETER HEIGHT =

AMBIENT TEMPERATURE =

DRY DEPOSITION VELOCITY = DEPOSITION VEL OVER WATER = DEPOSITION VEL OVER VEGETATION = STABILITY CLASS =

WIND SPEED =

SOURCE TEMPERATURE $=$

HEAT FLUX =

FINAL EFFECTIVE RELEASE HEIGHT =
$10.00(M)$

$0.14(\mathrm{M})$

$10.00(M)$

288.15 (K)

$0.0010(\mathrm{M} / \mathrm{S})$

$0.010(\mathrm{~m} / \mathrm{S})$

$0.010(\mathrm{M} / \mathrm{S})$

4

$4.00(\mathrm{M} / \mathrm{S})$

0.00 (K)

0.00 (CAL/SEC)

10.00 (M)

SUMMARY OF RECEPTOR CONCENTRATION AND DEPLETION FACTORS

\begin{tabular}{|c|c|c|c|c|c|c|}
\hline RCPTR & $\operatorname{DIST}(K M)$ & ANGLE & $\begin{array}{r}x O Q(G A S) \\
\left(S E C / M^{\star \star 3}\right)\end{array}$ & $\begin{array}{r}X D Q(P A R T) \\
\left(S E C / M^{\star \star} 3\right)\end{array}$ & $\begin{array}{l}\text { DEPLTION } \\
\text { FRACTION }\end{array}$ & $\begin{array}{c}\text { DEPOSITION } \\
\left(1 / M^{\star \star} 2\right)\end{array}$ \\
\hline 1 & 1.30E-01 & $0.00 E+00$ & $2.64 \mathrm{E}-04$ & $2.63 E-04$ & $2.55 \mathrm{E}-04$ & $2.63 E-07$ \\
\hline
\end{tabular}




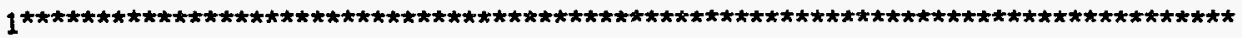

MODAL STUDY

RISKIND-RW: RADIOACTIVE MATE File: riskind.in

03/23/95 16:36 PAGE 10

SUMMARY OF RECEPTOR POTENTIAL DOSE CONSEQUENCES

FATALITY AND GENETIC RISKS

I LOCATION NAMES

\begin{tabular}{|c|c|c|c|c|}
\hline \multicolumn{2}{|c|}{ ACUTE } & \multicolumn{3}{|c|}{ LONG TERM } \\
\hline EDE & CHANCE & EDE & CHA & NCE \\
\hline REM & FATALITY & REM & FATALITY & GENETIC.E \\
\hline & $0.00 E+00$ & $1.22 E+\infty$ & $6.10 E-04$ & $1.59 \mathrm{E}-04$ \\
\hline
\end{tabular}

1 RECEPTOR XNAME (I) $2.29 E-01 \quad 0.00 E+00 \quad 1.22 E+00 \quad 6.10 E-04 \quad 1.59 E-04$

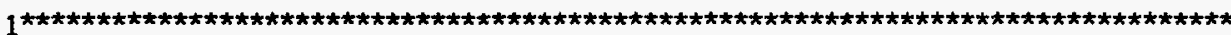

MODAL STUDY

RISKIND-RW: RADIOACTIVE MATE File: riskind.in

03/23/95 16:36 PAGE 11

TIME-INTEGRATED( 1 YEARS) DOSE CONSEQUENCE (REM)

RECEPTOR 1 : RECEPTOR XNAME (I)

DOWN WIND DIST $(K M)=1.30 E-01$

CROSS WIND DIST $(\mathrm{KM})=0.00 E+00$

\begin{tabular}{llllll} 
PATHWAY & R. MARROW & LUNG & GI-LLI & EFFECTIVE & GONADS \\
\hline PLUME & $2.21 E-01$ & $2.34 E-01$ & $2.36 E-01$ & $2.29 E-01$ & $2.33 E-01$ \\
EXT CASK & $2.42 E-07$ & $2.42 E-07$ & $2.42 E-07$ & $2.42 E-07$ & $2.42 E-07$ \\
RESUSPENSN. & $7.31 E-03$ & $7.76 E-03$ & $7.84 E-03$ & $7.60 E-03$ & $7.72 E-03$ \\
INGESTION & $4.57 E-01$ & $4.40 E-01$ & $4.94 E-01$ & $4.68 E-01$ & $4.81 E-01$ \\
EXT GROUND & $5.16 E-01$ & $5.16 E-01$ & $5.16 E-01$ & $5.16 E-01$ & $5.16 E-01$ \\
EXT CLOUD & $7.99 E-05$ & $7.99 E-05$ & $7.99 E-05$ & $7.99 E-05$ & $7.99 E-05$ \\
WATER & $0.00 E+00$ & $0.00 E+00$ & $0.00 E+00$ & $0.00 E+00$ & $0.00 E+00$ \\
\hline TOTAL & $1.20 E+00$ & $1.20 E+00$ & $1.25 E+00$ & $1.22 E+00$ & $1.24 E+00$
\end{tabular}

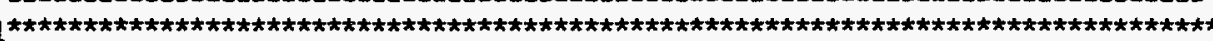

MODAL STUDY

RISKIND-RW: RADIOACTIVE MATE File: riskind.in

03/23/95 16:36 PAGE 12

SUMMARY OF PROBABILITY WEIGHTEO RISKS

RECEPTOR 1 : RECEPTOR XNAME (I)

DOWN WIND DIST $(\mathrm{KM})=1.30 \mathrm{E}-01$

CROSS WIND DIST $(\mathrm{KM})=0.00 E+00$

\begin{tabular}{ccccc} 
& \multicolumn{2}{c}{ INCIDENT FREE } & \multicolumn{2}{c}{ ACCIDENTS } \\
& $\begin{array}{c}\text { TRANSIT } \\
1 / \text { CASK }\end{array}$ & $\begin{array}{c}\text { STOP } \\
1 / \text { STOP }\end{array}$ & $\begin{array}{c}\text { AIR/GROUND } \\
1 / \text { EVENT }\end{array}$ & $\begin{array}{c}\text { WATER } \\
1 / \text { EVENT }\end{array}$ \\
\hline $\begin{array}{c}\text { EFFECTIVE DOSE } \\
\text { (REM) }\end{array}$ & $0.00 E+00$ & $0.00 E+00$ & $1.22 E+00$ & $0.00 E+00$ \\
$\begin{array}{c}\text { LATENT CANCER } \\
\text { MORTALITY } \\
\text { (CHANCE) }\end{array}$ & $0.00 E+00$ & $0.00 E+00$ & $6.10 E-04$ & $0.00 E+00$ \\
$\begin{array}{c}\text { GENETIC EFFECT } \\
\text { (CHANCE) }\end{array}$ & $0.00 E+00$ & $0.00 E+00$ & $1.59 E-04$ & $0.00 E+00$ \\
ACUTE MORTALITY \\
(CHANCE)
\end{tabular}




\section{D.3 VAL-B.OUT}

RADIATION DOSES INCLUDING ONLY BEEF INGESTION PATHWAY 


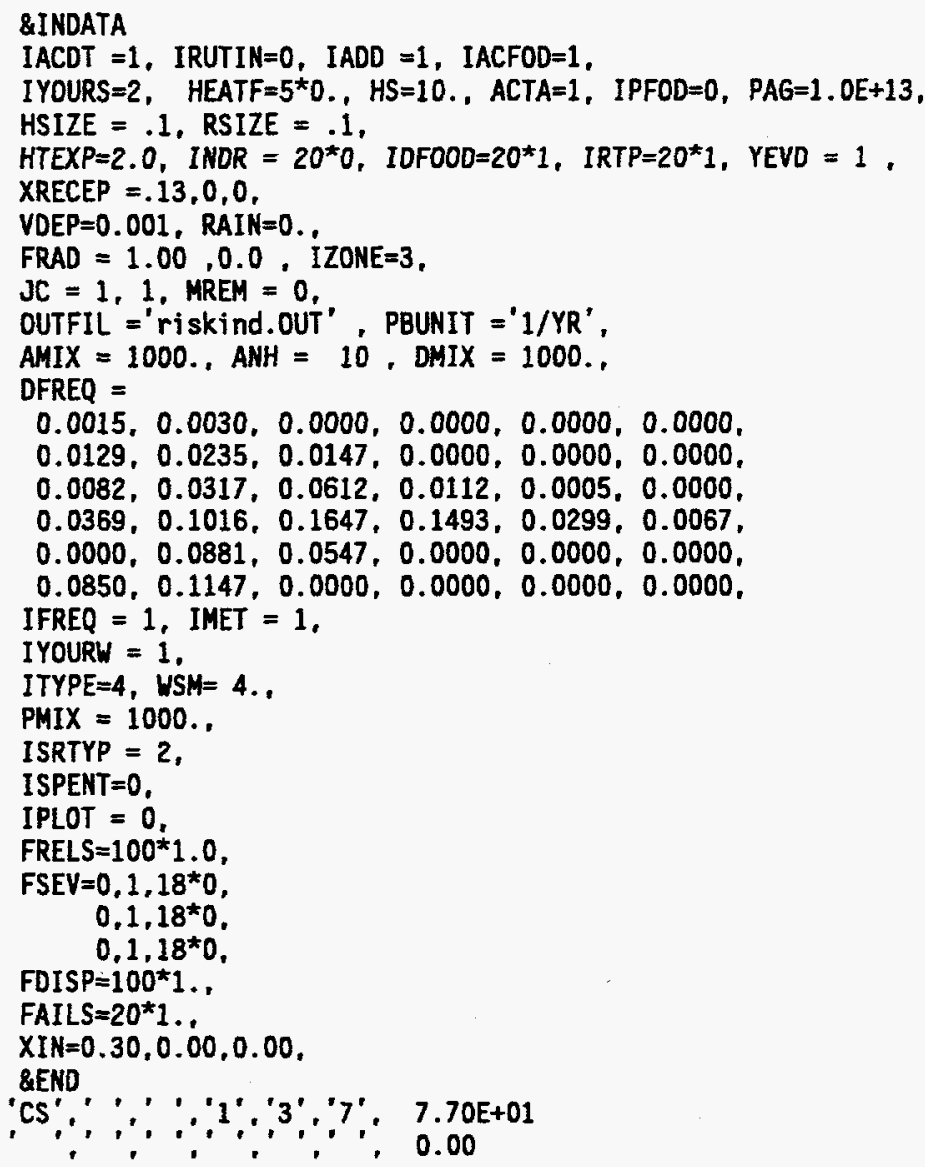




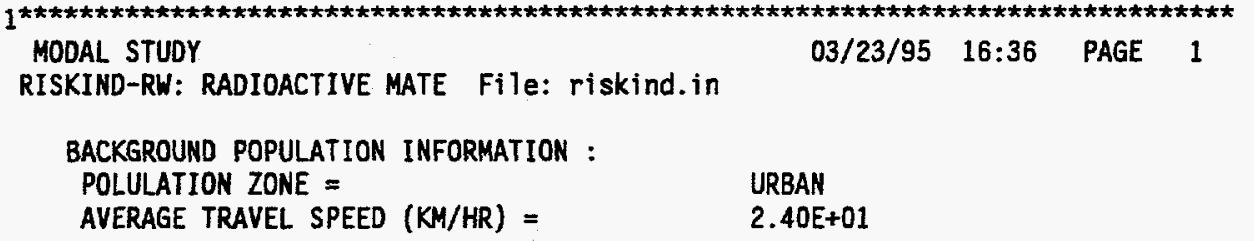

INDIVIDUAL RECEPTORS \& MISCELLANEOUS INPUT DATA

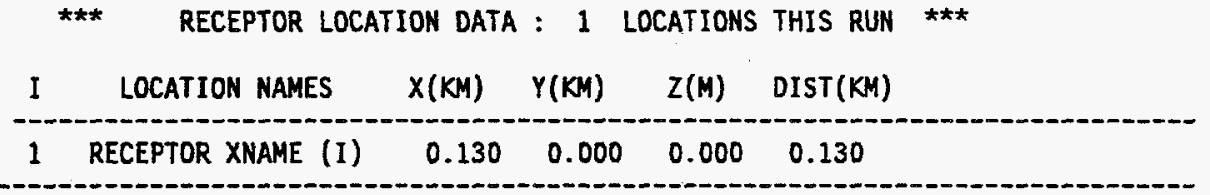

ACCIDENT DATA FROM STATE OF UT

$\begin{array}{ll}\text { ACCIDENT TRAFFIC FATALITY }(1 / \mathrm{KM})= & 0.00 \mathrm{E}+00 \\ \text { ACCIDENT PROBABILITY }(1 / \mathrm{KM})= & 1.00 \mathrm{E}+00\end{array}$

INDIVIDUAL DRINKING WATER INTAKE (L/DAY) $=1.00 E+00$

ALL INITIALLY CONTAMINATED FOOD STUFFS ARE DISCARDED

TRANSPORTATION MODE =

TRUCK

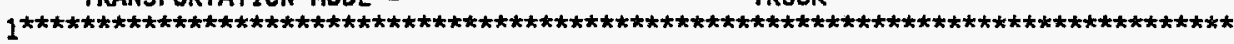

MODAL STUDY

RISKIND-RW: RADIOACTIVE MATE File: riskind.in

03/23/95 16:36 PAGE 2

FRACTION RELEASED ANO ALSO DISPERSED FOR EACH RESPONSE REGION

\begin{tabular}{|c|c|c|c|c|c|c|c|c|c|c|}
\hline \multirow{2}{*}{$\begin{array}{l}\text { NUCLIDE } \\
\text { TYPE }\end{array}$} & \multicolumn{10}{|c|}{ RESPONSE REGION } \\
\hline & 1 & 2 & 3 & 4 & 5 & 6 & 7 & 8 & 9 & 10 \\
\hline $\begin{array}{l}\text { PART. } \\
\text { RU } \\
\text { CS } \\
\text { IODINE } \\
\text { I.GAS }\end{array}$ & $\begin{array}{l}1 . E+00 \\
1 . E+00 \\
1 . E+00 \\
1 . E+00 \\
1 . E+00\end{array}$ & $\begin{array}{l}\text { 1. } E+00 \\
\text { 1. } E+00 \\
\text { 1. } E+00 \\
\text { 1. } E+00 \\
\text { 1. } E+00\end{array}$ & $\begin{array}{l}1 . E+00 \\
1 . E+00 \\
1 . E+00 \\
1 . E+00 \\
1 . E+00\end{array}$ & $\begin{array}{l}\text { 1. E+o0 } \\
1 . E+\infty 0 \\
1 . E+\infty 0 \\
1 . E+\infty 0 \\
1 . E+\infty 0\end{array}$ & $\begin{array}{l}1 . E+\infty 0 \\
1 . E+00 \\
1 . E+\infty 0 \\
1 . E+\infty 0 \\
1 . E+\infty 0\end{array}$ & $\begin{array}{l}\text { 1. } E+\infty 0 \\
\text { 1. } E+\infty 0 \\
1 . E+\infty 0 \\
1 . E+\infty 0 \\
1 . E+\infty\end{array}$ & $\begin{array}{l}\text { 1.E+D } \\
1 . E+\infty 0 \\
1 . E+\infty 0 \\
1 . E+\infty 0 \\
1 . E+\infty\end{array}$ & $\begin{array}{l}1 . E+00 \\
1 . E+00 \\
1 . E+00 \\
1 . E+00 \\
1 . E+00\end{array}$ & $\begin{array}{l}1 . E+00 \\
1 . E+00 \\
1 . E+00 \\
1 . E+00 \\
1 . E+00\end{array}$ & $\begin{array}{l}1 . E+00 \\
1 . E+00 \\
1 . E+00 \\
1 . E+00 \\
1 . E+00\end{array}$ \\
\hline PROB & $0 . E+00$ & 1. $E+00$ & $0 . E+00$ & $0 . E+00$ & $0 . E+00$ & $0 . E+00$ & $0 . E+00$ & $0 . E+00$ & $0 . E+00$ & $0 . E+00$ \\
\hline
\end{tabular}

FRACTION RELEASED ANO ALSO DISPERSED FOR EACH RESPONSE REGION

\begin{tabular}{|c|c|c|c|c|c|c|c|c|c|c|}
\hline \multirow{2}{*}{$\begin{array}{l}\text { NUCLIDE } \\
\text { TYPE }\end{array}$} & \multicolumn{10}{|c|}{ RESPONSE REGION } \\
\hline & 11 & 12 & 13 & 14 & 15 & 16 & 17 & 18 & 19 & 20 \\
\hline $\begin{array}{l}\text { PART. } \\
\text { RU } \\
\text { CS } \\
\text { IODINE } \\
\text { I.GAS }\end{array}$ & $\begin{array}{l}1 . E+00 \\
1 . E+00 \\
1 . E+00 \\
1 . E+00 \\
1 . E+00\end{array}$ & $\begin{array}{l}1 . E+\infty 0 \\
1 . E+00 \\
1 . E+00 \\
1 . E+00 \\
1 . E+00\end{array}$ & $\begin{array}{l}1 . E+00 \\
1 . E+00 \\
1 . E+00 \\
1 . E+00 \\
1 . E+00\end{array}$ & $\begin{array}{l}\text { 1. } E+\infty 0 \\
1 . E+\infty 0 \\
\text { 1. } E+\infty 0 \\
\text { 1. } E+\infty 0 \\
\text { 1. } E+\infty 0\end{array}$ & $\begin{array}{l}1 . E+00 \\
1 . E+00 \\
1 . E+00 \\
1 . E+00 \\
1 . E+00\end{array}$ & $\begin{array}{l}\text { 1. } E+00 \\
1 . E+00 \\
\text { 1. } E+00 \\
\text { 1. } E+00 \\
1 . E+00\end{array}$ & $\begin{array}{l}\text { 1. } E+\infty 0 \\
1 . E+00 \\
1 . E+00 \\
1 . E+\infty 0 \\
1 . E+00\end{array}$ & $\begin{array}{l}1 . E+00 \\
1 . E+00 \\
1 . E+00 \\
1 . E+00 \\
1 . E+00\end{array}$ & $\begin{array}{l}1 . E+00 \\
1 . E+00 \\
1 . E+00 \\
1 . E+00 \\
1 . E+00\end{array}$ & $\begin{array}{l}1 . E+\infty 0 \\
1 . E+\infty 0 \\
1 . E+\infty 0 \\
1 . E+\infty 0 \\
1 . E+\infty 0\end{array}$ \\
\hline PROB & $0 . E+00$ & $0 . E+00$ & $0 . E+\infty 0$ & $0 . E+00$ & $0 . E+00$ & $0 . E+00$ & $0 . E+00$ & $0 . E+00$ & $0 . E+00$ & $0 . E+00$ \\
\hline
\end{tabular}


1 1

MODAL STUDY

RISKIND-RW: RADIOACTIVE MATE File: riskind.in

03/23/95 16:36 PAGE 3

EXTERNAL DOSE RATE MULTIPLIER FOR EACH ACCIDENT RESPONSE REGION (MULTIPLIER OF TI)

\begin{tabular}{|c|c|c|c|c|c|c|c|c|c|c|}
\hline \multirow{2}{*}{ TYPE } & \multicolumn{10}{|c|}{ RESPONSE REGION } \\
\hline & 1 & 2 & 3 & 4 & 5 & 6 & 7 & 8 & 9 & 10 \\
\hline $\begin{array}{l}\text { GAMMA } \\
\text { NUTRN }\end{array}$ & $\begin{array}{l}1.00 \\
1.00\end{array}$ & $\begin{array}{l}2.00 \\
2.00\end{array}$ & $\begin{array}{l}2.00 \\
2.00\end{array}$ & $\begin{array}{l}2.00 \\
2.00\end{array}$ & $\begin{array}{l}2.00 \\
2.00\end{array}$ & $\begin{array}{l}2.00 \\
2.00\end{array}$ & $\begin{array}{l}2.00 \\
2.00\end{array}$ & $\begin{array}{l}2.00 \\
2.00\end{array}$ & $\begin{array}{l}2.00 \\
2.00\end{array}$ & $\begin{array}{l}2.00 \\
2.00\end{array}$ \\
\hline
\end{tabular}

EXTERNAL DOSE RATE MULTIPLIER FOR EACH ACCIDENT RESPONSE REGION (MULTIPLIER OF TI)

\begin{tabular}{lllllllllll} 
TYPE & \multicolumn{10}{c}{ RESPONSE REGION } \\
& 11 & 12 & 13 & 14 & 15 & 16 & 17 & 18 & 19 & 20 \\
\hline GANMA & 2.00 & 2.00 & 2.00 & 2.00 & 2.00 & 2.00 & 2.00 & 2.00 & 2.00 & 2.00 \\
NUTRN & 2.00 & 2.00 & 2.00 & 2.00 & 2.00 & 2.00 & 2.00 & 2.00 & 2.00 & 2.00 \\
\hline
\end{tabular}

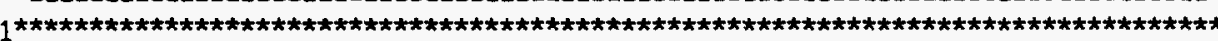

MODAL STUDY

RISKIND-RW: RADIOACTIVE MATE File: riskind.in

03/23/95 16:36 PAGE 4

$\begin{array}{lr}\text { CASK SIZE : RADIUS }(M)= & 1.00 E-01 \\ \text { LENGTH }(M)= & 1.00 E-01 \\ \text { FRACTION GANMA SOURCE }= & 1.00 \\ \text { FRACTION NEUTRON SOURCE } & 0.00 \\ \text { TRANSPORTATION INDEX }= & 10.00\end{array}$

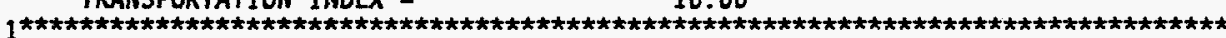

MODAL STUDY $\quad 03 / 23 / 95 \quad 16: 36$ PAGE 5

RISKIND-RW: RADIOACTIVE MATE File: riskind.in

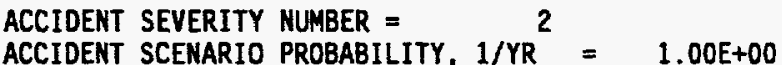

\begin{tabular}{|c|c|c|c|c|c|}
\hline NUCLIDE & $\operatorname{DCAY}(1 / Y R)$ & CURIES & \multicolumn{3}{|c|}{ RELATIVE HAZARD } \\
\hline & & & PLUME & INGESTION & G.SHINE \\
\hline CS 137 & $2.30 \mathrm{E}-02$ & $7.70 E+01$ & $1.00 E+00$ & $1.00 E+00$ & $1.00 E+00$ \\
\hline
\end{tabular}

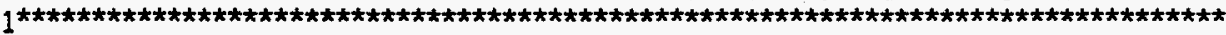

MODAL STUDY

RISKIND-RW: RADIOACTIVE MATE File: riskind.in

03/23/95 16:36 PAGE 6

\begin{tabular}{lccc} 
NUCLIDE & INVENTORY(Ci) & FRAC-REL & Ci-REL \\
\hline CS 137 & $7.70 E+01$ & $1.00 E+00$ & $7.70 E+01$
\end{tabular}

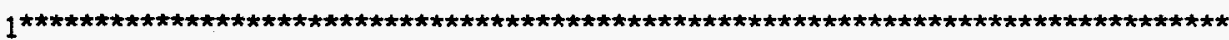

MODAL STUDY

RISKIND-RW: RADIOACTIVE MATE File: riskind.in

03/23/95 16:36 PAGE 7

ONE YEAR COMMITTED ACUTE INHALATION DOSE CONVERSION FACTORS (RAD/SEC/PCI/M3)

\begin{tabular}{cccccr} 
NUCLIDE & R.MARROW & LUNG & GI-LLI & EFFECTIVE & GONADS \\
\hline CS137 & $9.80 E-12$ & $1.05 E-11$ & $1.05 E-11$ & $1.02 E-11$ & $9.45 E-12$
\end{tabular}

50 YEAR CHRONIC INHALATION DOSE COMMITMENT FACTORS (REM/YR/PCI/M3)

NUCLIDE R.MARROW LUNG GI-LLI EFFECTIVE GONADS 
1 1

MODAL STUDY

RISKIND-RW: RADIOACTIVE MATE File: riskind.in

ACUTE CLOUD SHINE DOSE FACTORS (RAD/SEC/PCI/M3)

\begin{tabular}{|c|c|c|c|c|c|}
\hline NUCLIDE & R. MARROW & LUNG & GI-LLI & EFFECTIVE & GONADS \\
\hline CS137 & $9.14 \mathrm{E}-14$ & $9.14 \mathrm{E}-14$ & $9.14 E-14$ & $9.14 E-14$ & $9.14 \mathrm{E}-14$ \\
\hline
\end{tabular}

ACUTE GROUND SHINE DOSE FACTORS (RAD/SEC/PCI/M2)

\begin{tabular}{llllll} 
NUCLIDE & R.MARROW & LUNG & GI-LLI & EFFECTIVE & GONADS \\
\hline CS137 & $1.83 E-15$ & $1.83 E-15$ & $1.83 E-15$ & $1.83 E-15$ & $1.83 E-15$
\end{tabular}

1

MODAL STUDY

RISKIND-RW: RADIOACTIVE MATE File: riskind.in

03/23/95 16:36 PAGE 9

CHRONIC CLOUD DOSE CONVERSION FACTORS (REM/YR/PCI/M3)

\begin{tabular}{cccccr} 
NUCLIDE & R.MARROW & LUNG & GI-LLI & EFFECTIVE & GONADS \\
\hline CS137 & $2.89 E-06$ & $2.89 E-06$ & $2.89 E-06$ & $2.89 E-06$ & $2.89 E-06$
\end{tabular}

CHRONIC GROUND DOSE CONVERSION FACTORS (REM/YR/PCI/M2)

\begin{tabular}{|c|c|c|c|c|c|}
\hline NUCLIDE & R.MARROW & LUNG & GI-LLI & EFFECTIVE & GONADS \\
\hline CS137 & $5.76 E-08$ & $5.76 E-08$ & $5.76 E-08$ & $5.76 \mathrm{E}-08$ & $5.76 E-08$ \\
\hline
\end{tabular}

\begin{tabular}{|c|c|c|c|c|c|}
\hline NUCLIDE & $\begin{array}{c}\text { INGESTION DOSE } \\
\text { R.MARROW }\end{array}$ & $\begin{array}{l}\text { ONVERSION } \\
\text { LUNG }\end{array}$ & $\begin{array}{l}\text { ACTORS (RE } \\
\text { GI-LLI }\end{array}$ & $\begin{array}{l}\text { PCI) } \\
\text { EFFECTIVE }\end{array}$ & GONADS \\
\hline CS137 & $4.90 \mathrm{E}-08$ & $4.72 E-08$ & $5.30 \mathrm{E}-08$ & $5.02 E-08$ & $5.16 E-08$ \\
\hline
\end{tabular}

DISPERSION CALCULATION PARAMETERS (PUFIND):

PHYSICAL RELEASE HEIGHT = SOURCE EFFECTIVE SIZE =

ANANETER HEIGHT =

AMBIENT TEMPERATURE =

ORY DEPOSITION VELOCITY =

DEPOSITION VEL OVER HATER =

DEPOSITION VEL OVER VEGETATION =

STABILITY CLASS =

WIND SPEED =

SOURCE TEMPERATURE =

HEAT FLUX $=$

FINAL EFFECTIVE RELEASE HEIGHT =

$10.00(\mathrm{M})$
$0.14(\mathrm{M})$
$10.00(\mathrm{M})$
$288.15(\mathrm{~K})$
$0.0010(\mathrm{M} / \mathrm{S})$
$0.010(\mathrm{M} / \mathrm{S})$
$0.010(\mathrm{M} / \mathrm{S})$
4
$4.00(\mathrm{M} / \mathrm{S})$
$0.00(\mathrm{~K})$
$0.00(\mathrm{CAL} / \mathrm{SEC})$
$10.00(\mathrm{M})$

$10.00(M)$

SUMMARY OF RECEPTOR CONCENTRATION AND DEPLETION FACTORS

\begin{tabular}{lcccccc} 
RCPTR & DIST(KM) & ANGLE & $\begin{array}{c}\text { XOQ(GAS) } \\
\left(\mathrm{SEC} / M^{\star *} 3\right)\end{array}$ & $\begin{array}{c}\text { XOQ(PART) } \\
\left(\mathrm{SEC} / M^{\star *} 3\right)\end{array}$ & $\begin{array}{c}\text { DEPLTION } \\
\text { FRACTION }\end{array}$ & $\begin{array}{c}\text { DEPOSITION } \\
\left(1 / M^{\star *} 2\right)\end{array}$ \\
\hline 1 & $1.30 E-01$ & $0.00 E+00$ & $2.64 E-04$ & $2.63 E-04$ & $2.55 E-04$ & $2.63 E-07$ \\
\hline
\end{tabular}


SUMMARY OF RECEPTOR POTENTIAL DOSE CONSEQUENCES

FATALITY AND GENETIC RISKS

I LOCATION NAMES

ACUTE LONG TERM

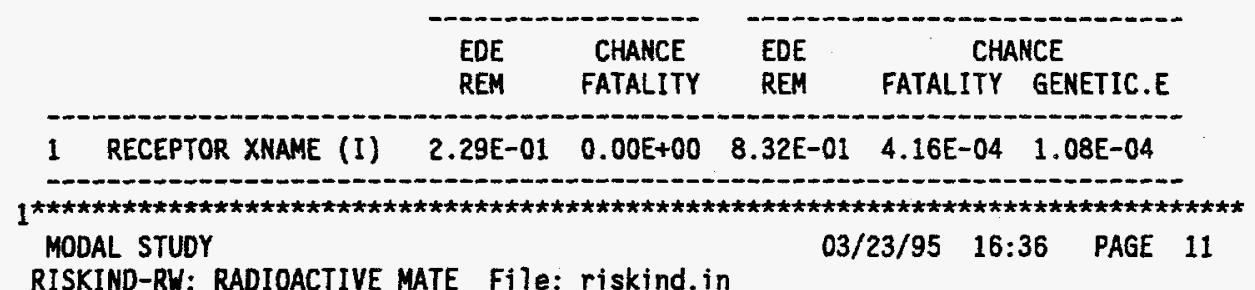

TIME-INTEGRATEO( 1 YEARS) DOSE CONSEQUENCE (REM)

RECEPTOR 1 : RECEPTOR XNAME (I)

DOWN WIND DIST $(\mathrm{KM})=1.30 E-01$

CROSS WIND DIST $(\mathrm{KM})=0.00 E+00$

\begin{tabular}{lccccc} 
PATHWAY & R.MARROW & LUNG & GI-LLI & EFFECTIVE & GONADS \\
\hline PLUME & $2.21 E-01$ & $2.34 E-01$ & $2.36 E-01$ & $2.29 E-01$ & $2.33 E-01$ \\
EXT CASK & $2.42 E-07$ & $2.42 E-07$ & $2.42 E-07$ & $2.42 E-07$ & $2.42 E-07$ \\
RESUSPENSN. & $7.31 E-03$ & $7.76 E-03$ & $7.84 E-03$ & $7.60 E-03$ & $7.72 E-03$ \\
INGESTION & $7.69 E-02$ & $7.40 E-02$ & $8.32 E-02$ & $7.87 E-02$ & $8.09 E-02$ \\
EXT GROUND & $5.16 E-01$ & $5.16 E-01$ & $5.16 E-01$ & $5.16 E-01$ & $5.16 E-01$ \\
EXT CLOUD & $7.99 E-05$ & $7.99 E-05$ & $7.99 E-05$ & $7.99 E-05$ & $7.99 E-05$ \\
WATER & $0.00 E+00$ & $0.00 E+00$ & $0.00 E+00$ & $0.00 E+00$ & $0.00 E+00$ \\
\hline TOTAL & $8.21 E-01$ & $8.32 E-01$ & $8.44 E-01$ & $8.32 E-01$ & $8.38 E-01$
\end{tabular}

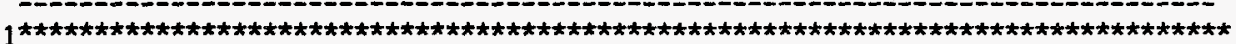

MODAL STUDY

RISKIND-RW: RADIOACTIVE MATE File: riskind.in

03/23/95 16:36 PAGE 12

\begin{tabular}{|c|c|c|c|c|}
\hline & \multicolumn{4}{|c|}{$\begin{array}{l}\text { SUMMARY OF PROBABILITY WEIGHTED RISKS } \\
\text { RECEPTOR } 1 \text { : RECEPTOR XNAME (I) } \\
\text { DOWN WIND DIST (KM) = } 1.30 E-01 \\
\text { CROSS WIND DIST }(\mathrm{KM})=0.00 E+00\end{array}$} \\
\hline & $\begin{array}{l}\text { INCID } \\
\text { TRANSIT } \\
\text { 1/CASK }\end{array}$ & $\begin{array}{l}\text { FREE } \\
\text { STOP } \\
\text { I/STOP }\end{array}$ & $\begin{array}{l}\text { AC } \\
\text { AIR/GROUND } \\
\text { 1/EVENT }\end{array}$ & $\begin{array}{l}\text { ENTS } \\
\text { WATER } \\
1 / \text { EVENT }\end{array}$ \\
\hline $\begin{array}{l}\text { EFFECTIVE DOSE } \\
\text { (REM) } \\
\text { LATENT CANCER }\end{array}$ & $0.00 E+00$ & $0.00 E+00$ & $8.32 E-01$ & $0.00 E+00$ \\
\hline $\begin{array}{l}\text { MORTALITY } \\
\text { (CHANCE) }\end{array}$ & $0.00 E+00$ & $0.00 E+00$ & $4.16 E-04$ & $0.00 \mathrm{E}+00$ \\
\hline $\begin{array}{l}\text { GENETIC EFFECT } \\
\text { (CHANCE) } \\
\text { ACUTE MORTALITY } \\
\text { (CHANCE) }\end{array}$ & $0.00 E+00$ & $0.00 E+00$ & $\begin{array}{l}1.08 E-04 \\
0.00 E+00\end{array}$ & $0.00 E+00$ \\
\hline
\end{tabular}




\section{D.4 VAL-M.OUT}

RADIATION DOSES INCLUDING ONLY MILK INGESTION PATHWAY 


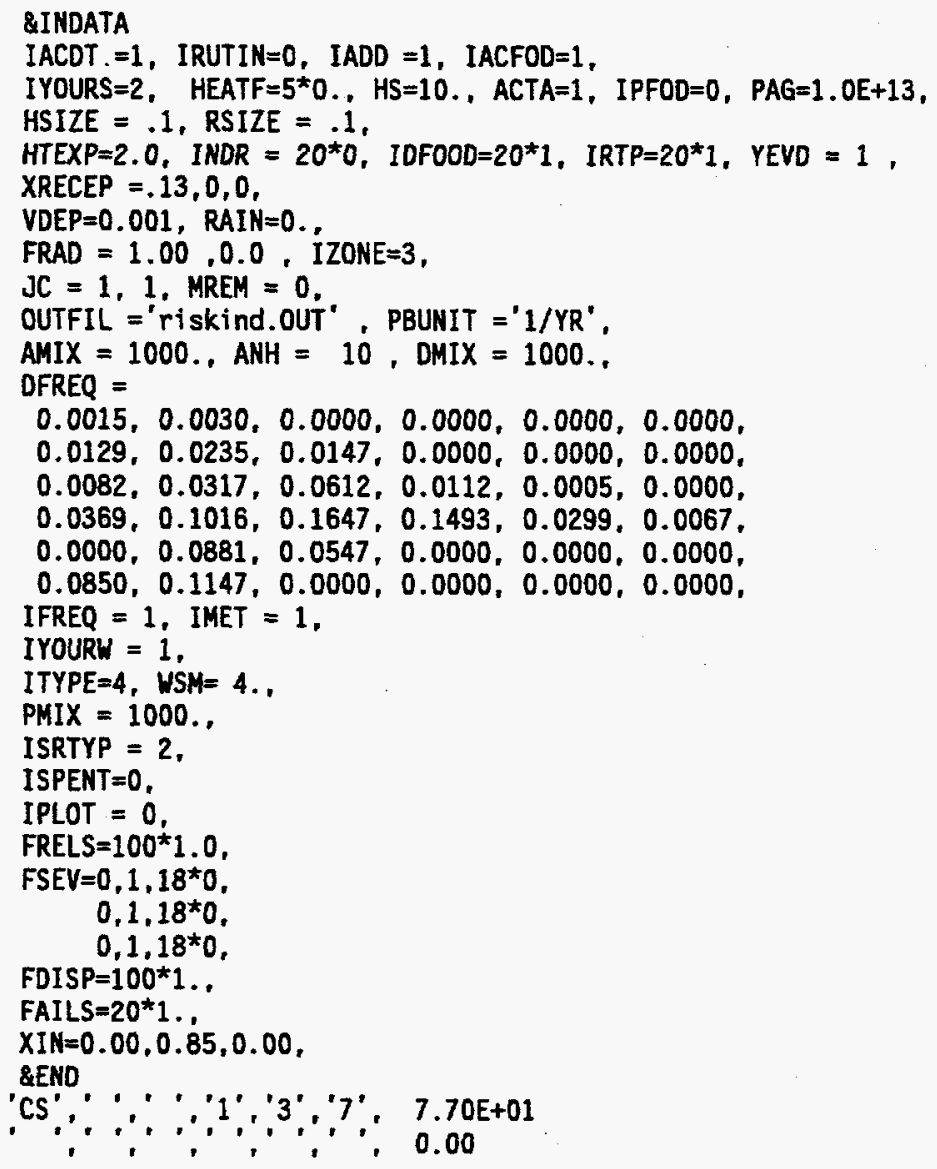




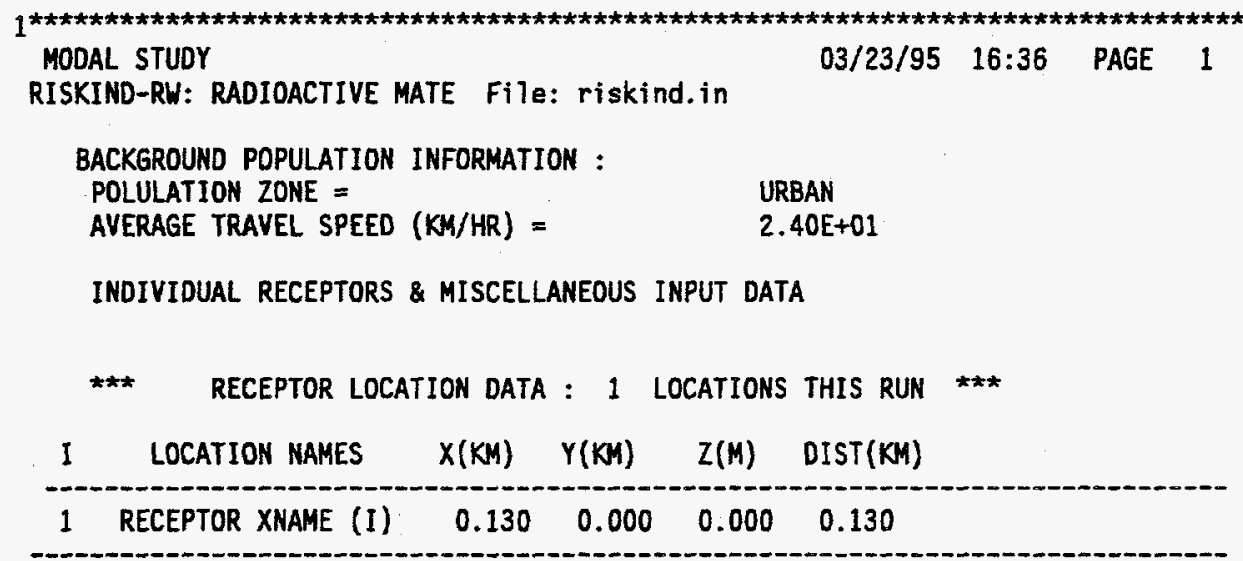

ACCIDENT DATA FROM STATE OF UT

ACCIDENT TRAFFIC FATALITY $(1 / \mathrm{KM})=0.00 E+00$

ACCIDENT PROBABILITY $(1 / \mathrm{KM})=\quad 1.00 \mathrm{E}+00$

INDIVIDUAL DRINKING WATER INTAKE (L/DAY) $=1.00 E+\infty 0$

ALL INITIALLY CONTAMINATED FOOD STUFFS ARE DISCARDED

TRANSPORTATION MODE =

TRUCK

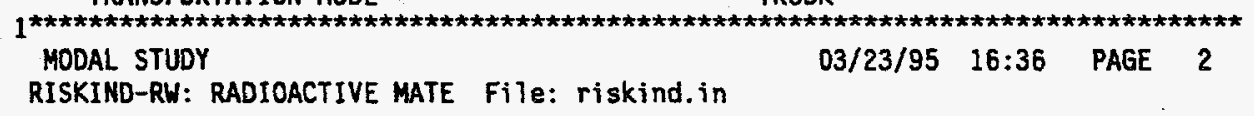

FRACTION RELEASED AND ALSO DISPERSED FOR EACH RESPONSE REGION

\begin{tabular}{|c|c|c|c|c|c|c|c|c|c|c|}
\hline \multirow{2}{*}{$\begin{array}{l}\text { NUCLIDE } \\
\text { TYPE }\end{array}$} & \multicolumn{10}{|c|}{ RESPONSE REGION } \\
\hline & 1 & 2 & 3 & 4 & 5 & 6 & 7 & 8 & 9 & 10 \\
\hline $\begin{array}{l}\text { PART. } \\
\text { RU } \\
\text { CS } \\
\text { IODINE } \\
\text { I.GAS }\end{array}$ & $\begin{array}{l}\text { 1. } E+\infty 0 \\
1 . E+00 \\
1 . E+\infty D \\
1 . E+00 \\
1 . E+\infty 0\end{array}$ & $\begin{array}{l}\text { 1. } E+\infty 0 \\
1 . E+00 \\
1 . E+00 \\
1 . E+00 \\
1 . E+\infty 0\end{array}$ & $\begin{array}{l}1 . E+00 \\
1 . E+00 \\
1 . E+00 \\
1 . E+00 \\
1 . E+00\end{array}$ & $\begin{array}{l}1 . E+00 \\
1 . E+00 \\
1 . E+00 \\
1 . E+00 \\
1 . E+00\end{array}$ & $\begin{array}{l}\text { 1. } E+00 \\
\text { 1. } E+00 \\
\text { 1. } E+00 \\
\text { 1. } E+00 \\
\text { 1. } E+00\end{array}$ & $\begin{array}{l}1 . E+00 \\
1 . E+00 \\
1 . E+00 \\
1 . E+00 \\
1 . E+00\end{array}$ & $\begin{array}{l}1 . E+00 \\
1 . E+00 \\
1 . E+00 \\
1 . E+00 \\
1 . E+00\end{array}$ & $\begin{array}{l}1 . E+\infty 0 \\
1 . E+\infty 0 \\
1 . E+\infty 0 \\
1 . E+00 \\
1 . E+\infty 0\end{array}$ & $\begin{array}{l}1 . E+00 \\
1 . E+00 \\
1 . E+00 \\
1 . E+00 \\
1 . E+00\end{array}$ & $\begin{array}{l}1 . E+00 \\
1 . E+00 \\
1 . E+00 \\
1 . E+00 \\
1 . E+00\end{array}$ \\
\hline PROB & $0 . E+00$ & 1. $E+00$ & $0 . E+00$ & $0 . E+00$ & $0 . E+00$ & $0 . E+00$ & $0 . E+\infty 0$ & $0 . E+00$ & $0 . E+00$ & $0 . E+00$ \\
\hline
\end{tabular}

FRACTION RELEASED AND ALSO DISPERSED FOR EACH RESPONSE REGION

\begin{tabular}{|c|c|c|c|c|c|c|c|c|c|c|}
\hline \multirow{2}{*}{$\begin{array}{l}\text { NUCLIDE } \\
\text { TYPE }\end{array}$} & \multicolumn{10}{|c|}{ RESPONSE REGION } \\
\hline & 11 & 12 & 13 & 14 & 15 & 16 & 17 & 18 & 19 & 20 \\
\hline $\begin{array}{l}\text { PART. } \\
\text { RU } \\
\text { CS } \\
\text { IODINE } \\
\text { I. GAS }\end{array}$ & $\begin{array}{l}\text { 1. } E+00 \\
1 . E+00 \\
1 . E+\infty 0 \\
1 . E+\infty 0 \\
1 . E+\infty 0\end{array}$ & $\begin{array}{l}\text { 1. } E+00 \\
1 . E+\infty 0 \\
1 . E+00 \\
1 . E+\infty 0 \\
1 . E+\infty 0\end{array}$ & $\begin{array}{l}\text { 1. } E+\infty 0 \\
\text { 1. } E+\infty 0 \\
\text { 1. } E+\infty 0 \\
\text { 1. } E+\infty 0 \\
\text { 1. } E+\infty O\end{array}$ & $\begin{array}{l}1 . E+\infty 0 \\
1 . E+00 \\
1 . E+00 \\
1 . E+00 \\
1 . E+00\end{array}$ & $\begin{array}{l}\text { 1. } E+00 \\
1 . E+00 \\
1 . E+00 \\
1 . E+00 \\
1 . E+00\end{array}$ & $\begin{array}{l}\text { 1. } E+\infty 0 \\
1 . E+\infty 0 \\
1 . E+\infty 0 \\
\text { 1. } E+\infty 0 \\
1 . E+\infty 0\end{array}$ & $\begin{array}{l}1 . E+00 \\
1 . E+00 \\
1 . E+00 \\
1 . E+00 \\
1 . E+00\end{array}$ & $\begin{array}{l}1 . E+\infty 0 \\
1 . E+00 \\
1 . E+00 \\
1 . E+00 \\
1 . E+00\end{array}$ & $\begin{array}{l}1 \cdot E+00 \\
1 . E+00 \\
1 . E+00 \\
1 \cdot E+00 \\
1 . E+00\end{array}$ & $\begin{array}{l}1 . E+00 \\
1 . E+00 \\
1 . E+00 \\
1 . E+00 \\
1 . E+00\end{array}$ \\
\hline PROB & $0 . E+00$ & D.E+OD & $0 . E+00$ & $0 . E+00$ & $0 . E+00$ & $0 . E+O 0$ & $0 . E+00$ & D. E+00 & $0 . E+00$ & $0 . E+00$ \\
\hline
\end{tabular}




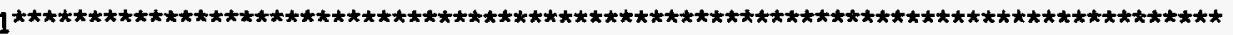

MODAL STUDY

RISKIND-RW: RADIOACTIVE MATE File: riskind.in

03/23/95 16:36 PAGE 3

EXTERNAL DOSE RATE MULTIPLIER FOR EACH ACCIDENT RESPONSE REGION (MULTIPLIER OF TI)

\begin{tabular}{|c|c|c|c|c|c|c|c|c|c|c|}
\hline \multirow{2}{*}{ TYPE } & \multicolumn{10}{|c|}{$\begin{array}{l}\text { RESPONSE REGION } \\
\text { REN }\end{array}$} \\
\hline & 1 & 2 & 3 & 4 & 5 & 6 & 7 & 8 & 9 & 10 \\
\hline $\begin{array}{l}\text { GAMMA } \\
\text { NUTRN }\end{array}$ & $\begin{array}{l}1.00 \\
1.00\end{array}$ & $\begin{array}{l}2.00 \\
2.00\end{array}$ & $\begin{array}{l}2.00 \\
2.00\end{array}$ & $\begin{array}{l}2.00 \\
2.00\end{array}$ & $\begin{array}{l}2.00 \\
2.00\end{array}$ & $\begin{array}{l}2.00 \\
2.00\end{array}$ & $\begin{array}{l}2.00 \\
2.00\end{array}$ & $\begin{array}{l}2.00 \\
2.00\end{array}$ & $\begin{array}{l}2.00 \\
2.00\end{array}$ & $\begin{array}{l}2.00 \\
2.00\end{array}$ \\
\hline
\end{tabular}

EXTERNAL DOSE RATE MULTIPLIER FOR EACH ACCIDENT RESPONSE REGION (MULTIIPLIER OF TI)

\begin{tabular}{|c|c|c|c|c|c|c|c|c|c|c|}
\hline \multirow[t]{2}{*}{ TYPE } & \multicolumn{10}{|c|}{ RESPONSE REGION } \\
\hline & 11 & 12 & 13 & 14 & 15 & 16 & 17 & 18 & 19 & 20 \\
\hline $\begin{array}{l}\text { GAMMA } \\
\text { NUTRN }\end{array}$ & $\begin{array}{l}2.00 \\
2.00\end{array}$ & $\begin{array}{l}2.00 \\
2.00\end{array}$ & $\begin{array}{l}2.00 \\
2.00\end{array}$ & $\begin{array}{l}2.00 \\
2.00\end{array}$ & $\begin{array}{l}2.00 \\
2.00\end{array}$ & $\begin{array}{l}2.00 \\
2.00\end{array}$ & $\begin{array}{l}2.00 \\
2.00\end{array}$ & $\begin{array}{l}2.00 \\
2.00\end{array}$ & $\begin{array}{l}2.00 \\
2.00\end{array}$ & $\begin{array}{l}2.00 \\
2.00\end{array}$ \\
\hline
\end{tabular}

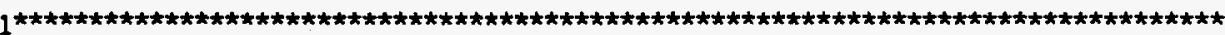

MODAL STUDY

RISKIND-RW: RADIOACTIVE MATE File: riskind.in

03/23/95 16:36 PAGE 4

$\begin{array}{rr}\text { CASK SIZE : RADIUS }(M)= & 1.00 E-01 \\ \text { LENGTH }(M)= & 1.00 E-01 \\ \text { FRACTION GANMA SOURCE }= & 1.00 \\ \text { FRACTION NEUTRON SOURCE }= & 0.00 \\ \text { TRANSPORTATION INDEX }= & 10.00\end{array}$

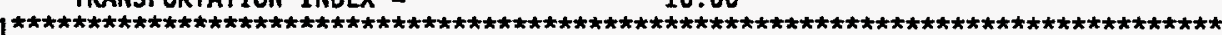

MODAL STUDY $\quad 03 / 23 / 95 \quad 16: 36$ PAGE 5

RISKIND-RW: RADIOACTIVE MATE File: riskind.in

ACCIDENT SEVERITY NUMBER $=$
ACCIDENT SCENARIO PROBABILITY, $1 / \mathrm{YR}^{2}=1.00 E+00$

\begin{tabular}{|c|c|c|c|c|c|c|c|}
\hline NUCLIDE & $\operatorname{DCAY}(1 / Y R)$ & CURIES & & ELATIVE HAZ & ZARO & & \\
\hline & & & PLUME & INGESTION & & INE & \\
\hline CS 137 & 2.30E-02 & $7.70 E+01$ & 1. $.00 E+00$ & $1.00 E+00$ & & $E+00$ & \\
\hline$\underset{\star \star \star \star \star \star \star * \star \star \star *}{\text { TOTAL }}$ & & & 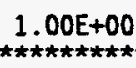 & 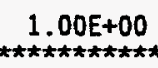 & 1. & $\begin{array}{l}E+00 \\
\star \star \star \star \star \star\end{array}$ & \\
\hline $\begin{array}{l}\text { MODAL STUDY } \\
\text { RISKIND-RW: }\end{array}$ & OACTIVE MA & File. $r$ & & $03 / 23 / 95$ & $16: 36$ & PAGE & 6 \\
\hline
\end{tabular}

RISKIND-RW: RADIOACTIVE MATE File: riskind.in

\begin{tabular}{lccc} 
NUCLIDE & INVENTORY (Ci) & FRAC-REL & Ci-REL \\
\hline CS 137 & $7.70 E+01$ & $1.00 E+00$ & $7.70 E+01$
\end{tabular}

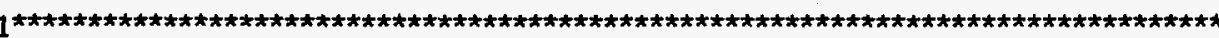

MODAL STUDY $103 / 23 / 95 \quad 16: 36 \quad$ PAGE 7

RISKIND-RW: RADIOACTIVE MATE File: riskind.in

ONE YEAR COMMITTED ACUTE INHALATION DOSE CONVERSION FACTORS

\begin{tabular}{cccccc} 
NUCLIDE & \multicolumn{5}{c}{ (RAD/SEC/PCI/M3) } \\
\hline CS137 & $9.80 E-12$ & $1.05 E-11$ & $1.05 E-11$ & $1.02 E-11$ & $9.45 E-12$
\end{tabular}

50 YEAR CHRONIC INHALATION DOSE COMMITMENT FACTORS (REM/YR/PCI/M3)

NUCLIDE R.MARROW LUNG GI-LLI EFFECTIVE GONADS 
MODAL STUDY

03/23/95 16:36 PAGE 8

RISKIND-RW: RADIOACTIVE MATE File: riskind.in

ACUTE CLOUD SHINE DOSE FACTORS (RAD/SEC/PCI/M3)

\begin{tabular}{cccccc} 
NUCLIDE & R.MARROW & LUNG & GI-LLI & EFFECTIVE & GONADS \\
\hline CS137 & $9.14 E-14$ & $9.14 E-14$ & $9.14 E-14$ & $9.14 E-14$ & $9.14 E-14$
\end{tabular}

ACUTE GROUND SHINE DOSE FACTORS (RAD/SEC/PCI/M2)

\begin{tabular}{cccccc} 
NUCLIDE & R.MARROW & LUNG & GI-LLI & EFFECTIVE & GONADS \\
\hline CS137 & $1.83 E-15$ & $1.83 \mathrm{E}-15$ & $1.83 \mathrm{E}-15$ & $1.83 \mathrm{E}-15$ & $1.83 \mathrm{E}-15$
\end{tabular}

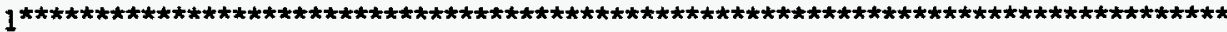

MODAL STUDY

RISKIND-RW: RADIOACTIVE MATE File: riskind.in

$$
03 / 23 / 95 \text { 16:36 PAGE } 9
$$

CHRONIC CLOUD DOSE CONVERSION FACTORS (REM/YR/PCI/M3)

\begin{tabular}{llllll}
\multicolumn{1}{c}{ NUCLIDE } & R.MARROW & LUNG & GI-LLI & EFFECTIVE & GONADS \\
\hline CS137 & $2.89 E-06$ & $2.89 E-06$ & $2.89 E-06$ & $2.89 E-06$ & $2.89 E-06$
\end{tabular}

CHRONIC GROUND DOSE CONVERSION FACTORS (REM/YR/PCI/M2)

\begin{tabular}{llllll} 
NUCLIDE & R.MARROW & LUNG & GI-LLI & EFFECTIVE & GONADS \\
\hline CS137 & $5.76 E-08$ & $5.76 E-08$ & $5.76 E-08$ & $5.76 E-08$ & $5.76 E-08$
\end{tabular}

\begin{tabular}{cccccc} 
NUCLIDE & $\begin{array}{c}\text { INGESTION DOSE CONVERSION FACTORS (REM/PCI) } \\
\text { R.MARROW }\end{array}$ LUNG & GI-LLI & EFFECTIVE & GONADS \\
\hline CS137 & $4.90 E-08$ & $4.72 E-08$ & $5.30 E-08$ & $5.02 E-08$ & $5.16 E-08$
\end{tabular}

OISPERSION CALCULATION PARAMETERS (PUFIND):

PHYSICAL RELEASE HEIGHT = SOURCE EFFECTIVE SIZE =

ANAMETER HEIGHT =

AMBIENT TEMPERATURE =

DRY DEPOSITION VELOCITY =

DEPOSITION VEL OVER WATER =

DEPOSITION VEL OVER VEGETATION =

STABILITY CLASS =

WIND SPEED =

SOURCE TEMPERATURE :

HEAT FLUX =

FINAL EFFECTIVE RELEASE HEIGHT =

$10.00(\mathrm{M})$
$0.14(\mathrm{M})$
$10.00(\mathrm{M})$
$288.15(\mathrm{~K})$
$0.0010(\mathrm{M} / \mathrm{S})$
$0.010(\mathrm{M} / \mathrm{S})$
$0.010(\mathrm{M} / \mathrm{S})$
4
$4.00(\mathrm{M} / \mathrm{S})$
$0.00(\mathrm{~K})$
$0.00(\mathrm{CAL} / \mathrm{SEC})$
$10.00(\mathrm{M})$

$10.00(\mathrm{M})$

SUMMARY OF RECEPTOR CONCENTRATION AND DEPLETION FACTORS

\begin{tabular}{|c|c|c|c|c|c|c|}
\hline RCPTR & $\operatorname{DIST}(K N)$ & ANGLE & $\begin{array}{r}X O Q(\text { GAS }) \\
\left(\mathrm{SEC} / \mathrm{M}^{\star *} 3\right)\end{array}$ & $\begin{array}{r}X 0 Q(\text { PART) } \\
\left(\mathrm{SEC} / M^{\star \star} 3\right)\end{array}$ & $\begin{array}{l}\text { DEPLTION } \\
\text { FRACTION }\end{array}$ & $\begin{array}{c}\text { DEPOSITION } \\
\left(1 / M^{\star \star *} 2\right)\end{array}$ \\
\hline 1 & $1.30 E-01$ & $0.00 E+00$ & $2.64 E-04$ & $2.63 E-04$ & $2.55 E-04$ & $2.63 E-07$ \\
\hline
\end{tabular}


RISKIND-RW: RADIOACTIVE MATE File: riskind.in

SUMMARY OF RECEPTOR POTENTIAL DOSE CONSEQUENCES

FATALITY AND GENETIC RISKS

I LOCATION NAMES

\begin{tabular}{|c|c|c|c|c|}
\hline \multicolumn{2}{|c|}{ ACUTE } & \multicolumn{3}{|c|}{ LONG TERM } \\
\hline $\begin{array}{l}\text { EDE } \\
\text { REN }\end{array}$ & $\begin{array}{l}\text { CHANCE } \\
\text { FATALITY }\end{array}$ & $\begin{array}{l}\text { EDE } \\
\text { REM }\end{array}$ & $\begin{array}{l}\text { CHA } \\
\text { FATALITY }\end{array}$ & $\begin{array}{l}\text { NCE } \\
\text { GENETIC.E }\end{array}$ \\
\hline $29 \mathrm{E}-0$ & $0.00 E+00$ & $1.42 E+00$ & $7.11 E-04$ & $1.85 E-04$ \\
\hline
\end{tabular}

1 RECEPTOR XNAME (I) $2.29 \mathrm{E}-01 \quad 0.00 \mathrm{E}+00 \quad 1.42 \mathrm{E}+00 \quad 7.11 \mathrm{E}-04 \quad 1.85 \mathrm{E}-04$

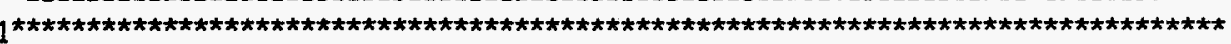
MODAL STUDY

RISKIND-RW: RADIOACTIVE MATE File: riskind.in

TIME-INTEGRATED( 1 YEARS) DOSE CONSEQUENCE (REM)

RECEPTOR 1 : RECEPTOR XNAME (I)

DOWN WIND DIST $(K M)=1.30 E-01$

CROSS WIND DIST $(K M)=0.00 E+00$

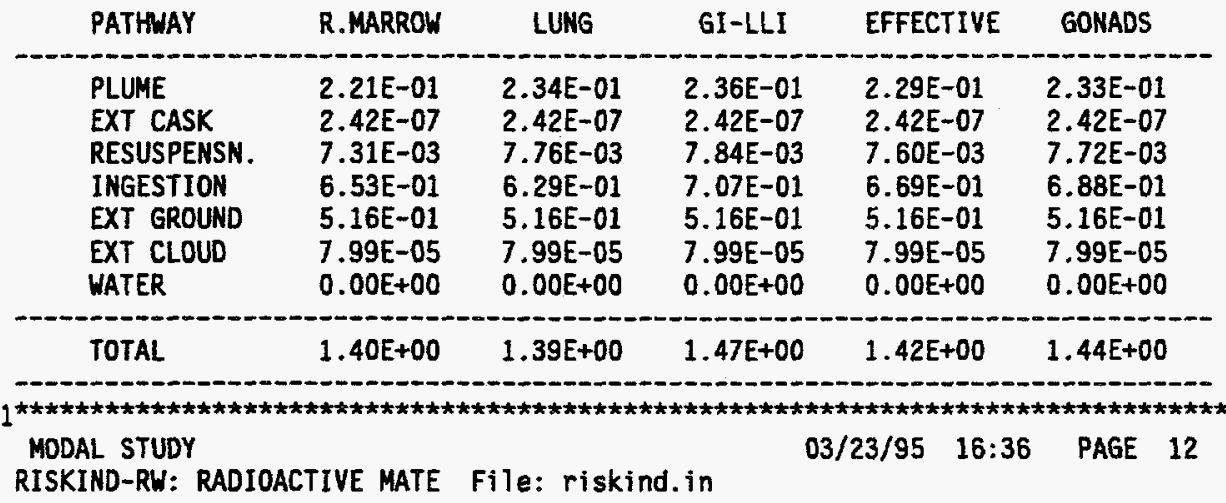

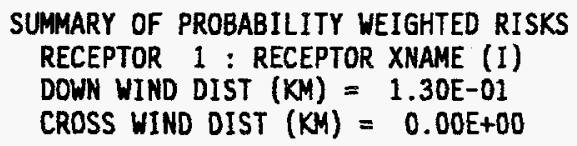

\begin{tabular}{|c|c|c|c|c|}
\hline & \multicolumn{2}{|c|}{ INCIDENT FREE } & \multicolumn{2}{|c|}{ ACCIDENTS } \\
\hline & $\begin{array}{l}\text { TRANSIT } \\
1 / \text { CASK }\end{array}$ & $\begin{array}{l}\text { STOP } \\
1 / \text { STOP }\end{array}$ & $\begin{array}{c}\text { AIR/GROUND } \\
\text { 1/EVENT }\end{array}$ & $\begin{array}{l}\text { WATER } \\
\text { 1/EVENT }\end{array}$ \\
\hline $\begin{array}{l}\text { EFFECTIVE DOSE } \\
\text { (REM) }\end{array}$ & $0.00 \mathrm{E}+00$ & $0.00 \mathrm{E}+00$ & $1.42 \mathrm{E}+00$ & $0.00 E+00$ \\
\hline $\begin{array}{l}\text { LATENT CANCER } \\
\text { MORTALITY } \\
\text { (CHANCE) }\end{array}$ & $0.00 E+00$ & $0.00 E+00$ & $7.11 E-04$ & $0.00 E+00$ \\
\hline $\begin{array}{l}\text { GENETIC EFFECT } \\
\text { (CHANCE) } \\
\text { ACUTE MORTALITY } \\
\text { (CHANCE) }\end{array}$ & $0.00 \mathrm{E}+00$ & $0.00 E+00$ & $\begin{array}{l}1.85 E-04 \\
0.00 E+00\end{array}$ & $0.00 E+00$ \\
\hline
\end{tabular}

